Portland State University

PDXScholar

6-4-1997

\title{
Ethnic and Language Identity Among a Select Group of Vietnamese-Americans in Portland Oregon
}

Jay Owen Peterson

Portland State University

Follow this and additional works at: https://pdxscholar.library.pdx.edu/open_access_etds

Part of the Bilingual, Multilingual, and Multicultural Education Commons Let us know how access to this document benefits you.

\section{Recommended Citation}

Peterson, Jay Owen, "Ethnic and Language Identity Among a Select Group of Vietnamese-Americans in Portland Oregon" (1997). Dissertations and Theses. Paper 5306.

https://doi.org/10.15760/etd.7179

This Thesis is brought to you for free and open access. It has been accepted for inclusion in Dissertations and Theses by an authorized administrator of PDXScholar. Please contact us if we can make this document more accessible: pdxscholar@pdx.edu. 


\section{THESIS APPROVAL}

The abstract and thesis of Jay Owen Peterson for the Master of Arts in TESOL were presented June 4, 1997, and accepted by the thesis committee and the department.

COMMITTEE APPROVALS

Kimberley Brown, Chair

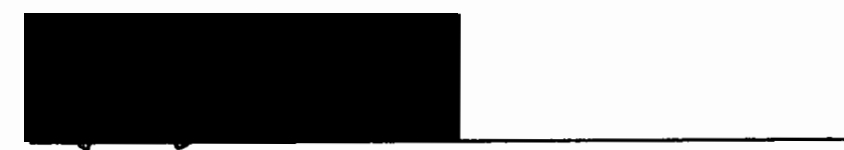

G. Tucker Childs

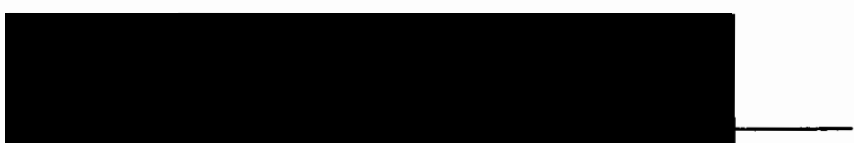

Dalton Miller-Jones
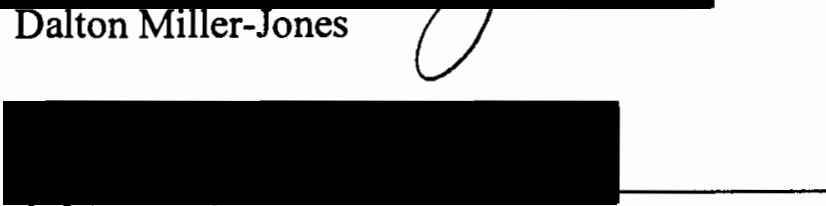

Lêslie Good,

Representative of the Office of Graduate Studies

DEPARTMENTAL APPROVAL

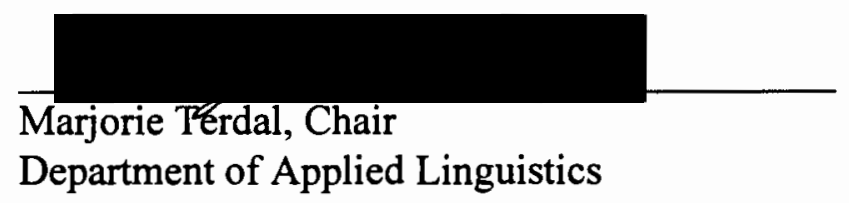

ACCEPTED FOR PORTLAND STATE UNIVERSITY BY THE LIBRARY

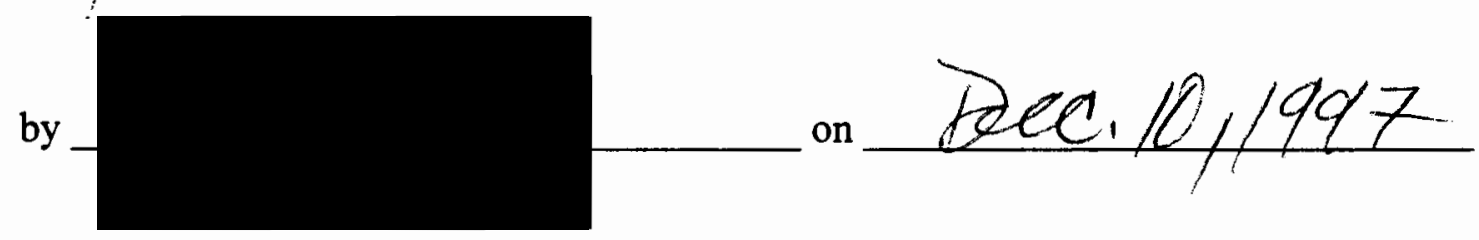




\section{ABSTRACT}

An abstract of the thesis of Jay Owen Peterson for the Master of Arts in TESOL presented June 4, 1997.

Title: Ethnic and Language Identity Among a Select Group of Vietnamese-Americans in Portland Oregon

Vietnamese immigrants within the United States have demonstrated a strong desire to retain their ethnocultural identity. They have also experienced tremendous pressure to use English. What are the implications of these two trends for identity and language choice within the Vietnamese-American community? The presented research used multidimensional scaling (MDS) methods to describe the subjects' ethnic and language identities.

The population for the study consisted of adult Vietnamese-Americans who were born in Vietnam and were currently living in the US. The study had two stages. First, identity labels were generated using a modified Twenty Statements Test. A "Myself" label was added to the list, and the labels were printed on cards in both Vietnamese and English. The labels were sorted by the subjects on the basis of perceived similarity. In addition, subjects chose five cards which were most important for each of the following domains: parents, siblings, friends, school, and work. 
The sorts were submitted to MDS and hierarchical cluster analyses. In addition, the frequencies of labels for each domain were used to explore the effect of context. The group's identity was structured along 3 dimensions: Vietnamese-ness, Chinese-ness, and Americanization vs. being anchored in Vietnam. The most selfsalient items were a sense of shared origin, pride in being Vietnamese, preference for living in the US, family values, a perception of shared physical appearance, common food preferences, and a set of positive personal/group attributes. Items associated with Vietnamese identity (including Vietnamese language) were also very salient. Those items which were heavily loaded on either the Anchored in Vietnam, Americanized, or Chinese vectors were generally less self salient. English language and having a mixed culture were also highly salient. In general the most important aspects of the group's identity were those items which were not heavily loaded on any particular cultural vector. Rather, there was an emphasis on the most socially inclusive items.

The subjects' context specific choices showed relatively little shifting among unique aspects of identity. Instead, there was a stable emphasis on those items associated with a more generally Asian, mixed, and bilingual identity. 


\title{
ETHNIC AND LANGUAGE IDENTITY AMONG A SELECT GROUP OF
}

VIETNAMESE-AMERICANS IN PORTLAND OREGON

\author{
by
}

JAY OWEN PETERSON

A thesis submitted in partial fulfillment of the requirements for the degree of

\section{MASTER OF ARTS \\ in \\ TESOL}

Portland State University

(C)1997 


\section{ACKNOWLEDGMENTS}

I want to thank all those who helped and supported me . In particular, I would like to thank:

my parents, Len and June Peterson; my wife, Lyn Tan; my committee chair and mentor, Kimberley Brown; and my friends, Janet Cowal, Nariyo Kono, Eriko, Maeda, and Masami Nishishiba. 


\section{TABLE OF CONTENTS}

Acknowledgments . . . . . . . . . . . . . . . . ii

List of Tables $\ldots \ldots \ldots \ldots \ldots \ldots \ldots \ldots \ldots \ldots$ vi

List of Figures $\ldots \ldots \ldots \ldots \ldots \ldots \ldots \ldots$ vii

$1.0 \quad$ Introduction $\ldots \ldots \ldots \ldots \ldots \ldots \ldots \ldots \ldots \ldots$

1.1 Statement of the Problem $\ldots \ldots \ldots \ldots \ldots \ldots$

$1.2 \quad$ Research Questions . . . . . . . . . . . . . . . 4

$2.0 \quad$ Review of the Literature $\ldots \ldots \ldots \ldots \ldots \ldots$

2.1 Vietnamese Immigration: Patterns of Acculturation

and Language Use . . . . . . . . . . . . . . 6

$2.2 \quad$ Previous Research on Language and Ethnic Identity . . . . . . . . 12

2.2.1 Traditional Assumptions About Self, Culture, and Language . . . 12

2.2.2 Alternative Contexts and Assumptions About the Relationship

Between Self, Culture, and Language . . . . . . . . . . . . 15

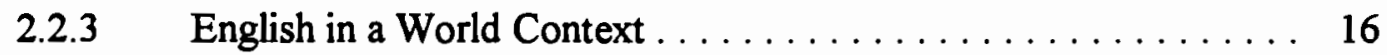

2.3 The Relevance of English's pluricentrism for the United States . . 20

2.4 The Intra-national Context and the Need for a

Psychological Framework . . . . . . . . . . . . . . . . 24

$3.0 \quad$ The Conceptual Framework . . . . . . . . . . . . 27

$3.1 \quad$ The Self, the Processing of Social Information, and Identity . . . . 27

3.1.1 Introduction $\ldots \ldots \ldots \ldots \ldots \ldots \ldots \ldots \ldots \ldots \ldots$

3.1.2 The Role of the Self as a Psychological Construct . . . . . . . . 29 
3.1.3 Information Processing and Schemata . . . . . . . . . . 33

3.1.4 Memory, Schemata, and the Self .............. 35

3.2 Identity Development: Levels of Identity . . . . . . . . . . 39

$3.3 \quad$ Ethnic Identity $\ldots \ldots \ldots \ldots \ldots \ldots \ldots \ldots \ldots \ldots \ldots \ldots$

$3.4 \quad$ Ethnic Identity and Language $\ldots \ldots \ldots \ldots \ldots \ldots \ldots \ldots$

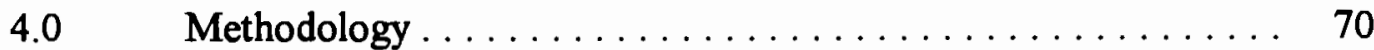

4.1 Summary of Conceptualization and Procedures ......... 70

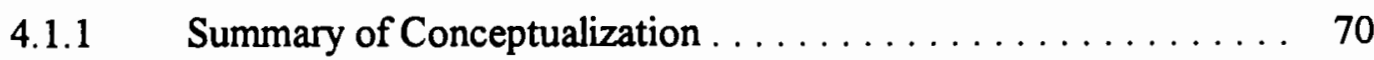

4.1.2 General description of subjects $\ldots \ldots \ldots \ldots \ldots \ldots \ldots \ldots, 72$

4.1.3 Summary of Procedures $\ldots \ldots \ldots \ldots \ldots \ldots \ldots \ldots \ldots$

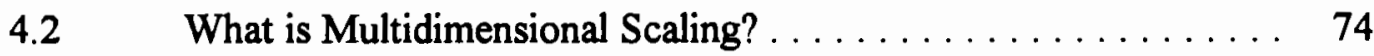

$4.3 \quad$ Cluster Analysis . . . . . . . . . . . . . . . . . . . . 89

4.4 Application of MDS to the Question of Vietnamese Ethnic and

Language Identities $\ldots \ldots \ldots \ldots \ldots \ldots \ldots \ldots \ldots \ldots \ldots$

4.4.1 Identification of All Relevant Objects to be Evaluated. . . . . . . 95

4.4.2 Selection of an Attribute-based or an Attribute -free Approach. . 105

4.4.3 Deciding on the Appropriate Scaling Technique. . . . . . . . . 109

4.4.4 Interpretation of the Results. . . . . . . . . . . . . 113

4.5 Summary of Methodology $\ldots \ldots \ldots \ldots \ldots \ldots \ldots \ldots \ldots \ldots$

4.6 Why Multidimensional Scaling is Appropriate for the Study of

Ethnic and Language Identities . . . . . . . . . . . . 115

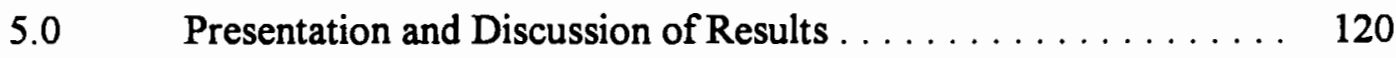




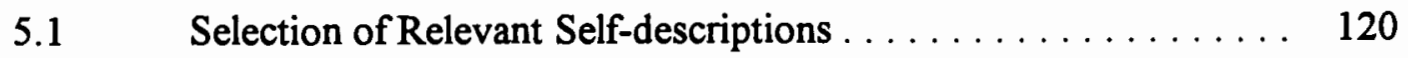

$5.2 \quad$ Results of Card sorting procedure $\ldots \ldots \ldots \ldots \ldots \ldots \ldots \ldots$

$5.21 \quad$ General description $\ldots \ldots \ldots \ldots \ldots \ldots \ldots \ldots \ldots \ldots \ldots \ldots$

5.2.2 Mapping the subject's Perceived Social Space .......... 130

5.2.3 Mapping the Subject's Self-salience Space . . . . . . . . 151

$5.3 \quad$ Context Specific Self-Salience $\ldots \ldots \ldots \ldots \ldots \ldots \ldots \ldots \ldots$

$5.4 \quad$ General Discussion and Implications . . . . . . . . . . . 172

6.0 Limitations and Future Directions for Research .......... 181

Notes............................ 187

References Cited . . . . . . . . . . . . . . . . . . . . . . . . 195

Appendices . . . . . . . . . . . . . . . . . . . . 213

A Informed Consent Forms . . . . . . . . . . . . 213

B Instruction Guides, Instruments, and Demographics Forms for

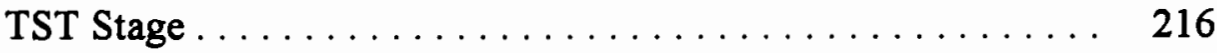

C Data Collection and Demographics Forms For Card Sort Stage . . 226

D Similarity Matrices . . . . . . . . . . . . . . . . . . 233 


\section{LIST OF TABLES}

I Matrix of relationships between ethnic identity and language

identification

II Four categories of self construal related to ethnic and

language identity .

III Potential identity construals for Vietnamese Americans . . . . . . 71

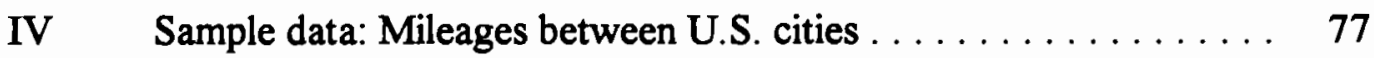

V Stress and improvement of stress by number of dimensions . . . . 83

VII Categorized TST results ..................... 121

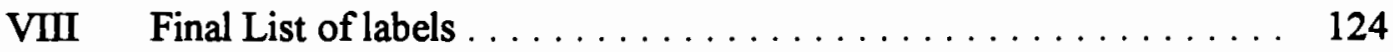

IX Stress and RSQ statistics for social map . . . . . . . . . . 131

X Agglomeration schedule for clustering of social space labels ..... 132

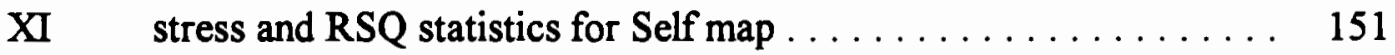

XII Agglomeration schedule for clustering of self space labels . . . . . 153

XIII Ranked distances from Myself $\ldots \ldots \ldots \ldots \ldots \ldots \ldots \ldots 161$

XIV Label frequencies for parent context .............. 168

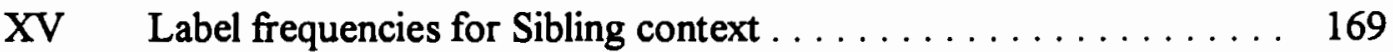

XVI Label frequencies for friendship context . . . . . . . . . . . . . 169

XVII Label frequencies for school context ................ 169

XVIII Label frequencies for work context ............... 170

XIX Frequencies of labels across all contexts . . . . . . . . . 170 


\section{LIST OF FIGURES}

$1 \quad$ Kachru's concentric circles model of English . . . . . . . . . 17

$2 \quad$ Kihlstrom et. al's conceptualization of self as a node within memory 37

3 Markus and Sentis' conceptualization of self as a space

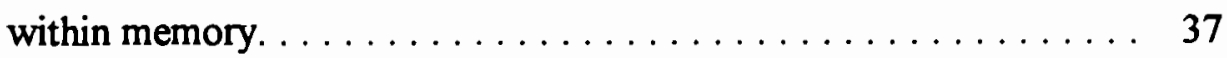

4 Levels of abstraction in identity development $\ldots \ldots \ldots \ldots \ldots, 40$

$5 \quad$ Monism and transference. $\ldots \ldots \ldots \ldots \ldots \ldots \ldots \ldots \ldots$

$6 \quad$ Bifurcation and integration $\ldots \ldots \ldots \ldots \ldots \ldots \ldots \ldots \ldots$

$7 \quad$ Schema structure of monism $\ldots \ldots \ldots \ldots \ldots \ldots \ldots \ldots$

$8 \quad$ Schema structure of bifurcation $\ldots \ldots \ldots \ldots \ldots \ldots \ldots \ldots 6$

9 Schema structure of transference . . . . . . . . . . . . . . . 67

10 One dimensional MDS map of city distances $\ldots \ldots \ldots \ldots \ldots .78$

11 Two dimensional MDS map of city distances .......... 78

12 Scatter plot of disparities for the two dimensional map of

city distances $\ldots \ldots \ldots \ldots \ldots \ldots \ldots \ldots \ldots \ldots \ldots \ldots \ldots$

13 Scatter plot of disparities for the two dimensional map of

city distances $\ldots \ldots \ldots \ldots \ldots \ldots \ldots \ldots \ldots \ldots \ldots \ldots \ldots \ldots$

14 Graph of stress versus the number of dimensions in city

distances solutions $\ldots \ldots \ldots \ldots \ldots \ldots \ldots \ldots \ldots \ldots, \quad 83$

15 Sample dendogram: Results of hierarchical cluster analysis of

beer data . . . . . . . . . . . . . . . . . . . . . . . . . 91

16 Sample MDS map: The concept of "a good teacher" ........ 92 
17 Two dimensional map of city data: Ordinal level scaling . . . . . 111

18 Scatter plot of disparities for ordinal level two dimensional map . . 111

19 Social map scatter plot of stress and number of dimensions . . . . . 131

20 Dendogram of social map labels $\ldots \ldots \ldots \ldots \ldots \ldots \ldots \ldots, 133$

21 Perceived social world (spatial dimensions 1 and 2) $\ldots \ldots \ldots, 135$

22 Perceived social world (spatial dimensions 1 and 3) . . . . . 136

23 Self map scatter plot of stress and number of dimensions . . . . . . 152

24 Dendogram of self map labels . . . . . . . . . . . . . . . 154

25 Self-salience space $\ldots \ldots \ldots \ldots \ldots \ldots \ldots \ldots \ldots \ldots \ldots$

26 Dendogram of distances from "Myself" $\ldots \ldots \ldots \ldots \ldots \ldots \ldots, 160$

27 Self-salience space divided into core self/peripheral self . . . . . . 162

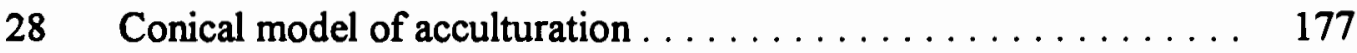




\subsection{Introduction}

\subsection{Statement of the Problem}

As a community, Vietnamese immigrants within the United States have demonstrated a strong desire to retain their ethno-cultural identity (Celano \& Tyler, 1990). ${ }^{1}$ On the other hand, the Vietnamese-American community ${ }^{2}$ has also been under tremendous pressure to acquire and use English (Tollefson, 1989; Wolfram, Christian, \& Hatfield, 1986). What are the implications of these two trends for issues of identity and language choice within the Vietnamese-American community? To what degree, and how, are the identity and language issues interrelated? Is the acquisition of English just one element in the loss of Vietnamese identity during the process of acculturation? Are the two trends indicative of separate and compatible parts of bilingual and bicultural identity development? Is English proficiency an integral part of the maintenance of Vietnamese identity as the community adapts to the new context of the United States? What is the content and structure of perceived language and identity options for the Vietnamese-American community? Further, how should the situation be conceptualized, studied and described?

Most of the theoretical frameworks for assessing the interaction between identity, language choice, and language learning assume that specific languages are specially connected to, or expressive of, specific ethno-cultural identities (see section 2.0). Given this assumption, three possibilities are foregrounded within theory and research for the Vietnamese-American community: (a) increasing use of English could result in the attrition of Vietnamese language along with a diminishing sense of 
Vietnamese identity; (b) the maintenance of Vietnamese identity could result in the non-acquisition of English; or (c) the support of both trends could result in the establishment of bifurcated bilingual / bicultural individuals (e.g., Agar, 1991; Northover, 1988; Weinreich, 1988).

This framework of choices, however, assumes that specific languages are inherently constrained by particular cultures. Recently, this assumption has been questioned for a number of languages, including English (e.g., Clyne, 1992; B. Kachru, 1990). When one considers the sociolinguistic realities of the worldwide spread of English, the assumption of cultural constraint seems particularly inappropriate. The field of World Englishes provides numerous examples in which English has become the medium of an ever widening range of cultural identities. Once the VietnameseAmerican situation is placed within the context of the world wide pluricentricity of English (Clyne, 1990), ${ }^{3}$ another possibility emerges for the Vietnamese community within the United States. Just as English is being used to express an increasing range of cultural identities internationally, Vietnamese-Americans could also choose to maintain their Vietnamese identity within English. Whether they actually do so is an empirical question. Why they may, or may not, express their Vietnamese identity in English involves a number of psychological, social, and political issues. However, considering any of these questions requires the use of a more flexible conceptualization and operationalization of the language and identity issues than has been traditionally used. 
Given this background, the present patterns of Vietnamese acculturation and language use raise the following questions. Do some Vietnamese-Americans feel that their own Vietnamese identity is associated with English; or is English experienced as alien to Vietnamese identity? Are the underlying identity structures of some Vietnamese-Americans consistent with a "nativization strategy", or are the underlying identity structures more consistent with the two languages and cultures being experienced as dichotomous language-culture systems?

The answers to these questions are more than theoretical. If VietnameseAmericans do not associate their Vietnamese identity with English use, then their Vietnamese identity may depend on their use of Vietnamese. On the other hand, if younger Vietnamese-Americans do not consider their Vietnamese identity as incompatible with the use of English, then the choice to use English would not necessarily indicate a shift or loss of identity. However, if Vietnamese identity is not incompatible with the use of English, this could suggest that Vietnamese English may possess unique formal characteristics which should not be confused with low proficiency or fossilized interlanguage. In the first case, the practical issue would become how Vietnamese use (most probably Vietnamese/English bilingualism) can be maintained in the face of intranational English hegemony (B. Kachru, 1990; Tollefson, 1989, 1991). In the second case, as with African- American vernacular English and Chicano English, issues of definition and recognition would be highlighted. Raising 
these issues has both important theoretical and practical implications for researchers, educators, policy makers, community leaders, and individual language users.

The present research was an attempt to examine these issues within an exploratory psychological framework. I used elicited self-descriptions and multidimensional scaling procedures to describe and assess the underlying identity structures of one group of Vietnamese-Americans. In particular, I focused on describing the relationship between the subjects' ethno-cultural and language identities with special attention to the role of English.

\subsection{Research Questions}

RQ1: What items were relevant to the subjects' ethnic identities? Are any languages relevant to the subjects' ethnic identities? If so, which languages are relevant?

RQ2: How did subjects categorize those things which they described as important to their ethnic and linguistic identities? What were the basic identity categories for the subjects? Did they use primarily ethnic, racial, national, or language categories; or did they use some other manner of organization? What attributes, behaviors, languages, values, etc., were included in the different categories?

RQ3: What were the criteria that subjects used to structure their ethnic and linguistic identity choices?

RQ4: How were the languages indicated by the subjects (if any) related to the various ethnic identities involved? Did subjects uniquely associate particular languages 
with particular ethnic identities, or did the languages show mixed associations with a number of ethnic identities? In particular, how were the subjects' Englishspeaking identities related to their various ethnic identity options? How did English compare with the other language options available?

RQ5: How important were the various ethnic and linguistic identities in relation to each other? In particular, how self-salient were the subjects' English-speaking identities in relation to the other ethnic and language choices involved?

RQ6: Which domains were important to the subjects' ethnic and language identities? How did an awareness of these contexts affect the subjects' conceptualization of their identity options? Did some identities or aspects of identity become more or less salient when different domains were considered? 


\subsection{Review of the Literature}

\subsection{Vietnamese Immigration: Patterns of Acculturation and Language Use}

After 1975, southeast Asian immigration into the United States increased dramatically. Events that contributed to this increase included the surrender of South Vietnam, the beginning of civil war in Cambodia, and the end of the monarchy in Laos (Krupinski \& Burrows as cited in Carlson \& Rosser-Hogan 1993, p. 223). The groups involved in this migration included Laotians, Cambodians, Hmong, Vietnamese and ethnic Chinese from those countries. Several acculturation studies of Southeast Asian groups have suggested that Vietnamese immigrants in particular have shown a strong desire to maintain ethno-cultural identity and strong resistance to assimilation. WongRieger and Quintana (1987), for example, found that "most of their subjects could be classified as either separatists or biculturalists" (p. 375). In general, the authors concluded that subjects exhibited a separatist tendency identifying "more highly with their own ethnic group and less with Anglo-American culture . . " (p. 375). In another study of Southeast Asian immigrants (mainly Vietnamese), Celano and Tyler (1990) examined the relationship between a number of variables and acculturation and found that time in country and acculturation were inversely related even when influences of other variables were considered. Celano and Tyler found that subjects "gradually increased adoption of American cultural patterns during the first six months .... [However,] a conflict or turning point appear[ed] to have taken place at six months, followed by a trend toward re-affirmation of Vietnamese culture ..." (Celano \& Tyler 1990, p. 383). The researchers commented that in the case of many southeast 
Asians, especially Vietnamese, the trends of acculturation during the first months of immigration seemed to have been the result of a period of dependence and adjustment. After this adjustment period, employment and increasing functional knowledge of American society eventually led to greater independence and increased contact with other Vietnamese. The fact that there was a strong desire to seek out other members of the community was demonstrated in the waves of secondary migration within the United States in the 70's and 80 's as individuals moved to be near family members, friends, and other Vietnamese in general. This reassertion of community allowed individuals to re-establish many cultural activities and values. ${ }^{4}$ Celano and Tyler (1990) rightly pointed out that it is not clear whether the results of their study are evidence for the permanent establishment of a stable community, or part of a long term (maybe generational) "Ping-Pong" pattern of acculturation. Which explanation prevails will only be discovered after continued research. However, the present trends are testament to the current community's strong desire to maintain native identity.

At the same time that there has been a strong desire to maintain indigenous identity, several researchers (e.g., Tollefson, 1989; Wolfram et al., 1986) have argued that the Vietnamese community has been under tremendous pressure to acquire and use English. This is especially true "among the younger groups, who are motivated to succeed in the educational system and in their careers" (Wolfram et al., 1986, p. 53). In their study of Vietnamese in northern Virginia, Wolfram et al. (1986) suggested that the strong educational and social values orientation of the Vietnamese community not 
only provided a motivational factor for learning English, it also helped determine the acquisitional model. The authors commented that unlike the findings from many other immigrant groups, the findings for the Vietnamese community in Virginia showed little evidence that the community had acquired features from other nonstandard varieties of English. Instead the community seemed to have chosen mainstream standard English as its acquisitional target. This choice may have been influenced by several factors. According to the authors, when compared with other Vietnamese communities (in Houston or Los Angeles, for example) many families in their study had a relatively high social economic status within Vietnam and desired to either maintain or increase that status now that they were in the United States. The authors also noted that the choice of standard English seemed to have been influenced by the community's strong educational values--something which was consistent with both the class aspirations of the subjects and the Confucian background of the culture generally. Therefore, standard English was probably the choice most consistent with the community's values. The choice of standard English as an acquisitional target was important because, according to the authors, the acquisitional target influences subsequent features of an immigrant community's interlanguage.

Describing the formal characteristics of Vietnamese English (VE) interlanguage was another goal of the study. As the authors described, the VE of their subjects did possess a number of unique characteristics:

There is phonological variation including final obstruent devoicing (/fut/ for food), consonant cluster reduction in both initial and final positions 
(/go/ for grow and /tos/ for toast), stopping of interdental fricatives (/doz/for those), among other consonant and vowel modifications (Christian et al. 1983: chap. 5). Morphosyntactically, we find an absence of plural, possessive and third person singular agreement suffixes, copula and auxiliary absence, and inversion in indirect questions (I wonder, where did they go) (Christian et al. 1983: chap. 5). There is also a significant incidence of unmarked tense, as described in Wolfram (1985) (as in I don't have biology this year, I have it last year). (Wolfram et al., 1986, p. 53-54)

Most of these features were consistent with the linguistic background of the subjects and were explainable with traditional models of second language acquisition (Wolfram et al., 1986). As was expected, these items of interference showed decreased rates of occurrence in groups that had either earlier or longer exposure to English and the authors stated that they couldn't be sure, but that they didn't expect any of these features to persist for subsequent generations. However, certain characteristics of Vietnamese English which were also consistent with the linguistic background of the subjects showed a persistence which would not be expected based on the acquisition process alone (Wolfram et al., 1986). These features were identified as those elements that would most likely stabilize as "ethnic markers." The two features given the most discussion by the authors were the absence of final stops in contexts where they would normally be present for standard English speakers (e.g., fast, bad, missed, looked) and a wide ranging use of unmarked tense.

The situation of the Vietnamese in Virginia contained several different trends. First, Wolfram et al. commented that there was a strong sense of community, that the community had a strong desire to maintain Vietnamese cultural identity, and that this 
concern also extended to language issues. The authors argued that the qualitative data suggested there was a generally positive attitude toward (and conditions for) the maintenance of Vietnamese. ${ }^{5}$ Second, and in contrast to the first trend, there was also a tremendous amount of pressure for the community to use English. Third, the authors found that to a large degree Vietnamese and English seemed to be filling different functional roles in several clearly defined cultural contexts (Wolfram et al., 1986). This role specificity could resolve the tension of the first two trends by leading to bicultural bilingualism. Lastly, many subjects also expressed a belief that new generations must somehow blend the best of both cultural influences (Wolfram et al., 1986, p. 49) and this blending was also evident on a linguistic level in the identification of several distinct elements of Vietnamese English. However, in spite of these different trends, the authors generally predicted (a) that English would eventually become dominant (b) that VE was developing toward standard English and (c) that this was one part of the process of acculturation which was in line with the community's social and educational values.

Even though this study presented probably the most complete and detailed account to date of English within the Vietnamese-American community, several limitations are important to note. First, the population for the study included subjects from a broad range of demographic categories, most notably, time in country and age. While some of the subjects were relatively young and had come to the US before adolescence, most of the subjects were adults who had spent the majority of their lives 
in Vietnam. Further, many subjects had been in the US less than 5 years. These demographics undoubtedly affected the results.

Second, many of the subjects in the community which the authors studied were of a relatively high socioeconomic status and tended to have had a lot of language training in formal educational contexts. The authors readily admit that these factors probably worked to reinforce a willingness of the subjects to accommodate to American culture and to choose mainstream standard English as an acquisitional model. Subjects from lower socioeconomic contexts with little formal language education may not have held standard school English as their main acquisitional target and may have exhibited more extensive and persistent areas of variation.

Third, the study looked only at phonological and morphosyntactic features which occurred during a survey interview. The authors did not address the large areas of VE pragmatics or discourse patterns--especially when English is spoken in naturalistic settings with other Vietnamese or other SE Asian immigrants.

Fourth, Wolfram et al.'s discussion of variant features is decidedly within a tradition of structural sociolinguistics. The main concern was identifying those formal features which showed a persistent stability of occurrence and deviation from mainstream standard English. ${ }^{6}$ These were features which, in the authors' words, were probably "communally fossilizing." Features of VE which seemed to be fossilizing were simply labeled as "ethnic markers." However, virtually nothing was said about 
what these features meant functionally or psychologically within the cultural and communicative lives of speakers of VE.

Though this study is one of the few (and perhaps the best) that have attempted to address these issues within the Vietnamese-American community, it also highlights the need for continued research on the cultural and linguistic patterns of VietnameseAmericans and the need for caution in generalizing from the study's conclusions. The situation presented a complicated pattern of social, economic, cultural, and linguistic influences and it is difficult to make say with certainty what will happen in the future or why. With these limitations in mind, one can see that there is much more to know about the connection between language and identity within the Vietnamese-American community. Further, the above example subtly suggests that what is revealed may in part be influenced by the questions that are asked and the general framework of expectations which is used.

\subsection{Previous Research on Language and Ethnic Identity}

\subsubsection{Traditional Assumptions about Self, Culture, and Language}

In the study of the connection between language and identity, many researchers have assumed one or more of the following:

1. Within primary socialization, one cultural context tends to dominate and therefore ethno-cultural identity naturally tends to have a single dominant cultural focus. Other weaker and secondary identifications may also be added, though doing so can often 
present psychological dissonance and conflict (Agar, 1991; Northover, 1988;

Weinreich, 1988).

2. There is a strong connection between particular languages and particular ethnocultural identities (e.g., Guiora, Brannon, \& Dull, 1972; Guiora 1981; Heller, 1987; Ralston, Cunnif, \& Gusttafson, 1995; Robinson, 1991; Shen, 1989).

3. To learn a language other than the individual's "native," or "primary" one introduces areas of potential cultural and social interference, confusion, and conflict; therefore, learning another language necessarily involves a variety of problems, tensions, adjustments, and changes in the learner's identity (Berry, 1986; Weinreich, 1988; Northover, 1988; Guiora, et al., 1972; Guiora, 1981; Shen, 1989).

For reasons which will become apparent, I will refer to these collectively as assumptions of "linguicultural monism." They constitute a vision of culture as a unitary system which is intimately connected to a specific language. 'The assumptions of monism are common for the good reason that there are many groups for which these assumptions do seem descriptively appropriate:

Mainland Chinese (Rin, 1982; Shen, 1989)

Hong Kong Chinese (Pierson, Fu, \& Lee, 1980)

French Canadians (Gardner \& Lambert, 1972; Smolicz \& Secombe, 1985)

Japanese (Baxter, 1980; Doi, 1973; Suzuki, 1978; Wierzbicka, 1991)

Malays (Asmah, 1975; Smolicz 1981a \& 1981b)

Poles (Smolicz 1981a; Smolicz \& Secombe 1985)

Baltics (Smolicz \& Secombe, 1985)

Greeks (Smolicz \& Secombe, 1985)

Catalans (Conversi, 1990) 
Probably the most vivid examples of the above attitudes, or assumptions, are given in the following statements which Hong Kong Chinese students in Pierson, Fu and Lee's (1980) study most strongly identified:

"If I use English it means that I am not patriotic."

"At times I feel that by using English I will become like a foreigner." "When using English, I do not feel that I am Chinese any more."

The real sense of anomie that such a group can feel when sensing the loss of its mother tongue is poignantly expressed by the Catalan anthropologist Oriol Pi I Sunyer:

[This is] . . the most important cultural question and political consideration with which this generation of Catalans must struggle: is it possible to be Catalan without speaking the language? If the answer is in the affirmative, what does it mean to be Catalan once the language takes a secondary position as a symbol of identity?

(as cited in Conversi, 1990, p. 56)

The Cuban writer Carlos Montaner expresses similar feelings when he exclaims that "we [i.e., Cubans] cannot do without our own tongue without brutally mutilating our individual consciousness, without being left without blood" (Montaner, 1992, p. 164).

Even though the above attitudes can be associated with a strong ethnolinguistic vitality (Giles, Bourhis, \& Taylor, 1977), one should resist the tendency to uncritically romanticize them. They are the result of specific social, historical, and ideological pressures, which are not always positive in source or effect (Smolicz, 1986, 1992). As Hoffman (1991) has argued, one origin of the belief that specific languages are specially connected to specific ethnic or cultural identities can be found in Romantic Nationalism. "The philosophical underpinnings of this attitude was the ideal of one nation, one language" (Hoffman, 1991, p. 157). On a social group level, these beliefs 
produce a strong normative ideology for what constitutes both the culture's linguistic boundaries and the language's cultural boundaries. The result of this ideology has been that many countries choose to "ignore the language diversity within their frontiers" (Hoffman, 1991, p. 57), choose "a history of suppression of minority languages" (Hoffman, 1991, p. 57), and promote societal monolingualism of elite mother tongues. On an individual level, these ideological ideals often result in the identity ideal of monocultural monolingualism in which non-mother tongue learning tends to be experienced as varying forms of personal dissonance and alienation (Ree, 1980).

Neither should one naturalize linguicultural monism by uncritically generalizing it. In many cases, the connection between language and cultural identity seems much looser, multifarious, porous, and negotiable. It is in these examples that interested researchers have begun to expand the present state of questioning.

\subsubsection{Alternative Contexts and Assumptions about the Relationship Between Self, Culture, and Language}

After a number of quantitative, ethnographic, and diary studies of immigrant attitudes and values in Australia and South East Asia, Smolicz (1980, 1981a \& b, 1985 ) identified several groups for which language does not seem to be "a core cultural value":

Many Jews outside of Israel (Smolicz, 1981a; 1985)

Dutch in Australia--possibly elsewhere (Smolicz, 1981b)

Chinese in much of SE Asia (Smolicz, 1981a \& 1981b)

Several groups within India (B. Kachru, 1979b; Kubchandani, 1983;

Pandit as cited in Pride, 1983; Pride, 1983). 
Language is not a core anchor of ethnic group identity in any of the above examples. Similarly, Giles, Taylor, Lambert and Albert (1975) have identified a group of young non-French speaking Franco-Americans for whom language appears to have very little salience as an anchor of ethnic identity. Pradell de Latour (1983) has presented similar examples of French ethnics in the Lorraine coal basin for whom "cultural practices are void of any identification references of a francophile nature" (p. 87). He has offered the following example as typical of the feelings of many French in the region: "My great grandfather was born in 1866 . He didn't speak a single word of French and yet he never diverged from his belief until his death in 1950. He would say 'Ich bin Franzose"' (as cited in Pradell de Latour, 1983, p. 87).

Collectively, these examples suggest that although many people may feel a close connection between specific ethno-cultural identities and specific languages, "there should be no assumption that ethnic and linguistic identities [must] overlap" (Khan, et al., 1983).

\subsubsection{English in a World Context}

The same sociolinguistic realities are also present within the English-speaking community. Around the world, the number of English speakers has been increasing at a rate which is unprecedented in the linguistic history of any language (B. Kachru, 1994). Because of this spread, traditional beliefs that the cultural and communicative models for acquisition are "native" (monolingual / monocultural) speakers from Britain, 
the US, Australia, and New Zealand have largely become mythology (B. Kachru, 1994).

B. Kachru (1989) has discussed the changing demographics of English within a framework of three concentric circles. ${ }^{8}$ This framework is presented below as Figure 1. The Inner Circle represents the traditional set of English-speaking countries in which English is a dominant language. The Outer Circle represents those "regions which have passed through extended periods of colonization" (B. Kachru, 1986, p. 87) by Inner

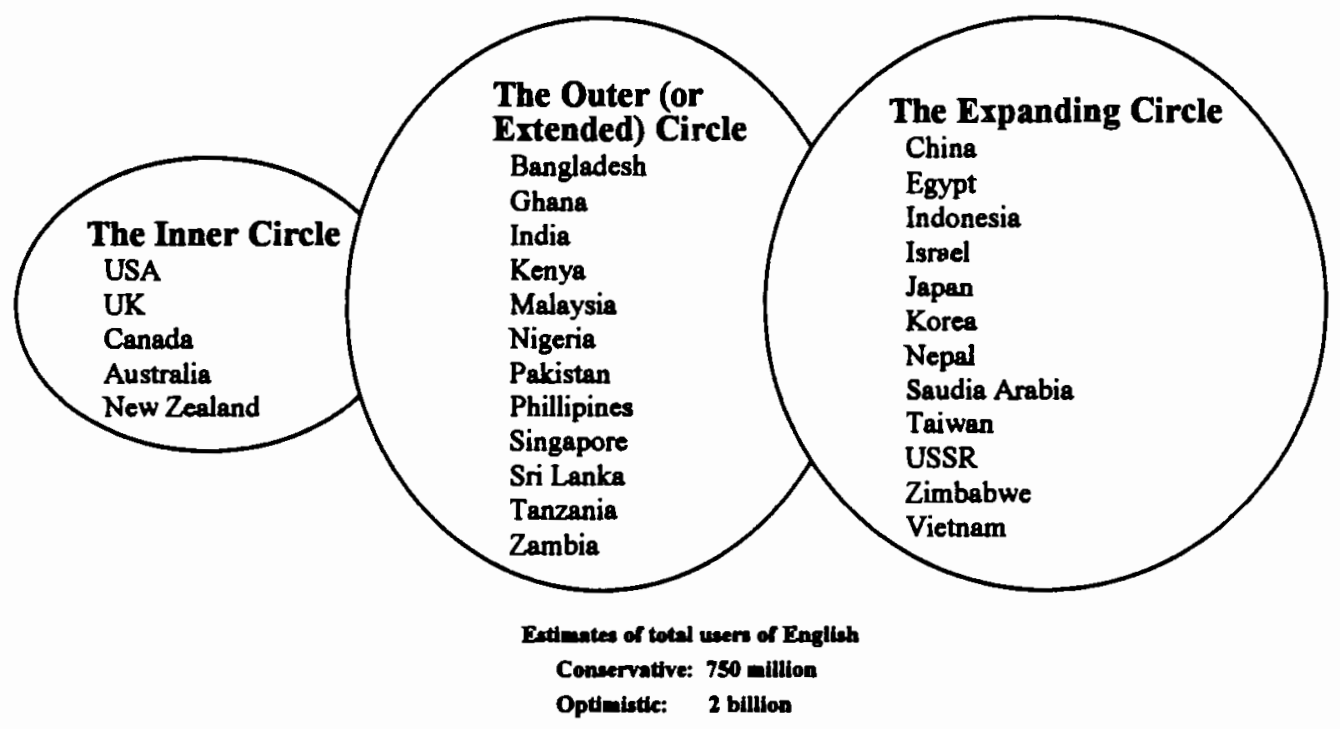

Figure 1. Kachru's concentric circles model of English

Circle countries and as a result have a large number of English-speaking citizens.

Within Outer Circle countries, English has a dominant role in many important intranational domains of use, and many speakers in the country use English as a major language of daily activity. The Expanding Circle represents those countries in which English has an important role for international purposes (e.g., international business, 
science) though its role intranationally is limited. Consequently, the Inner Circle countries have often been referred to as "native speaking" countries, Outer Circle countries have been called "non-native," or English as a second language (ESL) countries, and Expanding Circle countries have been designated as English as a foreign language (EFL) countries. ${ }^{9}$

According to even the most conservative estimates, the number of speakers in the Outer Circle is quickly surpassing the number in the Inner Circle, and the number in the Outer and Expanding Circle combined easily exceeds the Inner Circle by estimates of two to one (B. Kachru, 1994). In a situation in which "there are more Chinese learning English as a foreign language than the total population of the UK [and] . . a conservative estimate of the users of English in India as five percent of the population makes it the third largest English using country after the USA and UK" (B. Kachru, 1994, p. 21), traditional notions of the cultural identity of English must inevitably change.

In large part, the unprecedented spread of English has been the product of colonialism fueled by the technological pressures of development politics (B. Kachru, 1994; Pennycook, 1994). ${ }^{10}$ However, in spite of the pressures of imperialism and modernization, cultural and linguistic influence has not been unidirectional. More often than not, Outer Circle countries have "played that age old trick" (B. Kachru, 1990, p. 12) of co-opting and acculturating the language of the colonizers. Just as the spread of English worked to colonize India, for example, the Indian context also worked to 
acculturate English and make it more Indian in content, form, and use (B. Kachru, 1983; Pandharipande,1992). The result of this "alchemy" (B. Kachru, 1990) within the Outer Circle has been the development of indigenized, or nativized, varieties of English which express an expanding range of ethnic and cultural identities. These "new Englishes" vary from Inner Circle varieties on every level: lexical, phonological, morphological, syntactic, pragmatic, and discoursal (see Lowenburg, 1984 for a concise summary of several examples). There are too many examples to discuss here, though the Englishes of Singapore, Malaysia, India, Nigeria, and the Philippines have been studied extensively (for introductions to the field see Brown, 1993; Cheshire, 1991; B. Kachru, 1982 \& 1992; Noss, 1991; Pride, 1983). As a consequence, English no longer has "one defining context, but many--across cultures and languages" (B. Kachru 1990, p. 13).

For many culturally-diverse groups in the Outer Circle, English is now learned as a primary language of daily use and has become a part of the personal and cultural identities of its users. However, for many of these culturally diverse speakers, English “... is the means of expression of ... [their own] culture and not an imitation of the culture of Great Britain, the United States or any other native speaking country (Smith, 1991, p. 34). ${ }^{11}$

In short, English has become part of these speakers' own ethno-cultural identities. "When we speak of a person's identity we are referring to aspects of the world that one has taken over as their own, both consciously and unconsciously ..." 
(Reminick, 1983, p. 18). It is precisely these aspects of identity and ownership that are so crucial, and so elusive, for establishing the status of new cultural varieties of English.

The statements of many indigenous authors in these countries have been testament to their own feelings of cultural ownership over English. ${ }^{12}$ Among the more well known of these assertions is Chinua Achebe's often quoted statement that “. . the English language will be able to carry the weight of [an individual's] African experience. But it will have to be a new English, still in communion with [his] ancestral homeland" (Achebe, as cited in Smith, 1981, p. 25; my emphasis). ${ }^{13}$ Similarly, Pandit (as cited in Pride, 1983) has commented that English is now "just another Indian language" and as such "removing English from the Indian scene at the moment would be disastrous for the Indian languages and literatures" (as cited in Pride, 1983, p. 34; my emphasis). Santos Castillo (as cited in Llamzon, 1983) and Llamzon (1983) have called English a Filipino language and both Goh (1993) and Kwan-Terry (1991) have discussed English as a southeast Asian language.

Though this summary has only touched the surface of many important issues, I think it is sufficient to suggest that the field of World Englishes continues to provide a myriad of varied examples which support the belief that "when any language becomes international in character it cannot be bound to one culture" (Smith, 1991, p. 34) and that the assumption can no longer be made that the choice to learn or use English--even as a primary or first language--must be linked to any particular ethnic or cultural identity. 


\subsection{The Relevance of English's Pluricentrism for the United States.}

The relevance of English's pluricentricity extends beyond the international setting (Rodby, 1992; Tickoo, 1991). Recognizing the cultural and linguistic diversity of the United States' population, it is reasonable to ask whether similar processes of nativization are taking place for culturally and linguistically diverse groups within the United States and if so, what sort of conceptualization can be used to adequately recognize and describe it.

In spite of the above mentioned developments in sociology, ethno-anthropology, and in the field of World Englishes, many authors and researchers within the Inner Circle have still assumed that English must be somehow specially connected to, or expressive of, a particular ethnic and cultural identity. Acculturation theorists have assumed that language preference in general, and the choice of English in particular, is a reliable indicator of an individual's ethnic and / or cultural identity (e.g., Felix-Ortiz, Newcomb, \& Meyers, 1994; Sayegh and Lasry, 1994). Authors in psychology have argued that language choice among English-speaking bilinguals is guided by the psychological pressure to maximize congruence between linguistic and cultural identities (e.g., Weinreich 1988; Northover, 1988). Researchers in applied linguistics have argued that whether or not one wishes to take on the cultural identity of a language is an important variable in second language learning (e.g., Guiora 1981; Guiora, et al., 1972; Lambert, 1969; Robinson, 1991). Some authorities within language pedagogy have argued that English's cultural identity is under attack from foreign influences (e.g., Pascasio, 1976 as cited in Pride, 1983; Prator, 1968; Quirk, 1987); and a few authors in critical 
linguistics ${ }^{14}$ have argued that speakers of nativized varieties are not culturally authentic or legitimate (e.g., Dasgupta, 1993; Phillipson, 1992). ${ }^{15}$

Previously mentioned developments would suggest that an assumption that specific languages are most naturally connected to, or expressive of, specific identities does not reflect any psychological or linguistic inevitability. Instead, these attitudes are normative in nature and ideological in origin (B. Kachru, 1987, 1991, 1994; Pennycook, 1994). As a result, a more complicated conceptualization of the relationship between language and identity is needed, in particular, one that considers the possibility of nativization and recognizes the ideological nature of linguistic attitudes and theories.

The cultural and linguistic boundaries of English are changing with its internationalization. Similarly, the cultural and linguistic boundaries of the United States are also changing. With these changes have come challenges and re-definitions of what it means to speak English and also what it means culturally and linguistically to be an American. Because of these realities, any valid theoretical framework for the study of English within the US must also." . include the search for an understanding of [English's changing] cultural boundaries and an attempt to come to terms with these boundaries" (Kramsch, 1993, p. 12). Therefore, given the present diversity of the English-speaking population of the United States, it seems reasonable to ask if the same tensions and processes which are influencing English internationally are also present within the United States. If the nativization of English is an empirical reality 
internationally--having functioned to maintain indigenous identity against the pressures of colonization--then it could also be a functional strategy for culturally-different peoples within the United States who are trying to maintain their identity in the face of enormous pressures to assimilate. It is conceptually possible that nativizing English could offer a positive and effective strategy for balancing an individual's internal identity needs and external functional pressures during cultural contact. Whether this strategy is actually used by specific groups within the United States is an empirical matter. Why this strategy is chosen, or not chosen, by specific groups can only be addressed by considering a number of psychological, social, and political issues.

A pluricentric paradigm could also have a positive effect on how VietnameseAmericans themselves see their own language choices--a possible effect more consistent with the ethical principle that social science research should be relevant and useful to the particular group under study (Hamnett, Porter, Signh, \& Kumar, 1984). The assumptions behind a pluricentric paradigm may better meet all of these methodological and ethical criteria. On a more general level, the possibility of Vietnamese-American ownership of English could also suggest that the concept of Englishes (in all its theoretical and ideological implications) is legitimate on both an international and an intranational level. However, none of these issues can be addressed unless traditional assumptions of linguistic and cultural monism are set aside and the issue of identity and language is raised as an empirical question. 


\subsection{The Intranational Context and the Need for a Psychological Framework}

So far, I have attempted to justify a particular question: is English a part of Vietnamese ethnic identity for some Vietnamese within the US? I have also argued that this sort of question has rarely, if ever, been asked. Traditional sociolinguistic studies (e.g., Wolfram et al. 1986) have addressed the issue as primarily a linguistic one--does VE possess unique and persistent characteristics which mark it ethnically? However, the previous discussion suggests--and I have argued elsewhere (Peterson, 1994)--that answering this question necessitates the use of more than just formal linguistic data. Merely establishing that a variety is unique and stable is not sufficient to tell one whether the variety is an interlanguage fossilization or a new English. To answer this question, one must also present evidence that the particular linguistic phenomena are, or are not, salient to a user's ethno-cultural identity.

Within the Outer Circle, the issues of identity and cultural ownership have been addressed in basically two ways. Some authors have presented evidence and arguments that a particular variety of English in a country has become institutionalized--i.e., it is used primarily intranationally across a wide variety of domains (including official, literary, educational, etc.,), has developed its own independent norms, and expresses the particular cultural context of its development (e.g., B. Kachru, 1982, 1990; Lowenberg, 1988; Mohanan, 1992). Other Outer Circle authors have appealed to their political authority to simply assert the right of their own communities to decide for themselves whether they own English and how their use of English should be described 
(e.g., see Prabhu's statements in Pakir, 1993, p. 85). ${ }^{16}$ While these strategies have worked to politically establish the legitimacy of many varieties internationally, they are problematic for use within the Inner Circle. In countries such as the US which are socially and linguistically characterized by minority-majority relations (Conklin \& Lourie, 1983), macro level structures related to institutionalization (e.g., educational and official domains of use, norms for standardization, etc.,) rarely reflect minority influences or attitudes. Further, if these structures do reflect minority influences, they are often described as deviations from an idealized mainstream--whether real or imagined--and are devalued. In these cases, more individual and direct measures of the connection between identity and language are required. As mentioned above, there are a few psychological frameworks that have been developed for the study of concepts such as "language ego" (e.g., Guiora, 1981; Northover, 1988). However, these theories have either explicitly or implicitly assumed that English is culturally bounded (see section 2.2 .1$, p. 12). What is needed, then, is a firmly grounded psychological framework which can also take into account the pluricentricity of English. Because these issues depend on a more detailed understanding of identity than is contained in most linguistic research, it will be necessary to detour into psychology proper. The level of detail in the next section will be necessary for three reasons:

1. A certain background vocabulary is necessary to later address language issues.

2. The conceptualization and vocabulary will clarify potentially ambiguous distinctions. 
3. The concepts involved need to be linked to their operationalization in a complete and psychologically precise manner. 


\subsection{The Conceptual Framework}

\subsection{The Self, the Processing of Social Information, and Identity}

\subsubsection{Introduction}

In many ways the content and structure of this section are guided by several problematic aspects of previous studies relevant to understanding language and ethnic identity. Research in the area of ethnic identity has been carried on in many different fields. Given the range of approaches, the number and variety of different groups studied, and the number of different purposes for the research, the subject as a whole has been characterized by a great deal of fragmentation (Phinney, 1990). First, there has been a tendency for researchers to ignore crucial definitional and conceptual issues. Isajiw (1980), for example, found only thirteen articles out of the sixty-five that he reviewed even attempted a definition of basic concepts. In another review of research on the subject, Weinreich (1988) found that two thirds of seventy empirical studies in refereed journals since 1972 offered no definitions of foundational terms and concepts (In Phinney, 1990). Similarly, Phinney (1990) found that more than one fourth of the juried articles in 36 journals since 1972 offered no definition of ethnic identity. More recently, in a review of empirical studies related to ethnicity from 1970-1993, Leets, Giles, and Clement (1996) found that the vast majority of the articles reviewed offered either no definition, or definitions of highly questionable validity (e.g., geographical residence, birthplace etc.). 
Berger \& Badac (1982) has attributed some of these problems to researchers' generally un-analytic attitude toward the self which has resulted in a propensity for "quick and dirty" conceptualizations that are most focused on the immediate needs of the researcher. A serious result of this attitude has been the general lack of adequate theoretical ground work. In Leets et al.'s (1996) review, for example, $74 \%$ of communications articles and $84 \%$ of social science articles contained no clear conceptual foundation linked to more general theories of identity. Further, Phinney (1990) has suggested that "researchers often appeared unaware of previous work; that is, they did not cite relevant prior work. Therefore, there was much duplication of effort as researchers developed new measures independently" (p. 500). In short, researchers have spent a lot of time working individually with particular ethnic groups and developing approaches and measures with limited generality, limited theoretical foundation, and often questionable validity (Phinney, 1990). Consequently, when one considers the wide range of potential approaches available, it is not often clear why particular authors have decided that a specific approach, method, technique, or instrument was the most appropriate choice for their purposes. In many other cases, it is clear that authors have had a more extensive theoretical background in mind, though this background of information and decisions has rarely appeared overtly in the reporting of the research itself. In an attempt to avoid these shortfalls, I will approach the subject by treating the issues as a narrowing set of topics:

1. The self as the most inclusive construct necessary for understanding all levels of human information processing and identity development. 
2. Information processing as schema development.

3. Identity as the development of various levels of self-schemata.

4. Social group identity as a distinct level of schema development within the process of identity development in general.

5. Ethnic identity as a potential (though not necessary) process of schema development within the more general processes of social identity development.

6. Language identity as one level of social identity development that may, or may not, be associated with any other particular categories of social identity.

At each stage, I will review the relevant research, and attempt to relate this information to appropriate language issues. The end goal (section 3.4) will be the description of a psychological framework for conceptualizing the relationship between language and ethnicity which is theoretically consistent with both the pluricentric assumptions of the proposed question and the methodology described in section 4.0.

\subsubsection{The Role of the Self as a Psychological Construct}

Early theorists on the subject (James, 1890, 1915; Sarbin, 1962) argued that one defining human characteristic is the ability of individuals to move out of the subjectivity of their own experience and regard themselves as objects. Psychologists have found that this ability is important precisely because individuals' concepts of themselves are important variables affecting their perception, sense of agency, and behavior. In short, an individual's self-concept is a central structure involved in much of the individual's cognitive, affective, and behavioral processing (Ghee, 1990; Markus \& Kitayama, 1992; Markus \& Sentis, 1982; Kilstrom, Cantor, Albright, Chew, Klien, 
\& Neidenthal, 1988). These psychologists also argued that the self is not just one of many cognitive structures, but rather the first and central structure through which all experiences are processed. In short, the self should be seen as "the frame of reference of which all other perceptions gain their meaning" (Combs \& Snygg, 1959, p. 42).

Consequently, most psychologists who address issues related to identity have assumed that some sort of central integrating structure within the individual is a logical precondition for unity and consistency within the individual's personal and social experience (Demo, 1992). As Allport (1937) argued, there must be some stable selfstructure that is a mediator of experience or one wouldn't feel that one is the same person across time. Allport's view has gained widespread consensus within psychology. For example, in a broad historical review of psychological views of selfconcept and identity, Markus and Sentis (1982) found overwhelming agreement (behaviorists being the one exception) that the self should be approached as the central point of orientation through which an individual's perception and organization of the world is accomplished. They found that the idea of the self as a distinct and unique central mediator of experience is either assumed or directly implied by most forms of cognitive, social, and clinical psychology; and that within the study of identity and personality the idea is a virtual given. ${ }^{17}$

More recently, this basic assumption has been extended by cultural psychologists who have conceptualized cultural differences as differences within the self (for a review of dominant themes in cultural psychology see Shweder \& Sullivan, 1993 ). On this view, culture is defined as: 
. . . a subset of "mind"; mind (assumed to be latently available and accessible through each individual's nervous system) is conceptualized as an "etic grid", a heterogeneous and inherently complex collection of all possible or available meanings. A culture from this perspective is that subset of possible or available meanings which by virtue of enculturation (informal or formal, implicit or explicit, unintended or intended) has so given shape to the psychological processes of individuals in a society that those meanings have become, for those individuals, indistinguishable from experience itself.

(Shweder \& Sullivan, 1993, p. 512)

Quite simply, this view conceptualizes the construct of self as logically prior to the concept of cultural variation in individual experience and behavior. ${ }^{18}$ Consistent with the assertions in section 2.0 , this view places identity in the forefront and conceptualizes cultural variation as emergent differences in the content and structure of the self.

Empirical research using this framework has supported traditional assumptions that the self is the central point of orientation in experience. However, research in cultural psychology has also suggested that assumptions about the centrality of the self should be distinguished from the status of specific assertions about the nature of self structure (Markus \& Kitayama, 1991, 1992, 1994). Traditional theories related to selfconcept have been based in Western (mainly American) contexts. As a result, these theories have emphasized many findings which may not be universal (e.g., the assumed importance of the fundamental attribution error). More recent research on a wider variety of cultural groups suggests that there are probably a variety of self-construals which vary along a number of dimensions and that these differences "can influence, and in many cases determine, the very nature of individual experience" (Markus \& Kitayama, 1992, p. 3)--including many aspects of cognition, emotion, motivation and 
behavior (for other related collections of research in the area of self-psychology and culture see Berry, Poortinga, Segall, \& Dasen, 1992; Eckensberger, Lonner, \& Poortinga, 1979; Shweder \& Levine, 1984; ; Stigler, Shweder, \& Herdt, 1990). ${ }^{19}$

In a parallel manner, several linguists interested in cultural variation within English (e.g., Kachru, 1994; Khubchandani, 1983; Pennycook, 1994) have argued that the main distinguishing feature between the Inner, Outer, and Expanding Circles is a difference between how language and identity are related for the various groups concerned (see section 2.0). Some of these same authors (Kachru, 1994; Khubchandani, 1983; Pennycook, 1994; Thumboo, 1994) have made the suggestion that not recognizing the identity issues involved has resulted in many traditional concepts being inappropriately extended to cultural contexts in the Outer Circle. ${ }^{20}$

A few authors have even argued that recognizing the sociolinguistic differences between the Inner and Outer Circles suggests that the issues of language and identity are central to the nature of language itself. According to Pennycook (1994), for example, the spread and nativization of English has demonstrated that "anything we might want to call a language is not a pregiven system, but a will to community" ( $p$. 29). In this sense, rather than communication being defined by unified and stable systems of rules (i.e., well defined languages, dialects, etc.,), it is seen as a continuous creative process of identity relations which are, as often as not, characterized by difference, fragmentation, struggle, and paradox (Pennycook, 1994). As Pennycook argues: "Linguistic behavior . . . is better understood in terms of an open series of 'acts of identity' in which people reveal both their personal identity and their search for 
social roles" (Le Page \& Tabouret-Keller as cited in Pennycook, 1994, p. 29). I use Pennycook not so much to pursue or support his particular views about the ontology of language ${ }^{21}$ as to point out that as English is studied in a greater variety of social and cultural contexts, issues of identity are becoming more central to what had previously been studied in a more strictly linguistic manner.

Given the general consensus within psychology on the central place of the self, and the suggestion that differences in self (including differences related to language) may influence fundamental aspects of individual experience and behavior, it is an assumption of this paper that an understanding of the self is ultimately relevant to most (if not all) issues of language choice and use.

\subsubsection{Information Processing and Schemata.}

Since the self is only accessible on a phenomenological level (Berger \& Badac, 1982; Markus \& Sentis, 1982; Wallman, 1983), many psychologists have assumed that the basic processes involved in identity formation and maintenance must be similar to those used in human perception and information processing generally (Fiske \& Taylor, 1991; Kihlstrom \& Cantor, 1984). Therefore, cognitive and social psychologists using an information-processing approach to cognition and behavior, have focused on the general cognitive structures and processes by which individuals organize the world as well as their positions in it. This network of relationships between the world and the self become represented within the individual's memory in the form of schemata. ${ }^{22}$ In order to understand and act with any consistency in the world, "a human being--and 
probably other animals as well--builds up an internal representation, a model of the universe, . . . a cognitive map ..." (Miller, Galanter, \& Pribram as cited in Markus \& Sentis 1982 , p. 43). The basic components of this internal representation are schemata. Schemata are dynamic models or hypotheses with which the individual actively tries to understand the world. They are developed and maintained through both bottom-up and top-down modes of processing (Fiske \& Taylor, 1991). Generally, they are internal conceptual models of different aspects of an individual's experience and are built from perceptual data through the process of abstraction and inference (bottom-up processing). However, schemata also affect the very processes of abstraction and inference that are used to maintain them. That is, schemata act as both cognitive templates for understanding data and also as processing plans for further focusing the individual's perceptions (top-down processing). This interactive process of schema development and maintenance is used both to understand the world and as a guide to act within it. ${ }^{23}$ As such, schemata are used in almost every aspect of an individual's experience, including: (a) identifying common objects (Cooper and Shepard, 1984); (b) perceiving other people (Fiske \& Dyer, 1985; Fiske \& Taylor, 1991; Markus, Smith \& Moreland, 1985; Martin \& Clark 1990); (c) learning language (See Markus \& Wurf, 1987); and (d) participating in social and communication rituals (Abelson, 1981; Berger \& Badac, 1982; Sperber \& Wilson, 1988). In short, "schemata are the central cognitive units in the human information-processing system" (Markus \& Sentis, 1982, p. 43). 


\subsubsection{Memory, Schemata, and the Self}

Within an information-processing approach, memory can be conceptualized as an associative network of schemata (Bower \& Gilligan, 1979; Kihlstrom et. al., 1988) and "the self can be conceptualized as a system of self-schemata" (Markus \& Sentis, 1982, p. 49; my emphasis). "[A self-schemata is a] . . generalization about the self derived from repeated categorizations and evaluations of behavior by one's self and others within a given domain" (Markus \& Sentis, 1982, p. 45).

Very simply, as people gain experience, they begin to make categorizations and judgments about themselves within a variety of domains. Through repeated experiences, individuals build schemata, hypotheses, and expectations about their own probable feelings and behavior within various situations or categories of situations. ${ }^{24}$ That is, in much the same manner as individuals become informal "experts" about others, they also become experts about themselves ${ }^{25}$ (Kihlstrom, et. al., 1988; Markus \& Sentis, 1982; Markus, Smith \& Moreland, 1985; Sarbin, 1962;). ${ }^{26}$

We come to understand that we are shy, or creative or fat, or stubborn, or intimidated by large groups, or pathologically late for appointments, fascicle with numbers, a good cook, a rotten child, or a loving parent. At the same time we come to know a great deal about what it means to be shy, creative, fat, etc. (Markus \& Sentis, 1982, p. 45)

Considering the vast amount of autobiographical information that an individual possesses, the self is probably "one of the richest, most elaborate knowledge structures stored in memory" (Kihlstrom et. al., 1988, p. 150). As a result of this high level of elaboration, the self tends to dominate perceptual and information processing (Kihlstrom et. al., 1988). However, at the same time, considerations of cognitive 
economy which drive schema formation in the first place also suggests that "the self does not contain all the information that is known about oneself" (Kihlstrom et. al., 1988, p. 150). Very simply, not all information about one's self is equally important within memory processing. This selectivity in the content of self-structure is evident in the "self-reference", or "self-latency" effect in memory recall (for summaries of this research, see Fiske \& Taylor, 1991; Kihlstrom et. al., 1988; and Markus \& Sentis, 1982). The work of Rogers, Kuiper, and Kirker (1977; cited in Kihlstrom et. al., 1988) provides an illustrative example of the self-reference effect:

[When subjects were given] . . orthographic, phonemic, and semantic orienting tasks and then asked to judge whether some traits described themselves ... the items presented in self reference condition were better recalled than those presented for the other types of judgments.

(Kihlstrom et. al., 1988, p. 151)

Because the self is selectively structured, at any point, information-processing tends to be done in a way that supports the particular content and associations within existing self-structure (Demo 1992). Through this interplay, persistent patterns in an individual's experience become central organizing features which affect the processing of subsequent experiences. "Self-schemata search for information that is commensurate with them and direct behavior so that it is commensurate and consistent with them" (Markus \& Sentis, 1982, p.45). Eventually, these structures which stand out in an individual's memory "become the self and become the ones most salient, central, and important in organizing information about the self and perhaps the social world in general" (Markus \& Sentis, 1982, p. 47). Therefore, on this model, the self is 
represented in thought and memory as a central and independent node (Kihlstrom, et al., 1988) or space (Markus \& Sentis, 1982) within the individual's network of schemata:

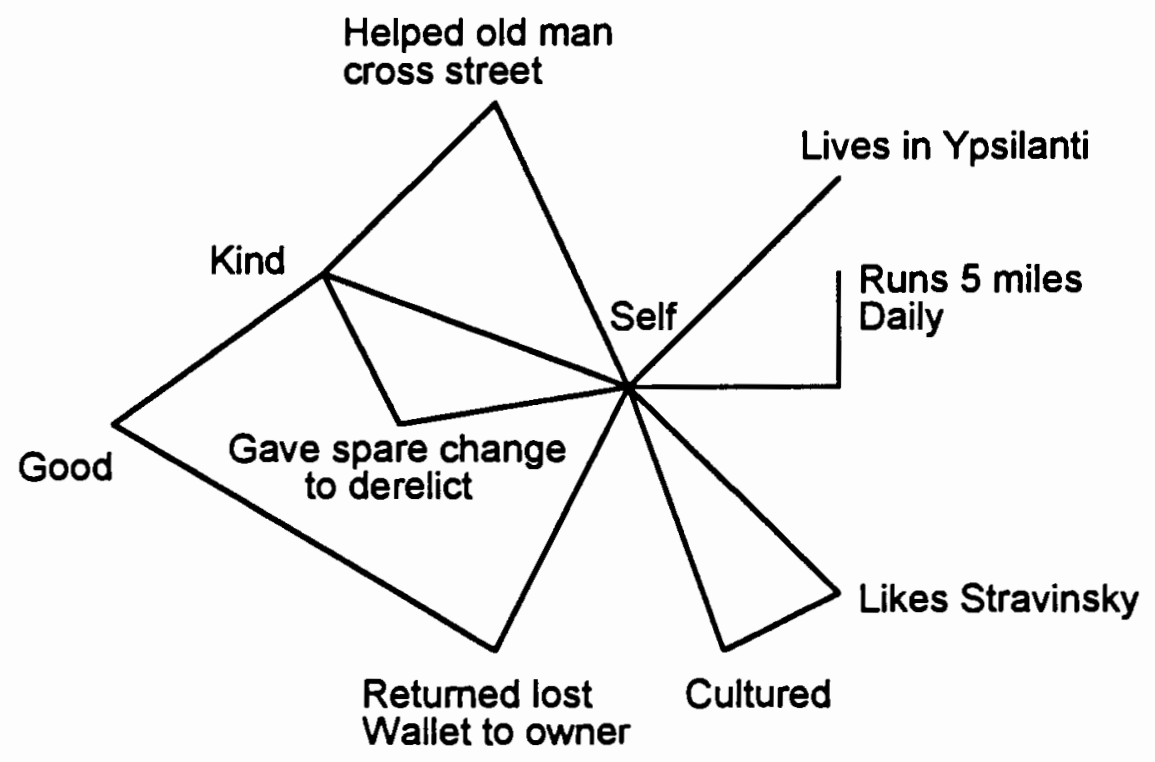

Figure 2. Kihlstrom et. al's conceptualization of self as a node within memory

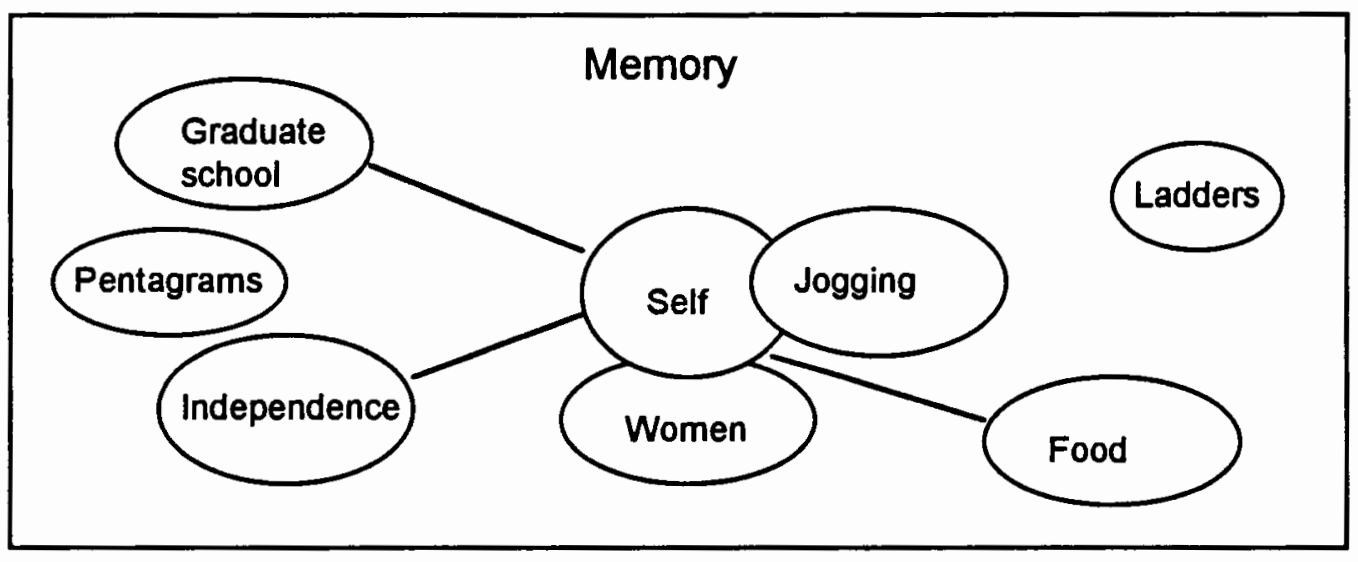

Figure 3. Markus and Sentis's conceptualization of self as a space within memory 
The development of self-schemata does not take place in a social vacuum. As a result, self-schemata also have a strong affective content (Fiske \& Taylor, 1991; Markus \& Sentis, 1982). Just as the opinions of others often play an important role in our views of other people, they also play an important role in our own opinions of ourselves. How an individual perceives potential judgments of others plays a central role in the process of identity development (Mead, 1934). That is, individuals come to know about, or guess about, the judgments that others around them tend to make; and in return, this affects how they perceive and feel about themselves. In short, one begins to have a sensitivity about whether, how, and in what circumstances one is seen as extroverted, competent, American, Asian, Vietnamese, middle-class, etc.,--as well as how these identifications are judged by significant others. The individual incorporates these perceived judgments into existing self-schemata in a variety of ways, depending on (a) the individual's particular self-construal (Markus \& Kitayama, 1992), (b) the quality and salience of the situation, (c) the importance of the other person(s) involved, and (d) the quality and salience of the particular judgments. In this way, people develop generalizations about those characteristics which are most self-relevant and salient within each domain of their experience and then store these within an organized system of self-schemata (Markus \& Sentis, 1982; Turner, 1987). These issues range over the various topics traditionally addressed by social interactionism (e.g., Mead, 1934; Blummer, 1969), personal construct theory (see Bannister \& Fransella, 1971; Kelly, 1955; Weinreich, 1980), and attribution theory (e.g., Heider, 1958). Though 
many of these issues are potentially relevant, a more detailed consideration of them is beyond the needs of this review.

\subsection{Identity Development: Levels of Identity}

Individual models of identity have emphasized that social context plays an important role in identity development. As William James (1890) put it: "Properly speaking a man has as many social selves as there are individuals who recognize him and carry an image of him in their mind" (p. 294). At the same time, individuals don't identify with all others equally. Therefore, not all influences have an equal affect on the formation of the self. From this perspective then, social identity formation is seen as an interplay between increasing differentiation of the world and self and a selective identification with these influences (Allen, Wilder, \& Atkinson, 1985; Berger, 1982; Deveraux, 1982; Turner, 1987).

Generally, the content of this differentiated self-structure is comprised of a variety of elements along a continuum from most to least psychologically universal, or inclusive. That is, differentiations are structured into various levels of generality or abstraction (Allen, Wilder, \& Atkinson, 1985; Turner, 1987). A summary of these levels of development appears in figure 4.

On one end, there are most probably certain universal core identity elements, or categories of self-schemata, affected by relationships with the individual's most significant others during early development (Markus \& Sentis, 1982, p. 48; Vaughn, 1987). These form a "superordinate level" (Turner, 1987) probably including schemata 
of one's sense of humanity (Mcguire \& McGuire, 1982; Tumer, 1987), physical appearance (Allen, Wider, \& Atkinson, 1990), gender (Markus \& Sentis, 1982), name (Ghee, 1990; Markus \& Sentis, 1982), and some form of primary relationship roles-often given in the form of kinship (Kernberg, 1977).

\section{Superordinate level--Universal aspects of human identity.}

\section{Intermediate level--Contextually variable in group / out group identifications.}

\section{Subordinate level--Idiosyncratic aspects of personality.}

Figure 4. Levels of abstraction in identity development

The high level of abstraction in initial identity development theorized by Turner (1987) has been supported by research that shows a salience of various self / nonhuman comparisons in children, such as comparisons with animals, which decrease as development occurs (McGuire \& McGuire, 1982).

On the other end of the identity continuum is a "subordinate level of personal self-categorizations" (Allen, Wilder, Atkinson, 1985; Turner, 1987), in which particularistic self-schemata, not developed by everyone, appear as highly individualistic aspects of personality. As already mentioned, this level of development is very closely related to the types and qualities of an individual's interpersonal contacts. That is, they are developed through interpersonal contact and comparison; and are intimately connected to the type and quality of the interpersonal roles available for the 
individual. In this sense, even particularistic aspects of identity are inherently social in nature.

Between these two extremes is an "intermediate level of in-group/out-group categorizations" (Allen, Wilder, \& Atkinson, 1985; Turner, 1987) involving a number of broad social and cultural schemata necessary for consistent social functioning. These schemata of potential social group identities include categories of nationality, race, ethnicity, regional association, tribal affiliation, class, occupation, etc.,. Therefore, in addition to the level of social identity development which has its source in interpersonal contact, one can also conceptualize social identity on the level of social group membership. Again, as James (1890) put this point: "Properly speaking a man has as many different social selves as there are distinct groups of persons about whose opinion he cares" (p. 294; emphasis in original).

Unlike more superordinate aspects of identity development, the salience of these intermediate identity categories is not universal. Instead, they are choices, or influences, which are socio-cultural in origin (Turner, 1987). On an individual level, these identity categories can be thought of as the particular "ready-made set of endowments and identifications" (Mahapatra, 1983) which are possible for any individual at a given time, place, and family of birth. Very simply, different societies and social contexts present different options for individuals. Just as the situations of birth and individual experience may vary, so may the general social group identifications which tend to be possible or important. Any particular category, or set 
of social group identifications may be important for an individual within a particular social setting, though no particular identifications need be. That is, though the concepts of race, ethnicity, or language group, for example, are often common categories of group identity, they are not universal or necessary categories for everyone. Not everyone develops a racial, ethnic, or language group identity.

Just as social group identity must be distinguished from more superordinate aspects of identity development, it also must be distinguished from particularistic and interpersonal aspects of development. Social group identification is made in reference to a group rather than particular personal attributes or particular significant others (Allen, Wilder, \& Atkinson, 1985; Turner, 1987; Vaughan, 1987). Certainly one's particular network of significant others is important to one's particular group identification (Pradell de Latour, 1983; Heller, 1987); however, this should not obscure the qualitative difference between the two processes. Individual/interpersonal identity and social group identity constitute distinct levels of abstraction within identity development. Therefore, since individual models focus solely on interpersonal comparison and relations, they can offer neither a sufficient description nor sufficient explanation of group identity. Social group categorization is the process by which people are rendered functionally equivalent members of a group of individuals. Intuitively, the difference is apparent in the subjective quality of the respective identity experiences. Feeling that one is very competent or extroverted within a situation, for example, is qualitatively different than feeling that one is very Vietnamese within that 
same situation. That is, self-ascribing attributes of competence or extroversion in a domain need not involve reference to group membership: in feeling extroverted one is not necessarily identifying with the group of extroverted--as opposed to introverted-individuals. However, feeling Vietnamese in a situation necessarily involves the concept of group membership and intergroup contrast. The sense of contrast may be with another specific group such as Cambodians, or with a more general nonVietnamese background. However, "a group identifies itself and is identified by others as constituting a category distinguishable from other categories of the same order ..." (Bart as cited in Mahapatra, 1983; my emphasis). Therefore, group identification always involves judgments of intragroup solidarity and intergroup difference - of which ethnicity is one type.

\subsection{Ethnic Identity}

According to the above view, individuals find a sense of belonging through various levels of social group membership. Several social identity theorists (e.g., De Vos, 1982; Patterson, 1983) have similarly described ethnicity as a reaction to the general existential crisis of isolation and the human need to belong. However, by doing so, these authors have also de-emphasized the particular importance of ethnicity as a special type of identification. On this view, since there are probably not any essential characteristics which define ethnic identification (De Vos, 1982), what matters is the function of ethnic identification within identity development generally. Ethnicity becomes simply one group option among many within the more general process of 
social identity development and maintenance (e.g., Deveraux, 1982; De Vos \& Romanucci-Ross, 1982; Tumer, 1987; Wallman, 1983; Weinrich, 1988).

Central to this view of ethnicity is a recognition of its extensive social variability. This includes variability in the salience of ethnicity as a category of social identity, variability in the basis for the formation of particular ethnicities, and variability in the range and number of ethnicities which can be claimed by individuals in particular circumstances.

Many authors have recognized that the importance of ethnicity as a potential category of social belonging depends on the socio-cultural context (e.g., Chun, 1985; Heller, 1987; Khan et al., 1983; Wallman, 1983; Weinreich, 1982). Ethnicity is not a category of identity for all societies, or even for all groups within a society. Because there are many other group affiliations which provide adequate sources of belonging, not everyone develops an ethnic identity. This is not to discount the phenomenon of ethnicity, but merely to point out that the salience of ethnicity within development should not be treated as a psychologically universal category of group identification. Rather, it is one possibility within a set of potential identity options, or anchors, which vary culturally and socially and are not necessarily shared by every member of a society in the same way or to the same extent.

Given that not everyone develops a sense of ethnic awareness, an important task within the field of ethnic studies has been a description of those factors which are associated with the origin of ethnic identity in general. Extensive research on the 
nature of the "minimal group" (Allen \& Wilder, 1975; Billig \& Tajfel, 1973; Tajfel, 1970; Tajfel \& Billig, 1974; Tajfel, Billig, Bundy, \& Flament., 1971) suggests that perhaps the most important factor is the presence or availability of ethnic group labels. Merely the presence of pre-existing ethnic labels greatly predisposes the individual to recognize ethnicity as an important level of social identity (Turner, 1987; Vaughan, 1987).

A number of other variables also tend to enhance the salience of ethnicity as a potential identity category and push individuals toward particular ethnic identities. Generally, these variables can be divided into two groups: those that increase perceptions of intraethnic similarity and those that increase perceptions of interethnic difference. Many times, the ethnic solidarity of a particular group is enhanced by a shared sense of situation, or crisis (Devereux, 1982; Patterson, 1983). This sense of shared situation may be experienced as a feeling of a common past (Gibson, 1988; Khan et al., 1983; Patterson, 1983), a perception of a shared present situation (see Lopez \& Espiritu's 1990 discussion of pan-ethnicity in the United States), or the belief in a common fate (Khan et al., 1983; for a specific example see Monzel's 1987 discussion of Hmong in the United States).

Identity development is also related to social, political, and ideological factors associated with a consciousness of intergroup differences (Chun, 1985; Keefe, Reck, \& Reck, 1987; Kruwer \& Ekensberger, 1989; Smolicz, 1981b; Weinreich, 1988). These factors include: 
1. The perceived or actual attitudes of the larger society (Devereux, 1982; Lambert, 1990; Patterson, 1983; Smolicz, 1981b).

2. Both the absolute size of the ethnic group and the size of the group in relation to other groups (Patterson, 1983).

3. The level of visibility and distinctiveness of the various groups involved (Lalonde, Taylor, \& Moghaddam, 1992; Moghaddam \& Taylor, 1987; Richmond, 1988)

4. Whether migration is involved as well as the reasons behind the migration (see both Khun, 1983 and Patterson, 1983 for detailed discussions of these issues).

5. The presence of strong two valued attitude systems in either the ethnic group or the larger society which tend to produce strong in/out group categorizations (see Mahapatra's 1983 discussion of Allport).

6. The perceived and actual presence of power differentials between any of the groups involved (Khan, 1983; Patterson, 1983).

7. The social and economic resources available to the various groups (Patterson, 1983).

Any of these factors may act to enhance intergroup differences; and therefore, increase both the salience of ethnicity as a category of identity and the salience of particular ethnicities for particular individuals.

All of these factors emphasize that the development of ethnicity as a potential identity category depends upon an active awareness of culturally different "others" with which to contrast one's own sense of self (Allen, Wilder, \& Atkinson, 1985; De Vos, 1982; Turner, 1985). "One develops a sense of social identity by noticing what one is not" (McGuire as cited in Allen, Wilder, \& Atkinson, 1985, p. 114). Consequently, there can be no sense of ethnicity in an ethnically homogeneous and isolated society. In 
such a case there would only be an awareness of humanity, and non-humanity (DeVos, $1982 \& 1985) .{ }^{27}$ In Bromley's words ethnically anchored individuals have "an awareness of their identity and distinctness (i.e., we-they oppositions) from other similar communities" (as cited in Mahapatra, 1983, p. 61). "Ethnic identity then, is one interplay of self-definition and other definition in [cultural] boundary maintenance" (Mahapatra, 1983, p. 61).

Many social identity theorists have argued that the social variability of ethnicity is reflected in the special ontology of ethnic identity. The peculiar nature of ethnic identity can be seen in the distinction between the internal and external aspects of ethnic consciousness (Patterson, 1983). This dichotomy is what Khan (1983) has referred to as the basic phenomenological problem in the study of ethnicity: We only have access to what people do and say; however, ethnicity cannot be defined merely by external criteria--place of birth, family background, physiognomy, behavior, language, or expressed values (Patterson, 1983; De Vos, 1982). "Ethnicity is also determined by what a person feels about himself" (De Vos, 1982, p. 17; my emphasis). Rather than simply being a commitment to external criteria for group membership, ethnicity is a distinct form of personal consciousness which is independent of any particular external symbol (De Vos, 1982; Khan, 1983). Basic to the definition of ethnicity is this phenomenological dualism between ethnicity as an external set of symbols which express intergroup differences and ethnicity as a form of personal consciousness. 
This is not to say that the internal feeling of ethnicity is completely independent from its external expression. Rather it is to point out the peculiar nature of their connected-ness. Berger and Luckmann (1966) have argued that consciousness in general is constituted by its objects. That is:

Consciousness is always intentional. It always intends or is directed toward objects. We can never apprehend some putative substratum of consciousness as such, only consciousness of something or other. This is so regardless of whether the object of consciousness is as belonging to an external physical world or apprehended as an element of an inward subjective reality.

(Berger \& Luckmann, 1966, p. 20)

Social identity theorists have argued that the same is also true of ethnic consciousness. Ethnicity would be socially meaningless if it couldn't be perceived. Instead, social identity theorists have argued that ethnic identity involves a consciousness of the "objects" and symbols of ethnic differentiation. This is what Khan (1983) has called a "consciousness at the boundary" of interaction between groups.

The connection between ethnic consciousness and ethnic symbolizing has important psychological implications for understanding the personal experience of ethnicity. Individuals who are ethnically anchored often experience ethnic categories as objective aspects of the world into which they are born. This is true of the saliency of ethnicity as a category of identity in general and also how particular ethnicities are experienced: "The ethnic is not only committed to his own particular ethnicity; he strongly believes that all human beings are inherently ethnic, whether they know it or not, whether they like it or not" (Patterson, 1983, p. 29). ${ }^{28}$ In a similar way, the specific objects of ethnic consciousness are often experienced as objectively connected to a 
particular ethnic identity; and as such, any loss or change in the particular symbols of that ethnicity may be experienced as a completely debilitating sense of alienation (see the Catalan example above, p. 14). ${ }^{29}$

On the other hand, the particular external symbols of one's ethnic identity are also, in an important sense, arbitrarily connected to one's ethnicity. As pointed out above, a socio-historical approach to ethnicity reveals that the objects of ethnic consciousness are highly variable across geography and time. Psychologists have emphasized that the contents of one's individual consciousness change constantly within day-to-day experience without necessarily producing differences in one's basic sense of self--continuity in individual identity doesn't necessarily imply sameness. Similarly, social identity theorists have emphasized that changes in the objects of ethnic consciousness (even for a particular group) don't necessarily result in differences in ethnic identity. Whether changes in an external symbol results in a change in identity is an empirical matter that can only be answered in reference to the group involved.

This would suggest that one should take a functional psycho-cultural approach (De Vos, 1982). That is, one must distinguish the need to differentiate from the need for the particular symbols used to do so (De Vos, 1982). Particular symbols (such as the use of a particular language) are not inherently connected to particular ethnic identities. They are associated with a particular identity only in so far as they function to (a) focus group solidarity for particular purposes, and (b) distinguish that group as unique from other groups within particular contexts. As social contexts change 
through history or because of migration, the particular symbols which function to distinguish groups from one another often change as well. In this sense, though there can be a theory of identity which may be generalizable to ethnicity, there can not be a universal theory of ethnicity itself (Wallman, 1983; Weinreich, 1988). As De Vos (1982) simply comments: "If one seeks to define those characteristics that define an ethnic group, one ultimately discovers that there are no essential characteristics common to all groups so designated" ( p. 9).

Using a psycho-functional approach, one would expect that once ethnicity as a level of identity is activated, the need to differentiate will tend to be fairly stable. However, the particular symbols used to differentiate a particular ethnicity may vary widely according to both the particular group and the particular context in which the group finds itself.

This dialectic between the external and internal aspects of ethnicity is often at the root of disagreements between generations or geographically separated subgroups over the importance of maintaining particular ethnic symbol. For instance, many older immigrant Vietnamese may have felt a personal sense of alienation in the process of behavioral acculturation when they migrated to the United States. Many of them may worry that the changing behavior of younger Vietnamese within the United States is a sign that their children are losing their Vietnamese identity. However, it may also be true that those younger Vietnamese continue to feel just as Vietnamese, but have simply shifted the particular symbols in which they express that Vietnamese-ness. 
Similarly many Vietnamese in the homeland might not describe young VietnameseAmericans as "Vietnamese," while at the same time those young Vietnamese continue to feel, and identify themselves as, very definitely Vietnamese. On this approach, questions of what type of attributes are "really" Vietnamese are meaningless, for the particular attributes and symbols which function to maintain ethnic uniqueness naturally change according to the context. What really counts is how individuals feel about themselves.

Heller (1987) has argued that the basis for ethnic identity is found in an individual's participation in ethnic social and communicative networks. Given this description, it is understandable how individuals in multi-ethnic societies often develop multiple ethnic identifications. Since many people play a variety of roles in a variety of different social networks--some of them ethnically different--they often develop a variety of flexible selves to match the variety of particular circumstances in which they are involved (Allen, Wilder \& Atkinson, 1985; Berger \& Badac, 1982; De Vos, 1985; Turner, 1987). An individual is constrained in particular situations by (a) the range of potential ethnic identifications available, and (b) the variety of social and political factors which determine that individual's real and perceived access to the various ethnic networks. However, rarely are individuals constrained to only one option. It is important to recognize that when individuals are given access to a variety of group identities, it is common for them to develop a number of ethnic identities of varying levels and strengths (Carstens, 1986; Clammer, 1986; Khan, 1983; Kruwer \& 
Ekensberger, 1989; Wallman, 1983). For example, if one is Chinese-VietnameseAfrican American, it may be natural to identify one's self as Chinese in one situation and Vietnamese in another. In still other situations pressing to be recognized as AfricanAmerican, African, Asian, or Southeast Asian might be best; in still other contexts it might be best to identify one's self as a "person of color," Vietnamese-American, or American. One individual may claim any, or all, of these identifications without necessarily feeling any sense of internal conflict.

Multiple ethnic identification also has important methodological implications. Researchers have rarely given their research subjects the chance to identify themselves with more than one ethnic group. However, doing so may result in a more valid reflection of subjects' actual experiences. For example, when respondents in a study by Baker (1982; as cited in Wallman, 1983) were given the chance to choose more than one ethnic identity, the vast majority of respondents chose more than one. These results suggest that the possibility of multiple identifications as well as the particular contextual constraints on the range of identities available should always be considered in the design and operationalization of any measurement of ethnic identity (Wallman, 1983). If multiple identifications are not considered, then the possibility that "the findings might be embedded more in the forced-choice methodologies than in the attitudes of . . [subjects]" (Morgan, 1991, p. 248), will severely weaken or invalidate any results. ${ }^{30}$ 
The above discussion suggests that the salience of (a) any level of categorization (i.e., social class, ethnic, racial, national, etc.); (b) any particular identification from any one level of categorization (Vietnamese, Chinese, VietnameseAmerican, Asian, etc.,); and (c) the basis for any particular identification (i.e., shared sense of past, present, future, common enemy, common characteristics, etc.,) are highly variable and context dependent. Moreover, ethnicity is not always experienced as a discrete and fixed pattern of identity choices. For many groups and in many contexts, ethnicity can also be multiple and highly negotiable (for one example of how complex ethnic negotiation can be, see Carsten's 1983 \& 1986 discussions of the Hakka Chinese in Malaysia).

The contextually negotiable nature of ethnicity highlights the active aspects of personal and collective choice involved. This would suggest that ethnicities are not accepted or developed passively. They are actively chosen, rejected, and negotiated. The active role that the individual plays in the development of ethnicity has been recognized in theories of ethnic awareness and commitment (Atkinson, Morten, \& Sue, 1983; Marcia, 1980; Phinney, 1989) as well as several orthogonal theories of acculturation (e.g., Berry's, 1986 multidimensional theory of acculturation). 


\subsection{Ethnic Identity and Language.}

Taken collectively, the above ideas form the basis of a framework for addressing the relationship between ethnic identity and language choice. Within this framework, a particular ethnic identity (one's identification with a particular ethnic group) or a particular language identity (one's sense of cultural "ownership" of a particular language) are two distinct constructs which may or may not overlap. Further, the manner in which they overlap may vary depending on the social context. As a result, the relationship between these two constructs is conceptualized as a set of possible structural configurations. That is, there is nothing within the content of any particular ethnic identification and any particular language identification which makes them either inherently connected or inherently incompatible. Given the appropriate context any language could be used to express any particular ethnic identity. On this view, an exclusive connection between a language and an ethnicity is simply one possible relationship. However, exclusivity is only one of several possibilities. The particular group of ethnic and language identities that individuals feel are available to them at a particular time and place define a set of logically possible identity arrangements, any one of which could guide identity development given the right set of conditions.

To begin delineating the general types of self-construals which are relevant, I will consider a bicultural / bilingual model. However, the conceptual direction of my description could be extended to cover more complex multicultural / multilingual 
situations. It could also be extended to include situations in which either ethnicity or language lack self-salience. Though these situations are possible, they would add undue complexity to the present description. As a first step in this process, I present the following logical possibilities in Table I:

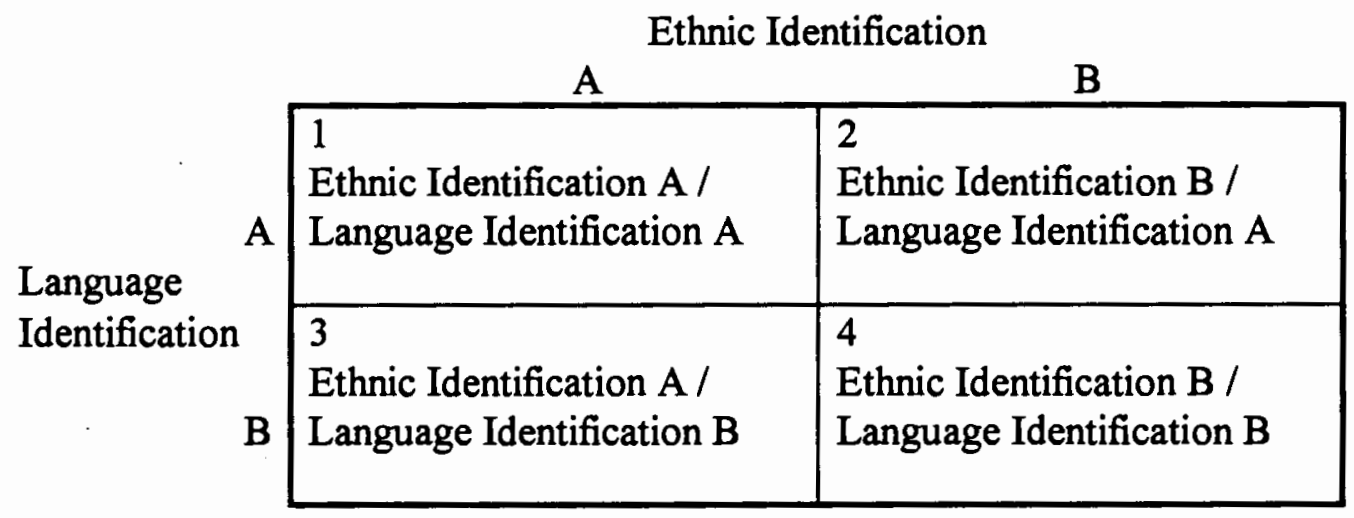

Table I. Matrix of relationships between ethnic identity and language identification

If one focuses on the structural aspects of these choices, then the above table can actually be collapsed into two categories. Choices 1 and 4 can be collapsed into a single category which I will call linguicultural monism; and choices 2 and 3 can be collapsed into a category which I will call linguicultural transference (Figure 5). Choices 1 and 4 represent a state in which individuals feel that their particular ethnocultural identities are most naturally and comfortably expressed (lived) in a traditionally associated language. Choices 2 and 3 represent a situation in which individuals feel most comfortable expressing their ethno-cultural identity in a language that is not traditionally associated with that identity. 
1. Ethnic Identification A / Language Identification A (e.g., Feel Vietnamese / in Vietnamese)

4. Ethnic Identification $B$ / Language Identification B (e.g., Feel American / in English)

Linguicultural Monism

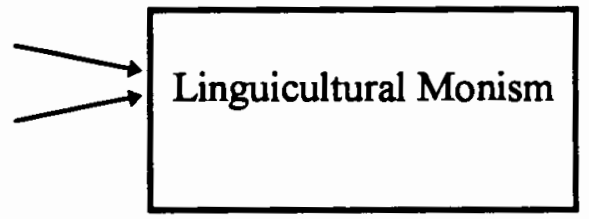

2. Ethnic Identification A / Language Identification B (e.g., Feel Vietnamese / in English)

3. Ethnic Identification B / Language Identification A (e.g., Feel American / in Vietnamese)

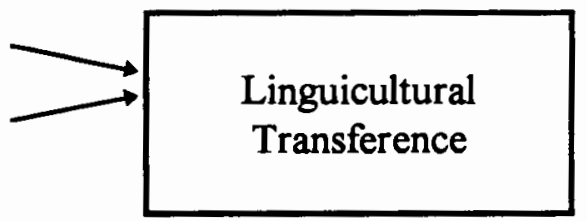

Figure 5. Monism and transference

Linguicultural monism represents an identity construal in which there is a strong psychological association between a particular ethnic identity and the use of an associated language-- often times considered THE mother tongue. This type of identity-language construal is common in groups for whom language is a core cultural value (Smolicz, 1980 \& 1981a). For individuals in this category, specific ethno-cultural identities seem somehow "naturally" connected to a specific range of linguistic behaviors, attitudes, and values. As a result their attitude towards language learning tends to be either strongly integrative or instrumental. These individuals feel that becoming a member of the particular ethnic community necessarily involves use of the associated language and they also feel that learning to speak that language necessarily involves acculturating. Therefore, individuals who are socialized in a different language tend to be treated as "foreign" and to the extent that immigrants either refuse, or are unable, to learn the required language behavior, they also tend to be seen as outsiders. Conversely, individuals with a monistic self-construal often associate using an additional language with varying degrees of personal alienation. This feeling may 
even be generalized to the point that these individuals feel that the disappearance of their mother tongue would mean nothing less than the virtual disappearance of their ethnic group. On the group level, these assumptions are associated with what Chan and Tong (1992) have described as a primordialist view of ethnicity. Primordialists feel a strong connection between several key concepts. They tend to feel that race, ethnicity, culture, and language are unified within, and synonymous with, a strong homogeneous concept of nationalism. Mahapatra (1983) has formulated this often "traditional" concept as "the proposition that a race is equal to a culture and a culture is equal to a language and that a society is equal to a unit which rejects [. . ] others" (Mahapatra, 1983, p. 61). In these groups social identity is maintained by a normative ideology of cultural and linguistic coalescence. That is, in situations of linguicultural monism, individuals' social identities are:

... understood not only in terms of what they are and what they will be, but what they should be. .... What is prescribed here is a vision of one country, one culture, one ideology, one way of feeling, thinking and doing--a loop back into tribal existence of oneness and homogeneity.

(Chan \& Tong, 1992, p. 141)

Language homogeneity and standardization become both expressions and instruments of this ideology of monism. In such "compact homogeneous groups a participant shares maximum verbal experience with other members of the group and is bound by feelings of group solidarity (Khubchandani, 1983, p. 29). Language homogeneity both expresses and creates this solidarity. Khubchandani (1983) argues that in small homogeneous groups the pressure for language standardization begins in the implicit 
forces of group identification. However, as these groups develop greater diversity and have increased contact with other groups, the processes of standardization become explicit instruments of identity circumscription.

... language boundaries become sacred and spontaneity and creativity leading to hybridization are filtered through the standardization process. In such situations, the contextual and functional fluidity in speech which manages to cross the boundaries of language and diction, is depreciated by the custodians of language.

(Khubchandani, 1983, p. 29)

Standardization of language, in this sense, can be regarded as a legitimizing activity expanding its institutional order through a programmed course in socialization" (Berger and Luckmann, as cited in Khubchandani, 1983, p. 28).

In contrast, linguicultural transference consists of transferring one's identity into a language or languages which are different from the historical mother tongue for that identity. The state of English in many Outer Circle countries can be described in terms of transference. Since I have described this in detail within section 2.0 , I will only make a few additional comments here. Transference reflects an identity construal in which there is a weak association between ethnic identity and a mother tongue. Unlike communities of linguicultural monism, these communities don't expect as high a level of language acculturation from other culturally different individuals. Similarly, when these individuals use a language other than their mother tongue they do not necessarily associate the experience with extreme levels of alienation. Sometimes this situation arises because language is not a core cultural value for the community. Other times, transference develops from post-colonial situations in which the colonial language 
remains after independence. ${ }^{31}$ In some of these cases, the old colonial language authority begins to wane and the colonial language begins to be nativized to express local content, communication patterns, and needs. On an individual level, this situation is associated with what Pride (1979) has called an expressive motive: the motivation to learn another language "in order to express elements of one's own culture" (p.54).

The possible identity arrangements in table 1 can also be expanded to produce several other possibilities for multilingualism. If one ignores those combinations which are strict contradictions, ${ }^{32}$ two other possibilities are present. Categories 1 and 4 can be combined to form a state of linguicultural bifurcation and 2 and 3 can be combined to form a state of bi-directional transference and integration.

$$
1
$$

Ethnic identity A/

Language identity $\mathbf{A}$

(Feel Vietnamese/

Speak Vietnamese)
4

Ethnic identity B /

Language identity $B$ (Feel American/

Speak English)
Linguicultural Bifurcation

L

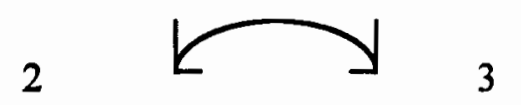

Ethnic identity A/ Ethnic identity B / Language identity $B \quad \& \quad$ Language identity A (Feel Vietnamese/ Speak English) (Feel American/ Speak Vietnamese)

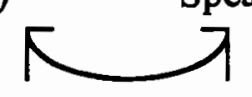

Linguicultural integration

Figure 6. Bifurcation and integration

Linguicultural bifurcation represents a state of individual level diglossia and integration represents a fluid and mixed state of individual multilingual multiculturalism--what Bennett (1993) has referred to on the individual level as 
"constructive marginality". ${ }^{33}$ The isolation of the two identities within bifurcation is represented in Figure 6 by the vertical line between the two sides of the structure and the bi-directional influence present in a state of integration is represented by the circular arrows connecting the two sides of the bottom structure.

The concept of linguicultural bifurcation is present in many traditional models of bilingualism. On this view, the relationship between each language-ethnicity pair is conceptualized in a manner consistent with linguicultural monism; however, in bifurcation the individual contains two separate linguistic-cultural identities. This conceptualization of bilingualism as bifurcation is captured in several authors' emphasis on the cultural duality of bilinguals (Agar, 1991; Guiora, 1972 \& 1976; Heller, 1987; Northover, 1988; Weinreich, 1988; Preston, 1989); Both Agar's emphasis on "The biculture in bilingual" (1991, p. 111) and Weinreich's definition of bilinguals as "dual cultural-linguistic identities" (Weinreich, 1988, p. 207) are consistent with this conceptualization. As Weinreich states:

In the case of bilinguals . . each language binds the user to a particular set of cultural beliefs and values associated with that language and that each of a bilingual's languages is the mediator between two differing cultural identities within one and the same person. (Northover, 1988, p. 207; my emphasis) Heller (1987) argues similarly that bilinguals possess two codes which are functionally and symbolically bifurcated between the two distinct ethno-cultural communities in which bilinguals operate. As a result, this view naturally emphasizes a coordinating motive in which "the learner regards his task as that of acquiring the ability to code- 
switch in an appropriate and orderly manner" (Pride, 1979, p. 56). This motive naturally highlights the linguistic phenomenon of “'correlational' or 'situational' code switching (standard French in his office . .., standard Dutch in his club . ...)" (Pride, 1979, p. 56).

On the other hand, linguicultural integration represents an identity state of bidirectional transference. In this process, the two ethno-cultural identities are integrated in varying degrees-what Pride (1979) has referred to as a "blending motive." Often associated with integration is a high amount of linguistic code mixing in which the various codes are integrated on all levels of use. Rather than developing linguistic homogeneity and standardization, these groups tend to develop what Khubchandani (1983) has referred to as "organic" or "folk" multilingualism. Khubchandani has contrasted organic multilingualism with the "elegant" multilingualism of societal diglossia. Elegant multilingualism--what I have described as bifurcation--is the product of an ideology of language separation and standardization, and is produced through standardized education: "Through different standardization techniques, the self-evident situational affirmations give way to formalized explicit procedures, as prescribed by the officially accredited custodians of linguistic practice in grammar manuals, dictionaries, style sheets and so on" (Khubchandani, 1983, p. 28). In contrast, folk multilingualism represents a state of open fluidity, non-congruence, and crisscrossing of identity and language affiliations (Khubchandani, 1983, p. 9). The result of this fluidity is a fuzzy diversity in [the] everyday repertoire" (Khubchandani, 1983, p. 25) of cultural, 
linguistic, and functional heterogeneity. Individuals who grow up in conditions of folk multilingualism often show ambiguity in mother tongue identification and language identification generally. Hutton has commented on mother tongue ambiguity in the 1931 census report of India:

So deep does bilingualism go in parts of Ganjam that from very infancy many grow up speaking both Oriya and Telugu, and are so much at home in both that they can not tell which to return to as their mother tongue.

(as cited in Khubchandani, 1983, p. 9)

On looking at the census process within India, Khubchandani has commented that "this phenomenon of plurality of mother tongues is much more widespread than has been recorded in linguistic studies ...." (Khubchandani, 1983, p. 9). Mother tongue ambiguity is just one aspect of the high level of ambiguity in language identification within folk multilingualism generally.

In such conditions, natives speak a kind of "language" which defies the standard notion of "grammar." People do not associate speech labels precisely with grammatical and pronunciation stereotypes, and standardization and other propriety types of control of verbal behavior generally tend to be permissive. Consequently it is often difficult to determine whether a particular discourse belongs to language $\mathrm{A}$ or language $\mathrm{B}$.

$$
\text { (Khubchandani, 1983, p. 9) }
$$

The choices of bifurcation and integration can be added to monism and transference to form an expanded description of possible identity / language construals. This expanded description of choices can be summarized in Table II. The first term in each cell within the matrix describes an individual level identity-language state and the following terms describe the associated identity-language processes which individuals tend to adopt when placed in a social environment that is dominated by the assumptions 


\begin{tabular}{|c|c|c|c|}
\hline & & Unidirectional & crete $\longrightarrow$ Bi-directional \\
\hline $\begin{array}{l}\text { Association } \\
\text { between } \\
\text { specific } \\
\text { ethnic }\end{array}$ & Strong & $\begin{array}{l}1 \\
\text { Linguicultural monism } \\
\text { Assimilation/Accommodation } \\
\text { Integrative / instrumental } \\
\text { Language standardization } \\
\text { Interlanguage } \\
\text { Interference } \\
\text { Transitional bilingualism }\end{array}$ & $\begin{array}{l}2 \\
\text { Linguicultural bifurcation } \\
\text { Elegant multilingualism } \\
\text { Coordinating motive } \\
\text { Emphasis on autonomy of code } \\
\text { Code switching } \\
\text { Language Standardization } \\
\text { Interlanguage } \\
\text { Interference } \\
\text { Maintenance bilingualism } \\
\end{array}$ \\
\hline $\begin{array}{l}\text { and } \\
\text { specific } \\
\text { language } \\
\text { identities. }\end{array}$ & Weak & $\begin{array}{l}3 \\
\text { Linguicultural transference } \\
\text { Nativization } \\
\text { Expressive motive } \\
\text { Functional cline of sociolects } \\
\text { Code mixing--w/ received code } \\
\text { Linguistic imperialism }\end{array}$ & $\begin{array}{l}4 \\
\text { Linguicultural integration } \\
\text { Folk multilingualism } \\
\text { Blending motive } \\
\text { Code mixing } \\
\text { Nativization } \\
\text { Functional fluidity } \\
\text { Mother tongue ambiguity } \\
\text { Hierarchical complementation }\end{array}$ \\
\hline
\end{tabular}


of that category. Since there is a strong association between specific ethnic identities and specific language identities in the upper half of the table the relationship between 1 \& 2 can be conceptualized as a discrete, additive process of combining distinct unitary cultural / linguistic units. Conversely, because there is a weak relationship between specific ethnic and language identities in the lower half of the table, the relationship between 3 and 4 should be conceptualized as a non-discrete continuum.

A schema model of identity can be used to understand how these different categories of relationships are represented within individual psychology. On an individual level, each of these different types of relationships can be conceptualized as four different structural configurations of self-schemata. Using a spatial model similar to those presented in section 3.1 .4 , (pp. 35-39), various schemata and groups of schemata (e.g., ethnic identity, being a speaker of a particular language, etc.) are represented as points or spaces in a structured identity map. In such a map, the relative self-salience of various schemata are represented by their relative distances from a central point within the self-space. Adopting this representation, the four categories presented above can be conceptualized as different structural relationships between the specific schemata related to ethnic and language identities. The basic description of each identity construal is the same as presented above. However, this alternative representation links the description of these identity states to the earlier conceptualization of the self as a system of self-schemata and to the Multidimensional scaling (MDS) methodology described in the next section. Therefore, conceptualizing 
identity as a structured space of self-schemata links theory and method within a completely consistent framework. ${ }^{34}$

Very briefly, I will describe each identity construal in relation to an associated "identity map." As above, I will use a general bilingual model and present each self-construal as an idealized case. An identity state of linguicultural monism (Figure 7) is constituted by a strong overlap between the schemata associated with a specific ethnicity and a specific language.
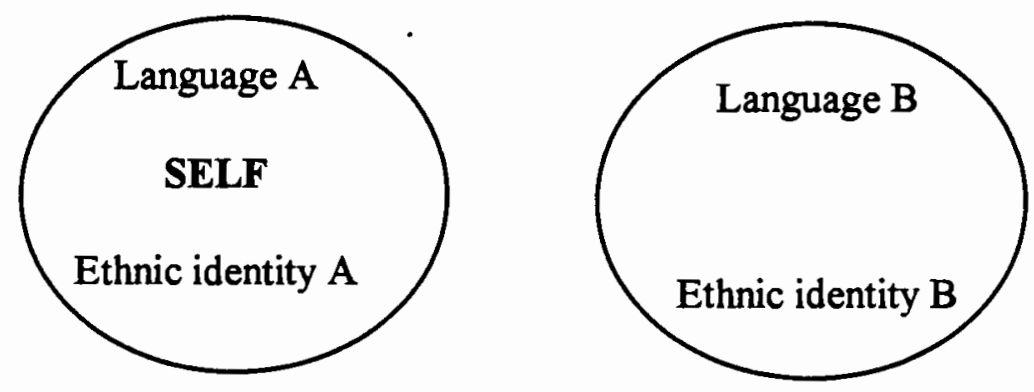

Figure 7. Schema structure of monism

In this state, the individual has an obviously primary ethno-cultural identity which is strongly associated with a particular language. Other languages are experienced as "foreign" to this identity and therefore second language learning is often associated with psychological stress. If an integrative motive dominates, this stress is often times resolved through bifurcation. Another solution, is to adopt an instrumental attitude. In instrumental language use, the second language users neither connect the language to their ethno-cultural identity nor use the second language for the purpose of taking on a new cultural identity. As a result, the additional language-culture system 
remains only minimally self relevant. It should be pointed out that instrumental use can also extend to the use of an individual's ethnic mother tongue. This may arise, for example in situations where there are clear majority/minority relationships within a country. In these cases, some individuals may associate their identity more with the dominant language than their mother tongue, though they continue to use their mother tongue for practical purposes within the mother tongue communities.

In a bifurcated identity state, there are two distinct systems of schemata-both of which are equally self-relevant. Figure 8, presents the two language culture systems as equally developed. This description of two monolingual competencies in one

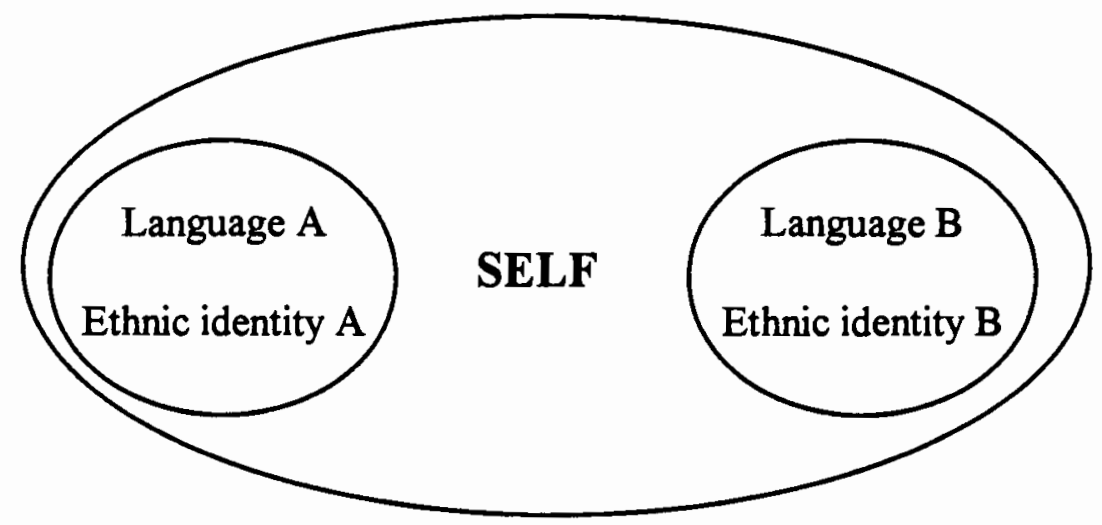

Figure 8. Schema structure of bifurcation

person is an implicit ideal in many discussions of bilingualism. However, in actuality both the level of development for each system and the relative level of self-salience of the two systems varies according to the social importance which is attributed to each. As mentioned earlier, the stability of a bifurcated state also depends on the individual's 
ability to keep the two identity systems separated in actual use. Therefore, this state is often associated with domain-specific language use. That is, the various languages begin to fulfill different symbolic and functional roles which are associated with different identities and contexts.

Linguicultural transference (Figure 9) is the result of cross-cultural linguistic influence. In this situation a language becomes dominant in a new cultural context, and becomes uniquely nativized to express its new cultural content. Within the individual's identity, this new cultural ownership results in the indigenous ethnic identity becoming associated with the use of the new language as much (sometimes even more) than the indigenous language.

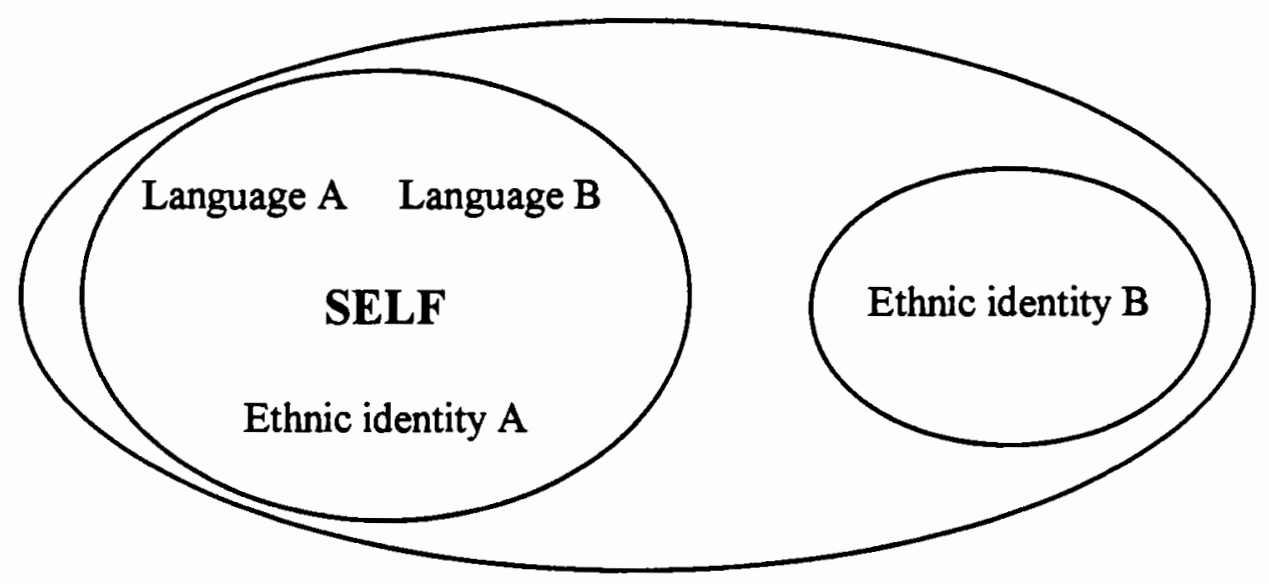

Figure 9. Schema structure of transference

In contrast to a state of monism, the "non-mother tongue" or "second" language is strongly connected with the user's indigenous ethnic identity and is either 
closer (i.e., more self-salient) or equally close to the self to the self than the traditional "mother tongue."

Linguicultural integration results from community-wide fluidity in identity and language networks which are only minimally affected by any pressures for standardization. As a result, individuals tend to exhibit mother tongue ambiguity, low awareness of language differences, highly negotiable ethnicity relations, and extreme levels of code mixing. However, precisely because this state is characterized by such high levels of fluidity in ethnic and language identification, it is not adequately represented by a static map. ${ }^{35}$ Further, because it requires community-wide assumptions which are simply not characteristic of the US context, it is not relevant for the present study. Therefore, I will leave its representation unspecified.

This presented conceptualization is relevant to the present study of identity and language within the Vietnamese-American community in the following way. The literature presents several possible trends in how the relationship between language and ethnic identity might develop within the Vietnamese-American community. These descriptions roughly correspond to the three categories of monism, bifurcation, and transference. When operationalized using a multidimensional scaling (MDS) methodology (see section 4.0, p. 70), the present conceptualization provides a rigorous way to describe and characterize the flexible range of identity relations which may be involved. Therefore, the main concern of this research is as follows. If one could produce a map of the identity relations for a group of Vietnamese-Americans, how 
would this map look? First, what descriptions would constitute the content of this map? Second, what basic categories of identity would be involved? Third, what would be the major dimensions underlying the map's structure? Lastly, how would the various ethnic and language descriptions be structured in relation to both each other and to the self? Lastly, how do various contexts which are potentially relevant to ethnic identity and language affect the pattern of self-salience exhibited in these maps? Any of these questions are open for exploration when the conceptualization is fully operationalized. 


\subsection{Methodology}

The following description will present (a) a summary of the conceptualization of the research question; (b) a summary of the procedures which were used; (c) a general description of cluster analysis and multidimensional scaling (MDS); (d) a description of the specific MDS procedures which were chosen for the proposed question under study; and (e) a discussion of the appropriateness of a MDS approach for the topic under study.

\subsection{Summary of Conceptualization and Procedures}

\subsubsection{Summary of Conceptualization}

The present research used multidimensional scaling (MDS) methods to describe the relationship between the subjects' ethnic and language identities. I designed the research in such a way as to leave open the possibility for subjects to report mixed language and ethnic-cultural identities without assuming in the design that these identities were incongruous, unhealthy, inherently unstable, or necessarily transitional. In Table III, I offer the following grid of possible structural arrangements for two potential categories of identity labels which (among others) could have appeared in the results. 
Ethnic Identification

\begin{tabular}{|c|c|c|}
\hline & A & B \\
\hline A & $\begin{array}{l}\text { 1. (Monism) } \\
\text { Ethnic Identification A / } \\
\text { Language Identification A }\end{array}$ & $\begin{array}{l}\text { 2. (Transference) } \\
\text { Ethnic Identification B / } \\
\text { Language Identification A }\end{array}$ \\
\hline Identification & $\begin{array}{l}\text { 3. (Bifurcation) } \\
\text { Ethnic Identification A / } \\
\text { Language Identification B }\end{array}$ & $\begin{array}{l}\text { 4. (Integration) } \\
\text { Ethnic Identification B / } \\
\text { Language Identification B }\end{array}$ \\
\hline
\end{tabular}

Table III. Potential identity construals for Vietnamese Americans

Many studies of ethnicity and language choice assume that only categories of $1 \& 2$ are possible. Other studies allow the possibility for $2 \& 3$, but treat them as inherently dysfunctional and therefore, transitional (Berry, 1986; Weinreich, 1988; Northover, 1988). Earlier, I have labeled these beliefs as assumptions of linguicultural monism-the belief that specific ethnic cultural identities are somehow most naturally connected with their "matching" languages and that mixed ethnic-cultural identities and language identities are inherently unstable. As a result many studies have not considered the possible existence of, or psychological advantages of, multiple and mixed ethnic identifications. Neither of these possibilities was ruled out in the design of this study. I couldn't find any reason to out multiple ethnic identifications a priori rule.

Furthermore, nothing in the psychological literature suggests that the mixed categories of 2 or 3 , above, must be dysfunctional. In fact, there is evidence from other countries which suggests that, in the right social context, these identity arrangements can be both functional and creative. 
Given this background, the question of whether some ethnic groups (in this study, young adults, all of whom were born in Vietnam) have multiple or mixed linguistic and cultural identities should be treated as an empirical question. A MDS approach provides a method for addressing these possibilities.

\subsubsection{General Description of the Subjects.}

The target population for the study was adult Vietnamese-Americans all of whom were born in Vietnam, but were either permanent residents or citizens of the US. All of the subjects had at least some schooling in US high schools and were attending either a community college or a four-year university. Since the question under study was whether some Vietnamese-Americans judge their Vietnamese identities as closely related to their English-speaking identities, the present study did not use random sampling procedures. Instead, a convenience sample was used. Volunteers were solicited from sources which would most probably provide subjects with a potentially high level of Vietnamese identification and a high level of English proficiency and frequency of use. Potential subject sources included Vietnamese student organizations within educational settings. However, the data collection was not limited to those settings. More detailed descriptions of the subjects' demographic characteristics are provided in the appropriate sections of the results. 


\subsubsection{Summary of Procedures.}

The present research addressed the relationship between the subjects' ethnic and language identities through the use of self-labels and multidimensional scaling procedures. In particular, I was interested in whether some Vietnamese-Americans judged their Vietnamese identities as equally closely related to their English-speaking identities as their Vietnamese-speaking identities. The target population for the study was adult subjects born in Vietnam, but now living in the United States. Most of the subjects were university-level students. All of the subjects were volunteers who were solicited from sources which had a high probability of providing individuals with both high levels of Vietnamese identification and high levels of English proficiency and frequency of use.

Data collection involved two stages and was collected in both group and individual contexts. First, an over-inclusive list of self-descriptions was generated by using a multiple administration of a modified Twenty Statements Test (Khun and McPartland, 1954; hereafter referred to as TST). The modified TST asked 43 subjects to brainstorm (in writing) up to 20 possible answers to each of the following:

1. Who are you?

2. Who are you ethnically and culturally?

3. What languages are important to understanding who you are?

The answers were single words, phrases, or sentences. After the modified TST, demographic data were collected from the subjects through a survey instrument.

Subjects from the first stage of data collection were also involved in the second stage--though subject selection was not limited to those who participated in the first 
stage. During the second stage, the descriptions generated by the TSTs were sorted into categories of responses using an iterative semantic grouping procedure. These labels were translated and each label was printed on a card--English on one side and Vietnamese on the other. An additional card labeled MYSELF was also translated and included. The subjects sorted these cards into piles on the basis of perceived similarity, using as many piles as they wished. In addition, subjects were also asked to separately select five cards which they believed were most important for six specific domains.

The individual sorts were combined into aggregate similarity matrices for both the group as a whole and various subgroups within the subject population. A non-metric multidimensional scaling analysis and a hierarchical clustering analysis were performed on the matrices. The resulting maps were interpreted by considering the clustering of the labels, the possible meanings of each map's dimensions, and the relative positioning of the labels and clusters within each map. Finally, the frequencies of labels chosen for each domain were used to examine the relative salience of the underlying dimensions for those domains.

\subsection{What is Multidimensional Scaling?}

This section presents a basic introduction to multidimensional scaling. It is meant for those readers who are not familiar with MDS. Therefore, those readers who are already familiar with the procedure may wish to continue reading at section 4.3.

MDS is a method for producing a map of respondents' judgments of similarity or preference among a set of perceptual or mental objects. The potential objects 
involved may be specific physical objects such as consumer products; types or categories of objects (e.g., cars vs. mini-vans; fruits vs. vegetables); different brands of objects (e.g., Ford cars vs. Toyota); or the brand names themselves. Possible objects may also include people; characteristics of people; categories and groups of people (e.g., nationalities, ethnicities) as well as corporations (e.g., IBM versus ITT). Finally, MDS can be used to assess ideas and concepts generally (e.g., the concepts associated with knowledge vs. wisdom). In short, anything that can be either perceptually processed or stored in memory can be assessed using MDS. Very often MDS is used to determine "the number of dimensions respondents use when evaluating objects" (Hare, Anderson, \& Tatham, 1992, p. 317); to "provide information on the perceived relationships among objects ${ }^{36}$ when the underlying dimensions of evaluation are not known" (Dillon \& Goldstein, 1984, p. 108); and to identify "which dimensions respondents use when evaluating [those]objects" (Hare, et. al., 1992, p. 317). Dillon and Goldstein (1984) list the following applications, among others, for which MDS has been used:

1. Political scientists have used MDS to understand voters' perceptions of political candidates.

2. Anthropologists have used MDS in the study of cultural differences.

3. Psychologists have used MDS for the study of individual and group differences in perception and personality.

4. Sociologists have used MDS to determine perceptions of organizational structure.

Especially relevant for this study was research that had used MDS to study personality concepts (Rosenberg \& Sedlak, 1972; Rosenburg, Nelson, \& Vivekanthan, 
1968; Shweder \& Bourne, 1984), the ethnic identities of French and English Canadians (Taylor, Bassili \& Aboud, 1972 \& 1973), the ethnic and language identities of Welsh versus non-Welsh in England (Giles, Taylor, \& Bourhis, 1974; Giles, Taylor, Lambert, \& Albert, 1975; see Taylor, 1975 for a summary of these studies), and the development of ethnic identity among a culturally diverse set of grade schoolers in Canada (Aboud \& Christian, 1979).

Generally, respondents are asked for judgments of either relative similarity or relative preference between a set of objects or concepts. This process results in a set of perceived distances between all the particular objects involved--usually given in the form of a matrix of relative distances. Then, in much the same way that one could reconstruct a geographical map of cities from a table of all the relative distances between those cities, MDS constructs a geometric map of perceived similarity or preference from the table of respondents' similarity or preference judgments. The resulting map attempts to model the psychological distances within geometric space such that:

If objects $A$ and $B$ are judged by respondents to be most similar compared with all other possible pairs of objects, MDS techniques will position objects $A$ and $B$ in such a way that the distance between them in multidimensional space is smaller than the distance between all other pairs of objects.

$$
\text { (Hare, et. al., 1992, p. 320) }
$$

When the process is completed "the resulting perceptual maps show the relative positioning of all objects in a geometric space" such that the relative distances in that space are analogous to the perceptual distances shown in the respondents' judgments. 
Very generally, MDS attempts to model the distances in the given data within a number of dimensions commensurate with those latent in the data--each dimension being representative of a criterion of judgment used in the comparison process.

For illustrative purposes, the distances between cities provide a clear example of the technical procedures involved in MDS. Table IV presents a matrix of distance data between several cities (the example of mileages between cities is used by Johnson \& Wichern, 1982; and Norušis, 1992; Rosenberg \& Sedlak, 1972).

This matrix is analogous to the similarity or preference data which are normally collected from subjects. Given such data, the purpose of MDS would be to construct a map of the relative locations of these cities from the data in the matrix.

\begin{tabular}{|c|c|c|c|c|c|c|c|c|c|}
\hline & NY. & Chica & Miam & LA. & SF & N. Orl & Wash & Denv & Dalla \\
\hline NY. & 0 & & & & & & & & \\
\hline Chicago & 818 & 0 & & & & & & & \\
\hline Miami & 1325 & 1386 & 0 & & & & & & \\
\hline LA. & 2911 & 2189 & 2885 & 0 & & & & & \\
\hline San Francisco & 3082 & 2233 & 3239 & 107 & 0 & & & & \\
\hline New Orleans & 1406 & 977 & 881 & 1947 & 2300 & 0 & & & \\
\hline Washington & 229 & 696 & 1096 & 2754 & 2897 & 1165 & 0 & & \\
\hline Denver & 1852 & 1043 & 2126 & 1189 & 1267 & 1314 & 1707 & 0 & \\
\hline Dallas & 1649 & 955 & 1394 & 1431 & 1791 & 509 & 1414 & 805 & 0 \\
\hline Seattle & 3025 & 2184 & 3496 & 1193 & 866 & 2731 & 2880 & 1426 & 2222 \\
\hline
\end{tabular}

Table IV. Mileages between US cities

Figures 10 and 11 are one-dimensional and two-dimensional maps of distances between the given cities in the matrix. Quite simply, an MDS approach attempts to fit all of the given data into a pre-selected number of dimensions, adjusting all of the objects to achieve the lowest level of overall error. 


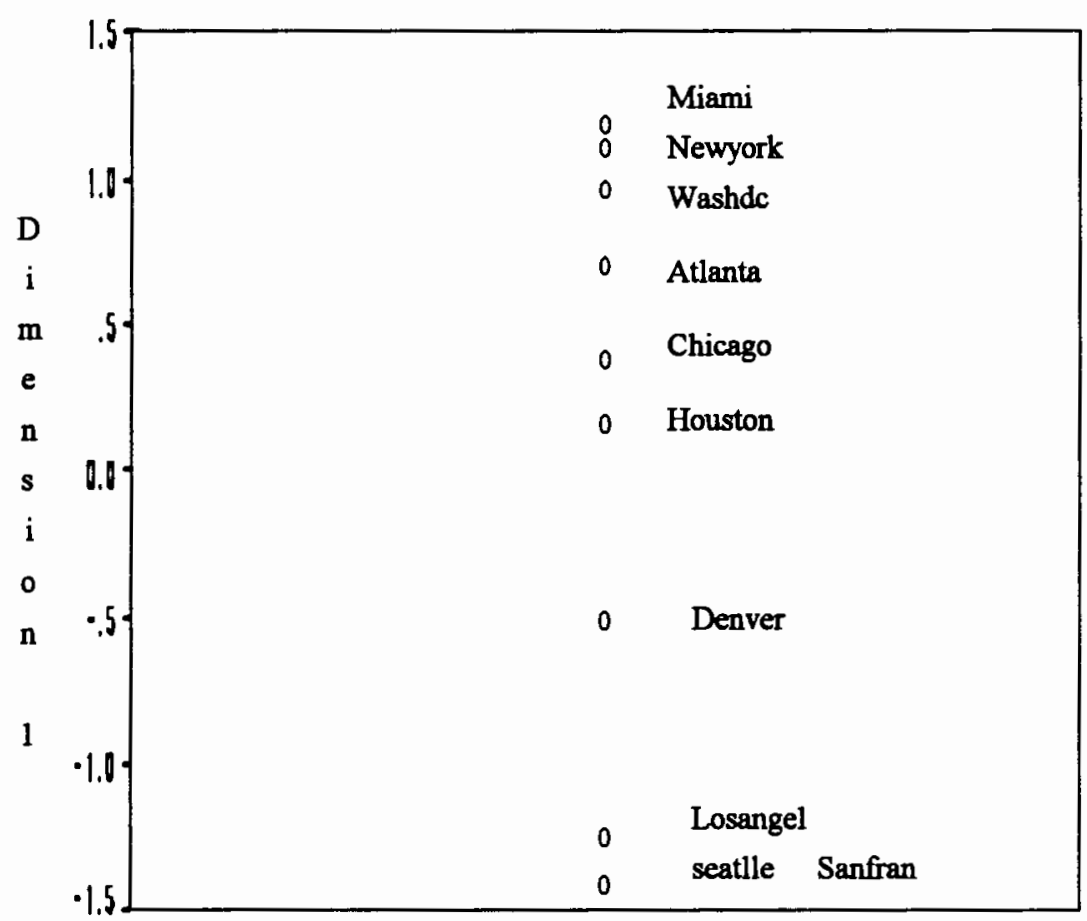

Figure 10. One-dimensional MDS map of city distances

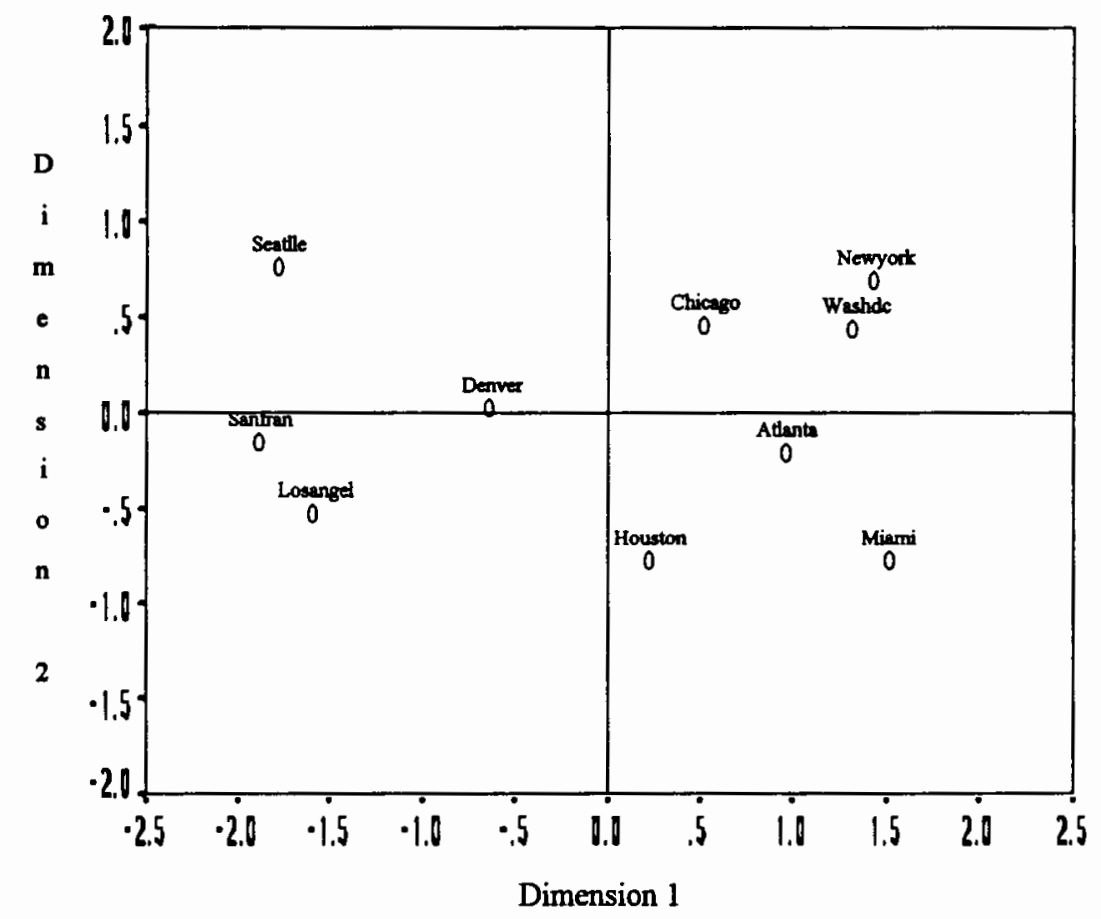

Figure 11. Two-dimensional MDS map of city distances 
Of course, the number of dimensions which is used is crucial to the accuracy of the resulting map. As a result, the one-dimensional map of the cities hardly produces an accurate representation of the relative distances that are given in the matrix. In this idealized example, a two-dimensional map solves the fit problem completely. However, if the original data came from measurements of the distances between those cities as they are actually situated on the curved and uneven surface of the earth, then even a two-dimensional model would contain some error--due to the mismatch between the number of dimensions latent in the data with the number of dimensions used in the mapping process.

Though the above example is adequate for illustration, it is also somewhat limited in describing the actual use of MDS. In the above example the number of dimensions latent in the data was already known beforehand. In contrast, MDS is most useful in those situations for which the number of dimensions is not known. In fact, in many situations MDS is used to infer the number of dimensions. Therefore, in actual use, the procedure often involves obtaining a number of possible solutions and assessing the level of error for each to decide on one preferred configuration.

The procedure is first to obtain a one-dimensional solution, a two-dimensional solution, and so on up to some number of dimensions considered more than sufficient for the input data. The goodness-of-fit measure, termed "stress," is also calculated for each solution.

(Rosenburg \& Sedlak, 1972, p. 243)

The level of error, or stress, in a map is measured as "a normalized sum of squared residuals in which $0 \%$ stress means that a perfect monotone relation exists 
between input and output distances" (Rosenburg \& Sedlak, 1972, p. 243). That is, stress is a measure of how well the resultant MDS map fits the input data. For the above data, a one-dimensional solution had a stress of 19307 and a two-dimensional solution had a stress of .00233 . Kruskal and Wish (1978) suggests that for many purposes a stress of $10 \%$ is fair and one of $5 \%$ is good. Given this guide, the onedimensional solution is obviously unacceptable and the two-dimensional solution is quite accurate.

The relationship between input and output distances can also be represented in a scatter plot (sometimes called a Shepard diagram) of the actual ranked distances in the input data versus the ranked distances in the output map. The scatter plots in figures 12 and 13 are examples of one and two-dimensional solutions for the city data respectively. The high stress in the one-dimensional solution can be seen in how much the plotted scores in Figure 12 vary from a direct linear relationship. On the other hand, the accuracy of the two-dimensional solution is reflected in the tight linear regularity of the scatter plot in Figure 13. However, though they can act as rough guides, absolute measures of stress in themselves are not sufficient to decide which level of dimensionality is correct. 


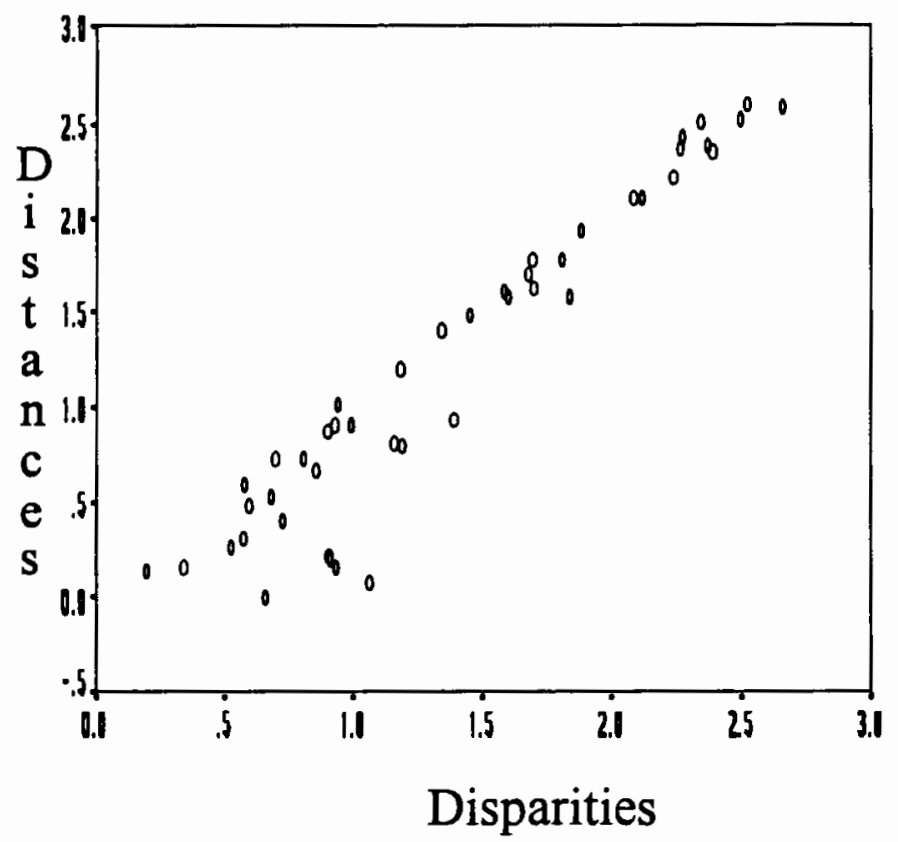

Figure 12. Scatter plot of disparities for the one-dimensional map of city distances

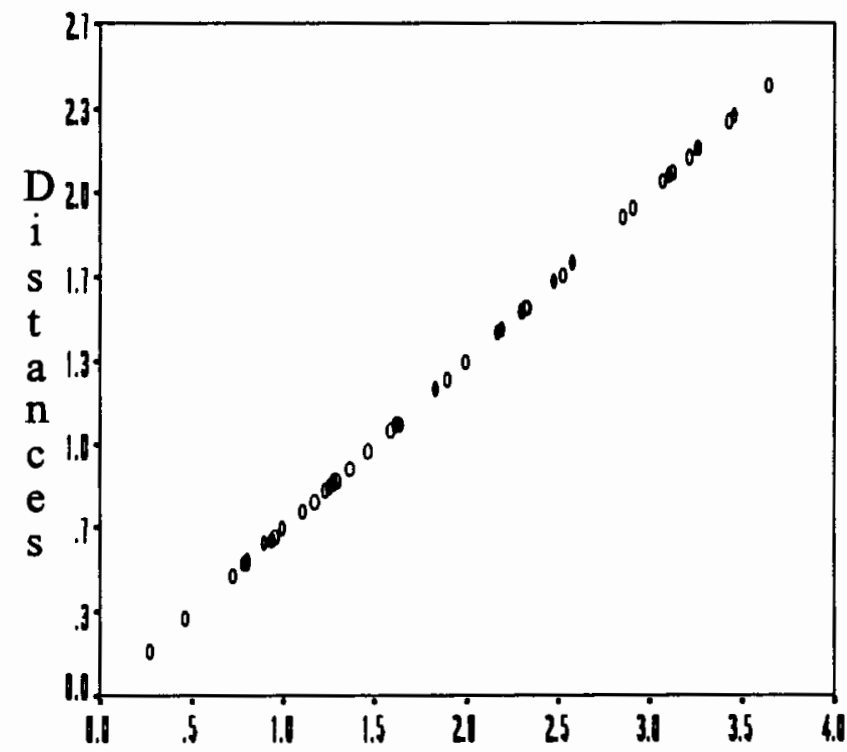

\section{Disparities}

Figure 13. Scatter plot of disparities for the two-dimensional map of city distances 
It is not, unfortunately, possible to say exactly what a "good" or "bad" s-stress value is. We do know however, that the value is a function of many things in addition to the amount of error in the data. For example s-stress gets larger when the number of stimuli or matrices goes up. (Norušis, 1992, p. 183)

Most importantly, an absolute measure of stress does not necessarily solve the problem of which solution among many potentially acceptable ones is the best. The reason for this ambiguity is that, generally, stress tends to decrease with increased dimensions and this is true even as the number of dimensions in the map exceeds the number of dimensions latent in the data (Dillon \& Goldstein, 1984; Hare, et. al., 1992). Consequently, though one reaches more acceptable levels of fit with higher levels of dimensionality, this "does not mean that higher dimensional solutions are necessarily correct in the sense of uncovering the true structure underlying the set of original proximity data" (Dillon \& Goldstein, 1984, p. 118). In other words, there may be more than one possible map which falls within acceptable levels of stress, though some of these solutions may contain superfluous dimensions which will affect the validity and interpretability of those maps. "A trade-off must then be made between the fit of the solution and the number of dimensions" (Hare, et. al., 1992, p. 329)--the goal of which is to achieve "the lowest dimensionality with an acceptable level of fit" (Hare, et. al., 1992, p. 328). One common procedure for resolving the trade-off and determining the appropriate number of dimensions is to examine the improvement in the stress values as the number of dimensions is increase--see Table V and Figure 14. 


\begin{tabular}{|l|c|c|c|}
\hline Number of Dimensions & 1 & 2 & 3 \\
\hline Stress & .19307 & .00233 & .00232 \\
\hline Improvement & N/A & .19074 & .00001 \\
\hline
\end{tabular}

Table V. Stress and improvement of stress by number of dimensions

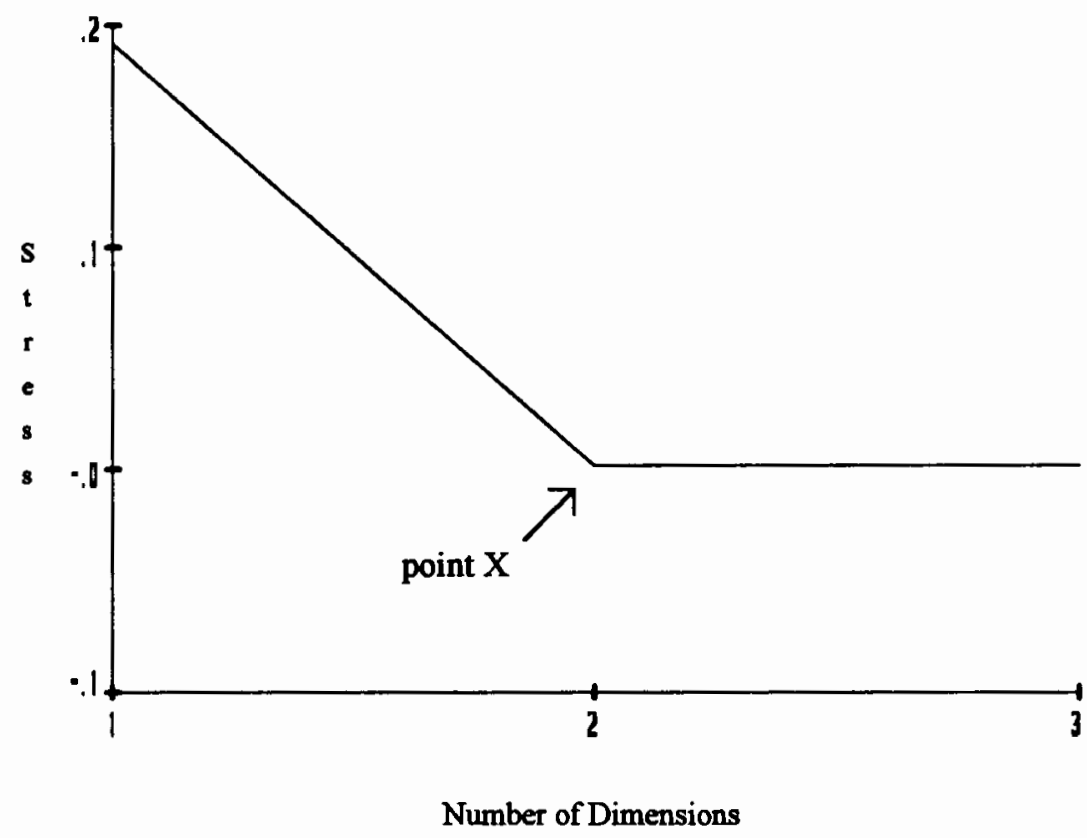

Figure 14. Graph of stress versus the number of dimensions in city distances solutions Given a plot of this relationship the researcher can often identify an 'elbow pattern'--the bottom of which (point $\mathrm{x}$ ) indicates the preferred number of dimensions. That is, the graph is used to reveal that point before which increases in dimensionality resulted in substantial improvements of fit and after which further increases in dimensionality resulted in insignificant improvements of fit. Often, this optimum level of dimensionality is quite obvious and other times it is more difficult to ascertain. The 
degree of certainty one can maintain about a particular judgment of dimensionality depends on both the given distribution of the data as well as the aims of the researcher.

Very often however, considering both the overall stress and the relation of stress to increases in dimensionality can produce a high degree of confidence in the selection of one level of dimensionality as opposed to others. In these cases, the resulting map is then accepted as a valid reflection of the given data and interpretation of the map can begin.

There are two basic aspects of interpretation. First, the relationships between the mapped objects can be significant in themselves. This is especially true when the mapping reveals unexpected configurations. Second, one can assign a meaning to a map's dimensions, thereby attempting to explain the reasons for the data's distribution. That is, one can assign attributes to the dimensions of a map which explain the distribution of the objects within the multidimensional space.

The significance of the relationships between various labels in a map is provided both by the research purpose and the theory being used to propose the research question. One representative example of how the relationships between labels can be significant in themselves can be found in Taylor, Bassili, and Aboud's (1973) use of MDS for the study of ethnic identity. In order to test whether language, culture, or geography was more salient in determining ethnic identification of their Canadian subjects, twelve "stimulus labels were prepared which represented different combinations of these factors" (Taylor, et. al., 1973, p. 170)--e.g., "French speaking 
English Canadian in Quebec." These labels, along with the label MYSELF were then presented to both English Canadians and French Canadians for similarity judgments.

On the assumption that perceived similarity between a label and MYSELF would indicate a salient aspect of identity (Taylor, et. al., 1973), the relationships between the labels were assumed to reveal which of the potential factors of ethnic identification was most salient for each of the two groups. Given these assumptions, it was concluded that:

The configuration of stimulus labels judged to be most similar to MYSELF for both French and English Canadian respondents revealed that language was the most salient dimension of identity. Thus for French speaking Canadians French speaking labels and for English speaking Canadians English speaking labels were clustered very close to MYSELF in the multidimensional space. Of significant, but secondary importance was the cultural background factor. For example, this was evidenced by the fact that for French Canadians a "French speaking English Canadian" was closer to MYSELF than was an "English speaking French Canadian."

(Taylor et al., 1973, p. 170)

Ultimately the distribution of labels within a space should lead to a suggestion of some meaning for the space's dimensions. This is an important step, because the dimensions of a space are assumed to represent dimensions of judgment which were used by the subjects in comparing the objects. Therefore, Dillon and Goldstein suggest that "if the uncovered dimensions cannot be interpreted they most likely do not exist" $(1984$, p. 147) and the results should be suspect.

Two basic approaches for interpretation of a map's dimensions are common. One approach involves relating additional statistical data to the particular configuration revealed by the mapping and the other uses the researchers' previous knowledge to 
inform the interpretation of the space in which the data is distributed. The former is often referred to as an objective approach. The latter is often referred to as a subjective approach. Though I chose a subjective approach for the present study for a detailed explanation for this choice), I will discuss both approaches in some detail. I will maintain this level of detail for two reasons. First, even though I did not choose an objective approach, the principles of interpretation for the two approaches are similar. Further, for some readers, it may be easier to grasp the process using one or the other description Second, fully understanding any methodological choice involves understanding the alternatives. I will describe both in some detail even though I chose to use the latter.

Common procedures within an objective approach are property fitting and canonical correlation analysis. Property fitting uses an a priori salient attribute to reveal a meaningful directional vector within the map of stimulus objects. A directional vector within the stimulus space is a line that represents an increase in a specified attribute. A directional vector may be used to assign meaning to a mapped dimension in the following manner. If, by independent means it is known that (1) the objects used for MDS show an ordered increase in a chosen attribute (or perceived attribute) and that (2) the objects are aligned along a directional vector within the stimulus space in a manner that is consistent with the a priori rankings of attribute strengths, then it can be inferred that the directional vector within that space represents an increase in the attribute generally. This method can be especially easy when all of the objects either 
possess or do not possess the specified attributes (e.g., when all the objects are either diet or non diet drinks). However, meeting these conditions doesn't automatically result in a valid interpretation for the given data can show a similar, even exact, ranking along more than one attribute. To address this potential ambiguity canonical correlation analysis simultaneously determines the possible relationships of a number of related attributes to the perceptual objects within a map. In this way, the strongest attributes, among a number of possibly similar or related attributes, are selected as the most salient for the map's interpretation.

Though property fitting and canonical correlation analysis do offer the advantage of being more quantitative, one should note that quantification doesn't imply greater objectivity or validity: the researcher is still responsible for selecting the most relevant attributes and there is always the chance that the most salient attributes for interpretation will be left out. These potential limitations are of particular interest because often the most salient attributes aren't apparent until one actually examines the resulting map.

A more openly subjective method "relies solely on the position of the objects in the stimulus space" (Dillon \& Goldstein, 1984, p. 147) and the researcher's background knowledge. That is, often the objects at the extreme edges of the stimulus space suggest attributes that can be used to explain their extreme opposition within that space. Many times these attributes can be used to explain the positions of the objects in general and assign directional vectors. However, this method is most useful in 
situations when the judgments are not obviously discrete. In these more exploratory studies a reliance on the researcher's past experience and judgment can also allow the attributes to emerge solely from the resulting distribution of the data rather than a preselected list of attributes.

Aboud and Christian's (1979) study of ethnic identity development in grade schoolers in Canada offers a good example of this approach. The study compared ethnic categorizations and self-identifications of second and fourth grade students from four different ethnic groups. Because of the comparative and exploratory nature of the study, the dimensions of judgment could not be pre-selected. In fact, one of the research questions was whether, and to what extent, the different groups of subjects used a consistent set of criteria for making self-other comparisons. Therefore, having chosen an attribute-free approach, Aboud and Christian used a subjective interpretation procedure and offered interpretations of the dimensions in the resulting maps solely on the basis of object distribution. For example, one two-dimensional map of fourth grade Greek students' judgments showed "French," "Greek," and "English" clustered together on one end of the map and "Chinese" and "Indian Canadian" clustered together on the other end of the map. From this they concluded that the "ordering was clearly one based on physical appearance" (Aboud \& Christian, 1979, p. 185) with all white persons judged as being similar to each other and different from all non-white persons. Similarly, the second dimension of the same map showed an ordering which corresponded to the demographics of the school, with all groups which were not 
represented in the school placed toward one edge of the map. Therefore, the authors suggested that the second dimension "was defined in terms of familiarity" (Aboud \& Christian, 1979, p. 185). A similar procedure of relating object distributions to researcher knowledge of the objects was followed with the other maps.

\subsection{Cluster Analysis}

Many of the basic principles of cluster analysis are similar to those of MDS. Cluster analysis also attempts to graphically represent the pattern of similarities between various objects. Both the range of objects and the basic measures which can be used for each procedure are similar. In the case of this study, the objects were labels related to ethnic and linguistic identity and the measure of similarity was a set of frequency counts of co-occurrence obtained from a card-sort task. However, the procedural and interpretive goals of the different procedures are somewhat different. The goal of an MDS procedure is a spatial configuration which best represents the sum of the relationships between the objects in question. The interpretation of an MDS space assumes that the orderings of the objects in the space are meaningfully related to identifiable and interpretable attributes which explain the configuration. In contrast, "cluster analysis seeks to divide a set of objects into a small number of relatively homogenous groups on the basis of their similarity" (Bailey, 1975, p. 59). "In agglomerative hierarchical clustering, clusters are formed by grouping cases into bigger and bigger clusters until all cases are members of a single cluster" (Norusis, 1992, 
p. 85). The result of this process is a tree structure, or dendogram, in which the closest objects, or set of objects, are linked together, then the next closest object, or set of objects, are linked, etc., until all objects are linked together in a single group. In this tree, if two objects appear linked together as a unique group, they are interpreted as being more similar to each other than to all other objects in the analysis. Procedurely, "the basic goal is to maximize homogeneity (minimize variation) within each cluster ... [and maximize] variation between clusters ..." (Bailey, 1975, p. 61). The final goal is to identify a level of agglomeration which represents a set of basic clusters which are both exhaustive and mutually exclusive (Bailey, 1975). Unlike other techniques for examining clustering (e.g., discriminant analysis, $\mathrm{K}$ means clustering, etc.,), in hierarchical clustering, "group membership for all cases is unknown. In fact even the number of groups is unknown. The goal of [the] cluster analysis is to identify homogeneous groups or clusters" (Norušis, 1992, p. 83; my emphasis). The following simple example from Norušis (1992) provides a good demonstration of the basic principles of a hierarchical cluster analysis. The tree structure in Figure 15 is an example of an agglomerative cluster analysis of twenty beers based on measures of level of alcohol, level of sodium, caloric content, and cost. The purpose of the analysis was to identify a basic set of meaningful clusters of beers. After being standardized, the combined differences between each pair of beers were transformed into Euclidean distances in much the same way as would be done for an MDS analysis. ${ }^{37}$ These distances were then re-scaled into a 0 to 25 scale and the beers were agglomeratively 
clustered. The resulting tree structure shows both the order of the clustering and the distances at which various clusters were made. Using this tree structure, a level of clustering was identified which represented the minimum distance at which the clustering was meaningful. The clusters were then labeled in reference to the shared characteristic(s) of the clusters' members.

Rescaled Distance Cluster Combine

C A S $\mathbf{E}$

Label

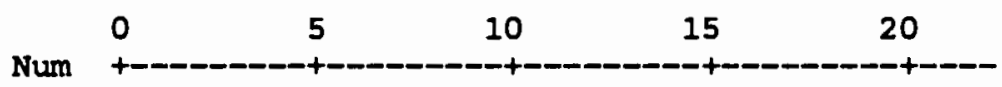

Coors

Hamms

Budweiser

Lowenbrau

Schlitz

Strohs Bohemian

Hellmans old style

old Milwaukie

Auggberger

Miller lite

Schlitz light

Budweiser lite

Coors light

Michelob light

Heineken

Kirin

Kronenbourg

Becks

Pabat extra light

olympia gold light

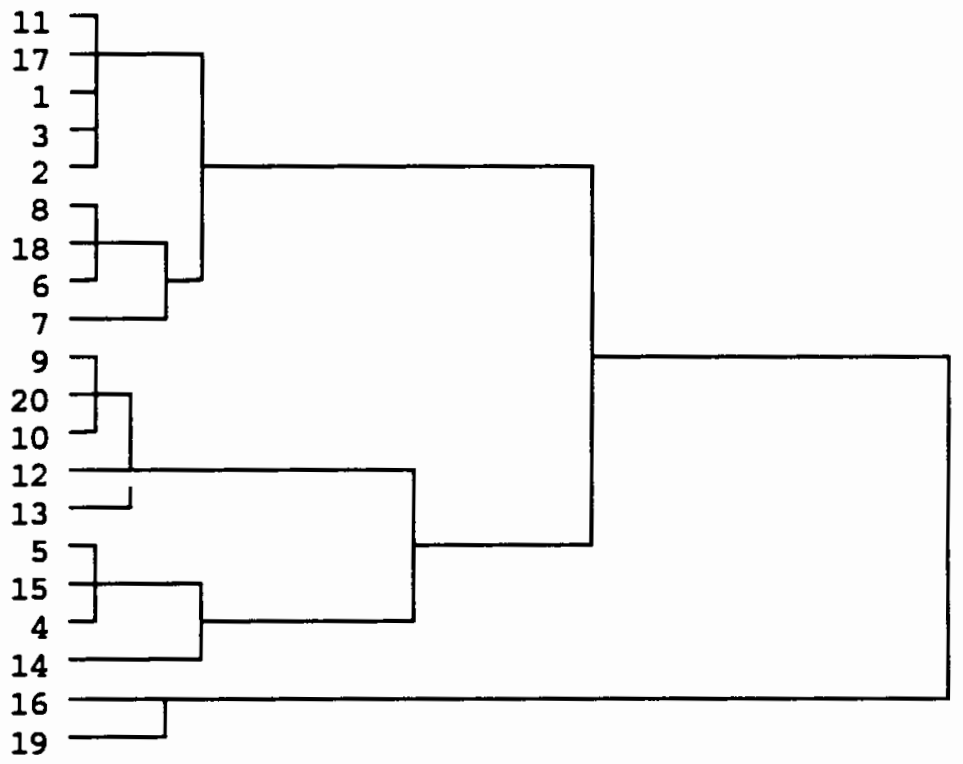

Figure 15. Sample dendogram: Results of hierarchical cluster analysis of beer data

Looking at the dendogram, it appears that the five cluster solution (very light beers, light beers, imported beers, high sodium beers, and "average" beers) may be appropriate since it is easily interpretable and occurs before the distances at which clusters are combined become too large.

(Norušis, 1992, p. 93). 
The results of a hierarchical clustering are often superimposed onto an MDS map, as in the following map containing characteristics of a good teacher (Kono, 1995). In this map, the basic clusters in the tree structure are represented by drawing boundaries around each cluster's members.

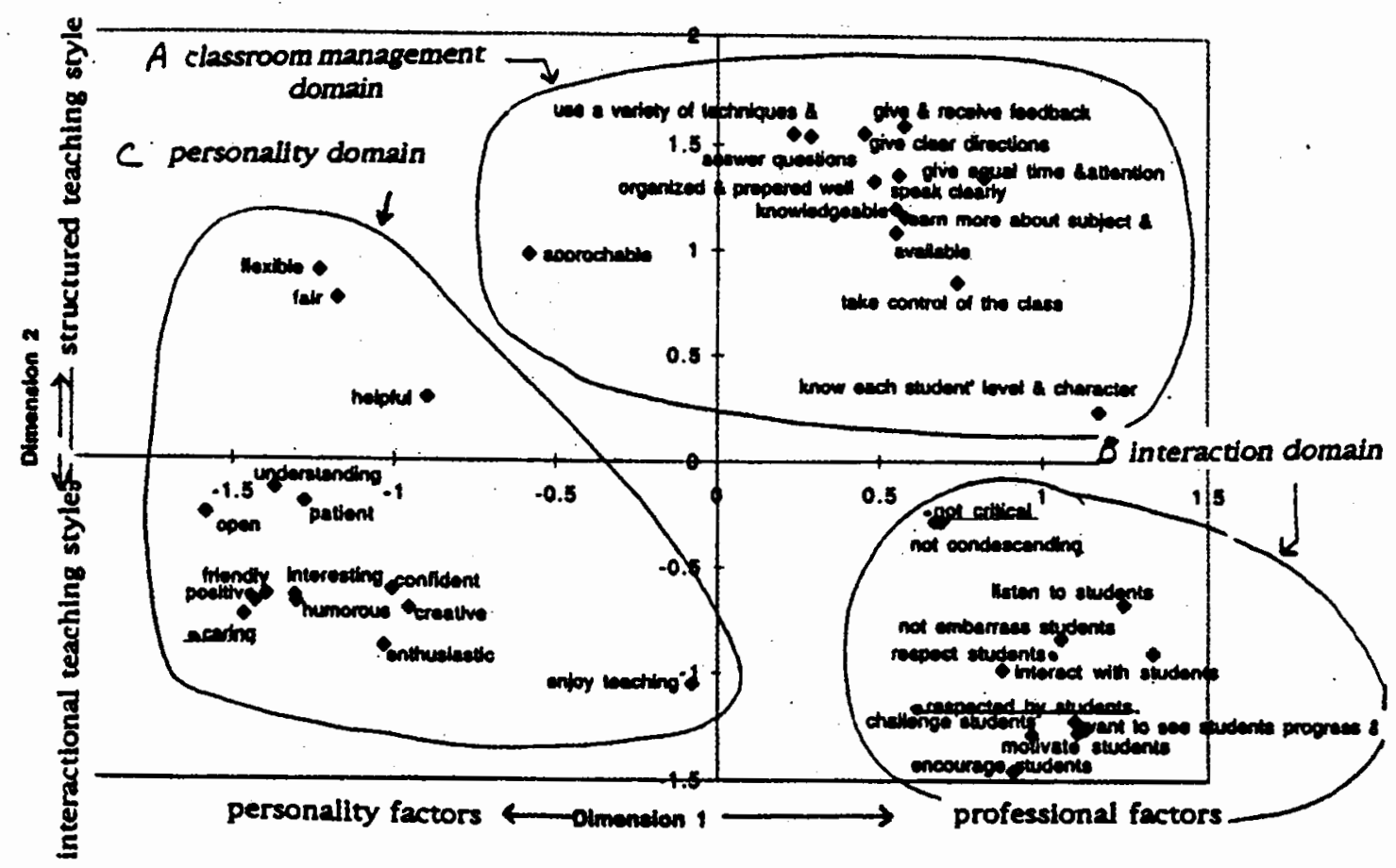

Figure 16. Sample MDS map: The concept of "a good teacher"

However, even though MDS and cluster analysis are often combined, the interpretation of the two procedures should not be confused. Two objects can appear very close together in an MDS space and yet be assigned to different clusters. Further, an object can appear on one extreme end of an MDS space and yet be clustered with objects on the opposite end of that space. If the procedures produce such different 
results, then it might be reasonable to ask why the results of the two procedures are often combined.

Kruskal and Wish (1978) have offered the conceptual metaphor of neighborhoods for understanding the how cluster analysis is related to the MDS space. Extending this metaphor, Blunk (1994) liken the difference between MDS and cluster analysis to that between neighborhood of residence and family affiliation. Even though two items both live in the same neighborhood they can also have primary family affiliations to very different groups living in other neighborhoods. For example, in Figure 16 "approachable" appears in the upper left hand quadrant, closest to the characteristics "flexible," "fair" and "helpful." However, "approachable" is actually clustered with the objects in the upper right hand quadrant and "flexible, "fair," and "helpful" are clustered with items in the lower left hand quadrant.

This difference is more than just a conceptual one. Combining both levels of information has a practical effect on the process of interpreting a map. If one had only the results of the cluster analysis alone, it would not necessarily reveal the underlying dimensions represented in the above MDS map. On the other hand, the MDS results alone might appear to have confusing anomalies which would have made identification of the dimensions more difficult. For example, why do the labels "flexible," "fair," "helpful," and "approachable" appear in the upper left quadrant? They could seem to be personality characteristics, but why are they separated from the other personality related labels in the lower left quadrant? When the cluster analysis is combined with the 
MDS map, the interpretation becomes clearer. First, the relative placement of the clusters is consistent with the given interpretation of the dimensions. Understandably, the classroom management cluster appears in the structured / professional quadrant, while the personality cluster appears in the personality / interactional quadrant. Second, the ordering of individual items within each cluster is also consistent with the interpretation of the dimensions. For example, when one compares "giving clear directions" and "approachable," it is reasonable that being "approachable" is more related to personality characteristics and that "giving clear directions" is more related to professional concerns. Similarly, when one compares "caring," and "fair," it is reasonable that being "fair" is more directly related to concerns about formal teaching issues and that "caring" is more directly related to issues of student / teacher interaction. Now, this is not to say that these classifications are exclusive. One could rightly hold that fairness is also related to interaction; however, the primary issue in the MDS space is the relative ordering of items along a continuum of more or less rather than exclusive membership. Finding meaning in the orderings along a dimension is the primary concern of MDS interpretation. The issue of assigning exclusive membership is a goal addressed by cluster analysis. In this example, the dimensions of the map are reflected in the ordering of the clusters and the ordering of individual items within each cluster. Further, apparent anomalies in the MDS map are explained through the additional information provided by the cluster analysis. In short, when combined together, the interpretation of the map becomes both clearer and richer. 


\subsection{Application of MDS to the Question of Vietnamese Ethnic and}

\section{Language Identities.}

Dillon and Goldstein (1984) have presented a four-step decision framework for MDS consisting of the following:

1. Identification of all relevant objects to be evaluated.

2. Selection of an attribute based or an attribute-free approach.

3. Deciding on the appropriate scaling technique for the selected approach.

4. Interpretation of the results.

Since these decisions are common to all MDS applications, I used Dillard and Goldstein's framework to address the particular decisions relevant for this study.

\subsubsection{Identification of All Relevant Objects to be Evaluated}

In the question under study, the objects to be evaluated were self-descriptions which represented potential identity schemata related to the concepts of ethnicity and language for the Vietnamese-American subjects. Therefore, the first step was to generate an over-inclusive set of stimulus labels (Taylor, 1975) which were relevant to the subjects' ethnic and language identities. This list was generated in two stages. First, a large set of over 1300 self-descriptions was collected from 43 subjects through three modified versions of the Twenty Statements Test (Khun and McPartland, 1954): TST1 was open-ended; TST2 focused on ethno-cultural identity; and TST3 focused on language. Second, the descriptions were reduced to a final list through two data reduction strategies. The first of these strategies involved grouping the descriptions into categories. The second strategy involved considering only those categories that 
occurred in the results of more than one version of the TST. These two issues--the collection of relevant self-descriptions and data reduction--will be described in detail later in this section.

The large degree of variability in the area of ethnic identity, discussed previously, led to the present empirical questioning of the relationship between the ethnic and language identities of Vietnamese-Americans. Since the purpose of using MDS was to allow the actual relationships between identity labels to emerge naturally, it was important that (1) the initial range of labels related to the ethnic and language identities of the subjects not be predetermined and (2) that the labels themselves reflect as little researcher bias as possible. These considerations required that the labels be produced by the subject community itself.

Within the study of ethnic identity, Taylor, Bassili and Aboud (1972; 1973), Giles, Taylor, and Bourhis (1974), and Giles, Taylor, Lambert, and Albert, (1975) used extensive informal interviews and conversations within their subject communities to identify possible dimensions of identification and then used these possible dimensions to generate labels which would test the salience of each possible dimension. One advantage of this procedure was its attempt to tap the emic meanings that these descriptions had for the various subject communities. However, this presented a two practical problems. First, any researcher using this approach would have to spend a large amount of time within these communities in order to minimize bias. Second, 
since the actual level of bias in such situations is generally dependent on the quality of the relationship between the researcher and the subjects, it is not verifiable.

The Twenty Statements Test (TST) designed by Khun and McPartland (1954) offers a more economical way to gather large numbers and types of identity labels. Consequently, the TST has been used by many researchers to collect data for the study of personality (Khun \& McPartland, 1954), aspects of identity (Hoelter, 1985), and ethnic identification (Verkuyten, 1992). The TST asks subjects to list in writing up to 20 possible answers to the open-ended question "Who are You?" The answers may be one word, a phrase, or a single sentence. Besides being both economical and minimally intrusive, the TST had the advantage of being consistent with both the phenomenological and multidimensional assumptions of an information-processing approach to the study of identity. The identity descriptions were produced by the subject community itself and the subjects could answer the question in any way they pleased.

However, initial trials revealed two difficulties with the TST. First, faced with the de-contextualized task of the original TST, the subjects tended to give meaning to the task by explicitly understanding it as a "data collection activity" in which the researcher probably had specific goals which the subjects' responses would fulfill. This result was consistent with difficulties which Markus and Kitayama (1992) found in cross-cultural uses of the TST. As a result, subjects often felt lost about what type of 
response to give and often tried to predict my goals for administering it. They often asked me questions such as:

"What kind of answer are you looking for?"

"What do you want?"

"What kind of thing am I supposed to say?"

Second, the de-contextualized TST tended to produce descriptions which emphasized personal and interpersonal descriptions, rather than descriptions which reflected social group memberships.

Therefore, I experimented with various contexts which were more natural for the task while at the same time being open-ended enough to allow for any type of subject response. The solution came in a fictitious situation in which the subjects were to imagine planning a first letter to a new pen pal whom they as yet knew nothing about. In preparing for their first letter they were to use the TST format to brainstorm self-descriptions--some of which they might use in the fictitious letter and some which they might not use.

The modified TST also solved the second problem of the de-contextualized TST by producing a wider range of descriptions, representing different dimensions of identity, many of which were social-group oriented. However, a single open-ended TST was not sufficient to produce the actual set of labels which was used for the scaling process. Instead, three versions of the TST were given consecutively. TST1 consisted of an open-ended version. TST2 focused specifically on those things which were important for describing the subject ethnically and culturally. Lastly, TST3 asked 
for a list of those languages which the subject felt was important for describing who he/she was. All three versions contributed to the list of descriptions used for the cardsort procedure. The reasons for this decision and the method for combining the results of the three TSTs are as follows.

Given an open-ended TST, I expected that the subjects would produce a range of identity labels. On the one hand, I expected that subjects would probably produce some labels which were clearly relevant to ethnic or cultural identity (e.g., Vietnamese, Asian, etc.,). On the other hand, I expected that subjects would probably produce a number of labels which were clearly not relevant to ethnic or cultural identity (e.g., sister, student, etc.,). I also expected some labels would have most probably fallen within a difficult gray area of relevance (e.g., I use chopsticks, I respect my parents, I love basketball, etc.).). Since including superfluous labels which were clearly not relevant to the issues of ethnic identity or language choice would have adversely affected the validity of the scaling results, the TST results had to be reduced to that set which were most salient to the identity issues under study. Further, there would have been methodological difficulties with relying solely on a single open-ended TST. In that case, which particular descriptions were culturally relevant and which were not would have been my own personal decision. However, how could I have decided this issue? Further, what would have assured the validity of my choices?

Another way I could have focused the TST on the issues in which I was interested would have been to directly ask for those self-descriptions which from the 
subject's own perspective were cultural or linguistic. TSTs 2 and 3 did exactly this. However, using only focused TSTs would have also had serious limitations. The focus of the very questions would have most probably produced many descriptions which were not necessarily valid parts of a subject's identity. For example, it could be the case that someone for whom race is not ever an identity issue readily produces racial self-descriptions when asked to do so directly.

The problem was to achieve some form of valid data reduction which was also consistent with the phenomenological principles behind both the use of the TST in general and the attribute-free MDS methods to follow. The solution was to administer both unfocused and focused TSTs and then take the intersection of the three versions. That is, only if a description appeared on more than one TST, was it included in the final list of potential descriptions.

Since the purpose of the TSTs was to produce an over-inclusive list, I decided to determine the intersection of labels on a group level. That is, all the group's descriptions for each TST were aggregated before carrying out the data reduction steps. This was done in order to balance the goals of data reduction versus overinclusiveness. Either an excessively inclusive or an excessively restrictive reduction procedure would have been undesirable. Therefore, the most restrictive choice of first taking the intersection of each individual's TST data and then aggregating and categorizing the results was rejected. Instead, I chose the more inclusive process of first aggregating the results of each administration of the TST and then identifying the 
descriptions which were common to both lists of aggregated descriptions. The purpose of these procedures was to produce a set of descriptions which reasonably represent the range of cultural and linguistic identity issues for the group as a whole. This approach relied on the following assumptions:

1. If a description appeared on TST 1 , then I could be reasonably sure that it was probably relevant to personal identity.

2. If a description occurred on both TST 1 and 2 , then I could be reasonably sure that it was related to cultural identity.

3. If a particular language was mentioned on TST1 and 3, I could be reasonably sure that it was related to personal identity.

4. If a particular language appeared on TST2 and 3, I could be reasonably sure that it was culturally relevant.

5. If a particular language appeared on all three TSTs, I could be reasonably sure that it was relevant to cultural identity.

Therefore, each subject was given an open-ended TST, a TST which asked for those self-descriptions which were relevant to their ethnic or cultural identities and a TST which asked for those languages which the subject felt was important for understanding who he/she was.

Initially, the aggregated results of each TST were placed into separate coded data tables. Since 9 of the subjects completed either part or all of their TSTs in Vietnamese, translations had to be done. Any descriptions which were written in Vietnamese were directly translated into English. The translation was done by a group of three bilingual Vietnamese and English speakers. All of the translators were born in Vietnam and have lived in the US between ten and eighteen years. One of the group 
was a working court and medical translator. Any disagreements on a translation were discussed among the group until consensus was achieved. Originals and translations appeared together within the data tables. Back translation was not necessary at this stage since I knew that many of the translated items probably were not going to make it through the data reduction procedure. Further, even those items that would continue to be considered were important only in so far as they were members of a group of items. As long as the translation procedure did not significantly affect their final group memberships, the subtleties of various versions were not important.

Once all the descriptions were entered into their respective tables, I began a semantic grouping procedure. The goal of the procedure was to sort the data into groups by looking for descriptions which were similar in meaning. At the same time, I also looked for groups of descriptions which appeared in more than one TST. I made the decision to simultaneously group within and across different versions because this was the most efficient and focused method. Often, when a semantic grouping procedure involves more than one source of statements, each list is grouped independently and then the resulting groups for each set are compared for similarities and differences. Since I was only concerned with those groups which were similar for the various TSTs, I had no need to find groups that were unique to each list of descriptions. Therefore, I chose not to independently categorize each TST before they were compared. Instead, at each stage, I simultaneously considered the categorization of all the data in all the TSTs. I will say more on this shortly. 
The basic grouping procedure consisted of three stages. In the first stage, I looked at a single TST's set of descriptions for exact and close matches. Descriptions that were exact matches had to be precisely the same in all respects (e.g., "Vietnamese" and "Vietnamese"). Close matches consisted of descriptions which contained the same phrases fulfilling the same syntactic and pragmatic roles in each of the descriptions (e.g. "I am Chinese," "I consider myself as Chinese" and "I think I am very Chinese"). Each category was then individually searched for a variety of negations (e.g., "Vietnamese" vs. "not Vietnamese"; "I will always be Vietnamese" vs. "I will never be Vietnamese") and the negations were assigned to a separate category of their own. During the second stage, I grouped the remaining descriptions into categories expressing common themes (e.g., "Comments on education," "Comments on family," etc.,). In the third stage, these thematic categories were then individually subdivided into categories of common concern (e.g., Comments on family: comments about the importance of order and hierarchy within the family, comments on the importance of obeying parents, etc.,).

As I mentioned above, I did not thoroughly categorize each list independently. Usually, as soon as I found several "matches" or several descriptions expressing a common theme in the results of one TST, I looked for at least one matching or similar item in another TST. If a category of descriptions met these criteria, I continued to consider it. For example, as soon as I found several exact and close matches for "Vietnamese" in TST 1, I looked to see if that item appeared in TST 2. Similarly when 
several comments about the theme of family were found in TST 1, I looked to see if TST 2 contained similar items. If no matching items were found, then I ceased to consider the category. In this way, relevant categories were built first and irrelevant categories were simply ignored. I also wanted to avoid including categories which were merely the result of one subject's data. Therefore, once the descriptions from each TST were grouped together into matching sets of categories, I applied an additional criterion. For each TST, I tabulated the number of descriptions in each category that were produced by different subjects. I kept only those categories which occurred in the results of more than one TST and were produced by different subjects in the different TSTs.

The final result of this grouping was an initial set of categories containing an over-inclusive range of self-descriptions relevant to the ethnic, cultural, and linguistic identities of the group as a whole.

Each category of descriptions was given a summary label. Following previous studies of identity which used MDS (Giles, Taylor, and Bourhis 1974; Giles, Taylor, Lambert, and Albert, 1975; Taylor, Bassili and Aboud, 1972; 1973), the label "MYSELF" was added to the list and each label was randomly assigned an identification number. The summary labels were then translated into Vietnamese using a back translation method (Brislin, 1970 ), and the labels and translations were printed onto "3X2" cards. The English and Vietnamese versions of each label appeared on opposite sides of each card. The identification numbers were also printed in the top 
right corner of each side of each card. These cards were used in the free-sort task described below.

\subsubsection{Selection of an Attribute based or an Attribute-free Approach}

The nature of the research question required that (1) the relationships between the labels to be compared should not be predetermined within the comparison process and (2) that the mode of comparison between labels be left to each subject. Therefore, I chose an attribute-free approach as the most appropriate. An attribute-free approach allows the subjects to compare stimulus labels on the basis of their own overall perception of the labels, rather than some set of discrete criteria specified by the researcher.

Two basic procedures were available for obtaining attribute-free comparison data. First, subjects could have performed a direct comparison of each possible stimulus pair. Second, subjects could have sorted labels into groups based on their perceived similarity. There was one compelling reason to reject a direct comparison approach. Considering the large number of labels which were expected to be used in the study, it would have been necessary for subjects to judge an unreasonably large number of pairs. This tended to make direct comparison a poor choice. Therefore, a better alternative for the proposed study was a subjective clustering approach (often referred to as a "card sort"). In this procedure each label was written on a $3 \times 2$ card and subjects were asked to sort the cards into groups based on perceived similarities in the meanings of the labels. 
Subjective clustering offered two advantages. First, it required a more reasonable commitment of time and effort from each subject. Second, it could easily be administered to groups, making it a very economical use of my time. In a trial study, a set of cards was handed to each subject and all of the subjects were asked to sort the cards into piles based on their perceptions of the labels' similarities. However, I found that merely having subjects sort the labels on the basis of similarity often resulted in irrelevant results. When subjects were allowed to sort the labels using any criteria of similarity they wished, many tended to sort the labels based on simple linguistic criteria. That is, some subjects tended to place all cards referring to national identity, languages, racial identity, etc., in respective groups. This simple strategy of grouping, produced data which was not even remotely relevant to the purpose of my study. Therefore the presentation of the task had to be done in a way that would prompt the subjects to avoid this strategy. Previous studies on ethnicity which used MDS (e.g., Aboud \& Christian, 1979; Giles, Taylor, \& Bourhis, 1973; Taylor, Bassili, \& Aboud, 1973) avoided this problem by constructing labels which described various hypothetical people: e.g., an "English Speaking French Canadian Living in Quebec" versus a "French Speaking English Canadian Living in Quebec." Similarly, in their study of implicit personality theory, Rosenburg and Sedlak (1972) avoided this potential problem in a card-sort method by asking the subjects to think of each group of sorted labels as representing a particular person. I chose to let subjects begin the sorting task and then after a few minutes add the instructions to think of each pile as "like a person" 
or "like a kind of person." Subjects were free to use any number of piles they wished and to use a MISCELLANEOUS pile for those cards which they felt did not seem to fit anywhere else.

There were also several important differences between a typical attribute-free card sort and the actual procedure that was used in this study. First, the TST stage of the study produced 45 different labels. Forty-five labels was beyond the maximum number which subjects could easily sort. Therefore, the task was divided into two stages. In the first stage the subjects were given 24 basic labels and were asked to sort them into any number of categories. The first 24 labels were selected because they most directly addressed the research questions. These labels represented different identities of people, different cultures, different languages, and different values. In the second stage, the subjects were given 21 additional labels (mainly consisting of personal and group attributes) and were asked to fit them into the categories which were produced during the first stage of the sorting. During this second stage, subjects were not allowed to change or add to the categories which were present. By breaking the sorting process into two stages, I was able to use all 45 labels without demanding too much from the subjects.

Second, unlike a traditional card sort, subjects were given the chance to have multiple copies of each card and sort the same label into more than one group. A traditional card sort forces the subjects to place each description in only one pile. However, this may force the subjects to make distinctions which do not actually exist 
within their psychological and social lives, and tend to make real distinctions much more defined than they actually are. The validity of this choice seems readily apparent when one considers the issue of multilingualism. If one is forced to sort languages and cultures into unique groups this would, not surprisingly, produce a view of language and culture which is bifurcated and unique. However, as pointed out in section 1.0, whether the relationship between two specific languages and two specific cultures is actually a bifurcated one is an empirical question. Further, this may be true of more than just language and culture. The same could be true of everything from food ways to value orientations. Therefore, the choice to allow multiple categorizations seemed most consistent with the possibility of subjects having multiple group memberships and also with the possibility of different groups having overlapping characteristics. Therefore, in each stage of sorting, subjects were given a deck of cards containing one copy of each card and an plastic holder containing 15 copies of each card. These additional copies could easily be removed from the holder and included in the sorting procedure.

In between the first and second stages of sorting, the subjects were given two additional tasks. First, each subject was asked to label his/her piles of cards. Though I did not make the assumption that the underlying criteria of judgment revealed in the mapping must have matched with the subjects' conscious ideas about their judgments, I asked for group labels as an additional source of information which could help inform 
the interpretation of the MDS results. In addition, each subject was also given the following list of sentences:

1. How I am with my parents

2. How I am with my brothers and sisters

3. How I am with my friends

4. How I am at school

5. How I am at work

Each sentence contained a specific domain which was mentioned in the TST results at least twice. Subjects were asked to consider each sentence in turn and to identify the five labels that they felt were most relevant for each domain. Whereas the MDS results provided a map of relatively de-contextualized identity relations, the ranked frequencies of choices for each domain were used in conjunction with the MDS analysis to explore which dimensions of identity were most salient within the context of specific domains.

The card-sort data for each subject were then used to produce separate matrices of co-occurrence ${ }^{38}$. The matrices consisted of binary entries: if the paired labels cooccurred in the same grouping, a one was entered in the appropriate cell of the cooccurrence matrix; if the paired labels did not co-occur, a zero was entered. This process produced a matrix of similarity measures in which a larger value was given to all co-occurring items than was given to all non-occurring items. These co-occurrence matrices were aggregated and used as the input for the scaling procedure.

\subsubsection{Deciding on the Appropriate Scaling Technique}

One apparent disadvantage of using a subjective clustering procedure was the nature of the resulting data in relation to the output of the scaling procedure. The 
result of each individual card sort was a matrix in which co-occurrences were simply given a larger value than items which did not co-occur. The actual values had no absolute meaning. They merely represented relative orderings of (dis)similarity. In short, they were ordinal rather than interval-level data. However, the mapping procedure seems to produce metric-level (either ratio or interval) output. Typically, this would not be appropriate. Because of the nature of MDS, however, this initial incompatibility is more apparent than real. Empirical tests comparing interval and ordinal-level mappings of the same data have demonstrated that the two levels of data produce remarkably similar results. For example, Shepherd (1966) found that the reconstruction of a known configuration from only the rank orderings of the distances involved is nearly perfect with the use of only 15 points and often reasonably close using fewer points. "The reason for this, roughly speaking, is that even the rank order of distances contain enough metric information under most conditions to recover the original [i.e., metric] configuration" (Rosenburg \& Sedlak, 1972, p. 239). Therefore, the correspondence between input and output in a non-metric approach need not be restricted to a best-fitting linear function, but can be any monotone relation" (Rosenberg \& Sedlak, 1972, p. 239)--i.e., any approximately linear function which is order-preserving. The original example of distances between cities offers a good medium for illustrating this point. Figures 17 and 18 show the results of a nonmetric MDS analysis of the data in Table IV after it had been transformed into ordinallevel data. 


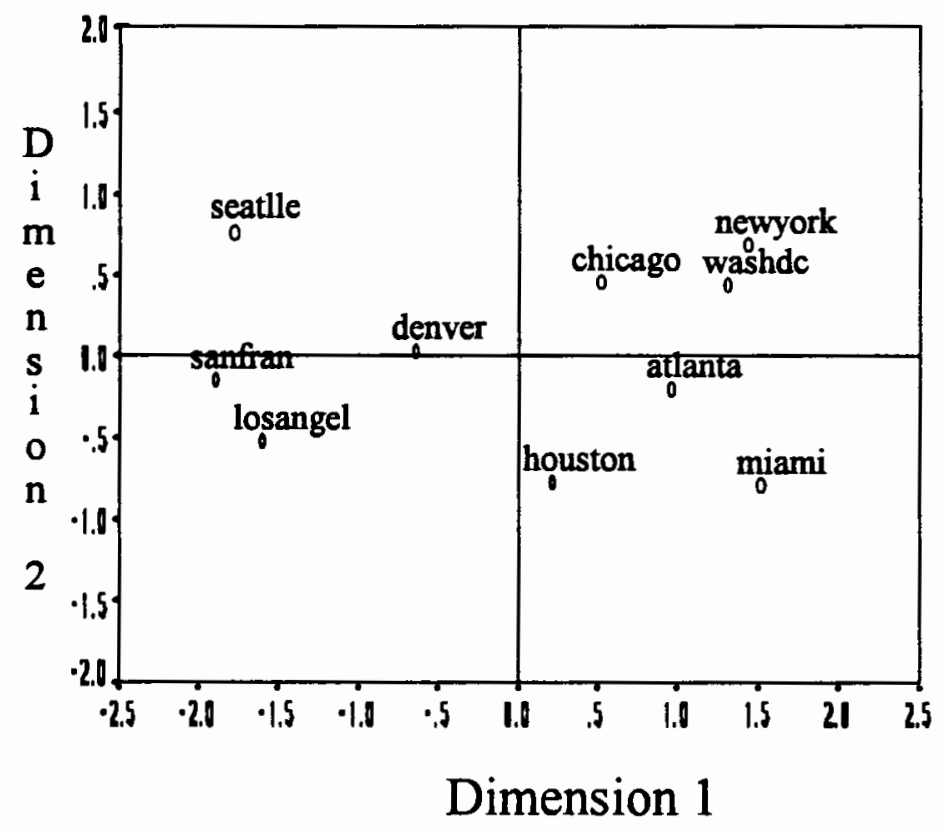

Figure 17. Two-dimensional map of city data: ordinal-level scaling

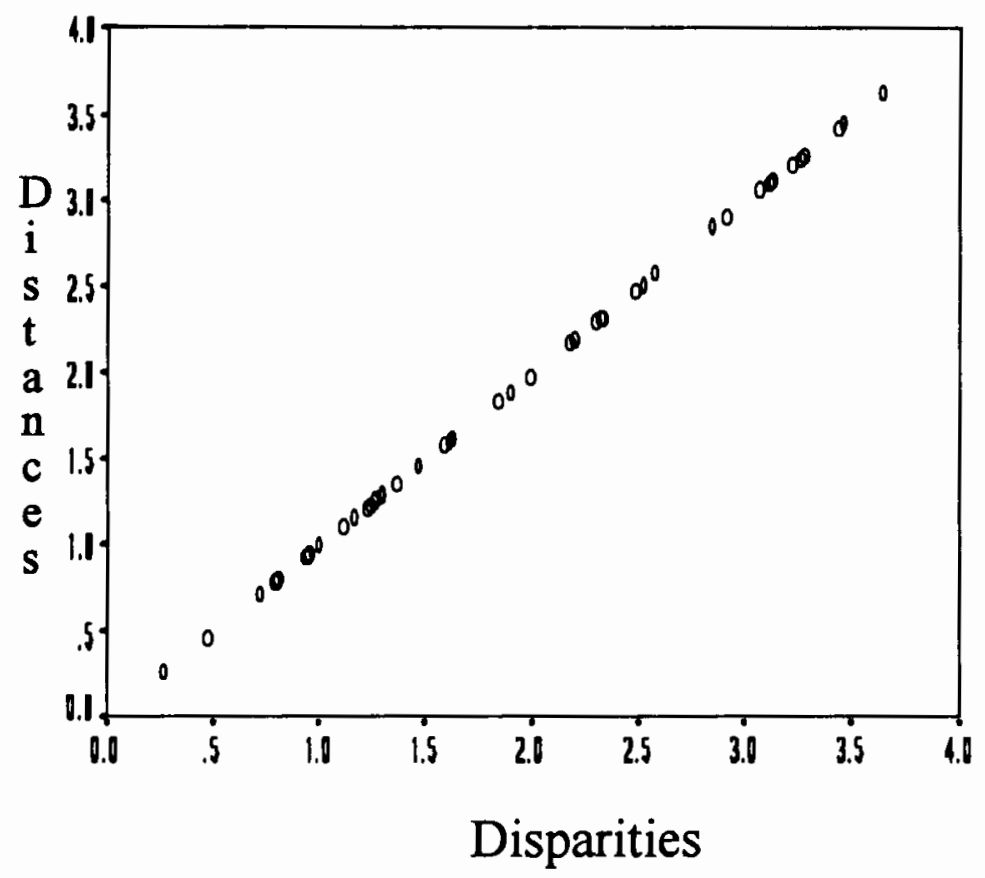

Figure 18. Scatter plot of disparities for ordinal-level two-dimensional map 
When one compares the stress measures for the interval and ordinal maps and (.00050 and .00233 respectively) along with their respective scatter plots, it becomes clear how closely the results of these two procedures correspond. As a consequence, ordinal data, such as that involved in subjective clustering (e.g., card sorting) can be used to obtain interval-scaled configurations which correspond closely enough to those derived from metric data to be interpreted as functionally equivalent. Though one should note that accepting the output of "any monotone relation" also means that some solutions will correspond to the matrix better than others--for this reason, a non-metric approach relies heavily on the analysis of stress measures to assess the quality of the mapping process.

In short, a non-metric scaling approach allowed the ordinal data from a subjective clustering procedure to be used as input for the mapping procedure, and still have the dimensions of the MDS output configuration appear on an interval scale. However, this conclusion also necessitates one cautionary note. Even though the actual distances in a map are on an interval scale, they should not be interpreted to be metrically meaningful. Since the actual input was ordinal, only statements about the relative orderings of items are justified.

The co-occurrence matrices were analyzed using an ordinal-level ALSCAL MDS model. An ALSCAL procedure transforms the similarity data into distances and then plots the labels into a Euclidean space as a set of ordered coordinates. The cooccurrence matrices for each map were also analyzed using a hierarchical clustering 
procedure. The resulting coordinates from the MDS mapping formed the input for the hierarchical clustering procedure and the clustering was performed using a Ward's Euclidean distance model. The results of the clustering procedure were superimposed onto the MDS maps and the combined representations were interpreted.

\subsubsection{Interpretation of the Results}

The results were interpreted on three general levels. First, I examined how the labels were grouped by the hierarchical clustering procedure. My main concern was how those labels associated with the different ethnic identities and different language identities were clustered. For example, were those labels associated with specific ethnic identities (e.g., Vietnamese, Chinese, Asian American) and those associated with specific languages (e.g., speak Vietnamese, speak Chinese, speak English) clustered exclusively in separate groups or were they clustered in the overlapping group(s)? Of particular interest was the nature of the cluster containing MYSELF. "On the assumption that perceived similarity [between a label and MYSELF] would be an indication of identity" (Taylor, et. al., 1973, p. 186) the relative positioning of labels in relation to MYSELF were assumed to reveal the relative strength, or self-salience, of each of the identity labels.

Secondly, by inspecting the distribution of individual labels and clusters of labels within the MDS space, I attempted to determine the attributes of the perceptual space. I had expected that the space would probably be divided in some way along the attributes of language (Vietnamese versus English--though this may include other 
languages as well) and ethnicity (Vietnamese versus a number of other possible ethnicities: i.e., American, Vietnamese-American, Chinese, Asian, etc.). However, this was only speculation and other attributes might have revealed themselves as empirically more important.

Third, by examining the relative position of MYSELF within the MDS space, I examined the relative self-salience of the dimensional attributes (Taylor et. al., 1973). Last, the ranked frequencies for each domain were used to determine which clusters and dimensions were most important for specific domains.

\subsection{Summary of Methodology}

An over-inclusive list of identity labels was generated using multiple administrations of a modified TST. The labels were placed on cards--one each card. In addition, one card was labeled MYSELF and was inserted into the pack. The cards were sorted by the subjects into piles on the basis of perceived similarity. Subjects were allowed to use as many piles as they wished, including a MISCELLANEOUS category, if it was necessary. Subjects also had the opportunity to assign a single label to more than one group by using more than one copy of each label. The groups were labeled by the subjects and five cards were chosen for each of five domains.

The resulting sorts were transformed into an aggregated matrix of cooccurrences and a non-metric ALSCAL MDS analysis was performed on the matrix. A hierarchical cluster analysis was also performed on the data and the results were superimposed onto the MDS configuration. Finally ranked frequencies of labels which 
were chosen for each domain were used to describe the domain specific salience of the clusters and dimensions resulting from the clustering and scaling analyses.

\subsection{Why MDS was Suitable for the Study of Ethnic and Language Identities}

Technically, the question of appropriateness is one of construct validity. That is, did the measurement instrument measure the construct it was proposed to measure in a way that was consistent with the theoretical framework which was being used to pose the research question (Jaeger, 1990, p. 80)?

There are two basic issues in the validity of using MDS for the present study. First, there is the issue of the validity of MDS models of psychological distance. The details of the literature in this field are far beyond the scope of this discussion.

However, there is extensive empirical evidence that the most basic processes of recognition, identification and classification of perceptual stimuli are consistent with the basic axioms of a distance model and that an MDS model is extremely robust (Davison, 1982; Nosofsky, 1992).

The second issue is whether an MDS model is consistent with the theoretical model of identity which was used to pose the research question. Since schema theory was the theoretical framework within which MDS was developed and I used a schema theory of identity, an MDS approach was thoroughly consistent with the theoretical framework of the study. The present framework incorporated both an informationprocessing approach to the self and a social-identity theory of ethnicity. In both of these approaches there is basic agreement that schema development and processing are 
fundamental to self-identity and self-other categorization and comparison. Within an information-processing approach, the self is conceptualized as a system of selfschemata and the self is modeled as a central node, or space, within a network/space of self-salient information. Similarly my adaptation of social identity theory conceptualized ethnicity as one level of differentiated social schemata and also used a spatial model of the relationships involved.

As a psychological methodology, the use of MDS assumed that the objects of comparison are schemata and represents these relationships specifically using a spatial model. Therefore, generally, MDS was the most theoretically consistent instrument for the measurement of the proposed identity comparisons.

Further, the specific MDS methods that were chosen either avoided or solved several methodological problems in the measurement and assessment of these constructs. First, ethnicity is highly variable in both content and its relationships to other identities. Second, the perceived characteristics which belong to various groups often overlap between groups. Lastly, the expression of ethnicity healthfully varies from context to context and it is highly sensitive to the social audience of its expression. All these aspects of ethnicity make it highly undesirable to use many instruments for measuring ethnicity. As Wallman (1983) has observed, the use of behavioral or belief scales tend to be a highly unreliable indicators of identity because:

Individuals do not necessarily identify themselves by the way they look, or the way they behave, or the people with whom they associate; nor do they necessarily identify with everyone or anyone they resemble in phenotype or 
culture. . . [ [Further,] . . the significance put upon external signs of similarity by nonmembers "outside" may well differ from their meaning for members "inside."

$$
\text { (Wallman, 1983, p. 77) }
$$

Therefore, one should generally avoid the use of any instruments which directly infer identity based on the presence of external aspects of culture. This restriction causes some difficulty for research on ethnicity. Researchers' access to these constructs is limited only to what people do and say. if behavior is often an unreliable indicator of identity, then what other alternatives are there? Collecting self-labeling data avoids some of the more obvious problems of inferring identities based on external culture. However, self-labeling or self-reports offer their own set of methodological problems.

On the one hand, open-ended solicitations to the question of self-identification are not contextualized and so tend to produce many irrelevant answers. This leaves the researcher with the responsibility of reducing the data to that which is most relevant. However, which characteristics are most relevant to a person's identity is often the very question that is being asked.

On the other hand, directly questioning people about their ethnicity using a predetermined range of identity labels again places the most important step--deciding the range of possible identities and the selection of labels for those identities--in the hands of the researcher. Researchers may not correctly identify the most salient choices, or characterize them appropriately. Compounding the problem, people tend to acquiesce to their perceptions of what the researchers want. Therefore, "what is needed . . . is a procedure which avoids asking the very vague question "What are 
you?" while at the same time does not directly question people about their ethnic affiliation" (Taylor, 1975, p. 168).

The present research avoided the above problems by using multiple administrations of different versions of the TST which focused on different levels of identity. The TSTs provided the different levels of directness, while taking the intersection of the TST results provided the needed data reduction in a manner which reduced the level of researcher influence in the process. In this way, the range of identity choices, the characterizations of those choices, and the decisions of relevancy were determined by the subject population itself. Allowing these decisions to emerge from the judgments of the subjects was most consistent with the phenomenological principles of the identity theories which were used in the framework of the study.

Consistency with phenomenological principles was also maintained by using an attribute-free approach in the design of the card-sort task and the subjective approach in the interpretation of the resulting maps. Because the dimensions of identity which are most salient are highly variable from individual to individual and group to group, it was best to use an approach which did not predetermine the dimensions of judgment for the subjects, or predetermine the dimensions of interpretation of the results. Since MDS is very well suited "as a means of obtaining comparative evaluations of objects when the specific bases for comparison are unknown or undefinable" (Dillon \& Goldstein, 1984, p. 321), its choice provided the desired flexibility. In these cases the strength of perceptual mapping is its "ability to infer dimensions without the need of 
defined attributes" (Hare, et. al., 1992, p. 321). Therefore, the nature of the subjects' task and the interpretation procedure used to assess the results were also consistent with the theoretical framework of the study. 


\subsection{Presentation and Discussion of Results}

Since the study had three distinct types of data collection and analysis, I will divide the next section into three parts. The first part will present the results of the TST; the second part will present the analysis of the card-sort data, and the third will present the results of the domain specific importance of labels.

\subsection{Selection of Relevant Self-descriptions}

The first research question in the study was as follows:

RQ1: What items are relevant to the subjects' ethnic identities? Are any languages relevant to the subjects' ethnic identities? If so, which languages are relevant?

Therefore, the goal of the first stage of the study was to produce an over-inclusive set of descriptions related to the subjects' ethnic and linguistic identities. Thirty-eight individuals completed the three Twenty Statements Tests (TST). The subject population was purposely varied. There were 17 males and 25 females in the sample. Their ages ranged from 18 to 30 with the mean age being 22 . The amount of time in the US ranged from 4 to 20 years with the mean time in country being 10 years.

This TST stage produced approximately 1,250 self-descriptions. Some were written in English, some in Vietnamese, and some in both languages. Initially, the aggregated results of each TST were placed into separate coded data tables and then a semantic grouping procedure was performed. The process consisted of an iterative process of semantic grouping (described in section 4.4.1, pp. 95-99). The purpose of the procedure was to sort the data into categories of similar meaning. The final result 
of this process was a table of grouped descriptions which contained 18 main categories. A summary table of the categories along with the item frequencies on each TST was used to produce 45 labels which represented the range of items in the categories. This table is presented below as Table VII.

\begin{tabular}{|c|c|c|c|}
\hline Category & TST1 & TST2 & TST3 \\
\hline 1. Vietnamese & 21 & 9 & N/A \\
\hline 2. Born in Vietnam / From Vietnam & 10 & 6 & N/A \\
\hline 3. Chinese & 4 & 3 & N/A \\
\hline 4. Asian & 3 & 4 & N/A \\
\hline 5. Americanized & 1 & 2 & N/A \\
\hline $\begin{array}{l}\text { 6. Comments on time in US } \\
\text { 6.1 Been in the US only a few years } \\
6.2 \text { Been in the US several years } \\
\text { 6.3 Been in the US for many years }\end{array}$ & $\mid \begin{array}{l}3 \\
3 \\
4\end{array}$ & $\begin{array}{l}1 \\
1 \\
1\end{array}$ & N/A \\
\hline $\begin{array}{l}\text { 7. Physical appearance } \\
\text { 7.1 Short } \\
\text { 7.2 Black hair } \\
\text { 7.3 Brown / black eyes } \\
7.4 \text { Tan /dark skin } \\
\end{array}$ & $\begin{array}{l}6 \\
7 \\
5 \\
1 \\
\end{array}$ & $\begin{array}{l}4 \\
1 \\
1 \\
2\end{array}$ & N/A \\
\hline $\begin{array}{l}\text { 8. Language comments } \\
\text { 8.1 Speak English } \\
\text { 8.2 Speak Vietnamese } \\
\text { 8.3 Speak Chinese } \\
\text { 8.4 Speak Vietnamese and English } \\
\text { 8.5 Speak Chinese and Vietnamese } \\
\text { 8.6 Speak English and Chinese } \\
\text { 8.7 Speak Vietnamese, Chinese, English } \\
\text { 8.8 Speak Vietnamese, English, French } \\
\text { 8.9 Don't know English very well } \\
\text { 8.10 Learning Chinese }\end{array}$ & $\begin{array}{l}1 \\
2 \\
1 \\
2 \\
1 \\
1 \\
2 \\
2 \\
3 \\
2\end{array}$ & $\begin{array}{l}1 \\
4 \\
1 \\
3 \\
0 \\
0 \\
2\end{array}$ & $\begin{array}{l}38 \\
38 \\
28 \\
33 \\
25 \\
27 \\
24 \\
9 \\
1 \\
1\end{array}$ \\
\hline 9. Proud to be Vietnamese & 7 & 3 & N/A \\
\hline $\begin{array}{l}\text { 10. Group Attributes } \\
\text { 10.0 Kind } \\
\text { 10.1 Honest } \\
\text { 10.2 Generous } \\
\text { 10.3 Polite } \\
\text { 10.4 Hardworking / perservering }\end{array}$ & $\begin{array}{l}1 \\
2 \\
3 \\
2 \\
5\end{array}$ & $\begin{array}{l}3 \\
4 \\
3 \\
4 \\
5\end{array}$ & N/A \\
\hline
\end{tabular}




\begin{tabular}{|l|l|l|l|}
\hline \multicolumn{1}{|l|}{$\begin{array}{l}\text { 10.5 Religious } \\
\text { 10.6 Quiet }\end{array}$} & 5 & 1 & \\
\hline $\begin{array}{l}\text { 11. Comments on Food } \\
\text { 11.1 Likes Vietnamese food }\end{array}$ & 7 & 8 & \\
$\begin{array}{l}\text { 11.2 Likes Chinese food } \\
\text { Nikes American food }\end{array}$ & & & \\
\hline 12. It is important to respect parents / elders & 2 & 7 & N/A \\
\hline 13. Have traditional values & 2 & 9 & N/A \\
\hline 14. Comments on the importance of education & 9 & 4 & N/A \\
\hline $\begin{array}{l}\text { 15 Culture comments } \\
\text { 15.1 Culture is Vietnamese }\end{array}$ & 4 & 2 & \\
15.2 Culture is Chinese & 1 & 3 & \\
15.3 Culture is a mix & 2 & 3 & N/A \\
$\begin{array}{l}\text { 15.4 Comparative / evaluative statements } \\
\text { 15.6 Comments on strategies for cultural adaption }\end{array}$ & 1 & 5 & \\
15.7 Culture is Americanized & 1 & 3 & \\
\hline 16 Want to preserve and teach traditional culture \\
and values & 5 & 2 & \\
\hline $\begin{array}{l}\text { 17.0 Comments on social contacts / network } \\
\text { 17.1 Have lots of Vietnamese friends }\end{array}$ & 1 & 3 & N/A \\
17.2 Have lots of non-Vietnamese friends & 1 & 3 & \\
17.3 Prefer to Marry same race and culture & & 5 & \\
\hline $\begin{array}{l}\text { 18.0 Comments on Being in the US } \\
\text { 18.1 Prefer to live in the US } \\
\text { 18.2 Prefer to live in Vietnam } \\
\text { 18.3 I want to Visit Vietnam }\end{array}$ & 3 & 1 & N/A \\
\hline
\end{tabular}

Table VII: Categorized TST results

Production of the labels was guided by two basic principles. First, I followed the principle of over-inclusiveness. Whenever there was a question about whether an item should have been included or whether a category should have been divided into two labels, the more inclusive choice was made. Second, whenever possible, I used words and key phrases for the label that came directly from the original data. In general, most of the labels were exact or close matches to items actually in the data. 
However, there were several labels from the thematic categories which more loosely summarized the varied themes which were present in those categories.

The label "Myself' was added to the list and the summary labels were then translated and back-translated to produce list of paired (English and Vietnamese) labels in Table VIII. Because these labels were eventually divided into two stages for the card-sorting task, they are presented here within two groups.

The list consisted of those descriptions which subjects thought were relevant to their ethnic and linguistic identities. They included labels related to:

1. Group identity

2. Ethnic pride

3. Time in country

4. Culture

5. Language ability

6. Values

7. Preferred country of residence

8. Physical appearance

9. Food preferences

10. Friendship patterns

11. Personal/group attributes

A number of language descriptions were identified as relevant. These included the ability to speak Vietnamese, English, Chinese, and French; they also included references to a limited ability to speak English, Vietnamese, and Chinese. Since multiple copies of each label were used in the sorting task, references to multilingualism (e.g., I speak English and Vietnamese) did not appear as separate labels. Each pair of 


\begin{tabular}{|c|c|c|}
\hline \multicolumn{3}{|c|}{ First group } \\
\hline ID & English & Vietnamese \\
\hline 1 & Myself & Ban thân tôi \\
\hline 2 & Vietnamese & Ngươi Việt Nam \\
\hline 3 & Bom in Vietnam / From Vietnam & Sanh ̛̊ Việt Nam / Tữ Việt Nam \\
\hline 4 & Chinese & Người Trung-Hoa \\
\hline 5 & Asian & Người á đông \\
\hline 6 & Americanized & Người Mỹ hóa \\
\hline 7 & Asian-American & Người Mỹ gốc á đông \\
\hline 8 & Been in the US only a few years & ờ nứcc Mỹ vai năm (thôi) \\
\hline 9 & Been in the US for many years & d̛ nứoc Mỹ nhiêu năm \\
\hline 10 & Speaks English & Nói tiềng $\mathrm{My}$ \\
\hline 11 & Speaks Vietnamese & Nói tiểng Việt \\
\hline 12 & Speaks Chinese & Nói tiếng Trung-Hoa \\
\hline 13 & Speaks French & Nói tiềng Pháp \\
\hline 14 & Speaks English, but not very well & $\begin{array}{l}\text { Nói tiềng Mỹ, nhưng không nói } \\
\text { tiêêng Mỹ ranh }\end{array}$ \\
\hline 15 & Speaks Vietnamese, but not very well & $\begin{array}{l}\text { Nói tiêng Viẹt, nhưng không nói } \\
\text { tiếng Vięt ranh }\end{array}$ \\
\hline 16 & Speaks Chinese, but not very well & $\begin{array}{l}\text { Nói tiêng Trung-Hoa, nhưng không noi } \\
\text { tiếng Trung-Hoa rành }\end{array}$ \\
\hline 17 & Proud to be Vietnamese & Hãnh diện là ngươi Viêt Nam \\
\hline 18 & Culture is Vietnamese & Phong tuc Viêt Nam \\
\hline 19 & Culture is Chinese & Phong tuc Trung-Hoa \\
\hline 20 & Culture is Americanized & Phong tuc Mỹ hóa \\
\hline 21 & Has mixed culture & Có phong tyc lẫn-lộn \\
\hline 22 & Family comes first & Gia đinh trên hết \\
\hline 23 & Has traditional values & Có phong tục truyên thông \\
\hline 24 & It is important to respect parents/elders & Diều cần nhất là phải kính trong cha me \\
\hline
\end{tabular}




\begin{tabular}{|c|c|c|}
\hline \multicolumn{3}{|c|}{ Second group } \\
\hline ID & English & Vietnamese \\
\hline 25 & $\begin{array}{l}\text { Wants to preserve and teach traditional } \\
\text { culture and values }\end{array}$ & $\begin{array}{l}\text { Muôn bảo tồn và dạy bảo phong tục } \\
\text { truyền thống }\end{array}$ \\
\hline 26 & It is important to succeed in education & $\begin{array}{l}\text { Thanh cong trong sự học hânh là một } \\
\text { điều quan trong }\end{array}$ \\
\hline 27 & Prefers to live in the US & Thich sông trong nước $\mathrm{My}$ \\
\hline 28 & Prefers to live in Vietnam & Thich sông ở Viêt Nam \\
\hline 29 & $\begin{array}{l}\text { Wants to visit Vietnam, but not stay } \\
\text { to live }\end{array}$ & $\begin{array}{l}\text { Muôn thăm nước Việt Nam, nhưng } \\
\text { không muôn ở trú tại Việt Nam }\end{array}$ \\
\hline 30 & Short & Tấp (lùn) \\
\hline 31 & Black hair & Toc den \\
\hline 32 & Brown / black eyes & Mằt đen / nâu \\
\hline 33 & Tan/dark skin & Da vang \\
\hline 34 & Has lots of Vietnamese friends & Có nhiều bagn Việt Nam \\
\hline 35 & Has lots of non-Vietnamese friends & $\begin{array}{l}\text { Có nhiều bạn khong phải là ngươi } \\
\text { Việt Nam }\end{array}$ \\
\hline 36 & Likes Vietnamese food & Thich thüch ăn Viêtt Nam \\
\hline 37 & Likes Chinese food & Thich thüch ăn Trung-Hoa \\
\hline 38 & Likes American food & Thích thüch ăn My \\
\hline 39 & Kind & Lōng hảo tâm \\
\hline 40 & Honest & Thât tha \\
\hline 41 & Generous & Rộng lương \\
\hline 42 & Polite & Lich sư (phong nhâ) \\
\hline 43 & Hardworking / perservering & Chiu kho \\
\hline 44 & Quiet & Im lăng \\
\hline 45 & Religious & Đao dúrc \\
\hline
\end{tabular}

Table VIII: Final list of Labels 
labels was printed on a separate " 3 X 5" card. English appeared on one side and Vietnamese on the other. These cards were used for the sorting stage of the study. Several results from the TST deserve special mention. First, in the 1250 selfdescriptions from these 42 subjects, the label "American" never appeared. Instead, subjects always used adjective forms (e.g., Americanized, Americanized Vietnamese, etc.).). Though this may be partially explained by the fact that most of the subjects were relatively recent immigrants, this finding is also consistent with the results from similar groups with a longer history in the US. Yao (1983), for example, studied 94 ChineseAmerican teenagers many of which were second or third generation in the US. She reported that not one subject labeled themselves as American. They chose instead to label themselves as either Chinese or Chinese-American. This was also consistent with Woon's (1985) description of the Vietnamese community in Victoria, Canada. Though most of his sample had been heavily exposed to Western culture in Vietnam, planned to make a permanent home in Canada, and even took on Anglicized names, none of them identified themselves as Canadian (Woon, 1985, p. 543).

Second, the TST results also included many references to Chinese identity and culture. To some extent this may have been a result of the strong Chinese/Confucian influence in Vietnamese culture generally. However, a historical linkage between the two cultures may not completely explain the data. For example, many Americans may recognize a strong historical linkage to England, though being British probably would not commonly appear on American TST results. Woon (1985) has also commented on 
the strong sense of Chinese influence among the Vietnamese immigrants in Victoria, but as he explained, this was mostly due to the demographic diversity within the group normally designated as Vietnamese immigrants. Several groups which were distinct minorities within Vietnam have often been grouped together as Vietnamese refugees or immigrants. However, Woon also argued that very few scholars have even commented briefly "on the intra-group differences among those who are officially defined as 'Vietnamese" (Woon, 1985, p. 538). Among the population of immigrants from Vietnam are a large number of ethnic Chinese and mixed Chinese/Vietnamese families. Though I didn't specifically ask about the ethnic make-up of the subjects' families, the data for the TST results suggested that many subjects may have been from either ethnic Chinese or Chinese/Vietnamese families.

The relationship between the identification and labeling issue came out in the TST data in several ways. I will offer the following quotes as just two examples of the complexity involved. As one subject wrote: "I was born in Vietnam, but my parents say that I'm actually Chinese" (Subject 18 ). Another wrote: "I think that I am very Vietnamese because I was born there and speak the language, but my parents want me to identify myself as Chinese because they say that my Grandparents just immigrated from China to Vietnam. I prefer to be Chinese" (subject 19). During an informal conversation with this last individual, she described how her parents had moved from Vietnam to the US because they believed that their family could maintain its Chineseness better in the US. This situation was also mentioned in Woon's (1985) study and 
may express an attitude that was relatively common within many Chinese/Vietnamese families. It seems that many Chinese within Vietnam left the country both to find greater opportunity and also to avoid the discrimination of post-war Vietnamese nationalism (Woon, 1985). They often thought that the political environment of the US would give them greater freedom to raise their families as they wished.

\subsection{Results of card-sorting procedure}

\subsubsection{General description}

The second stage of data collection and analysis was designed to answer the following research questions:

RQ 2: How did subjects categorize those things which they described (in the TST results) as important to their ethnic and linguistic identities? What were the basic identity categories for the subjects? Did they use primarily ethnic, racial, national, or language categories; or did they use some other manner of organization? What attributes, behaviors, languages, values, etc., were included in the different categories?

RQ3: What were the criteria that subjects used to structure their ethnic and linguistic identity choices?

RQ4: How were the languages indicated by the subjects (if any) related to the various ethnic identities involved? Did subjects uniquely associate particular languages with particular ethnic identities, or did the languages 
show mixed associations with a number of ethnic identities? In particular, how were the subjects' English-speaking identities related to their various ethnic identity options? How did English compare with the other language options available?

There were 30 subjects for the card-sorting stage of the study. Some of these subjects also participated in the first stage of the study. Of these, 16 were male and 14 were female. The subjects were all born in Vietnam, but were living in the US. Their ages ranged from 20 to 30 years old with the median age category being 24 years old. All of the subjects were enrolled in university level classes with the majority having at least junior standing. The length of residency for the subjects ranged from 4 to 20 years with the mean length of residency being 11 years.

The subjects individually sorted the descriptions obtained from the TST stage of the study into groups on the basis of perceived similarity. The labels were divided into two different data sets. Each set was entered into SPSS for Windows and transformed into aggregated similarity matrices using an SPSS matrix program specially written for the purpose. ${ }^{39}$ Each similarity matrix was analyzed separately using the multidimensional scaling and hierarchical clustering procedures available in SPSS.

The first data set consisted of the co-occurrence data for the full 45 labels. Since the sorting task involved placing the labels into groups of similar meaning that represented kinds of people, the first mapped space was understood as representing 
that portion of the social world which was perceived by the subjects as relevant to their ethnic/cultural identity.

The second data set was produced by including only those groups from the original card-sort data which contained the label "Myself." These data were mapped and clustered separately. Whereas the social map represented the self as a uniquely placed point within a perceived social world, this map represented the self as a space within which various cultural influences were more or less self-salient.

The results of each mapping procedure will be discussed in turn. Each data set was mapped in 1 to 5 dimensions. Stress measures were used to identify preferred levels of spatial dimensionality. In each case, the resulting co-ordinates were used to perform a hierarchical clustering procedure on the labels. Finally, the results of the hierarchical clustering were superimposed onto the respective MDS spaces.

\subsubsection{Mapping the Subjects' Perceived Social Space}

The results of the 45 label sorting were mapped in 1 to 5 dimensions. The stress statistics for each dimension are presented in Table IX and a scatter plot of the information is presented in Figure 19. Based on the stress statistics, both two and three-dimensional solutions were mapped and examined. Since the three-dimensional maps retained the basic structure of the two-dimensional solution and it also added an interpretable third dimension, the three-dimensional solution was chosen for final interpretation. The co-ordinates of the three-dimensional solution were entered into SPSS and a hierarchical cluster analysis was performed using a Ward's Euclidean 


\begin{tabular}{|c|c|c|}
\hline Dimensions & Stress & RSQ \\
\hline 1 & .3972 & .5532 \\
\hline 2 & .2169 & .8195 \\
\hline 3 & .1642 & .8775 \\
\hline 4 & .1319 & .9094 \\
\hline 5 & .1083 & .9300 \\
\hline
\end{tabular}

Table IX: Stress and RSQ statistics for social map-1 through 5 dimensions

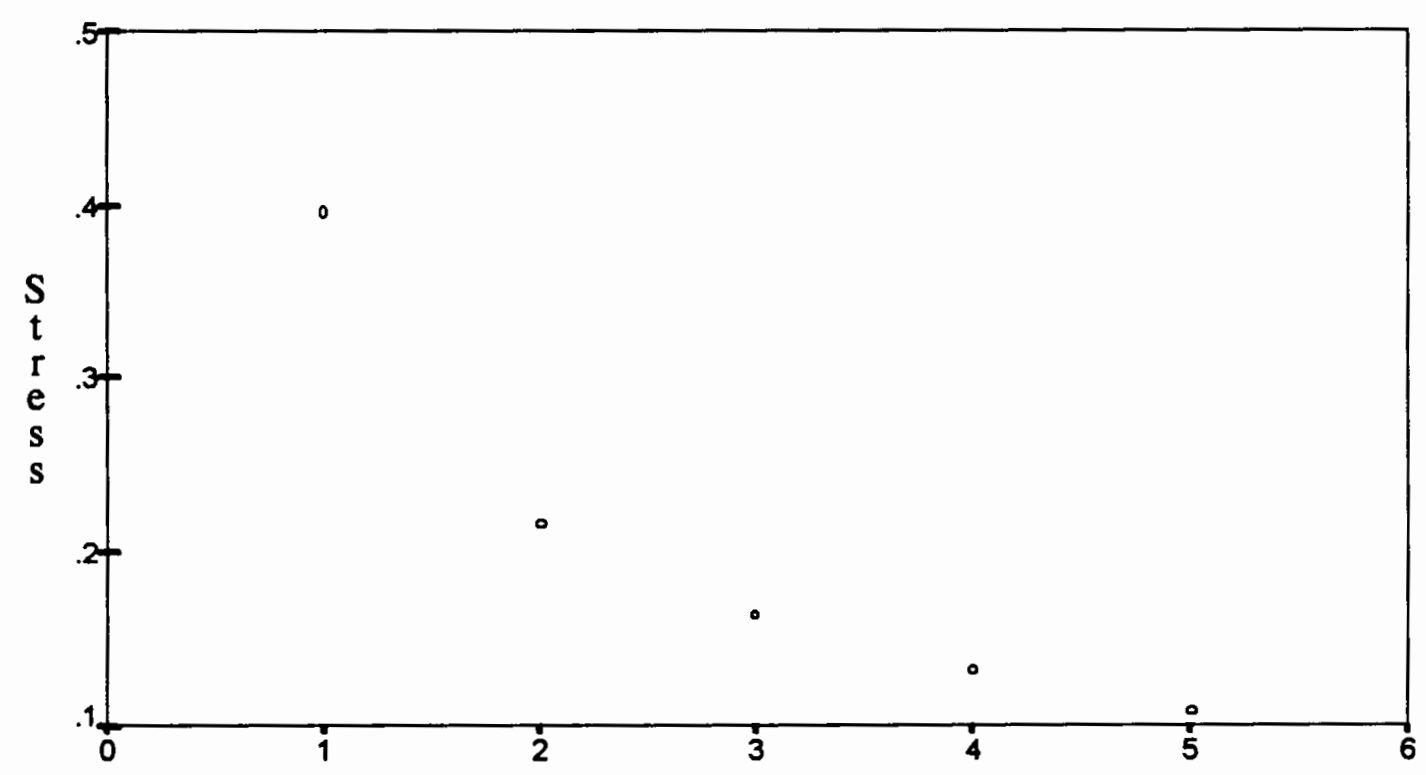

Number of dimensions

Figure 19: Social Map--scatter plot of stress and number of dimensions

distance model. The resulting agglomeration schedule and dendogram are presented in Table $\mathrm{X}$ and Figure 20 respectively. Visual inspection of the agglomeration schedule and the dendogram revealed a meaningful jump in the distance coefficient between stages 40 and 41 of the agglomeration process-highlighted in Table X. Therefore, the process was stopped after stage 40 and a five- cluster solution was chosen. This solution is represented on the dendogram by a broken vertical line. 
Clusters Combined

Stage Cluster 1st Appears

\begin{tabular}{|c|c|c|c|c|c|c|}
\hline \multicolumn{4}{|c|}{ Clusters Combined } & \multicolumn{2}{|c|}{ Stage Cluster 1st Appears } & Next \\
\hline Stage & Cluster 1 & Cluster 2 & Coefficient & Cluster 1 & Cluster 2 & Stage \\
\hline 1 & 42 & 43 & .021309 & 0 & 0 & 4 \\
\hline 2 & 26 & 32 & .044413 & 0 & 0 & 5 \\
\hline 3 & 31 & 41 & .080144 & 0 & 0 & 5 \\
\hline 4 & 39 & 42 & .127516 & 0 & 1 & 7 \\
\hline 5 & 26 & 31 & .188097 & 2 & 3 & 6 \\
\hline 6 & 26 & 37 & .270171 & 5 & 0 & 16 \\
\hline 7 & 25 & 39 & .366436 & 0 & 4 & 18 \\
\hline 8 & 8 & 14 & .466502 & 0 & 0 & 35 \\
\hline 9 & 7 & 9 & .592020 & 0 & 0 & 28 \\
\hline 10 & 22 & 33 & .745461 & 0 & 0 & 15 \\
\hline 11 & 2 & 11 & .902138 & 0 & 0 & 24 \\
\hline 12 & 10 & 38 & 1.071726 & 0 & 0 & 22 \\
\hline 13 & 24 & 36 & 1.248356 & 0 & 0 & 18 \\
\hline 14 & 6 & 15 & 1.496502 & 0 & 0 & 25 \\
\hline 15 & 22 & 40 & 1.750054 & 10 & 0 & 21 \\
\hline 16 & 26 & 27 & 2.008246 & 6 & 0 & 23 \\
\hline 17 & 30 & 44 & 2.294801 & 0 & 0 & 26 \\
\hline 18 & 24 & 25 & 2.589606 & 13 & 7 & 23 \\
\hline 19 & 4 & 12 & 2.897857 & 0 & 0 & 30 \\
\hline 20 & 3 & 34 & 3.227981 & 0 & 0 & 31 \\
\hline 21 & 22 & 23 & 3.575258 & 15 & 0 & 32 \\
\hline 22 & 10 & 21 & 3.928304 & 12 & 0 & 28 \\
\hline 23 & 24 & 26 & 4.351860 & 18 & 16 & 29 \\
\hline 24 & 2 & 17 & 4.782792 & 11 & 0 & 34 \\
\hline 25 & 6 & 20 & 5.239219 & 14 & 0 & 37 \\
\hline 26 & 5 & 30 & 5.746734 & 0 & 17 & 39 \\
\hline 27 & 16 & 35 & 6.255785 & 0 & 0 & 40 \\
\hline 28 & 7 & 10 & 6.804618 & 9 & 22 & 37 \\
\hline 29 & 24 & 29 & 7.361852 & 23 & 0 & 33 \\
\hline 30 & 4 & 19 & 7.937796 & 19 & 0 & 40 \\
\hline 31 & 3 & 18 & 8.541230 & 20 & 0 & 34 \\
\hline 32 & 22 & 45 & 9.265388 & 21 & 0 & 33 \\
\hline 33 & 22 & 24 & 10.008252 & 32 & 29 & 39 \\
\hline 34 & 2 & 3 & 11.063679 & 24 & 31 & 36 \\
\hline 35 & 8 & 28 & 12.282381 & 8 & 0 & 38 \\
\hline 36 & 1 & 2 & 13.808002 & 0 & 34 & 41 \\
\hline 37 & 6 & 7 & 15.436460 & 25 & 28 & 44 \\
\hline 38 & 8 & 13 & 17.189695 & 35 & 0 & 41 \\
\hline 39 & 5 & 22 & 19.002768 & 26 & 33 & 42 \\
\hline 楼) & 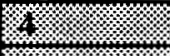 & 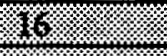 & 46 & 稵 & \% & 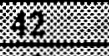 \\
\hline 48 & 格 & 6 & 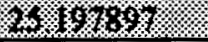 & 好 & 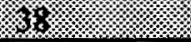 & 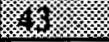 \\
\hline 42 & 4 & 5 & 31.031187 & 40 & 39 & 43 \\
\hline 43 & 1 & 4 & 38.029453 & 41 & 42 & 44 \\
\hline 44 & 1 & 6 & 48.165222 & 43 & 37 & 0 \\
\hline
\end{tabular}

Table X: Agglomeration schedule for clustering of social space labels 


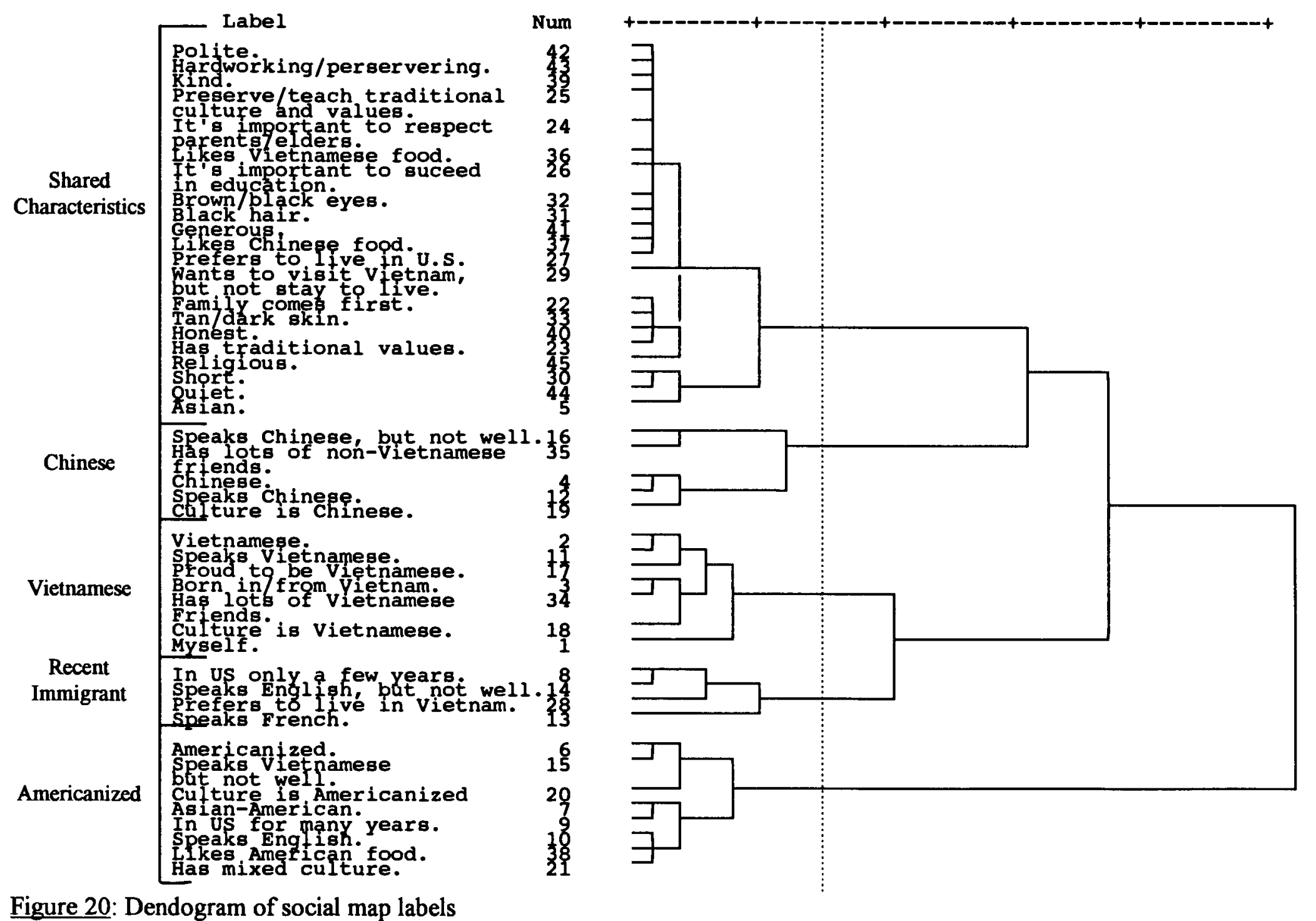


Each cluster was given a summary name by examining (a) the contents of the clusters, (b) the actual names that subjects provided for groupings during their individual card sorts, and (c) the positions of the clusters within the MDS space. The clusters were then superimposed onto the MDS configuration by drawing a boundary around each group of items. The combined configuration is presented in Figures 21 and 22 .

The mapped space can be understood as representing that portion of the social world which was perceived by the subjects as relevant to their ethno-cultural identity. Within this space there were three clusters related to specific ethnic group identities or cultural influences. These were labeled "Vietnamese", "Chinese", and "Americanized." There was also a cluster containing labels related to being a recent immigrant; and a cluster of shared characteristics for the social space as a whole. In other words, those things were perceived as common to all of the different subgroups within the map.

The large cluster of "Shared characteristics" was given its name primarily because of its position within the MDS space. It was mapped into the center of the space because it's contents had an overall high number of co-occurrences with the all of the other labels in the map. This indicated that these items were a shared set of 


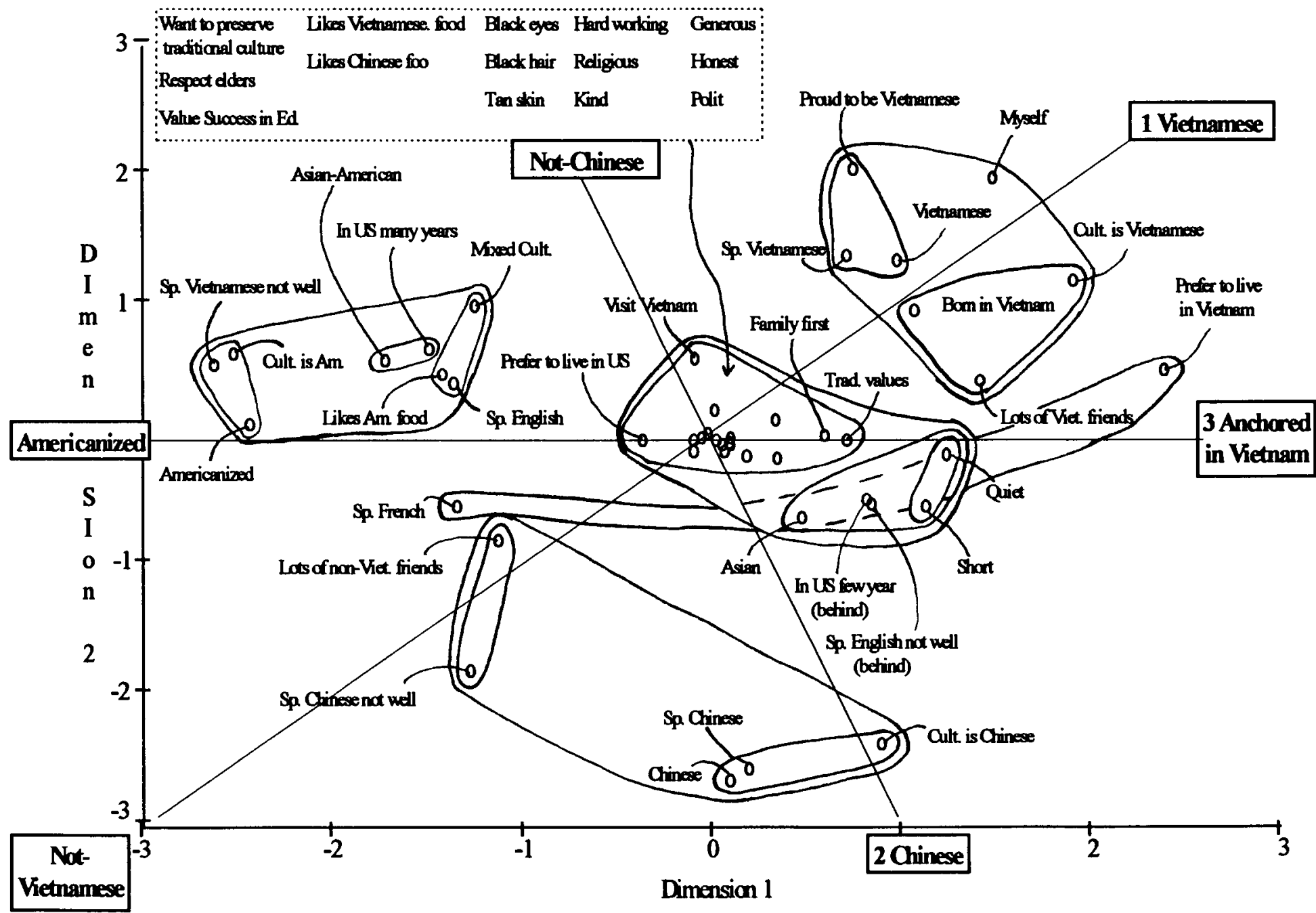

Figure 21: Perceived social world (spatial dimensions 1 and 2) 


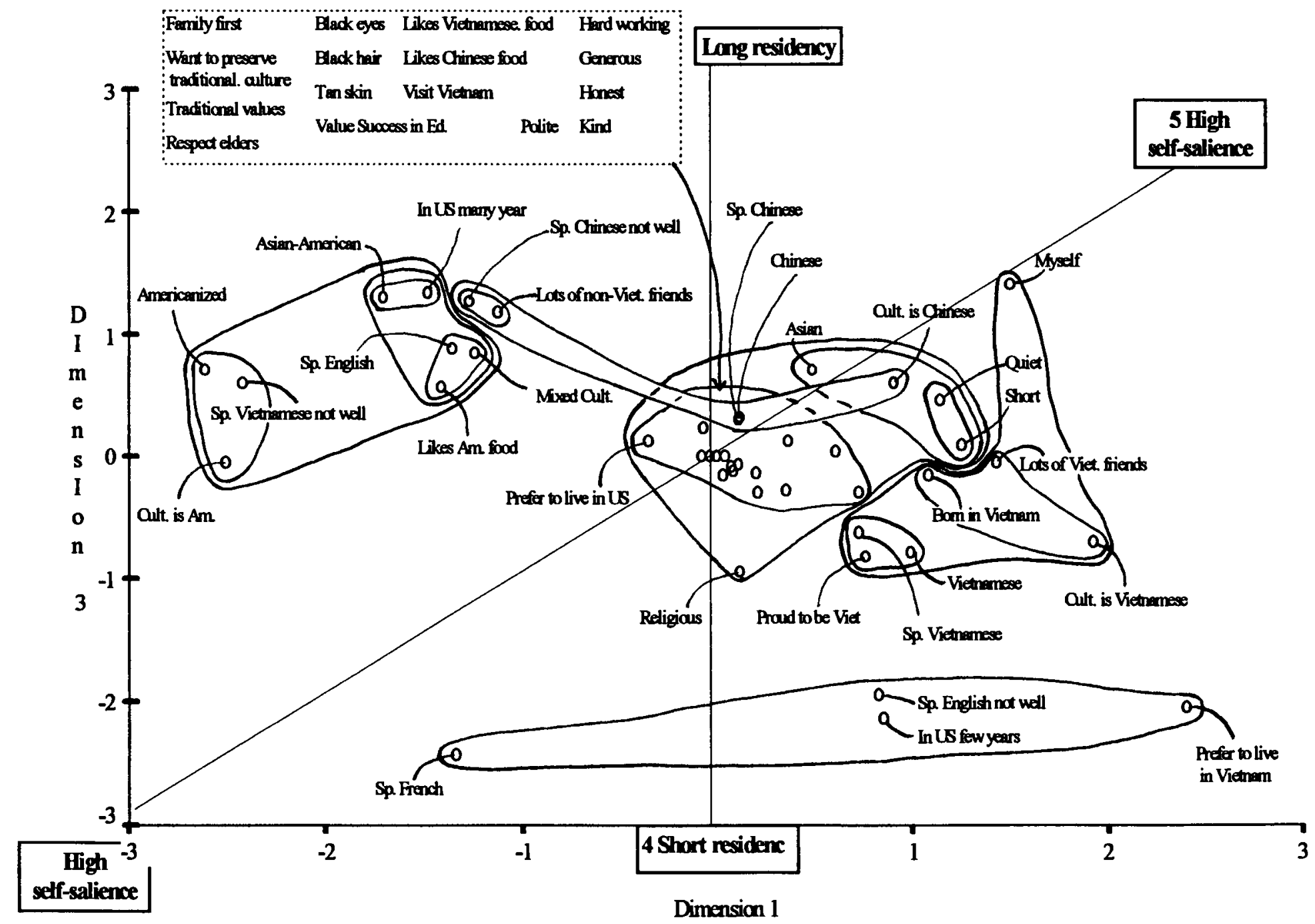

Figure 22: Perceived social world (spatial dimensions 1 and 3) 
concepts for the space as a whole. The cluster was divided into two sub-groups. The most central sub-group contained the following labels:

(Shared residency preferences related to the US and Vietnam)

"Prefers to live in the US"

"Wants to visit Vietnam, but not stay to live"

(Shared values)

"Have traditional values"

"Wants to preserve and teach traditional culture and values"

"Family comes first"

"It is important to respect parents/ elders"

"It is important to succeed in education"

(Shared physical characteristics)

"Black hair"

"Brown/black eyes"

"Tan/dark skin"

(Shared food preferences)

"Likes Vietnamese food"

"Likes Chinese food"

(Shared perceptions of personal/group attributes)

"Kind"

"Honest"

"Polite"

"Generous"

"Hardworking"

An additional sub-group within the Shared Characteristics cluster contained the labels "Asian," "Short," and "Quiet."

Once the clusters were examined in the context of the overall configuration, meaningful dimensions were identified within the space. Often the spatial dimensions in a map roughly correspond to the directions of meaning in a map. However, the number and rotation of meaningful directions in any ALSCAL configuration needn't be restricted 
by the actual spatial configuration of the map. The fact that the number of meaningful dimensions can exceed the number of actual spatial dimensions should not in itself be alarming. It is often common to find that there are several highly compatible interpretations in a space which are distinct yet highly related (Kruskal \& Wish, 1978). In these cases the meaningful dimensions are not orthogonal and hence not represented by additional spatial dimensions. "It should be pointed out that although perpendicular axes are simpler and hence scientifically preferable, nonperpendicular (oblique) axes may often provide a better characterization of the 'real world' (see Harshman, 1970)" (Kruskal \& wish, 1978, p. 43).

Consistent with this possibility, five meaningful dimensions were identified in the space. In order to avoid confusion, I will refer to the actual spatial dimensions of the map as "dimensions" and interpreted directions within the space as "vectors." Vector 1 consisted of a Vietnamese/Not-Vietnamese continuum. Vector 2 consisted of a Chinese/not-Chinese continuum. Vector 3 consisted of an Anchored-inVietnam/Americanized continuum. Vector 4 consisted of a Short/Long-residency continuum; and vector 5 consisted of a self-salience continuum.

The vectors are consistent with previous theory and research on ethnicity in general and Vietnamese identity in particular. Many authors (e.g., De Vos, 1982; Khan et al., 1983; Mahapatra, 1983; Turner, 1985) have emphasized that individuals define their social identity through in-group/out-group distinctions. In general, the structure of the vectors followed this principle by dividing the space into dichotomies of Vietnamese 
vs. Not-Vietnamese, Chinese, vs. not-Chinese, etc. The space also presented a view of multiple identity influences and choices. Baker (1982, as cited in Wallman, 1983) has argued that studies of ethnicity should allow subjects to express multiple identifications. The MDS space is consistent with this argument. If the space had been dichotomized according to a priori categories, much of the richness and real complexity in the situation would have been lost. Further, there would have been no way to know if the most salient information had remained. Allowing subjects to generate the range and structure of options themselves resulted in a more valid (and complicated) reflection of the subjects' ethno-cultural identities.

The particular vectors which were identified in the space were also consistent with previous research on Vietnamese in North America. Several authors have commented on the persistence of Vietnamese ethnic identity (e. g., Celano \& Tyler, 1994; Woon, 1985) and on the community's concern over Americanization (e.g., Celano \& Tyler, 1994; Wolfram et al. 1986). Woon (1985) has also discussed the importance of recognizing a strong Chinese influence within the group's identity. Finally, several authors (e.g., Celano \& Tyler, 1994; Wolfram et al. 1986; Woon 1985) have noted the importance of time in country within the group's perceptions.

While the vectors are generally consistent with past research, a detailed examination revealed a number of complexities in how the subjects viewed their social world. Though vectors 1,2 , and 3 are all related to ethnic group identities, the details of their structures are very different. Discussing each vector in turn will highlight these 
differences. The labels which most strongly determined the Vietnamese end of vector 1 were "Culture is Vietnamese" and Proud to be Vietnamese." Ethnic pride in particular has been identified by several researchers as an indication of positive attitudes towards one's identity (e.g., Keefe \& Padilla, 1987). Consistent with this position, the label "Myself" appeared on the extreme Vietnamese end of vector 1. This positioning of "Myself" along with its membership in the Vietnamese cluster suggested that the subjects possessed a strong and positive sense of Vietnamese group identity. More weakly determining this vector were the labels referring to Vietnamese language, the fact of being born in Vietnam, and access to lots of Vietnamese friends. Labels from both the Chinese and Americanized clusters formed the Not-Vietnamese end of this continuum. Within the Americanized cluster, the labels "Americanized," "Speaks Vietnamese, but not well, " and "Culture is Americanized" most strongly determined the Not-Vietnamese end of the vector. The labels "Mixed culture," "Speaks English," and "likes American food" appeared more neutrally placed on vector 1 . The bulk of the Chinese cluster appeared more neutrally placed on the vector, though within the Chinese cluster, limited proficiency in Chinese and access to lots of non-Vietnamese friends was placed towards the Not-Vietnamese end of the vector.

Vector 2 consisted of a Chinese/not-Chinese opposition. The Chinese end of vector 2 was determined by a relatively tightly anchored set of labels including those referring to group identity, language, and culture. It is notable that even the label "Speaks Chinese, but not well" appeared on the Chinese end of this vector and within the 
Chinese cluster. This would suggest that even a limited ability to speak Chinese was perceived by the subjects as an indication of Chinese identity. The not-Chinese end of this vector contained both the Americanized and Vietnamese clusters. The Vietnamese cluster was more spread out along the vector than the American cluster. Within the Vietnamese cluster there were two distinct sub-groups The sub-group which was farthest to the not-Chinese end of the vector contained the labels "Vietnamese," "Speaks Vietnamese," and "Proud to be Vietnamese." "Proud to be Vietnamese" appeared on the extreme not-Chinese end of this continuum. The sub-cluster containing "Born in/from Vietnam," "Has lots of Vietnamese friends," and "Culture is Vietnamese" was placed more to the Chinese end of vector 2. As a whole, the Americanized cluster appeared more to the not-Chinese end of vector 2 . Within the centrally placed cluster of shared items, the sub-cluster containing "Quiet," "Short," and "Asian" appeared more to the Chinese end of the vector than the other labels in the cluster.

Vector 3 consisted of an Americanized/Anchored-in-Vietnam continuum. Most strongly determining the Americanized end of the continuum were the labels Americanized," "Culture is Americanized", and "Speaks Vietnamese, but not well." "Asian-American" and "In the US many years" more weakly determined the vector. Even more neutrally placed were the labels referring to a preference for American food, having a mixed culture and the ability to speak English. The Anchored-in-Vietnam end of the vector was determined by the labels "Prefers to live in Vietnam," "Culture is Vietnamese," and "Has lots of Vietnamese friends." The Vietnamese and Chinese 
clusters generally appeared toward the Anchored-in-Vietnam end of the vector with the Vietnamese cluster being more strongly so. Within the Chinese cluster, "Lots of Vietnamese friends" and "Speaks Chinese, but not very well" appeared solidly on the Americanized end of the vector. Within the centrally placed cluster of shared items, the sub-cluster containing "Short," "Quiet," and "Asian" appeared more to the Anchored-inVietnam end of vector 3 .

The dichotomy represented in the Americanized/Anchored-in-Vietnam vector was also reported in Woon's (1985) study. Woon reported that a major distinction within the community that he studied was between those who kept up with the politics of Vietnam and wished to return if they could and those who had chosen Victoria as their permanent home both physically and psychologically. In this study, the distinction came up during one data collection session at a Vietnamese student organization meeting. The meeting centered around whether the organization should be involved in a local cultural celebration. Some felt that the celebration would be too focused on their parents' political ties to Vietnam (a tie that was not strongly felt by all of the members) and others felt that concern over Vietnam should be an important aspect of Vietnamese identity. By the end of the meeting the topic had not been decided and it was scheduled for further discussion.

Vector 4 (Figure 22) consisted of a length-of-residence vector. This vector was anchored on the Short-residency end by labels indicating low English proficiency and a short time in the US. Generally, the Vietnamese cluster was placed more on the Short- 
residency end of this vector. Within the Vietnamese cluster, the labels "Vietnamese," "Culture is Vietnamese," and "Proud to be Vietnamese" appeared most to the Shortresidency end. Within the Chinese cluster, most of the labels were placed neutrally along the vector, with the exception of "Speaks Chinese, but not well" and "Has lots of Vietnamese friends." These were placed on the Long-residency end of the vector. The Americanized cluster (and especially the Asian-American sub-cluster) appeared on the Long-residency end of the continuum. Farthest to the Long-residency end of the vector were labels referring to being in the US a long time and being Asian-American. Ordered more neutrally, but still on the Long-residency end of vector 4 were the labels of "Speaks English," "Mixed culture," and "Likes American food." Within the Shared characteristics cluster, most of the labels-including "Prefers to live in the US"--were placed very neutrally along the vector. However, "Asian" was placed more towards the Longresidency end of the vector and "Religious" was placed more toward the short-residency end of the vector than any other label in the cluster. Also on the Long-residency end of the vector was the label "Myself." The fact that "Myself" was within the Vietnamese cluster, but appeared by itself on the Long-residency end of the vector would suggest that the criterion was applied differently on the group and individual levels. Whereas the subjects perceived the Vietnamese cluster as more toward the Short residency end of the vector than either the Chinese or Americanized clusters, the subjects thought of themselves, individually, as having been in the US for many years. 
Again, Woon (1985) also noted that being a recent versus a long-time immigrant was a salient social distinction within the community in Victoria. He reported that long-term immigrants (whether in the more general Chinese community or within the Vietnamese community itself) often distinguished between, and discriminated against, more recent immigrants from Vietnam. Further, he also noted that this awareness extended to the group level. Both Vietnamese immigrants and the larger Chinese community had the perception that the Chinese were the longer-established group and therefore of higher status.

Vector 5 (Figure 22) was interpreted as a self-salience continuum. On the High self-salience end of vector 5 appeared the label "Myself." An examination of the similarity matrix revealed that those items which collectively co-occurred least with "Myself" ("Speak French," "Americanized, Culture is Americanized," and "Speak Vietnamese not well") appeared on the opposite end of the space. However, beyond its presence, vector 5 was difficult to assess because only a few labels appeared meaningfully separated along the vector. It could be that the vector itself was multifaceted in a way which the social map could not fully capture. Another possibility is that this vagueness was partly produced by the mapping procedure itself. Very generally, the mapping procedure placed those items with the highest levels of co-occurrence closest to each other in the space. However, this is only partly true. In actuality, only those labels which most uniquely co-occurred with "Myself" were positioned closest to it. In the case of the label "Myself," this emphasis on uniqueness was enhanced because the 
sorting task allowed all of the labels except "Myself" to have multiple group

memberships. This sometimes meant that a label with a high number of co-occurrences with "Myself" could appear toward the center of the space because it co-occurred just as highly with labels in other parts of the space.

This points out an important constraint on how the map should be interpreted. While the map presented a good representation of the structure of the subjects' perceived social world and it generally indicated which clusters or vectors were most uniquely associated with self, it could not be used to assess the relative self-salience between individual items in the space. That is, the distances between "Myself" and other labels were not valid reflections of the relative self-salience of those items. This was not anticipated in the design of the study and was discovered through the interpretation of the map. Since the issue of self-salience was important to the overall goals of the study, an alternative method was devised to assess the relative self-salience of individual labels. This involved the use of a sub-set of the data and a separate mapping procedure. The details of this process are presented below (p. 143). However, before moving to this topic, several important aspects of the perceived social space still deserve discussion.

On the surface, the fact that the subjects divided much of the space according to the three ethno-cultural influences included in the labels (i.e., Vietnamese, Chinese, Americanized) was not surprising to the researcher. However, the structure of the space indicated that there were important differences in the meanings of the different cultural vectors. That is, which criteria were most important for defining each cultural vector 
were slightly different. For example, the Chinese vector was strongly determined by the labels referring to Chinese group identity, culture, and language collectively. The strength of the relationship between the Chinese-associated concepts was demonstrated by the fact that even the label "Speak Chinese, but not well" was associated with Chinese-ness. In contrast, the Vietnamese vector seemed to be most strongly determined by labels associated with culture and ethnic pride, and less strongly by Vietnamese language. Similarly, the Americanized end of vector 3 was most strongly anchored by labels of group identity and culture and even less strongly by English. In addition, the relationship between the Americanized and Vietnamese ends of Vectors 3 and 1 points out an important asymmetry in the relative importance of language within these different vectors. While neither "Speak English" nor the limited ability to speak English formed the strongest ends of vector 3, the limited ability to speak either Vietnamese or Chinese did strongly determine the Americanized end of the vector. In a parallel fashion, Vietnamese language was not the strongest anchor for the Vietnamese end of vector 1; however, low proficiency in Vietnamese did strongly determine the NotVietnamese end of the vector. It seems that the ability to speak Vietnamese was not most important for defining Vietnamese-ness, but the lack of it was strongly associated with a perception of not being Vietnamese. This may have been because within the group, several subjects came from Vietnamese-Chinese families. Considering that there are Chinese-Vietnamese families in which Vietnamese is spoken, but there are also strong Chinese cultural and linguistic influences, this is understandable. Given this 
situation, the ability to speak Vietnamese would not in itself be sufficient to indicate Vietnamese identity, though the inability to speak Vietnamese may be a strong indicator of a lack of Vietnamese identity. Further, English was not most strongly involved in either the perception of being or not being Americanized, though the inability to speak either Vietnamese or Chinese well was strongly associated with Americanization. More specifically, one can speak English well and still not fall on the extreme Americanized end of the vector, but falling on the extreme American end does imply that one will speak English well and not speak either Vietnamese or Chinese well.

The asymmetries appearent in the space may have had several causes. First, they may have been present simply because what was important for defining each cultural vector was different. However, this does not necessarily explain either the asymmetries that were appearent within a single vector, or systematic differences in the relationships between the vectors. Another possibility is that some asymmetries were due to the complex interplay in the orientations of the vectors. Early assimilation theories related to ethno-cultural identity (e.g., Park, 1950; Schuman, 1976,1978) have emphasized oppositional relationships between cultural influences, while more recent theories of ethnic identity (e.g., Berry, 1986; Felix-Ortiz, Newcomb, \& Myers, 1994; ) have emphasized the orthogonal nature of cultural influences. The presented map suggests that which model is the more appropriate representation of a group's perceptions cannot be decided a priori. Further, the two models are not necessarily exclusive of each other. As a whole, the subjects' perceived social space didn't clearly conform to either an 
oppositional or orthogonal pattern. Instead it was a complex combination of relationships which were different depending on which pair of vectors was considered. In reality, the distinction between these types of relationship is continuous rather than dichotomous and this was also evident in the data. Within the relationships between the three vectors, some are clearly oppositional, some seemed more orthogonally related and some seemed mutually supportive. For example, vectors 1 and 3 were related in such a way that being Americanized and being Vietnamese were conceptualized consistent with an assimilation model in which a gain in one meant a loss in the other. On the other hand, the relationship between vectors 2 and 1 seemed more closely orthogonal. There were interpretable sets of labels in all four quadrants representing (a) Chinese/ Vietnamese, (b) Not-Chinese/Not-Vietnamese, (c) Chinese/Vietnamese, and (d) NotChinese/Not-Vietnamese. Finally, vectors 2 and 3 were more orthogonally related than 1 and 3 , but less orthogonally related than 2 and 1 . That is, being Chinese was more associated with being anchored in Vietnam, than being Americanized, but less so than being Vietnamese.

Another way of understanding the pattern of differences can be provided by the following. Assume that the community of individuals is defined on the basis of the shared characteristics in the center of the space (e.g., born in Vietnam, prefer to live in the US, etc.). In addition, treat the vectors in the map as prototype guides along which sub-groups are distinguished. Given these assumptions, one can understand the map as a description of how likely it is that various individuals will be perceived by the group 
along the each vector. That is, the more items that are included in an individual's description which fall on the extreme end of a vector (e.g., Vietnamese), the more likely that individual would be categorized as being that type of person. If one focus on the relationship between ethno-cultural identity and language, the complex set of relationships for this group can be expressed as follows:

1. A person who speaks English will likely be perceived as a mixture of identities with Americanized as a component (i.e., Americanized-Vietnamese, Americanized-Chinese, Americanized-Chinese-Vietnamese) though it will be uncertain how Americanized the person is.

2. A person who speaks English and does not speak either Vietnamese or Chinese very well will very likely be perceived as very Americanized.

3. A person who speaks Vietnamese, will likely to be perceived as Vietnamese but might also be Vietnamese-Chinese. The person will least likely be perceived as Americanized Vietnamese or Americanized Chinese though the later is more likely than the former.

4. A person who speaks Chinese, will more likely be perceived as Chinese than Vietnamese or Americanized. This will be especially true if he/she speaks Chinese well.

These descriptions are a purposeful simplification of the details which were discussed. It should be strongly emphasized that each cluster and area of the space contains a complex set of items ranging from identity labels to personal group attributes. 
The perception of all of these items collectively contribute to social judgments. Along with issues of language, one also has perceptions of whether people are short, quiet, traditional, like Vietnamese as opposed to American food, etc. All of these perceptions play a role in the making of social judgments and this is true of judgments about one's self as well as others.

The group's perceptions of how its various cultural influences were related to each other were complex and involved important differences in what most strongly defined each cultural influence. Further, there were some differences in what defined the positive and negative aspects of each cultural influence. Lastly, There were also differences in how the influences themselves were related to each other (i.e., whether they were in opposition, independent, or mutually supportive).

The social space also presented a large number of characteristics which were perceived as common to the community. These shared characteristics included a perception of shared physical characteristics, a common set of family values, a common set of food preferences, and a shared set of positive personal/group attributes. Also included in this set of shared characteristics was the identity label "Asian."

At the same time, the social space was separated into unique areas of cultural influence and length of residency. Within this space, the self was placed strongly within the Vietnamese cluster and on the Vietnamese end of the space. However, as pointed out earlier, this map only presented the structure of the perceived social world. It did not address how well the perceived organization of this social space matched the 
structure of their own identities. Nor did it represent which aspects of that social world were most self-salient. In order to address these issues, a self-salience space was mapped and interpreted.

\subsubsection{Mapping the subjects' self-salience space.}

Using the same data set as above, I extracted only those groups from each subject's data which contained the label "Myself." With these data, I produced a new similarity matrix which was mapped and clustered separately. The process directly addressed the fifth research question:

RQ5: How self-salient were the various ethnic and linguistic identities in relation to each other? In particular, how self-salient were the subjects' Englishspeaking identities in relation to the other ethnic and language choices involved?

The raw data from the card sorts were transformed into an aggregated similarity matrix and mapped in 1 to 5 dimensions. The stress statistics for each level of dimensionality are presented in Table XI and a scatter plot of the information is presented in Figure 23.

\begin{tabular}{|c|l|l|}
\hline Dimensions & Stress & RSQ \\
\hline \hline 1 & .421 & .702 \\
\hline 2 & .208 & .881 \\
\hline 3 & .147 & .932 \\
\hline 4 & .121 & .949 \\
\hline 5 & .097 & .966 \\
\hline
\end{tabular}

Table XI: Stress and RSQ statistics for self map-1 through 5 dimensions 


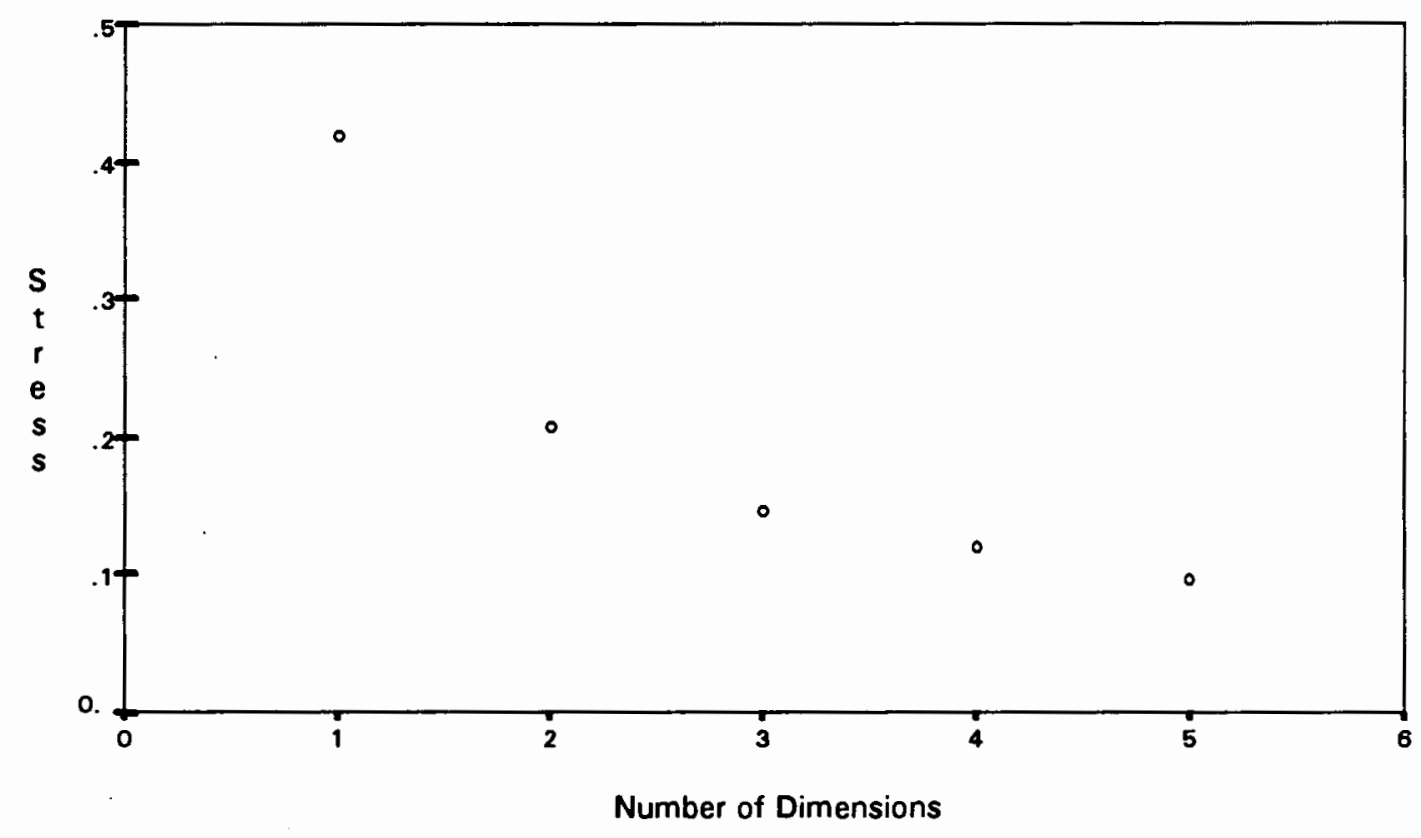

Figure 23: Self-Map--scatter plot of stress and number of dimensions

Based on this information, both two and three-dimensional solutions were mapped for initial interpretation. However, the choice of three dimensions did not add any interpretable information over the two-dimensional solution. The vast majority of labels simply clustered closely in the center of the third dimension and none of the items seemed separated in any way to suggest a useful interpretation. Apparently, the dimension was produced merely to better fit a few weak items. Therefore, a twodimensional solution was chosen for final interpretation. The co-ordinates from the twodimensional map were entered into SPSS and a hierarchical cluster analysis was again performed using a Ward's Euclidean distance model. The resulting agglomeration schedule and dendogram are presented below in Table XII and Figure 24. 


\begin{tabular}{|c|c|c|c|c|c|c|}
\hline & \multicolumn{2}{|c|}{ Clusters combined } & & \multicolumn{2}{|c|}{ Stage cluster lst appears } & Next \\
\hline Stage & Cluster 1 & Cluster 2 & Coefficient & Cluster 1 & Cluster 2 & Stage \\
\hline 1 & 40 & 41 & 000906 & $\mathbf{0}$ & 0 & 4 \\
\hline 2 & 25 & 31 & .002633 & 0 & 0 & 10 \\
\hline 3 & 36 & 37 & .008604 & 0 & 0 & 9 \\
\hline 4 & 32 & 40 & .014866 & $\mathbf{0}$ & 1 & 11 \\
\hline 5 & 22 & 23 & .021978 & 0 & 0 & 8 \\
\hline 6 & 24 & 27 & .031067 & 0 & 0 & 13 \\
\hline 7 & 39 & 42 & .043319 & 0 & 0 & 10 \\
\hline 8 & 22 & 26 & .059828 & 5 & 0 & 11 \\
\hline 9 & 36 & 43 & .077194 & 3 & 0 & 13 \\
\hline 10 & 25 & 39 & .098264 & 2 & 7 & 16 \\
\hline 11 & 22 & 32 & .123378 & 8 & 4 & 12 \\
\hline 12 & 1 & 22 & .152867 & 0 & 11 & 16 \\
\hline 13 & 24 & 36 & .187530 & 6 & 9 & 19 \\
\hline 14 & 3 & 17 & .235963 & 0 & 0 & 19 \\
\hline 15 & 2 & 44 & .299968 & 0 & 0 & 22 \\
\hline 16 & 1 & 25 & .389592 & 12 & 10 & 23 \\
\hline 17 & 6 & 15 & .480930 & 0 & 0 & 39 \\
\hline 18 & 29 & 34 & .583867 & 0 & 0 & 26 \\
\hline 19 & 3 & 24 & .696516 & 14 & 13 & 23 \\
\hline 20 & 33 & 45 & .809348 & 0 & 0 & 31 \\
\hline 21 & 7 & 35 & .923062 & 0 & 0 & 30 \\
\hline 22 & 2 & 11 & 1.072813 & 15 & 0 & 24 \\
\hline 23 & 1 & 3 & 1.259343 & 16 & 19 & 36 \\
\hline 24 & 2 & 18 & 1.452708 & 22 & 0 & 31 \\
\hline 25 & 10 & 21 & 1.665301 & 0 & 0 & 29 \\
\hline 26 & 29 & 30 & 1.883873 & 18 & 0 & 33 \\
\hline 27 & 8 & 14 & 2.212037 & 0 & 0 & 32 \\
\hline 28 & 16 & 19 & 2.614225 & 0 & 0 & 37 \\
\hline 29 & 9 & 10 & 3.053025 & 0 & 25 & 35 \\
\hline 30 & 7 & 38 & 3.524215 & 21 & 0 & 34 \\
\hline 31 & 2 & 33 & 3.998463 & 24 & 20 & 40 \\
\hline 32 & 8 & 28 & 4.504959 & 27 & 0 & 38 \\
\hline 33 & 5 & 29 & 5.024105 & 0 & 26 & 36 \\
\hline 34 & 7 & 13 & 5.877189 & 30 & 0 & 41 \\
\hline 35 & 9 & 20 & 6.749629 & 29 & 0 & 39 \\
\hline 36 & 1 & 5 & 7.838040 & 23 & 33 & 40 \\
\hline 37 & 4 & 16 & 8.943293 & 0 & 28 & 42 \\
\hline 38 & 8 & 12 & 10.054878 & 32 & 0 & 42 \\
\hline 39 & 6 & 9 & 11.458107 & 17 & 35 & 41 \\
\hline \% & 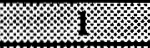 & 3 & $8 / 3 / 6 / 66$ & 6 & א & 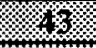 \\
\hline 4 & . & r. & 16058622 & 39 & 终 & (1) \\
\hline 42 & 4 & 8 & 21.180387 & 37 & 38 & 43 \\
\hline 43 & 1 & 4 & 27.448673 & 40 & 42 & 44 \\
\hline 44 & 1 & 6 & 35.812939 & 43 & 41 & $\mathbf{0}$ \\
\hline
\end{tabular}

Table XII: Agglomeration schedule for clustering of self-space labels 


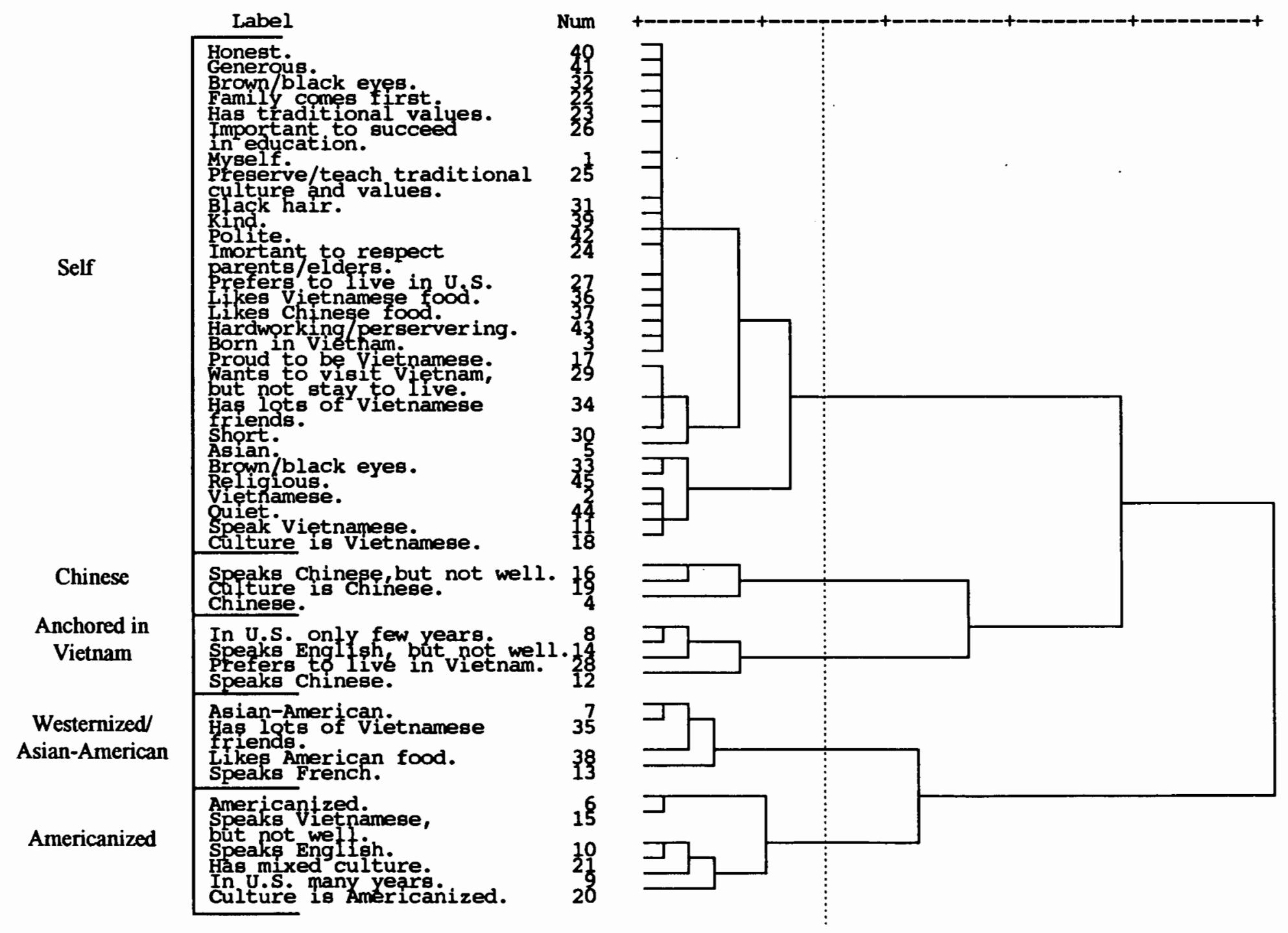

Figure 24: Dendogram of self map labels 
A meaningful jump in the distance coefficient was identified between stages 40 and 41 of the agglomeration process - highlighted in Table XII. Therefore, the process was stopped after stage 40 and a 5 cluster solution was chosen. Again, this is represented on the above dendogram by a broken vertical line. Based on their similarity to clusters in the previous analysis, the clusters were given summary names and superimposed onto the MDS configuration by drawing a boundary around each cluster of items. The combined configuration is presented in Figure 25.

In the map of the subjects' perceived social world, the self was represented as a unique point. Further, this point was positioned with those items that were most uniquely associated with self. In contrast, this map represented the self as the entire space within which the "Myself" point appeared as an idealized center of selforientation. Because one's social self is to a large extent influenced by the view one has of the social world (Duex, 1992), the social and self-salience spaces were generally expected to match. In large part, personal identity choices are generally defined by the social context and an individual's perceptions of those identity choices are affected by the meanings they have for the individual. However, other researchers have emphasized that the two needn't completely match in all respects (e.g., Weinreich, 1980). Often one's vision of self diverges in many ways from how one conceptualizes the external social world. During the sorting task, the subjects often expressed this perceived mismatch. After taking great care in distinguishing between different social groups, the subjects often 


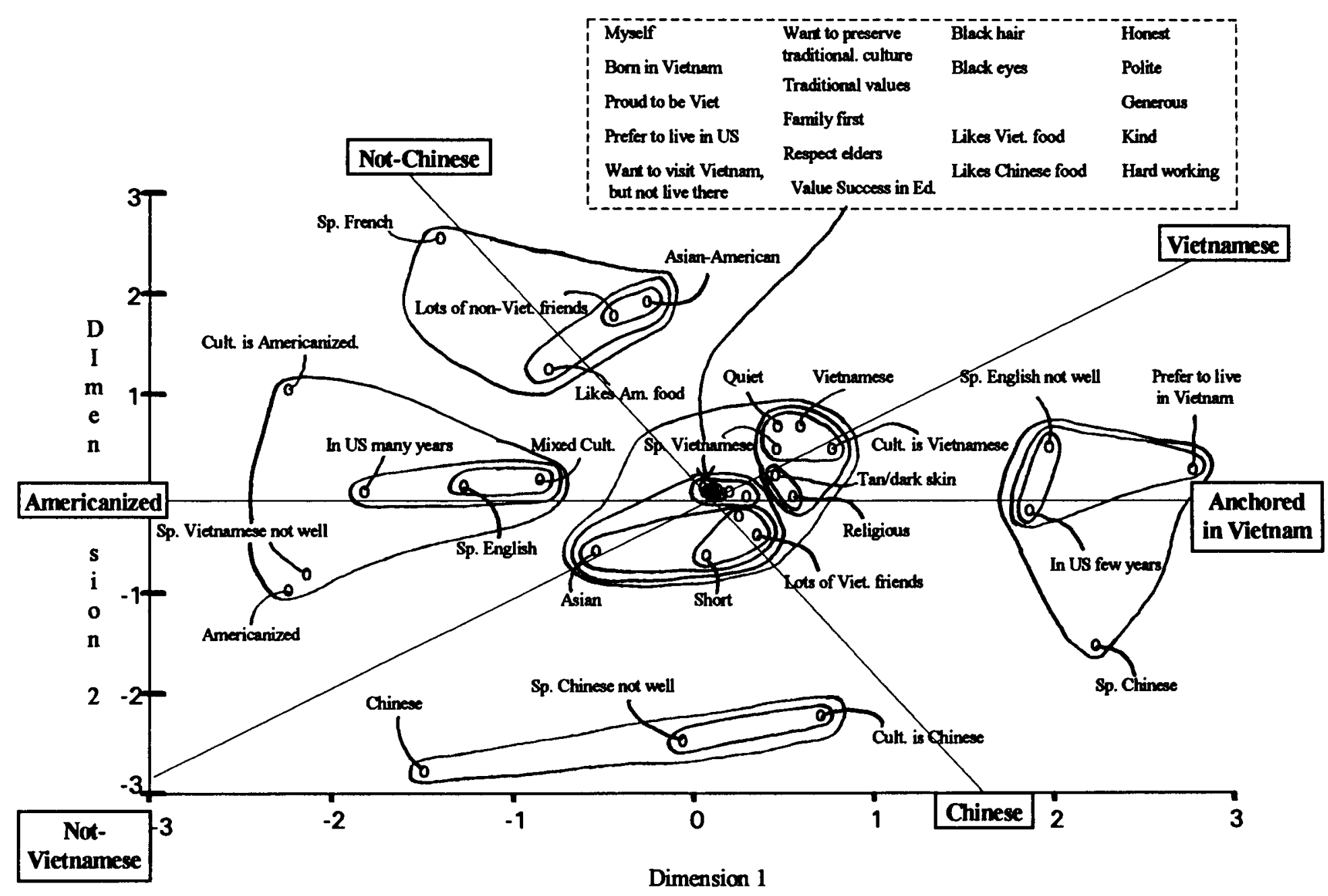

Figure 25: Self-salience map 
labeled one group simply "Myself" or "Me;" and many times they commented that both individually and as a peer group they were actually quite different from the all of the other types of people that they had represented in their sorting. That is, individuals thought of themselves as uniquely varying from the categories they pictured in their social world. However, as a group this uniqueness showed a high degree of consistency. The mapping of the "Myself' groups allowed me to look at the structure of this perceived uniqueness.

As expected, the basic structure of the self space was similar to that of the perceived social space. There were five clusters in the self-space which roughly corresponded to the clusters of the social space. Distributed along the periphery of the space were several clusters including, Chinese, Americanized, Westernized/Asian American, and Anchored-in-Vietnam clusters. Centrally placed in the space was a large cluster labeled "Self." This cluster had several sub-groups two of which were almost exactly the same as the two sub-groups which made up the Shared Characteristics cluster in the social map. In addition, the Self-cluster also contained a Vietnamese subcluster which was almost exactly the same as the Vietnamese cluster of the social map. Importantly, all of the labels which were centrally placed in the social map were still centrally placed in the self map. This indicated that the labels which were generally perceived as the shared characteristics for the community as a whole were the most self-salient aspects of the subjects' identity. 
There were a few very small differences in cluster membership between the social and self maps. In the self-space, the following labels moved from the Vietnamese to the most central Self-cluster: "Myself," Lots of Vietnamese friends," "Born in Vietnam," and "Proud to be Vietnamese." There were also a few labels that shifted into the Vietnamese sub-cluster. These included, "Tan/Dark skin," "Religious," and "Quiet." The Americanized cluster was basically the same as in the social space, though a few items from within the Americanized cluster of the social space formed an independent cluster within the self-space. "Likes American food" and "AsianAmerican," combined with "Lots of Non-Vietnamese friends" and "Speaks French" to create a Westernized/Asian-American cluster. Finally, all of the labels which comprised the Recent-immigrant cluster within the social map appeared together with "Speaks Chinese" as an Anchored-in-Vietnam cluster. Except for these few differences, the clusters of the social and self-spaces were very similar.

The vectors of the self-space were also very similar to those in the social space. Three meaningful vectors were identified. These included a Vietnamese/NotVietnamese vector, a Chinese oriented/Non Chinese oriented vector, and an Anchoredin-Vietnam/Americanized vector. Since the entire map was a self-salience space, the self-salience vector of the social map was not present. In addition, the clustering results suggested that in the self-space, the Long/short residency vector of the social space was subsumed by the Anchored-in-Vietnam/Americanized vector. Given the same basic clustering structure and the same basic vectors, the orientation of the labels 
within the space was also similar to that of the social map. However, in the self-space, the positions of the individual labels were re-oriented to reflect their relative importance in relation to "Myself."

In order to make the analysis of the self-salience space easier, the distances of each of the labels from "Myself" were entered into SPSS and submitted to a hierarchical clustering procedure using a Wards Euclidean distance model. The resulting dendogram appears below as Figure 26 . This process statistically divided the labels into concentrically-grouped levels of self-salience. Table XIII presents the labels ranked from most to least self-salient with the hierarchical clustering results indicated by the lines dividing the list. In addition, the last step in the hierarchical clustering results indicated an extreme jump in the level of self-salience. This major division in the level of self-salience was used to divide the labels into the two basic levels of core and peripheral self. Figure 27 presents this division superimposed onto the self-space.

The self-salience space provided a visual description of the subjects' identity. The group was most defined by those labels which represented the common characteristics of the subjects' perceived community. With a few exceptions, these core labels were not heavily loaded on any particular cultural vector in either the social or self-space. In general, these most core elements consisted of a preference for living in the US, a key set of family values, a perception of a shared physical appearance, a common set of food preferences (including Chinese and Vietnamese influences), and a 
Label

Has mixed culture Religious

Speaks English

culture is vietnames

Quiet

Vietnamese

Tan/dark skin

Short

Likes American food

Visit Vietnam not stay

Generous

Born in/from vietnam

Speaks Vietnamese

Proud to be Vietnamese

Lots of Vietnamese friend

Respect elders

Brown/black eyes

Polite

Hardworking/perservering

Family comes first

Kind

Honest

Likes Vietnamese food

Likes Chinese food

Prefers to live in US

Black hair

Has traditional values

Preserve trad. Culture

Myself

succeed in education

Asian-American

Speaks English not well

In US only few years

Lots of non-viet friends

In US many years

Chinese

Speaks French

Culture is Chinese

Culture is Americanized

Americanized

Speaks Chinese not well

Prefers to live in vietnam 28

Speaks Vietnamese not well 15

speaks Chinese

Num

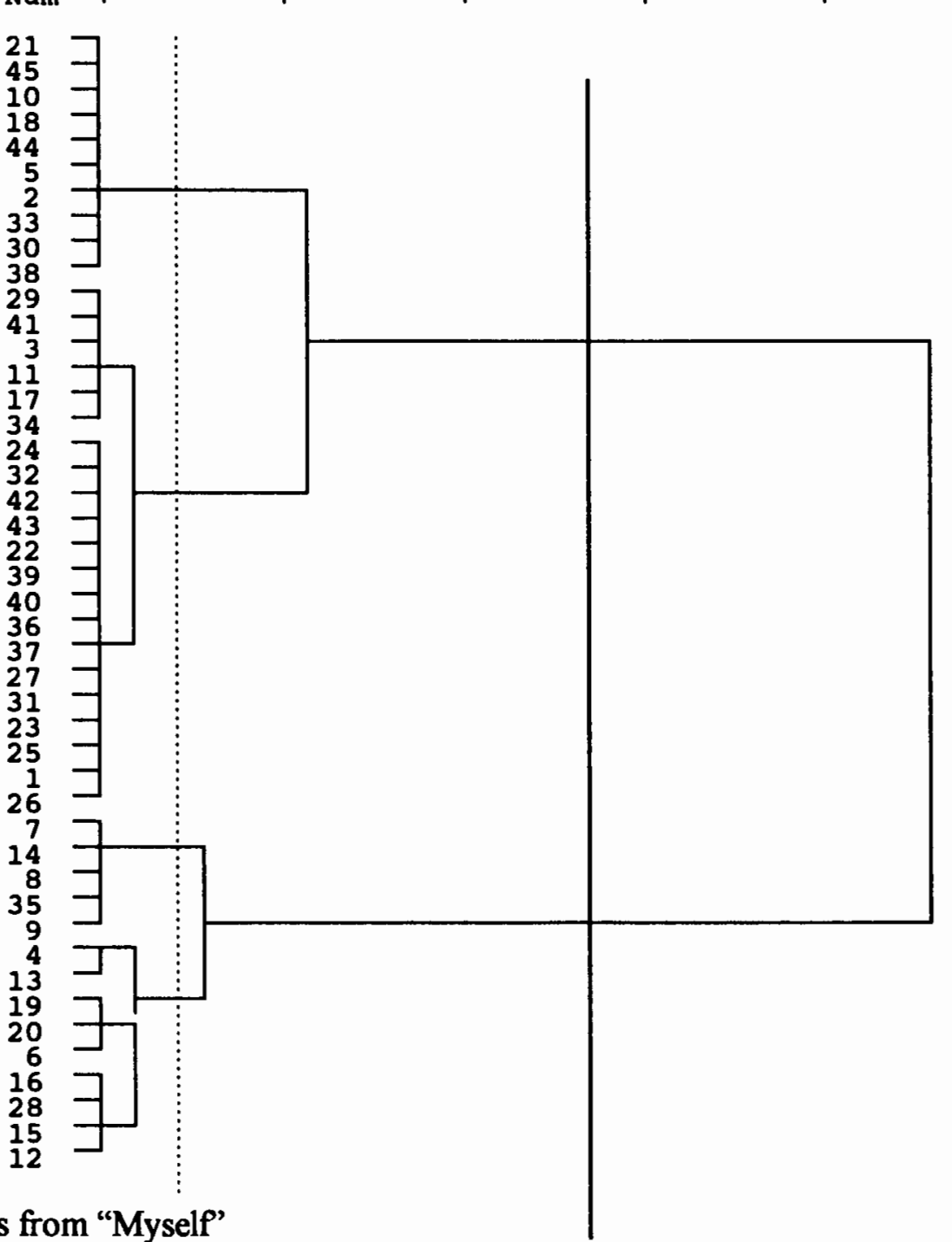

Figure 26: Dendogram of distances from "Myself" 


\begin{tabular}{|c|c|c|}
\hline Label & Distance & \multirow{32}{*}{ Core self } \\
\hline Myself & .00 & \\
\hline It's important to succeed in education & .04 & \\
\hline Brown/black eyes & .06 & \\
\hline It's important to respect parents/elders & .06 & \\
\hline Black hair & .07 & \\
\hline Family comes first & .07 & \\
\hline Hardworking/perservering & .07 & \\
\hline Has traditional values & .07 & \\
\hline Honest & .07 & \\
\hline Kind & .07 & \\
\hline Likes Chinese food & .07 & \\
\hline Likes Vietnamese food & .07 & \\
\hline Polite & .07 & \\
\hline Prefers to live in the US & .07 & \\
\hline wants to preserve traditional culture/values & .07 & \\
\hline Born in/from Vietnam & .21 & \\
\hline Generous & .21 & \\
\hline Speaks Vietnamese & .21 & \\
\hline Wants to visit Vietnam, but not stay & .21 & \\
\hline Has lots of Vietnamese friends & .24 & \\
\hline Proud to be Vietnamese & .24 & \\
\hline Tan/dark skin & .60 & \\
\hline Vietnamese & .60 & \\
\hline Asian & .68 & \\
\hline Culture is Vietnamese & .68 & \\
\hline Quiet & .68 & \\
\hline Has mixed culture & .73 & \\
\hline Religious & .73 & \\
\hline Speaks English & .73 & \\
\hline Likes American food & .90 & \\
\hline Short & .99 & \\
\hline Been in the US for many years & 1.24 & \multirow{14}{*}{ Peripheral Self } \\
\hline Been in the US only a few years & 1.50 & \\
\hline Has lots of non-Vietnamese friends & 1.50 & \\
\hline Asian-American & 1.67 & \\
\hline Speaks English, but not very well & 1.71 & \\
\hline Americanized & 2.03 & \\
\hline Culture is Americanized & 2.03 & \\
\hline Culture is Chinese & 2.03 & \\
\hline Prefers to live in Vietnam & 2.40 & \\
\hline Speaks Chinese, but not very well & 2.40 & \\
\hline Speaks Vietnamese, but not very well & 2.40 & \\
\hline Speaks Chinese & 2.44 & \\
\hline Chinese & 2.96 & \\
\hline Speaks French & 2.96 & \\
\hline
\end{tabular}

Table XIII: Ranked distances from "Myself" 


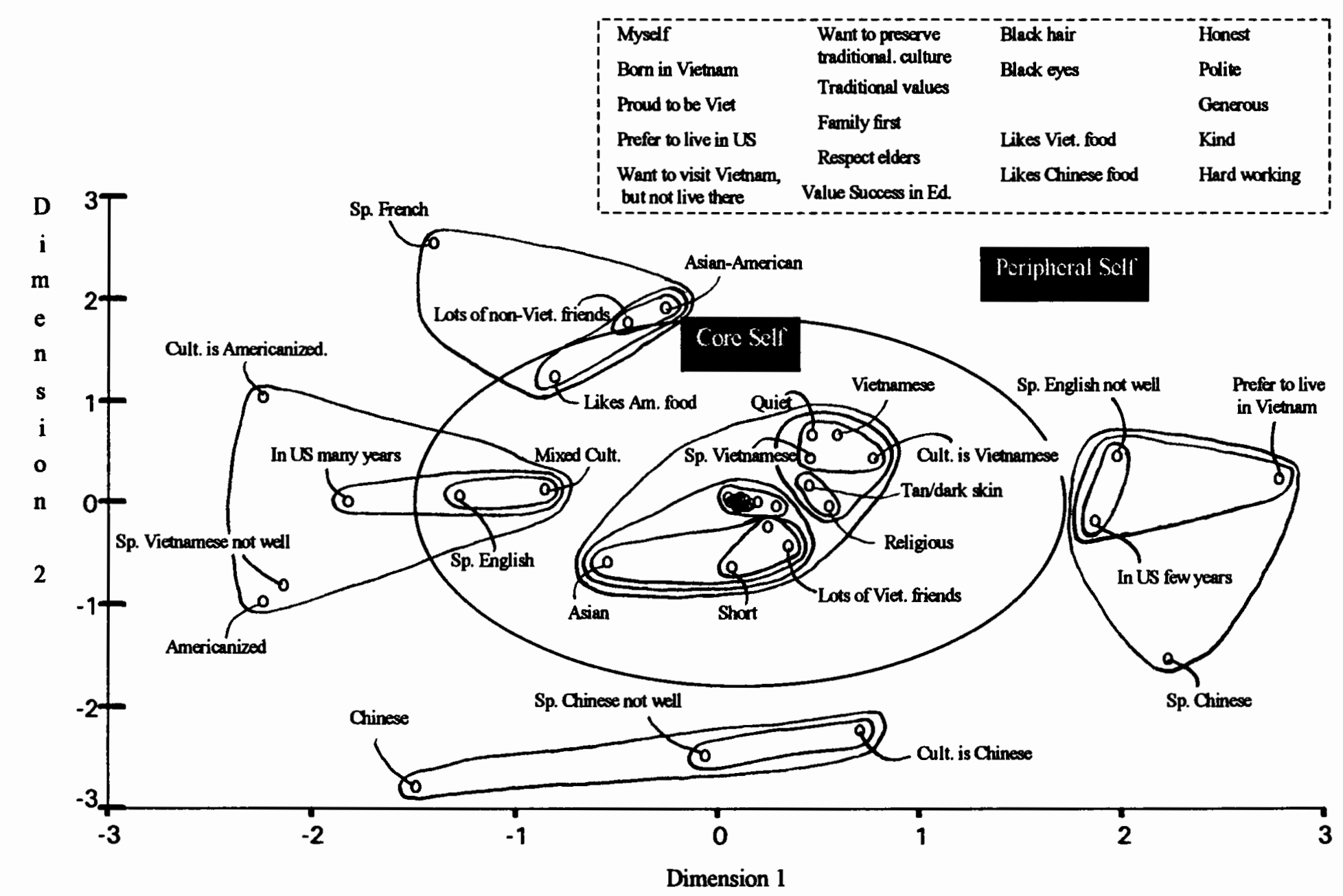

Figure 27: Self space divided into core self/peripheral self 
set of positive personal/group attributes. The exceptions included the labels, "Born in Vietnam," and "Proud to be Vietnamese." In the perceived social space, these were associated with Vietnamese-ness; however, in this map they could also be understood simply as a positive sense of shared historical origin. This would be consistent with previous research which has suggested that ethnic identification and pride is to a great extent independent from the maintenance of specific cultural knowledge or practices (e.g., Keefe \& Padilla, 1987; Laroche, Kim, Hui, Joy, 1996; Masuda, Matsumoto, \& Meredith, 1970).

Only slightly less self-salient was the sub-cluster of labels which was uniquely associated with Vietnamese group identity. This cluster included labels referring to Vietnamese culture and language, religion, and a few personal/group attributes. Also highly self-salient was the sub-cluster of items containing the identity label "Asian."

Less self-salient (though still within the core self area of the space) were three items from the Americanized end of the space. These were "Mixed culture," "Speaks English," and Likes American food."

Given this description, how can the linguistic identity of the group be characterized? In section 3.4 (pp.54-69), I presented three representations of possible schema structures related to bilingualism. They were monism (instrumentalism), bifurcation (diglossia), and transference (nativization). Each representation is duplicated below (p.156). In monism/instrumental bilingualism, each language was 
conceptualized as strongly associated with a distinct ethno-cultural identity, but only one culture/language system was a part of the user's identity.
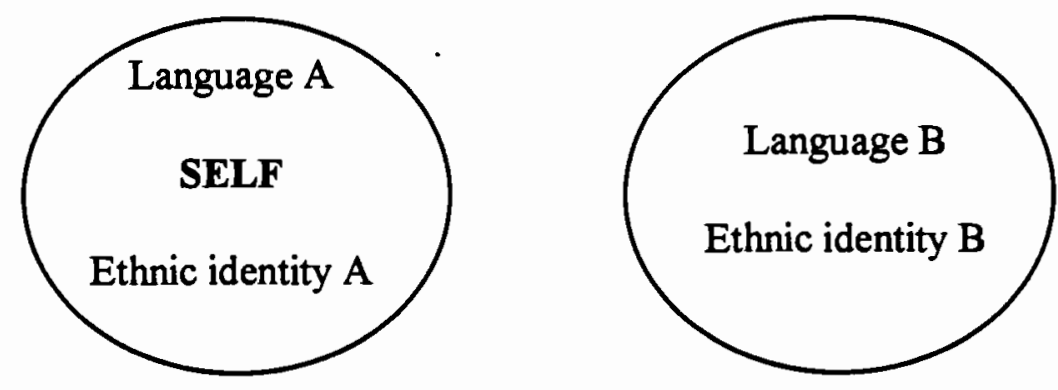

Figure 10. Schema structure of monism

In a state of bifurcation, each language was associated with a distinct ethnocultural identity and both culture/language systems were strongly established within the user's identity.

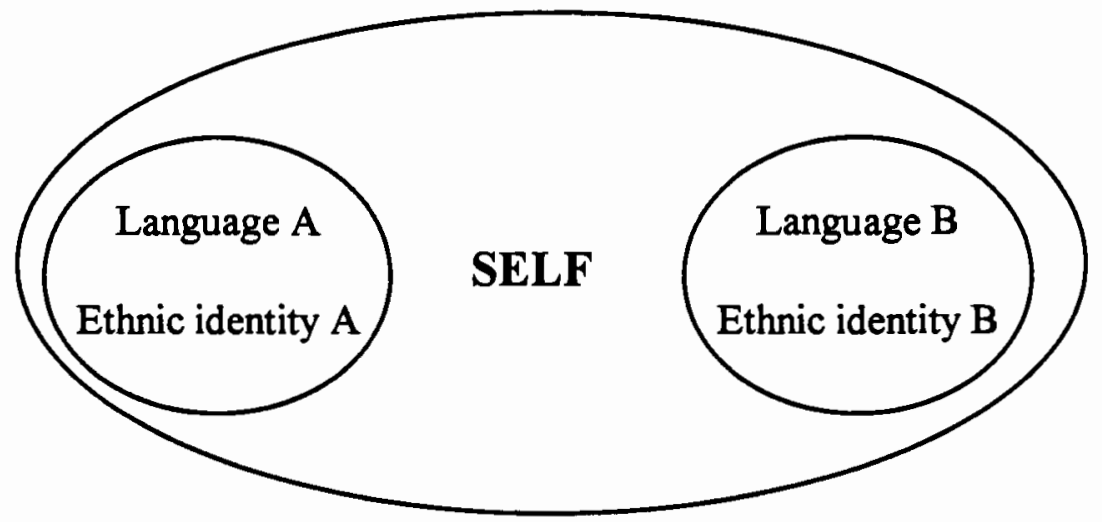

Figure 11. Schema structure of bifurcation

In a state of transference, the languages were not separated into dichotomized ethno-cultural identities; instead, both languages were associated with the user's native identity. 

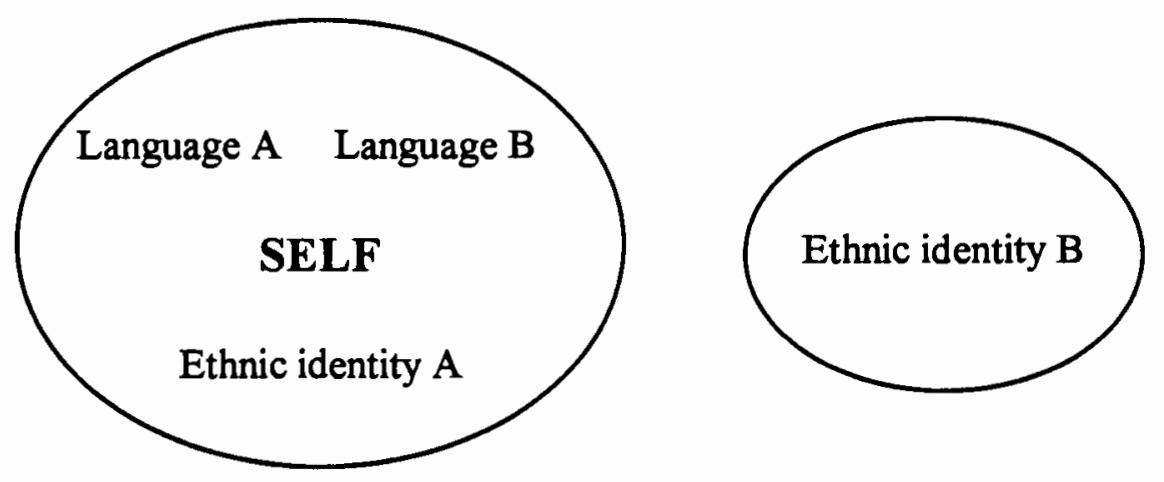

Figure 12. Schema structure of transference

The structure of the self-space was not easily categorized into a single model.

For example, the self-space had three separate culture/language clusters that were distinct from each other. However, only one of these culture/language clusters was highly self-salient within the space. Further, as a language, Vietnamese was clearly much more central to the subjects' identity than either Chinese or English. Therefore, ultimately, the identity structure did not conform to a bifurcation model.

Considering the amount of separation between English and most of the other Americanization labels and also that the label "Speaks English" was a part of core identity, the possibility of a transference model was considered. However, several other aspect of the map suggested that a transference model was inappropriate. First, English was not closely associated or clustered with Vietnamese identity. Second, English probably appeared too distant from either the self or the Vietnamese cluster to suggest transference. As a consequence, nativization would be unlikely and a transference model was rejected. 
In some respects, the group's identity structure conformed best to a monism/instrumental configuration. English, Vietnamese, and Chinese languages were grouped in respective cultural clusters and they were separated from one another along respective cultural vectors. However, an instrumental interpretation deserves some qualification. While Vietnamese language was tightly clustered with the other Vietnamese identity and culture labels, English appeared relatively distant from the Americanized identity and culture labels. This suggested that English was perceived as less culturally bound than either Vietnamese or Chinese. This interpretation was further supported by its clustering with the label "Mixed culture." Though English was clearly not associated with Vietnamese identity, it may have been perceived as less bound by specific cultural influences than the other languages in the space. In addition, English was a salient part of the group's identity.

These qualifications are important for several reasons. Traditionally, instrumental language use has not been associated with high self-salience of the instrumental language. However, these results may suggest that instrumental use may not imply a lack of self salience. It seems that this group has taken ownership over English however, it is an English which is perceived to be more culturally neutral than the other languages involved-a sort of intra-national version of English as an international language. It may be that there are qualitatively different categories of instrumental use, some being more relevant than others to a users identity. This raises several issues. For example, given this possibility, the assumption that self-salience (or ownership of English) is a distinguishing characteristic between the Outer and 
Expanding Circles would be questionable. It might not be the case that Expanding Circle users lack a feeling of ownership of English, but they feel they own a different kind of English from Outer Circle users. It may be that many Outer Circle users perceive the language as connected to indigenous cultural identity and that many Expanding Circle users perceive the language as culturally neutral part of their intercultural or international identities.

\subsection{Context specific self-salience}

The social and self-salience maps represented the group's generalized identity structure. That is, these spaces represented an initial set of expectations with which an individual comes to a situation. However, perceived context is also important in determining which aspects of identity are salient at any given time. The last research question addressed how the perceived context would affect the choice of which aspects of identity were most self-salient:

RQ6: Which domains were important to the subjects' ethnic and language identities? How did an awareness of these contexts affect the subjects' conceptualization of their identity options? Did some identities or aspects of identity become more or less salient when different domains were considered?

In order to address this issue, the following procedure was included in the study. After the subjects had sorted the first 24 labels in the card-sorting task, they were given the following list of sentences: 
1. How I am with my parents

2. How I am with my brothers and sisters

3. How I am with my friends

4. How I am at school

5. How I am at work

Subjects were then asked to identify the five labels that they felt were most important for describing themselves in each domain. Since this task was done as a part of the card-sorting process, the subject sample was identical to that described for the card sorting. The results of this process were used to address the last research question:

The frequencies of choice for each label were calculated for each context and the distribution of the frequencies was examined. Generally, there was a large decrease in the frequencies of those items which fewer than $1 / 3$ of the subjects had chosen. Therefore, only those labels which at least $1 / 3$ of the subjects chose for each context were selected for inspection. The labels which met this criterion along with the frequencies of occurrence for each context are presented in Tables XIV to XIX.

\begin{tabular}{|l|c|c|}
\hline \multicolumn{2}{|c|}{ L. How I am with my parents } \\
\hline Label & Frequency & Social space cluster \\
\hline Respect parents and eiders & 25 & $\begin{array}{c}\text { Shared } \\
\text { Characteristics }\end{array}$ \\
\hline Speak Vietnamese & 20 & Vietnamese \\
\hline Traditional Values & 20 & $\begin{array}{c}\text { Shared } \\
\text { Characteristics }\end{array}$ \\
\hline Family comes first & 14 & $\begin{array}{c}\text { Shared } \\
\text { Characteristics }\end{array}$ \\
\hline Culture is Vietnamese & 13 & Vietnamese \\
\hline Proud to be Vietnamese & 10 & Vietnamese \\
\hline
\end{tabular}

Table XIV: Label frequencies for parent context 


\begin{tabular}{|l|l|c|}
\hline \multicolumn{3}{|c|}{ 2. How I am with my brothers and sisters } \\
\hline \multicolumn{1}{|c|}{ Label } & Frequency & Cluster \\
\hline Speak Vietnamese & 20 & Vietnamese \\
\hline Respect Parents and elders & 16 & $\begin{array}{c}\text { Shared } \\
\text { Characteristics }\end{array}$ \\
\hline Speak English & 14 & Americanized \\
\hline Traditional Values & 13 & $\begin{array}{c}\text { Shared } \\
\text { Characteristics }\end{array}$ \\
\hline Family Comes first & 13 & $\begin{array}{c}\text { Shared } \\
\text { Characteristics }\end{array}$ \\
\hline
\end{tabular}

Table XV: Label frequencies for sibling context

\begin{tabular}{|l|l|c|}
\hline \multicolumn{3}{|c|}{ 3. How I am with my friends } \\
\hline \multicolumn{1}{|c|}{ Label } & Frequency & Cluster \\
\hline Speak English & 19 & Americanized \\
\hline Speak Vietnamese & 15 & Vietnamese \\
\hline Mixed culture & 12 & Americanized \\
\hline Proud to be Vietnamese & 11 & Vietnamese \\
\hline Traditional values & 10 & $\begin{array}{c}\text { Shared } \\
\text { Characteristics }\end{array}$ \\
\hline
\end{tabular}

Table XVI: Label frequencies for friendship context

\begin{tabular}{|l|l|c|}
\hline \multicolumn{3}{|c|}{ 4. How I am at school } \\
\hline \multicolumn{1}{|c|}{ Label } & Frequency & Cluster \\
\hline Speak English & 20 & Americanized \\
\hline Mixed culture & 15 & Americanized \\
\hline Americanized & 12 & Americanized \\
\hline Speak Vietnamese & 12 & Vietnamese \\
\hline Proud to be Vietnamese & 11 & Vietnamese \\
\hline Respect Parents and elders & 10 & $\begin{array}{c}\text { Shared } \\
\text { Characteristics }\end{array}$ \\
\hline
\end{tabular}

Table XVII: Label frequencies for school context 


\begin{tabular}{|l|l|c|}
\hline \multicolumn{3}{|c|}{ 6. How I am at work } \\
\hline \multicolumn{1}{|c|}{ Label } & Frequency & Cluster \\
\hline Speak English & 20 & Americanized \\
\hline mixed culture & 14 & Americanized \\
\hline Respect elders and parents & 13 & $\begin{array}{c}\text { Shared } \\
\text { Characteristics }\end{array}$ \\
\hline Proud to be Vietnamese & 11 & Vietnamese \\
\hline Asian-American & 10 & Americanized \\
\hline Traditional Values & 10 & Asian \\
\hline
\end{tabular}

Table XVIII: Label frequencies for work context

\begin{tabular}{|l|c|c|}
\hline \multicolumn{1}{|c|}{ Label } & Frequency & Cluster \\
\hline Speak English 4/5 & 73 & Americanized \\
\hline Speak Vietnamese 4/5 & 67 & Vietnamese \\
\hline Respect elders and parents 4/5 & 64 & $\begin{array}{c}\text { Shared } \\
\text { Characteristics }\end{array}$ \\
\hline Traditional Values 4/5 & 43 & $\begin{array}{c}\text { Shared } \\
\text { Characteristics }\end{array}$ \\
\hline Proud to be Vietnamese 4/5 & 43 & Vietnamese \\
\hline Family comes first 2/5 & 27 & $\begin{array}{c}\text { Shared } \\
\text { Characteristics }\end{array}$ \\
\hline Mixed culture 2/5 & 27 & Americanized \\
\hline Culture is Vietnamese 1/5 & 13 & Vietnamese \\
\hline Americanized 1/5 & 12 & Americanized \\
\hline Asian-American 1/5 & 10 & Americanized \\
\hline
\end{tabular}

Table XIX: Frequencies of labels across all contexts.

Across all contexts, English and Vietnamese languages were the most frequently chosen items. The results showed that both English and Vietnamese were chosen for all but two contexts. The only Vietnamese monolingual context was "How I am with my parents" and the only English monolingual context was "How I am at work." Except for these two, bilingualism seemed to be a relatively stable 
characteristic across the majority of the contexts which were considered. On the one hand, the subjects may have perceived their parents to be more situated than themselves in Vietnamese monolingual contexts; and on the other hand, work contexts may have been perceived as having more emphasis on relationships with out-group members with whom English is the only shared language. The contexts which emphasized bilingualism were generally those in which subjects had access to in-group peers. This also seemed to extend to the family contexts. While the parent context was monolingual in Vietnamese, both languages were frequently chosen in the context of siblings.

The next most frequently chosen set of labels included "Respect for parents/elders," "Has traditional values," "Proud to be Vietnamese," and "Family comes first." All of these items appeared in the most core self-cluster in the selfsalience space. The first three of these labels appeared in 4 out of 5 of the contexts and were also within the Shared Characteristics cluster within the social space. That these items appeared in the most central parts of the identity spaces and were also consistently chosen as important for so many contexts suggested that they were relatively central and stable aspects of identity.

The least frequently chosen items were "Culture is Vietnamese," "Americanized," and "Asian-American." The Vietnamese culture label appeared only in the context of parents. The "Americanized" and "Asian-American" labels appeared only in the school and work contexts. 
The distribution of items among the contexts suggests several possible interpretations. Initially, the labels seemed to be arranged on a continuum from private to public with Vietnamese identity labels being more common within the private domains and Americanized labels being more common as the context becomes more public (e.g., school and work). However, the emphasis on the public/private distinction becomes less important when one considers the presence of peer group. Even in the privacy of the family, the sibling context showed less emphasis on uniquely Vietnamese labels. In general, the more important distinction seemed to be between those contexts that involved access to in-group peers and those that did not. The contexts that emphasized siblings, friends, and to some extent, school showed higher frequencies of labels which were associated with a more generally Asian, mixed, and bilingual identity. This would suggest that the group did perceive themselves as distinct from both their parents and from the more general society.

However, the subjects not only shifted which aspects of their identity were most salient along a Vietnamese/Americanized continuum, but also along a continuum of generality and mixed-ness. That is, as the group moved into perceived peer contexts there was more of an emphasis on the shared aspects of Asian identity and labels expressing mixed cultural influences and Vietnamese/English bilingualism.

\subsection{General discussion and implications}

Given the above results, the following general characterizations can be made about the group's linguistic and ethno-cultural identity. First, English was not 
associated with Vietnamese identity. Second, Vietnamese was most closely associated with the subjects' identity, though English was also a highly self-salient feature of that identity. Third, though Chinese culture and language were distinct influences within the subjects' social world, they were not core aspects of identity. Fourth, Americanization and the role of English seemed to have specially ambiguous meanings within both the structure of the subjects' perceived social world and the subjects' identity. On the one hand, several key Americanized identity labels were perceived to be in opposition to both Vietnamese culture and language. This part of Americanization was not a highly salient part of the group's identity. On the other hand, the concept of Americanization also had an aspect which was associated with a mixing of cultural influences. This aspect of Americanization was a highly salient part of the subjects' identities and it was this aspect with which English was most closely associated.

The perceived importance of mixing cultural influences was reported by Woon (1985) and also Wolfram et al., (1986). Both authors commented that their subjects generally felt a strong desire to blend together what was best from their past and current cultural influences. However, their results and also mine suggested that the perceptions of cultural mixing was highly structured and selective.

Within the identity space, the importance of the labels "English" and "Mixed culture" was given special meaning by their proximity to the most core aspects of the self-salience space. Beyond a few labels related to a sense of common group origin 
within Vietnam, the most core identity cluster within the space consisted of a set of more generally Asian characteristics which were perceived as common to the community as a whole. Though strongly self-salient, the cluster of labels related to Vietnamese identity was perceived as distinct from the most core self cluster. All of this suggested that while the space contained a strong Vietnamese influence, a central aspect of the group's identity may have been a more mixed and abstract sense of Asian or pan-Asian identity.

These findings point to a delicate balancing of identity maintenance and acculturation that was far more subtle than the conceptualization which originally informed the research questions. I had posed my research questions assuming that there would be a clear identification with either Vietnamese or Americanized identity, or possibly both. However, the results did not present either such a global or dichotomized picture of identity. On the one hand, the Vietnamese cluster (including Vietnamese language) was clearly important to the group's identity; however, on the other hand, the Vietnamese cluster was not the most important aspect of identity. Similarly, the group's identity was neither oriented strongly towards being anchored in Vietnam, nor was it strongly oriented towards Americanization. The strongest center of identity represented a basic sense of shared Asian appearance, Confucian family values, a preference for living in the US, and a number of positive personal/group attributes. This emphasis on more general Asian characteristics was consistent with Woon's (1985) study of Sino-Vietnamese in Canada. As Woon described: “Although 
some have anglicized their names and believe that they should learn from Canadians such external behaviour traits as frankness and independence, as well as Western etiquette and manners, they emphatically hope that they and their descendants retain such Confucian family virtues as respect for parents and the elderly, being good parents, and raising good children ... [and] convictions such as these cut across regional and socio-economic class origins" (Woon, 1985 p.544). Further, Woon argued that the emphasis on family, respect to elders, education and hard work were generally viewed very positively by the larger Anglo context. It may be that this emphasis on the more generally Asian aspects of the group's identity provides the most positive sense of identification across the widest range of social contexts. As such, this emphasis may be the most flexible balance between the pressures of identity maintenance and acculturation.

The suggestion that the group's identity was most anchored in a more abstract, or inclusive, level of identity points to a potentially important aspect of acculturation and identity maintenance which I have not seen discussed in the research literature. Acculturation may involve more than shifts and balances between specific ethnocultural influences (Vietnamese, Chinese, etc.); it may also involve changes in the very level of group identity that is most salient. Individuals may not only shift between specific cultural anchors during acculturation (Chinese to Americanized culture), but also shift among various levels of inclusiveness (Vietnamese vs. Asian). Several aspects of the present results would suggest this interpretation for the group. First, the 
most core sub-cluster of identity within the self-salience space matched the cluster of socially shared characteristics within the perceived social space. That is, the most core aspects of the groups' identity were also those which were thought of as the most socially inclusive. Second, this interpretation is consistent with the structure of the clustering results. The sub-cluster which was most core to identity was grouped first with the cluster including the label "Asian;" and only after this were both of these clusters merged with the Vietnamese cluster. Lastly, the labels from the core cluster of shared characteristics were generally salient within all contexts and most salient within contexts emphasizing peer group. All of this would suggest that these labels were associated with a more inclusive level of identity which was perceived as characteristic of the group.

By adding a continuum of abstraction to the spatial representation used in the MDS configurations, the identity/acculturation process can be represented as a conical model (Figure 28).

On this model, specific cultural influences appear as a surface within an identity landscape. This would be similar to the actual MDS space which formed the results of the study. In addition, one could add an elevation, which represents more inclusive levels of group identity. Specific labels would be distributed within this landscape along specific cultural vectors and also distributed along the elevation of the cone according to the perceived level of inclusiveness, or generality. 
Increasingly inclusive

levels of identity

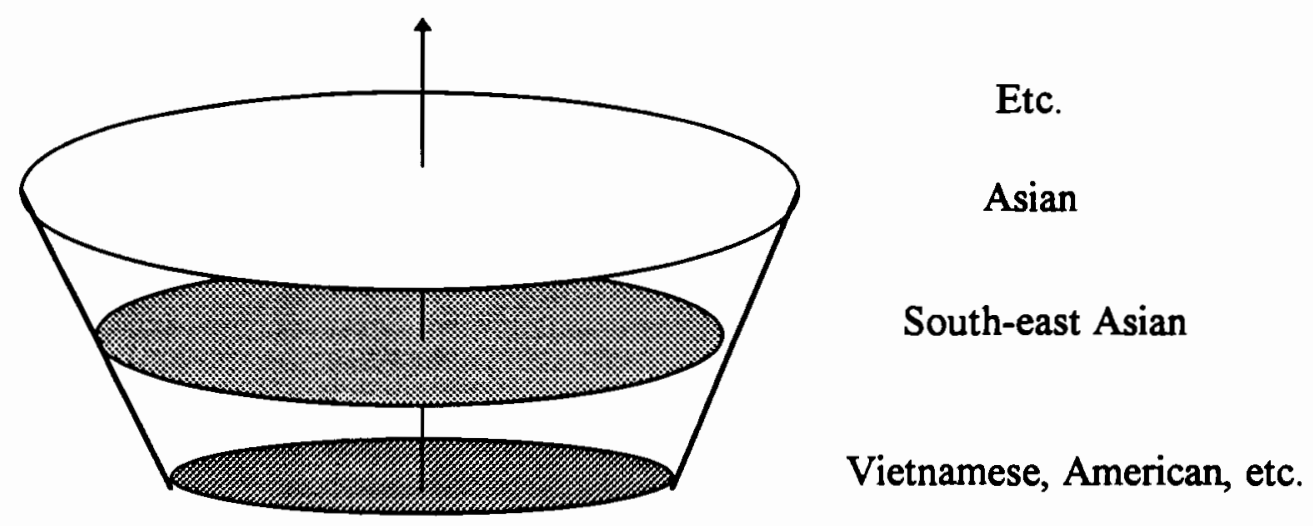

Culturally specific

levels of identity

Figure 28: Conical model of acculturation

For this particular group, the cluster of labels which were uniquely associated with specific ethno-cultural identities would appear on the base while the labels representing the cluster of shared characteristics would appear on a more abstract level of identity. Of course, the range of generality which is represented is arbitrary. There may be some elements within social identity that are also perceived as more compatible with interpersonal or individual levels of identity. Most probably the labels which I have referred to as personal/group attributes would fall in this group. Further, the individual items may be generally spread through-out the space, being more or less associated with one or more levels of identity just as they are associated more or less with various cultural influences. For example, within my results, Chinese language 
appeared to be highly culturally specific, whereas English seems to be perceived as more culturally general.

A number of researchers within social identity theory (e.g., Brewer, 1991; Brewer \& Schneider, 1990) have offered a theory of the interplay between self and context which is consistent with both the overall conceptualization and results of the present study. Within optimal distinctiveness theory, the self is conceptualized as a tensioned interplay "between needs for validation and similarity to others and needs for uniqueness and individuation (Banaji \& Prentice, 1994, p. 313). The theory was generated out of evidence that when individuals identify at levels that are either too unique or too undifferentiated for a given context, they tend to feel uncomfortable and as a result shift their identification. Brewer (1991) suggested that this happens because in either extreme case one's sense of security and self-worth becomes threatened. On the one hand, being too distinctive (even in positively valued characteristics) "leaves individuals vulnerable to isolation and stigmatization" (Brewer, 1991, p. 478). On the other hand, being too indistinct leaves individuals without any basis for self-definition and appraisal. Therefore, "social identity is a compromise between assimilation towards others (i.e., those in the in-group) and differentiation away from others (i.e., those in the out-group). Among the theory's main assumptions is that individuals will tend to identify with groups that best resolve this conflict between assimilation and differentiation" (Banaji \& Prentice, 1994, p. 313). This is exactly what one sees in the results of the present study. The group's identity seemed to be most strongly anchored 
in those elements which were a balance between group distinctiveness and inclusiveness. This is certainly true of the most core self cluster. However, this can also be true of the space in general. One can understand the self-space as being an area of influences within which one moves according to changes in the context. All aspects of the space are open to being salient given the right contextual influences. However, the periphery of the space tends to be generally less salient. Optimal distinctiveness theory provides a framework for understanding why the items are arranged in the space in this manner. Overall, those items that provide the most flexible balance within the overall context will tend to be the most core aspects of identity. That is, identity tends to be focused on that level which provides the optimum of both inclusiveness and individuation. For this group, optimal distinctiveness means balancing both the relationship with the wider social context and potential in-group differences. The items on the periphery of the space represent the most distinctive level of identity. Because they are potentially perceived as the most unique and least flexible anchors, they tend to be generally least self-salient, though the needs of the context may over-ride this at particular times. On this interpretation, the strong self-salience of labels along the Vietnamese vector would suggest that the overall context in which the group lives is generally Vietnamese in quality. However, the common Asian aspects of that identity have taken on a central importance for the group.

Optimal distinctiveness theory raises a number of important questions for the language issues in the study. Can the identity model provided by the MDS results be 
used to understand the patterns of language use for the group over time (i.e., language maintenance or loss, patterns of bilingualism, etc.)? Can it be used to understand micro-level patterns of code mixing, code-switching, borrowing, etc.,? A more detailed discussion of these issues is not possible here because they go far beyond the scope of the present study. The most that can be said is that the results point to vast areas of research that are yet to be done. 


\subsection{Limitations and Directions for Future Research}

Since most of the limitations of the present study are directly relevant to suggestions for future research, I will discuss them both together. The first set of limitations relates to the production and selection of the labels. First, several researchers have raised questions about the use of the TST. While one advantage of the TST is that the task is very open-ended, extremely open-ended tasks may not be appropriate for all cultural groups. In particular, cultures which are characterized by high uncertainty avoidance and/or low self-disclosure do not seem to perform well on the TST.(Cousins, 1989; Markus \& Kitayama, 1991,1992). Markus and Kitayama (1991,1992, 1994), in particular, have argued that subjects with an inter-dependent self-construal depend heavily on their perceived role relations to define themselves. Therefore, they may not be able to provide self-descriptions unless the context is very specific. Given these tendencies, some cultural groups may not supply all of the information which is actually self-relevant when faced with a highly open-ended and decontextualized task such as the TST. Further, subjects with an inter-dependent selfconstrual may be more apt to interpret the TST in relation to their role as a research subject and adjust their answers more to what the researcher seems to want than what they actually think about themselves. It may be that more naturalistic methodologies, such as informal interviews or participant observation, may yield different and more valid results for these groups. The present study addressed these issues by forming relationships with the subjects through informal friendship networks and also by 
contextualizing the task in an imaginary pen pal situation. However, there is very little research about the how Vietnamese populations score on uncertainty avoidance, selfconstrual, etc. Further, there are not any studies that I am aware of which address these issues for Vietnamese Americans as a separate group. Further work definitely needs to be done in this area.

Limitations in the use of the TST would suggest that the present results should be confirmed through multiple methodologies. The TST results could be confirmed either by triangulation with more naturalistic methodologies or more guided follow-up tasks (e.g., ranking on the basis of perceived self descriptive-ness, Likert scale judgments of self-descriptive-ness, etc.,).

A second set of possible limitations was related to the process of reducing the TST data to an appropriate number of labels for the card-sorting task. The TST stage produced over 1200 self-descriptions. However, the card-sorting task required a maximum of 40 to 50 labels (and this number was usable only by staging the sorting process). The data reduction was accomplished through an inclusion criterion and a semantic grouping process. Only those items that appeared on more than one TST and were produced by more than one subject were included in the semantic grouping procedure. This seemed a reasonable minimum requirement. However, this also meant that several items may have been either included or excluded based on the amount of data that was collected. If a fewer number of subjects had been used, several items which were included may not have been selected and if a greater number of subjects 
had been used, more items would have most probably been included. Because there is no a priori rule for determining the appropriate number of subjects in this case, it's unclear how to solve this problem. Stopped data collection at the point when it appeared that additional data collection merely produced redundant results. However, there is always the possibility that important data were left out.

Once the data had been reduced through the selection criteria, the semantic grouping process presented another set of limitations. In particular, deciding what constituted the "same meaning" was not a mechanical process. Though many of the items were exact, or very closely exact matches, a large number of descriptions had to be grouped together based on my perception of their similarity. I made these judgments after a large amount of informal input from several different sources, including experts in the fields of psychology, linguistics, and inter-cultural communication. In addition, I also solicited the views of natives of VietnameseAmerican culture and a few members of the actual subject population. Because the main concern of the data reduction was to produce a list of labels which represented the overall diversity of meanings in the original TST results, I chose not to be overly concerned about the validity of how individual descriptions were categorized. That is, differences in how individual items could be categorized did not have to be big concern as long as long as the same categories were produced as an end product. As a consequence, I did not concern myself with formalized grouping procedures and intercoder agreement statistics (e.g., Kappa statistics). However, this still leaves the 
question open whether the same set of categories would be produced by a more formal semantic grouping procedure. While my main concern was overinclusive-ness, a more formal procedure may be useful for eliminating superfluous labels. There were a few items (e.g., "Speaks French") which had very low levels of co-occurrence with all of the other labels and may have been irrelevant. This concern becomes important because superfluous items may affect the final MDS configuration in unknown and unwanted ways. In addition, a more formal semantic grouping procedure could allow one to analyze the self-descriptions as qualitative data. A formal analysis of the rich qualitative data in the TST results would certainly be another direction for further research withilout the need for further data collection.

As in any study involving different languages, the translations should be further confirmed. In the case of the present study, the concern was not only with the translations per se, but also with the effect of language choice in general. Since the group was highly bilingual, it seemed reasonable to allow each subject to have access to each language throughout the task. However, it is unclear how choice of language and/or access to both languages may have affected the final results. Further research into the relationship between language and task performance is important not only because different languages may produce different results, but also because limiting a bilingual population to one language may also bias the results of any study.

Within the MDS stage, the main limitation was the subjective nature of the interpretation process. I used a subjective interpretation approach. As with the 
semantic grouping process, I used a large amount of informal input from both active researchers and cultural natives. However, both outside experts and natives have their own set of biases and tend to see what they want in the results. Neither being an expert in the field or a member of the subject population assures the validity of an interpretation. Therefore, the present interpretations need to be taken very cautiously. A formal confirmation of the vectors and their interpretations needs to be carried out. A confirmation can be done by selecting an appropriate set of the labels and having either the same subjects, or a reasonably similar group, judge the labels on the various criteria which the vectors represented. One possibility would be to use Likert-type scales and then analyze the data with multiple regression techniques. Another possibility is to use the labels for a separate factor-analytic conformation of the vectors. If the results of these independent procedures match the MDS results, then the interpretations are confirmed and the implications of the findings can be considered with greater certainty.

The biggest limitation of the study was the sampling procedure. The population for the study was a relatively small (42 subjects for the TST and 30 subjects for the card sort). In addition, the population was not randomly chosen.. Therefore, the generalizability of the results is very limited. This would suggest that even though many of the results were consistent with previous studies, they should be taken very cautiously. Again, replication is recommended. If a much larger sample is accessible, a potentially fruitful aim would be to study the effect of key demographic variables 
(socio-class, time in country, level of education, level of proficiency in the languages involved, etc.,). Another potential area for exploration would be relating sub-group differences to more formal linguistic data (the presence of unique linguistic markers, discourse patterns, patterns of code switching, etc.,). Any of these areas are open to further exploration given access to an appropriately chosen and large enough population. 


\title{
Notes
}

\begin{abstract}
${ }^{1}$ The concepts of racial, ethnic, and cultural identity have a variety of definitions and uses. At this point, I will leave their definitions rather vague and opt to use both "ethnic" and "ethno-cultural" interchangeably. An extended discussion of identity issues will be given in section 3.0.
\end{abstract}

${ }^{2}$ Because it is the politically most inclusive term, I will use "Vietnamese-American" throughout this text. However, how individuals in this group actually feel about themselves (e.g., VietnameseAmerican vs. Vietnamese living in the US vs. American, etc.) is an empirical question.

${ }^{3}$ For a discussion of the concept of pluricentricity and related terms see Kachru (1990, pp.159-160; footnotes, p. 171).

${ }^{4}$ The importance of this secondary migration is consistent with Tran's (1987) report that level of social support was the most important predictor of psychological stress for Vietnamese immigrants-English language ability for the most part had no notable direct effects on alienation.

${ }^{5}$ The authors state: "Because of positive attitudes toward Vietnamese and other factors contributing to it's maintenance, the language may be maintained longer in this community than has been the case in other immigrant situations (Wolfram, et al., 1986, p. 53). It seems that the authors expect that Vietnamese will cease to be maintained at some time in the future, though it may "hang on" an unusually long time. This comment may be meant merely as a matter of fact prediction based on past cases. However, it also seems that the objective tone and the choice to describe the process as one inherent in the Vietnamese community belies the real social forces which are at work to promote Vietnamese language attrition.

${ }^{6}$ It is important to note that Wolfram et al.'s (1986) approach is in the tradition of what Mohanan (1992) has described as a parasitic approach. That is, VE is seen as parasitic on mainstream standard American English; therefore, it is described as "lacking" features of its host (e.g., it lacks final stops). 
This is done because Wolfram et al. argue that VE is an interlanguage with standard American English as its model and they present qualitative and quantitative data to support the position that Vietnamese have chosen standard English as an acquisitional target.

${ }^{7}$ Several authors present detailed descriptions of this view of culture (e.g., Nishida, 1989; Shimanoff, 1980; Littlejohn, 1989).

${ }^{8}$ Kachru has presented several graphical versions of this same model. I have adapted the version from Kachru (1989). For sake of brevity, I have deleted the population figures that appeared in the original and summarized the estimates of total users. In addition, I have added Vietnam to the list of Expanding Circle countries in accordance with Denham's (1992) arguments. This version is one of the more conservative lists of Outer Circle countries. For example, Lowenburg (1984) presents a more extensive list which also includes Botswana, Burma, Cameroon, Ethiopia, Fiji, Gambia, Israel, Lesotho, Liberia, Malawi, Malta, Mauritius, Namibia, Sierra Leone, South Africa, Sudan, Swaziland, Tanzania, Tonga, Uganda, Western Samoa, Zimbabwe.

${ }^{9}$ More recently these designations have been questioned--in particular the label of Outer Circle countries as ESL countries. For many people in these countries English is clearly a native language; and for many others English is learned as early, used as much, and is as important as the other languages available to them. Because of this, the application of many traditional dichotomies such as native / non-native, first / second, etc. should be used with caution. Similarly some authors (Kachru, 1990; Kahane, 1982) have pointed out that nativization is not unique to the Outer Circle. It was an important influence in both American and Australian Englishes and was resisted by the British colonial center in much the same way as it is being resisted by today's centers of English colonialism. ${ }^{10}$ As several authors (e.g., Phillipson, 1992; Pennycook, 1994) have pointed out, the line between imperialism and development is not very clear and in many cases the two phenomena are identical. In 
fact, some authors (e.g., Phillipson, 1992) seem to simply equate "Inner" and "Outer" with "Center" and "Periphery" in theories of development and colonialism.

${ }^{11}$ Though it has been common to refer to these varieties of English as "nonnative varieties,"--usually for the simple reason that they are not Inner Circle varieties--one should remember that many of these speakers can be considered "native" speakers as much as any one in Great Britain, or the United States (see Paikeday, 1985; Phillipson, 1992, pp. 160-164; Kubchandani, 1983; and Davies, 1991 for appropriate discussions of the concept of "native speaker." Also see footnote number 24.

${ }^{12}$ Though I haven't read any explicit statement to this effect in the literature, it seems that an attitude of ownership over English is one of the most important distinguishing characteristics between Outer and Expanding Circle countries.

${ }^{13}$ See Pride's (1983) discussion of African authors who feel that "English can, and does, convey something of what being a West African, Kikuyu, West Bengali, even Black, etc. exactly means" (notes)

14 The term "critical linguistics" refers to an approach to language issues informed primarily by work in cultural studies, critical theory, dependency theory, etc.

${ }^{15}$ Both Phillipson (1992) and Dasgupta (1993) suggest this in recent works. Phillipson seems to suggest that speakers of institutional varieties of English could only be surrogate colonial elites. Dasgupta argues very openly that since the meaning of English in India is dependent on the meaning of English in its native habitat (p. 49), its use within India is culturally sterile. According to Dasgupta, English in India can not be more than a conduit for Western values promoted by a small elite that "only emulate" but can never be either Indian or Western. 
${ }^{16}$ This attitude is also becoming apparent through the increasing absence of arguments and preambles which attempt to justify the status of specific varieties studied by Outer Circle authors (e.g., KwanTerry, 1991; Pakir, 1993). Fernando (1986) has argued that one sees this new attitude in the Outer Circle because Inner Circle language authority has now dissipated with "the colonial momentum under which it had thrived" (p. 197). Fernando simply proclaims that within many Asian countries, decisions about the role, status, and character of English in those countries should now be guided by "deliberate choice on the part of Asians" (p. 197; my emphasis).

${ }^{17}$ More recently, this was also supported in both Markus and Wurfs (1987) review of the subject and Banaji and Prentice's (1994) review of psychological literature on the self since 1987. As they state:

If the number of papers published on a topic is an accurate indicator of the interest it evokes and the attention it commands, then the self continues to hold center-stage position in psychology. Over $\mathbf{5 0 0 0}$ articles about the self have been published since the last Annual Review of Psychology chapter on the topic appeared seven years ago ....

(Banaji \& Prentice 1994, p. 297-98)

${ }^{18}$ This should not be confused with the hypothesis that there is a causal direction of influence between the constructs of self and culture. It is merely meant as a conceptual hierarchy useful for understanding the relationship between the construct of self and the phenomena of cultural variations in individual experience and behavior.

${ }^{19}$ Following up on Markus and Kitayama, several other researchers have begun to develop measures of independence versus interdependence (e.g., Cousins, 1989; Singelis, 1994), and interdependent oriented measures of self esteem (e.g., Luhtanen \& Crocker, 1991 \& 1992; Crocker, Luhtanen, Blain \& Broadnax, 1994; for a summary of issues in collective identification and self esteem see Banaji \& Prentice, 1994, pp. 310-313).

${ }^{20} \mathrm{Kachru}(1995)$ questions both interlanguage theory and notions of the cultural unity of English.. Kubchandani (1983) questions the use of the concepts of a native speaker, a mother tongue, and the 
analytic approach of scientific linguistics. Several authors question the monoglot emphasis in the study of multilingualism (Y. Kachru, 1993, 1994; Sridhar 1994; Thumboo, 1994). Similarly, Pennycook (1994) argues that the very notion of "a language" as represented by the monolithic grammars created by most linguists is in fact "a very particular cultural and historical construct which reflects a specific "political psychology of nationalism, and an educational system devoted to standardizing the linguistic behavior of its pupils" (Harris as cited in Pennycook, 1994, p. 29).

${ }^{21}$ This view of language and identity as constitutive of one another is informing an increasing amount of work in critical linguistics and postmodern communication studies (e.g., Hecht, Collier \& Ribeau, 1993; Banks \& Banks, 1994; Jagtenberg, 1994). Leets et al. (1996) identify this emerging view as a "communication theory of identity."

${ }^{22}$ There is some controversy about whether, and to what extent, schema approaches should be distinguished from prototype and exemplar approaches (see Fiske \& Taylor, 1991, pp. 105-121). More recently authors developing a fuzzy logic approach have attempted to integrate the various lines of research (see Nosofsky's 1992 review of current debates and developments). Though there may be technical reasons to distinguish between these concepts for specific types of questions, there is no such need here. Therefore, following Fiske and Taylor's (1991) argument that the similarities between these knowledge structures outweigh their differences (p.117), I will use "schema" in its most general sense as a structured and selective internal representation within memory of some aspect of experience.

${ }^{23}$ Following a middle ground within the debate of linguistic determinism, schemata should be seen as predisposing--though not determining--an individual to experience the world in certain ways and not others. 
${ }^{24}$ Within traditional attribution theory, these become internalized in the form of a set of generalized bipolar attributes; however, keeping in mind Markus and Katayama's suggestion that this view may be culturally biased, I have chosen to be more general in description.

${ }^{25}$ The analogy of self knowledge as expert knowledge is common in the field. Fiske and Taylor's (1991) summary of the similarities in self memory recall and expert subject recall would suggest that the comparison is more than mere analogy.

${ }^{26}$ Winch (1958) has similarly argued that this conclusion follows directly from the philosophical foundations for a sociology of knowledge: "the concepts in terms of which we understand our own mental processes and behaviour have to be learned, and must, therefore, be socially established, just as much as the concepts in terms of which we come to understand the behaviour of other people" (p. 119).

${ }^{27}$ This may account for the phenomena of some isolated indigenous groups giving themselves names which mean something close to "the human beings," or "the people." For a discussion of several examples, see Appelbaum and Chanbliss, 1995, pp. $71-73$.

28 "This can sometimes have odd results. ... WASPS in the US are often designated as an ethnic group though few such persons consider themselves to be members of any such group" (Patterson, 1983, p. 32)

${ }^{29}$ This view of ethnic consciousness is consistent with the definition of culture given above. I repeat it here with special emphasis:

A culture from this perspective is that subset of possible or available meanings which by virtue of enculturation (informal or formal, implicit or explicit, unintended or intended) has so given shape to the psychological processes of individuals in a society that those meanings have become, for those individuals, indistinguishable from experience itself. 
${ }^{30}$ Though this comment by Morgan (1991) is actually in his criticism of the assumptions and methodologies of many studies of racial identity, it is also relevant here.

${ }^{31}$ B. Kachru $(1982 ; 1990)$ has discussed both the varied reasons for retention of colonial languages as well as the developmental stages leading to institutionalization..

${ }^{32}$ Since the nature of linguicultural monism specifies that any ethnic identity $\mathrm{X}$ is, and can only be, associated with language $X$, the potential conjunctions of 1 and $2 ; 1$ and $3 ; 3$ and 4 ; and 2 and 4 would necessarily be in structural conflict and therefore, unstable. These restrictions leave the two possibilities discussed above.

${ }^{33}$ Though on an individual level, the concept of integration is similar to that of constructive marginalism, it lacks the sense of social marked-ness that is present in the concept of marginalism. In societal situations of integration there may be no sense of marginality.

${ }^{34}$ In fact, the representations which are presented were produced in part using MDS. Originally, I had decided on these representations because they were conceptually consistent with the earlier discussion. Sometime after, I used a hypothetical set of identity labels to construct artificial sets of card sort data which represented logically extreme cases of sorting. The results of these artificial data sets were used to verify the presented spatial representations.

${ }^{35}$ This is not to say that a this identity construal cannot, in principle, be represented by a self schema conceptualization. More recent time series methodologies (see Demo, 1992) using a self schema conceptualization may be applicable to conditions characterized by folk multilingualism and integration. However a static time slice, or "snap shot" representation simply would not adequately represent the essential qualities of this state. 
${ }^{36}$ Depending on the type of research and the researcher, different terms have been used to refer to the things being compared. Though for some purposes there may be reason to distinguish between the terms "object," "mental object," and "stimulus object," I will use them synonymously. Sometimes the objects are not compared directly, but are compared through the use of a sign which refers to the object-e.g., use of labels on a card. I will refer to the signs that are used to represent these objects (words, descriptions, pictures, etc.,) as "stimuli," "labels," "stimulus labels."

${ }^{37}$ For detailed information on the relationship between distances and proximity measures, see Norušis (1992).

${ }^{38}$ An SPSS matrix language program was written specifically for this process. This program is available by contacting the author.

${ }^{39}$ This program is available by contacting the author. 


\section{References}

Abelson, R. P. (1981). The psychological status of the script concept. American Psychologist, $\underline{36}, 715-729$.

Aboud, F. \& Christian, J. (1979). Ethnic identity. In L. Eckensberger, W. Lonner, \& Y. Poortinga (Eds.), Cross-cultural contributions to psychology (pp.18-95).

Lisse: Swets \& Zeitlinger.

Agar, M. (1991). The biculture in bilingual. Language in society, 20, 167-181.

Allen, V. \& Wilder, D. (1975). Categorization, belief similarity and intergroup discrimination. Journal of Personality and Social Psychology, 32, 971-977.

Allen, V., Wilder, D., \& Atkinson, M. (1985). Multiple group membership and social identity. In T. Sarbin \& K. Scheibe. (Eds.), Studies in social identity (pp. 93115). New York: Praeger.

Allport, G. (1955). Becoming. New Haven: Yale University Press.

Appelbaum, R. \& Chanbliss, W. (1995). Sociology. NewYork: Harper \& Row.

Asmah, H. (1975). Essays on Malaysian linguistics. Kuala Lampur: Dewan Bahasa dan Pustaka.

Atkinson, D., Morten, C., \& Sue, G. (1983). Counseling American minorities. Dubuque, IA: Wm. C. Brown.

Bailey, K. (1975). Cluster Analysis. In D. Heise (Ed.), Sociological methodology (5928). San Fransisco, CA: Josey Bass.

Banaji, M. \& Prentice, D. (1994). The self in social contexts. Annual Review of Psychology, 45, 297-332.

Banks, A. \& Banks, S. (1994, July). Culture, identity, and "good theory: Implications for intercultural communication theory from Gypsy culture. Paper presented to the intercultural development division of the annual meeting of the international communication Association, Sydney, Australia.

Bannister, G. \& Fransella, F. (1971). Inquiring man: The theory of personal constructs. Harmonsworth: Penguin. 
Baxter, J. (1980). How should I speak English? American-ly, Japanese-ly, or International-ly. Jalt Journal, 2, 31-61.

Bennett, J. M. (1993). Cultural marginality: Identity issues in intercultural training. In R.M. Paige (Ed.), Education for the intercultural experience (pp. 109-136). Yarmouth, ME: Intercultural Press.

Berger, C. (1975). Proactive and retroactive attribution processes in interpersonal communications. Human Communication Research, 2 (1), 33-50.

Berger, C., \& Badac, J. (1982). Language and social knowledge: Uncertainty in interpersonal relations. London: Edward Arnold.

Berger, P., \& Luckmann, T. (1966). The social construction of reality: A treatise in the sociology of knowledge. Garden City, NY: Doubleday.

Berns, M. (1990). Contexts of competence: Social and cultural considerations in communicative language teaching. New York: Plenum.

Berry, J. (1986). The acculturation process and refugee behavior. In C. Williams \& J. Westermeyer (Eds.), Refugee mental health in resettlement countries (pp. 2952). New York: Hemisphere.

Berry, J., Poortinga, Y., Segall, M., \& Dasen, P. (1992). Cross-cultural psychology: Research and applications. New York: Cambridge.

Blummer, H. (1969). Symbolic interactionism. Englewood Cliffs: Prentice Hall.

Billig, M., \& Tajfel, H. (1973). Social categorization and intergroup behavior. European Journal of Social Psychology, $\underline{3}$, 27-52.

Blunk, P. (1994). Perceived communication during organizational change. Unpublished masters thesis, Portland State University, Portland, OR.

Bower, G., \& Gilligan, S. (1979). Remembering information related to one's self. Journal of Research in Personality, 13, 404-419.

Brewer M. (1991). The Social self: On being the same and different at the same time. Personality and Social Psychology Bulletin, 17 (5), 475-482. 
Brewer, M. \& Schneider, S. (1990). Social identity and social dilemmas: A double edged sword. In D. Abrams \& M. Hogg (Eds.). Social identity theory: Constructive and critical advances (pp. 120-149). London: HarvesterWheatsheaf.

Brislin, R. (1970). Back translation for cross-cultural research. Journal of Crosscultural Psychology, 1 (3), 185-216.

Brown, K. (1993). World Englishes in TESOL programs: An infussion model of curricular innovation. World Englishes, 12 (1), 59-74.

Carlson, E., \& Rosser-Hogan, R. (1993). Mental health status of Cambodian refugees ten years after leaving their homes. American Journal of Orthopsychiatry, $\underline{63}$ (2), 223-231.

Carstens, S. (1983). Pulai Hakka Chinese Malaysian: A laberynth of cultural identities. In L. Lim \& L. A. Gosling.(Eds.), The Chinese in southeast Asia: Identity, culture and politics (pp.79-96). Singapore: Maruzen Asia.

Carstens, S. (1986). Public and private definitions of cultural identity in a Chinese Malaysian settlement. In S. Carstens (Ed.), Cultural identity in northern peninsular Malaya (96-116). Athens, OH: Ohio Press.

Celano, M., \& Tyler, F. (1990). Behavioral acculturation among Vietnamese refugees in the United States. Journal of Social Psychology, 131 (3), 373-385.

Chan, K. B., \& Tong, C. K. (1992). Rethinking assimilation and ethnicity: The Chinese in Thailand. International Immigration Review, 27 (1), 140-168.

Chang, Y., \& Kapanga, M. (1992, April). The power and politics of English in a world context. Paper presented at the World Englishes Today Conference, Champaign, IL.

Cheshire, J. (Ed.). (1991). English around the world: Sociolinguistic perspectives. New York: Cambridge University Press.

Chun, K. (1985). Ethnicity and ethnic identity: Taming the untamed. In T. Sarbin \& K. Scheibe (Eds.), Studies in social identity (pp. 185-203). New York: Praeger.

Clyne, M. (1992). Pluricentric languages: Differing norms in differing languages. New York: Mouton de Gruyter. 
Combs, A., \& Snygg, P. (1959). Individual behavior. (2nd ed.). New York: Harper.

Conversi, D. (1990). Language or race?: The choice of core values in the development of Catalan and Basque nationalism. Ethnic and Racial Studies, 13, 51-70.

Cooper L. A. \& Shepard, R. N. (1984). Turning something over in the mind. Scientific American, 251, 106-115.

Cousins, S. (1989). Culture and self perception in Japan and the United States. Journal of Personality and Social Psychology, 56 (1), 60-67.

Crocker, J., Luhtanen, R., Blaine, B., \& Broadnax, S. (1994). Collective self-esteem and psychological well-being among white, black and Asian college students. Journal of Personality and Social Psychology Bulletin, 20, (5). 503-513.

Cronklin, N. \& Lourie, M. A. (1983). A host of tongues: Language communities in the United States. New York: Free Press.

Dasgupta, P. (1993). The otherness of English: India's auntie tongue syndrome. New Delhi: Sage.

Davison, M. (1983). Multidimensional scaling. New York: John Wiley \& Sons.

Davies, A. (1991). The native speaker in applied linguistics. Edinburgh: Edinburgh University Press.

Demo, D. (1992). The self concept over time: Research issues and directions. Annual Review of Psychology, 18, 303-326.

Denham, P. (1992). English in Vietnam. World Englishes, 11 (1), 61-69.

Deux, K. (1992). Personalizing identity and socializing self. In G. Breakwell (Ed.), The psychology of identity and the self concept. (pp. 9-33). London: Academic.

De Vos, G. (1982). Ethnic pluralism: conflict and accomodation. In G. De Vos \& L. Romanucci-Ross (Eds.), Ethnic identity: Cultural continuities and change (pp. 542). Chicago, IL: University of Chicago Press.

De Vos, G. (1985). Adaptive conflict and adjustive coping: Psycho-cultural approaches to ethnic identity. In T. Sarbin \& K. Scheibe (Eds.), Studies in social identity (pp. 204-228). New York: Praeger. 
Devereux, G. (1982). Ethnic identity: Its logical foundations and dysfunctions. In G. De Vos \& L. Romanucci-Ross (Eds.), Ethnic identity: Cultural continuities and change (pp. 43-71). Chicago,IL: University of Chicago Press.

De Vos, G., \& Romanucci-Ross, L. (9182). Vessel of meaning and emblem of contrast. In G. De Vos \& L. Romanucci-Ross (Eds.), Ethnic identity: Cultural continuities and change (pp. 363-389). Chicago, Il: University of Chicago Press.

Dillon, J. and Golstein, M. (1984). Multivariate analysis. New York: John Wiley \& Sons.

Doi, T. (1973). The anatomy of dependence. (J. Bester, Trans.). San Fransisco, CA: Kodansha International.

Eckennsberger, L., Lonner, W., Poortinga, Y. (Eds.). (1979). Cross-cultural contributions to psychology. Lisse: Swets and Zeitlinger.

Felix-Ortiz, M., Newcomb, D., \& Myers, H. (1994). A multidimensional measure of cultural identity for Latino and Latina adolescents. Hispanic Journal of Behavioral Sciences, 16 (2), 99-115.

Fernando, L. (1986). Cultures in conflict: Essays on literature \& the English language in south east Asia. Singapore: Graham Brash.

Finkelstein, B. (1991). Conflict in Japanese schools. In B. Finkelstein, A. Imamura \& J. Tobin (Eds.), Transcending stereotypes: Discovering Japanese culture and education (pp. 183-189). Yarmouth, ME: Intercultural Press.

Fiske, S. \& Dyer, L. (1985). Structure and development of social schemata: Evidence from positive and negative transfer effects. Journal of Personality and Social Psychology, 4ㅇ, 839-852.

Fiske, S., \& Taylor, S. (1991). Social cognition. New York: Mcgraw-Hill.

Gardner, R. \& Lambert, W. (1972). Attitudes and motivation in second language learning. Rowley, MA: Newbury House.

Ghee, K. (1990). The psychological importance of self definition and labeling: Black versus African American. The Journal of Black Psychology, 17, 175-193.

Gibson, M. (1988). Accomodation without assimilation: Sikh immigrants in an American high school. Ithica, NY: Cornell University Press. 
Giles, H., Bourhis, R., \& Taylor, D. (1977). Towards a theory of language in ethnic group relations. In $\mathrm{H}$. Giles (Ed.), Language, ethnicity, and intergroup relations (pp. 307-348). London: Academic Press.

Giles, H., Taylor, D., \& Bourhis, R. (1974). Dimensions of Welsh identity. European Journal of Social Psychology, 7, 165-174.

Giles, H., Taylor, D., Lambert W., \& Albert, G. (1975). Monograph. McGill University.

Goh, E. (1993). Standards of English as a world language. In A. Pakir (Ed.), The English language in Singapore: Standards and norms (pp. 13-18). Singapore: National University of Singapore.

Guiora, A. (1981). Language Personality and culture or the Whorfian-hypothesis revisited. In M. Hines \& W. Rutherford (Eds.), On TESOL 81 (pp. 169-177). Washington, DC: TESOL.

Guiora, A., Brannon, R., \& Dull, C. (1972). Empathy and second language learning. Language Learning, 22 (1), 111-130.

Hamnett, M., Porter, D., Signh, A., \& Kumar, K. (1984). Ethics politics, and international social science research: From critique to praxis. Honolulu, HI: University of Hawaii Press.

Hare, J., Anderson, R. Tatham, R. (1992). Multivariate data analysis. (3rd ed.). New York: Macmillan.

Hecht, M., Collier, M. \& Ribeau, S. (1993). African American communication. Newbury Park, CA: Sage.

Heider, F. (1958). The psychology of interpersonal relations. New York: Wiley.

Heller, M. (1987). The role of language in the formation of ethnic identity. In J. Phinney \& M. Rotheram (Eds.), Children's ethnic socialization: Pluralism and development (pp. 180-200). Newbury Park CA: Sage.

Hoelter, J. (1985). The structure of self conception: Conceptualization and measurement. Journal of Personality and Social Psychology, 49, 1392-1407.

Hoffman, C. (1991). An introduction to bilingualism. New York: Longman. 
Isajiw, W. W. (1980). Definitions of ethnicity. In R. Bienvenue \& J. Goldstein (Eds.), Ethnicity and ethnic relations. Toronto, Canada: Butterworths.

Jaeger, R. (1990). Statistics: A spectator sport (2nd ed.). Newbury Park, CA: Sage.

Jagtenberg, T. (1994). Lost and found: Dialectics of the self and identity. Paper presented to the annual conferences of the Australian and New Zealand Communication Association and the International Communication Association. The University of Technology, Sydney, Australia. July 11-15.

James, W. (1890). Principles of psychology. New York: Holt.

James, W. (1915). Psychology: A briefer course. New York: Holt.

Johnson, R., \& Wichern, D. (1982). Applied multivariate statistical analysis. Englewood Cliffs, NJ: Prentice-Hall.

Kachru, B. (1979b). Code-mixing and communicative strategy in India. In J. Richards (Ed.), New varieties of English: issues and approaches (pp. 18-33). Singapore: SEAMEO Regional Language Center.

Kachru B. (Ed.). (1982). The other tongue: English across cultures. Urbana, IL: University of Illinois Press.

Kachru, B. (1983). The Indianization of English: the English language in India. New York: Oxford University.

Kachru, B. (1987). The past and predjudice: Toward demythologizing the English cannon. In R. Steele \& T. Threadgold (Eds.), Language topics: Essays in honour of Michael Halliday (Vol. 12, pp. 45-256). Philadelphia, PA: John Benjamins.

Kachru, B. (1989). Teaching world Englishes. Indian Journal of Applied Linguistics, $\underline{15}(1), 85-94$.

Kachru, B. (1990). The alchemy of English: The spread, functions, and models of nonnative Englishes. Urbana, IL: University of Illinois Press.

Kachru, B. (1991, January). Liberation linguistics and the Quirk Concern. English Today, 17, 3-13.

Kachru, B. (1994). Teaching world Englishes without myths. In S. Kaur Gill (Ed.), Papers from the International English education conference: National and 
international challenges and responses. (pp. 10-23). Malaysia: Language Centre, Universiti Kabansan Malaysia.

Kachru, B. (Ed.). (1992). The other tongue: English across cultures (2nd ed.). Urbana: University of Illinois.

Kachru, Y. (1993). Reviews: Interlanguage and language acquisition research. World Englishes, 12 (2), 265-273.

Kachru, Y. (1994). Monolingual bias in SLA research. TESOL Quarterly, 28 (4), 795-799.

Kahane, H. (1982). American English: From colonial substandard to a prestige language. In B. Kachru (Ed.), The other tongue: English across cultures (2nd ed.) Urbana, IL: University of Illinois.

Kandiah, T. \& Kwan-Terry, J. (1994). English and language planning: A southeast Asian contribution. Singapore: Times Academic Press

Keefe, S., Reck, G., \& Reck, U. (1987). Measuring ethnicity and it's political consequences in a southern Appalachian high school. Negotiating ethnicity: The impact of anthropological theory and practice. (Bulletin No. 8, pp. 27-35). National Association for the Practice of Anthropology.

Keef, S.E., \& Padilla, A. (1987). Chicano ethnicity. Albuquerque: University of New Mexico Press.

Kelly, G. (1955). The psychology of personal constructs. New York: Norton.

Kernberg, O. (1977). Borderline conditions in pathological narcissism. International University Press.

Khan, V., Baric, R., Graumann, C., Heckmann, F., Mardin, S., Morin, F., Patterson, S., Pettigrew, T., Pradellesde Latour, M., Schiffauer, W., Wallman, S., \& Yalcin, L. (1983). Formation of consciousness, group report. In C. Fried (Ed.), Minorities:Community and identity (pp. 95-107). New York: Springer-Verlag.

Khubchandani, L. M. (1983). Plural languages, plural cultures: Communication, identity and socio-political change in contemporary India. Honolulu, $\mathrm{HI}$ : University of Hawaii. 
Khun, M., \& McPartland, T. (1954). An empirical investigation of self attitudes. American Sociological Review, 19, 68-76.

Kernberg, O. (1977). Borderline conditions on pathological narcissism. International University Press.

Kihlstrom, J., \& Cantor, N. (1984). Mental representations of the self. In L. Berkowitz (Ed.), Advances in Experimental Social Psychology (Vol. 17, pp. 248). New York: Academic Press.

Kihlstrom, J., Cantor, N., Albright, J., Chew, B., Klein, S., \& Niedenthal, P. (1988). Information processing and the study of the self. In L. Berkowitz (Ed.), Advances in Experimental Social Psychology (Vol. 21, pp. 145-178). New York: Academic Press.

Kono, N. (1995). A cross-cultural study of classroom culture in a foreign language setting. The ORTESOL Journal, 16, 69-76.

Kochman, T. (1981). Black and white styles in conflict. Chicago: University of Chicago Press.

Kramsch, C. (1993). Context and culture in language teaching. Oxford: Oxford University.

Krewer, B., \& Ekensberger, L. (1989). Cultural Identity and Political Events. In D. M. Keats, D. Munro \& L. Mann (Eds.), Hetrogeneity in cross-cultural psychology (pp. 216-231). Amsterdam: Swets and Zeitlinger.

Kruskal, J., \& Wish, M. (1978). Multidimensional scaling. Beverly Hills CA: Sage.

Kwan -Terry, J. (1991). Ulysses circling the merlion: The invention of identity in Singapore poetry in English and Chinese. In E. Thumboo (Ed.), Percieving other worlds (pp. 115-138). Singapore: Unipress.

Lalonde, R., Taylor, D., \& Moghaddam, F. (1992). The process of social identification for visible immigrant women in a multicultural context. Journal of Cross-Cultural Psychology, 23 (1), 25-39.

Lambert, W. (1969). Psychological Aspects of Motivation in Language Learning, Florida FL Language Reporter. Spring/Summer, 96-97. 
Lambert, W. (1992). Challenging established views on social issues: The power and limitations of research. American Psychologist, 47, 543-542.

Laroche, M., Kim, C., Hui, M., Joy, A. (1996). An empirical study of multidimensional ethnic change. Journal of Cross-cultural Psychology, 27 (1), 114-131.

Leets, L., Giles, H. \& Clement, R. (1996). Explicating ethnicity in theory and communication research. Multilingua, $\underline{15}$, 116-147.

Llamzon, T. (1983). A new approach to the teaching of English in the Philippines. In J. Richards (Ed.), New varieties of English: Issues and approaches (pp. 131-140). Singapore: SEAMEO Regional Language Center.

Littlejohn, S. (1989). Theories of human communication (3rd. ed.). Belmont, CA: Wadsworth Publishing.

Lopez, D., \& Espiritu, Y. (1990). Panethnicity in the United states: A Theoretical Framework. Ethnic and Racial Studies, 13, 199-224.

Lowenberg, P. (1984). Non-native varieties varieties of English: Nativization, norms, and implications. Studies in Second Language Acquisition, $\underline{8}, 1-18$.

Lowenberg, P. (Ed.). (1988). Language spread and language policy: Issues, implications, and case studies. Washington: Georgetown University.

Lowenberg, P. (1993, March). Myths and realities in testing world Englishes. Paper presented at the First Annual Conference of the International Association of World Englishes, Urbanna, IL.

Luhtanen, R. \& Crocker, J. (1991). Self esteem and intergroup comparisons: Toward a theory of collective self esteem. In J. Suls \& T. Wills (Eds.), Social comparison (pp. 211-34). Hillsdale, NJ: Erlbaum.

Luhtanen , R., \& Crocker, J. (1992). A collective self esteem scale: Self evaluation of one's social identity. Personal and Social Psychology Bulletin, 18, 302-318.

Mahapatra, B. (1983). Ethnicity, Identity, and Language. Indian Linguistics, $44,61-$ 66.

Marcia, J. (1980). Identity in Adolescence. In J. Adelson (Ed.), Handbook of adolescent psychology (pp. 159-187). New York: Wiley. 
Markus, H., \& Kitayama, S. (1991). Culture and the self: implications for Cognition, Emotion and Motivation. Psychological Review, 98, 224-253.

Markus, H., \& Kitayama, S. (1992). Cultural variation in the self concept. In G. Goethals \& J. Strauss (Eds.), Multidisciplinary perspectives on the self (pp. 2747). New York: Springer-Verlag.

Markus, H., \& Kitayama, S. (1994 in press). A collective fear of the collective: implications for selves and theories of selves. Personality \& Social Psychology Bulletin.

Markus, H., Smith, J., \& Moreland, R. (1985). Role of self concept in the perception of others. Journal of Personality and Social Psychology, 49, 1494-1512.

Markus, H., \& Nurius, P. (1986). Possible selves. American Psychologist, 41, 954969.

Markus, H., \& Sentis, K. (1982). The Self in Social Information Processing. In J Suls (Ed.), Psychological perspectives on the self (Vol. 1, pp. 41-69). Hillsdale New Jersey: Lawrence Erlbaum \& Associates.

Markus, H., \& Wurf, E. (1987). The dynamic self concept: A social psychological perspective. Annual Review of Psychology, $\underline{38}$, 299-337.

Martin, L., \& Clark, L. (1990). Social cognition: Exploring the mental processes involved in human social interaction. In M. Eysenck (Ed.), Cognitive Psychology: An International Review (Vol. 1, pp. 266-310). Sussex England: Wiley.

McGuire, W., \& McGuire, C. (1982). Significant others in self space: Sex differences and developmental trends in the social self. In J. Suls (Ed.), Psychological Perspectives on the Self (Vol. 1, pp. 71-95). Hillsdale, NJ: Lawrence Erlbaum \& Associates.

Masuda, M., Matsumoto, G. H., \& Meredith, G. M. (1970). Ethnic identity in three generations of Japanese Americans. Journal of Social Psychology, 16, 136-146.

Mead, G. (1934). Mind, self and society. Chicago: University of Chicago.

Moghaddam, F. M., \& Taylor, D. (1987). The meaning of multiculturalism for visible minority immigrant women. Canadian Journal of Behavioural Science, 19 , 121136. 
Mohanan, K. (1992). Describing the phonology of non-native varieties of a language. World Englishes, 11 (2/3), 111-128.

Montaner, C. (1990). Talk English--You are in the United States. In J. Crawford (Ed.), Language Loyalties: A source book on the English only controversy (pp. 163-165). Chicago: University of Chicago Press.

Monzel, K. (1987). A Geographical Perspective on the Acculturation and Satisfaction of an Indo-Chinese Refugee Community. Unpublished masters thesis, University of Colorado, Boulder, CO.

Morgan, H. (1991). Doll studies revisited: Is the message in the method? The Western Journal of Black Studies, 15 (4), 248-253.

Nishida, H. (1989). Jitsurei de minu nichibeicommunication gaps. [Comunication gaps between japanese and Americans: In case studies]. Japan: Taishuukan shoten.

Norušis, M. (1992). SPSS for windows: Professional statistics, release 5.0. Chicago, IL: SPSS.

Northover, M. (1988). Bilinguals or Dual Linguistic Identities. In J. Berry \& R. Annis (Eds.), Ethnic psychology: Research and practice with immigrants, refugees, native peoples, ethnic groups, and sojourners (pp. 207-216). Berwyn, PA: Swets North America.

Nosofsky, R. (1992). Similarity scaling and cognitive models. Annual Review of Psychology, 43, 25-53.

Noss, R. (Ed.). (1991). Varieties of English in southeast Asia. Singapore: Singapore University Press.

Paikeday, T. (1985). The native speaker is dead. New York: Paikeday Publishing.

Pakir, A. (1993). The English language in Singapore: Standards and norms. Singapore: National University of Singapore.

Pandharipande, R. (1992). Defining politeness in Indian English. World Englishes, 11 (2/3), 241-250.

Park, R.E. (1950). Race and culture. Glencoe, Il: Free press. 
Patterson, O. (1983). The Nature, Causes, and Implications of Ethnic Identification.

In C. Fried (Ed.), Minorities: Comunity and identity (pp. 25-50). New York: Springer-Verlag.

Pennycook, A. (1994). The cultural politics of English as an international language. New York: Longman.

Peterson, J. (1994). Conceptualizing ethnic identity. Paper presented at the First Annual Meeting of the International Association for World Englishes. Urbana , II.

Phillipson, R. (1992). Linguistic imperialism.Oxford: Oxford University.

Phinney, J. (1989). Stages of ethnic identity in minority group adolescents. Journal of Early Adolescence, 9, 34-49.

Phinney, J. (1990). Ethnic Identity in Adolescents and Adults: Review of Research Psychological Bulletin, 108 (3), 499-514.

Pierson, H., Fu, G., \& Lee, S. (1980). An Analysis of the relationship between language attitudes and English attainment of secondary students in Hong Kong. (Research Report). Hong Kong: Social Research Center.

Pradell de Latour, M. (1983). Identity as a Complex Network. In C. Fried (Ed.), Minorities: Community and identity (pp. 79-92). New York: Springer-Verlag.

Prator, C. (1968). The British heresy in TESL. In J. Fishman, C. Ferguson \& J. Das Gupta (Eds.), Language problems in developing nations (pp. 145-166). New York: Wiley \& Sons.

Preston, D. (1989). Sociolinguistics and second language acquisition. New York: Basil Blackwell.

Pride, J. (1983). Communicative Needs in the Use and Learning of English. In J. Richards (Ed.), New varieties of English: Issues and approaches (pp. 86-79). Singapore: SEAMEO Regional English Language Center.

Quirk, R. (1987). The question of standards in the international use of English. Language spread and language policy: Issues, implications, and case studies. (pp. 331-341). Georgetown university roundtable on language and linguistics. 
Ree, J. (1980). English for Asians as a second language: Problems and strategies. In J. Koo and R. St. Clair. (Eds.), Bilingual education for Asian Americans: Problems and strategies (pp. 15-23). Hiroshima, Japan: Bunka Hyoron.

Reminik, R. (1983). Theory of ethnicity: An anthropologist's perspective. New York: University Press of America.

Richmond, A. (1988). Immigration and ethnic conflict. London: Academic Press.

Rin, H. (1982). The Synthesizing Mind in Chinese Ethno-Cultural Adjustment. In G. DeVos \& L. Ramanucci-Ross (Eds.), Ethnic identity: Communities and change (pp. 137-155). Chicago IL: University of Chicago Press.

Robinson, G. (1991). Second culture acquisition. In J. Alatis. (Ed.), Georgetwon University Roundtable on languages and linguistics. Linguistics and language pedogogy: State of the art (pp. 114-21). Washington, DC: Georgetwon University.

Rodby, J. (1992). A polyphony of voices: The dialectics of linguistic diversity and unity in the twentieth-century United States. In T. Machan \& S. Chas (Eds.), English in its social contexts: Essays in historical sociolinguistics (pp. 73-97). New York: Oxford.

Rosenburg, S., Nelson, C., \& Vivekananthan, P. (1968). A multidimensional approach to the structure of personality impressions. Journal of Personality and Social Psychology, $\underline{9}$, 283-294.

Rosenberg, S., \& Sedlak, A. (1972). Structural representations of implicit personality theory. In L. Berkowitz. (Ed.), Advances in Experimental Social Psychology (Vol. 6, pp. 235-297).

Sarbin, T. (1962). A preface to a psychological analysis of the self. Psychological Review, 59, 11-22.

Sayegh, L., \& Lasry, J. (1994). Immigrants' adaption in Canada: Assimilation, acculturation, and orthogonal cultural identification. Canadian Psychology, 34 (1), 99-109.

Schuman, J. (1976). Social distance as a factor in second language learning. Language Learning, 26, 135-143. 
Schuman, J. (1978). The Acculturation Model for Second Language Acquisition. In R. Gingras (Ed.), Second language acquisition and foreign language teaching. Washington, DC: Center for Applied Linguistics.

Shen, F. (1989). The classroom and the wider culture: Identity as a key to English composition. College Composition and Communication, 40, 459-466.

Shepard, R. (1966). Metric structures in ordinal data. Journal of Mathematical Psychology, $\underline{3}, 287-315$.

Shimanoff, S. (1980). Communication rules: Theory and research. Beverly Hills, CA: Sage.

Shweder, R., \& Sullivan, M. (1993). Cultural psychology: who needs it? Annual Review of Psychology, 44, 497-523.

Shweder, R., \& Bourne, E. (1984). Does the concept of person vary cross-culturally? In R. Shweder \& R. Levine (Eds.), Culture theory: Essays on mind, self, and emotion (pp.158-199). New York: Cambridge University Press.

Shweder, R., \& LeVine, R. (Eds.). (1984). Culture theory: Essays on mind, self, and emotion. New York: Cambridge.

Singelis, T. (1994). The measurement of independent and interdependent self construals. Personality and Social Psychology Bulletin, 20, 580-591.

Smith, L. (1991). Standards in world Englishes. In M. Tickoo (Ed.), Languages \& standards: Issues, attitudes, case studies (pp. 33-39). Singapore: RELC.

Smith, L. (1981). English for cross-cultural communication. Honalulu, HI: East-West Center.

Smolicz, J. (1980). Language as a Core Value of Culture. Regional English Language Journal, 11 (1), 1-14.

Smolicz, J. (1981a). Core values and cultural identity. Ethnic and Racial Studies, $\underline{4}$ (1), 75-89.

Smolicz, J. (1981b). Meaning and Values in Cross cultural Contacts. Revue International de Sociology, 17 (2-3), (pp. 55-73). 
Smolicz, J. (1985). Italian Language and Culture in South Australia: A memoir Approach. In C. Bettoni (Ed.), Italian abroad: Studies on language contact in English speaking countries (pp. 37-51). Sydney: Fredrick May Foundation for Italian Studies.

Smolicz, J. (1986). National Policy on Languages: A Community Language Perspective. Australian Journal of Education, 30 (1), 45-65.

Smolicz, J. (1992). The monolingual myopia and minority language rights: Australia's language policies from an international perspective. The Indian Journal of Social Science, $\underline{5}$ (2), 207-226.

Smolicz, J., \& Secombe, M. (1985b). Community Languages, Core Vaolues and Cultural maintenance: The Australian experience with special reference to Greek, Latvian, and Polish Groups. In M. Clyne (Ed.), Australia: Meeting place of languages (pp. 11-38). Australia: Pacific Linguistics.

Sperber D. \& Wilson, D. (1988). Relevance: Communication and cognition. Cambridge MA: Harvard.

Sridhar, S. N. (1994). A reality check for SLA theories. TESOL Quarterly, 28 (4), 800-803.

Stigler, J., Shweder, R., \& Herdt, G. (Eds.). (1990). Cultural psychology: Essays on comparative human development. New York: Cambridge.

Tajfel, H. (1970). Experiments in inter-group discrimination. Scientific American, $\underline{223}, 96-102$.

Tajfel, H., \& Billig, M. (1974). Familiarity and categorization in intergroup behavior. Journal of Experimental Social Psychology, 10, 159-170.

Tajfel, H., Billig, M., Bundy, R., \& Flament, C. (1971). Social categorization and intergroup behavior. European Journal of Social Psychology, 1, 149-178.

Taylor, D. (1975). Ethnic identity: Some cross-cultural comparisons. In J. W. Berry \& W. J. Lonner (Eds.), Applied cross-cultural psychology (pp. 168-173). Amsterdam: Swets and Zeitlinger.

Taylor, D., Bassili, J., \& Aboud, F. (1972). Ethnic identification in Canada: a crosscultural investigation. Canadian Journal of Behavioural Science, 4, 13-20. 
Taylor, D., Bassili, J., \& Aboud, F. (1973). Dimensions of ethnic identity: An example from Quebec. Journal of Social Psychology, 오, 185-192.

Thumboo, E. (1994). Language into Languages: Some conjugations of choice in Singapore. In T. Kandiah \& J. Kwan-Terry. (Eds.). English and language planning: A southeast Asian contribution Singapore: Times Academic Press.

Tickoo, M. (Ed.). (1991). Languages \& standards: Issues, attitudes, case studies. Singapore: SEAMEO Regional English Lanaguage Center.

Tollefson, J. (1989). Alien winds: The re-education of America's indochinese refugees. New York: Preager.

Tollefson, J. (1991). Planning language, Planning inequality: language policy in the community. New York: Longman.

Tran, T. V. (1987). Ethnic community supports and psychological well being of Vietnamese refugees. International Migration Review, 21, 833-844.

Tran, T. V. (1990). Language acculturation among older Vietnamese refugee adults. The Gerontologist, 30, 94-99.

Turner, J. (1987). Rediscovering the social group: A self categorization theory. New York: Basil Blackwell.

Vaughan, G. (1987). A Social Psychological Model of Ethnic Identity Development. In J. Phinney \& M. Rotheram (Eds.), Children's ethnic socialization: Pluralism and development (pp. 73-91). Newbury Park, CA: Sage.

Verkuyten, M. (1992). Ethnic group preferences and the evaluation of ethnic identity among adolescents in the Netherlands. The Journal of Social Psychology, 132, 741-750.

Wallman, S. (1983). Identity Options: Formation of consciousness, group report. In C. Fried (Ed.), Minorities:Community and identity (pp. 69-78). New York: Springer-Verlag.

Weinreich, P. (1980). Manual for identity exploration using personal constructs. London: Social Science Research Council.

Weinreich, P. (1988). The operationalization of ethnic identity. In J.W. Berry \& R. Annis (Eds.), Ethnic psychology: Research and practice with immigrants, 
refugees, native peoples, ethnic groups, and sojourners (pp. 149-168). Berwyn, PA: Swets North America.

Wierzbicka, A. (1991). Japanese key words and core cultural values. Language in Society, 20, 333-385.

Winch, P. (1958). The idea of a social science and its relation to philosophy. New York: Humanities Press.

Wolfram, W., Christian, D., \& Hatfield, D. (1986). The English of adolescent and young adult Vietnamese refugees in the United States. World Englishes, $\underline{5}, 47-$ 61.

Wong-Rieger, D. (1982). Mismatches in self identity and the adaption of Asian students to living in North America. In R. Rath, H.S. Asthana, D. Sinha \& J. P. B. Sinha (Eds.), Diversity and unity in cross-cultural psychology (pp. 129-147). Lisse: Swets and Zeitlinger.

Wong-Rieger, D. \& Quintana, D. (1987). Comparative acculturation of Southeast Asian and Hispanic immigrants and sojourners. Journal of Cross-cultural psychology, 18 (3), 345-62.

Woon, Y-F. (1985). Ethnic identity and ethnic boundaries: The Sino-Vietnamese in Victoria, British Columbia. Review of Canadian sociology and anthropology, 22 (4), 534-558.

Yao, E., (1983). Ethnic awareness of Chinese-American teenagers. Urban Education, 18 (1), 71-81. 
APPENDIX A

INFORMED CONSENT FORMS 


\section{Informed Consent [TST Stage]}

I (please print your name) agree to

take part in this research project on how different people define their identities. I understand that this study involves up to three different activities. These activities include:

1) Describing myself -- 15 - 30 minutes.

2) Deciding which descriptions are most similar -- 10 - 15 minutes.

3) Discussing the reasons for my ideas -- 15 -- 60 minutes.

I understand that each activity will happen during separate appointments, that I may choose to do only one of these activities, and that accepting to volunteer for one activity does not commit me to doing more than one.

I understand that I can refuse to be contacted again. I also understand that beginning an activity does not commit me to finishing. I can stop my involvement at any time before or during the activities. I also understand that if I do not feel comfortable with my participation after I am finished, that I may refuse to hand in my materials.

If you do NOT want to be contacted again, initial the following space:

Jay Peterson has told me that the purpose of this study is to examine how people think about themselves. He has offered to answer any questions I have about the study and what I am expected to do.

He has also promised that all information which I give will be kept confidential to the extent permitted by law, that the names of all the people in this study will remain confidential, and that after this study is finished all individual names will be destroyed.

I understand that I may not receive direct benefit from taking part in this study. However, the study may help to increase knowledge that might aid others in the future.

I understand that I don't have to take part in this study and that making this choice will not affect my official relationship with Portland State University, any other school, or any other official agency in any way.

I understand that there are no expected risks to me, but if at a later date I either experience problems as a result of my participation, or have further questions concerning my participation, I may contact Jay Peterson at 774-3683.

Date: Signature:

If you have concerns or questions about this study, please contact the Chair of the Human Subjects Research Review Committee, Office of Research and Sponsored Projects, 105 Neuberger Hall, Portland State University, 503/725-3417 


\section{Informed Consent [Card sort stage]}

I

take part in this research project on how different people define their identities.

(please print your name) agree to

I understand that this study involves the following:

1) Deciding which descriptions are most similar -- 20-40 Minutes

2) Discussing the reasons for my ideas.

I understand that I may be contacted at a later date to informally discuss my ideas further. I also understand that I can refuse to be contacted or refuse to discuss my ideas at any time.

If you do NOT want to be contacted again, initial the following space:

Jay Peterson has told me that the purpose of this study is to examine how people think about themselves.

He has offered to answer any questions I have about the study and what I am expected to do.

He has also promised that all information which I give will be kept confidential to the extent permitted by law, that the names of all the people in this study will remain confidential, and that after this study is finished all individual names will be destroyed.

I understand that I may not receive direct benefit from taking part in this study.

However, the study may help to increase knowledge that might aid others in the future.

I understand that I don't have to take part in this study and that making this choice will not affect my official relationship with Portland State University, any other school, or any other official agency in any way.

I understand that there are no expected risks to me, but if at a later date I either experience problems as a result of my participation, or have further questions concerning my participation, I may contact Jay Peterson at 774-3683.

Date: Signature:

If you have concerns or questions about this study, please contact the Chair of the Human Subjects Research Review Committee, Office of Research and Sponsored Projects, 105 Neuberger Hall, Portland State University, 503/725-3417 
APPENDIX B

INSTRUCTION GUIDES, INSTRUMENTS, AND DEMOGRAPHICS FORMS FOR TST STAGE 
Directions for TST stage

\section{Scripts for First Stage of Data Collection: Generating Self Descriptions.}

\section{General Directions:}

The purpose of this exercise is to brainstorm descriptions about yourself that you think are important for understanding who you are.

I will ask you to write your ideas in three different lists.

We will work on one list at a time. Use the paper that I will provide you.

All answers will be kept confidential. Only a small number of researchers will see these lists. The only reason I need your names is to contact you again. I will be the only one to see your names.

This is not a race. The order that you write down your ideas doesn't matter. There are no right or wrong answers.

Each list is individual to you. I am interested only in what you think about yourself.

Do not look at anyone else's list. Do not talk with anyone else while you think or write. Try to think until you either fill each list completely, or I tell you to stop.

\section{Directions for First List:}

For the first list use the paper labeled "List 1"

Imagine that you are going to have a pen pal. The pen pal has not been chosen yet. you don't know who it will be, but you are beginning to think about the first letter that you will write. In your first letter you want to describe who you are and you have decided that before you write the letter, you want to brainstorm a list of descriptions about yourself. Some of these descriptions you would probably use in the first letter, and some of them you would probably not use. The list is only for your own thinking.

What things about you do you think are most important for understanding who you are?

Answer using a single word, a phrase, or even a sentence. You may write in any language you want. This is not a writing test. Write your answer however you want.

The order of your ideas doesn't matter. The most important thing is to think of as many answers as possible to the question: "who am I?"

If you have any questions please raise your hand and I'll come to you. 
[ Between two and five minutes of writing monitor the group. When they begin to have difficulty read the following: ]

By now it may be getting difficult to think of more descriptions. I will try to help you think of more. First, draw a line across your paper under your last description -- show on board. Now think about how your answers might be different if different people asked you who you are? As you think of new descriptions, add them to your list. Imagine that many different people, and kinds of people, ask you this question. Now, try to finish your list.

\section{Directions for Second List:}

For the second list use the paper labeled "List 2"

Imagine that you are continuing to brainstorm a list for your first letter to your new pen pal. Remember, the pen pal has not been chosen yet and you don't know who it will be.

For this second list, I want you to think about the most important things for understanding who you are ethnically and culturally. You may be as general or specific as you want, but concentrate on who you are ethnically or culturally.

You may answer using a single word, a phrase, or even a sentence.

This is not a writing or grammar test. Write your answer however you want. You may write in any language you want.

If you think of a description which was in your first list write it down in this list agian.

Remember the order of your descriptions doesn't matter. There are no right or wrong answers. I am interested only in what you think about yourself. The most important thing is to think of as many possible answers to the question: "who am I ethnically and culturally?"

If you have any questions please raise your hand and I'll come to you.

[ Between two and five minutes of writing monitor the group. When they begin to have difficulty read the following: ]

By now it may be getting difficult to think of more descriptions. I will try to help you think of some more. First, please draw a line across your paper under your last description. Now think about how your answers might be different if different people asked you who you are ethnically and culturally. Imagine that as many different people, and kinds of people, ask you who you are ethnically and culturally. As you think of new descriptions, add them to your list. Now, try to finish your list. 
Directions for Third List:

For the last list use the paper labeled "List 3"

Again, imagine that you are continuing to brainstorm a list for your first letter to your new pen pal. Remember, the pen pal has not been chosen yet and you don't know who it will be. I want you to think about the languages that are important to understanding who you are.

This does not necessarily mean only those languages that you can speak. Think about the languages that are in your life in any way at all. If you think that in any way a language could be important for describing who you are, then write it down.

You may be as general or specific as you want. You may answer using a single word, a phrase, or even a sentence. You may write in any language you want.

If you think of a description which was in your first list write it down in this list again.

Remember, there are no right or wrong answers. I am interested in what you think about yourself. Write in any language you want. Write your answers however you want. If you have any questions please raise your hand and I'll come to you.

[ Between two and five minutes of writing monitor the group. When they begin to have difficulty read the following:]

By now it may be getting difficult to think of more answers. I will try to help you think of more. Please draw a line across your paper under your last answer. Now think about how your answers would be different if different people asked you what languages are important for describing who you are life. Imagine that many different people, and kinds of people ask you this question. Now, try to finish your list. 
[TST instrument \#1]

You have a new pen pal and are planning to write your first letter. You want to describe who you are. What things do you think are most important for understanding who you are? Think of as many answers as possible.

1)

2)

3)

4)

5)

6)

7)

8)

9)

10)

11)

12)

13)

14)

15)

16)

17)

18)

19)

20) 
[TST instrument \# 2]

What things do you think are most important for understanding who you are ethnically and culturally? Think of as many answers as possible. If you think of something already on the previous list, repeat it here.
1)

2)

3)

4)

5)

6)

7)

8)

9)

10)

11)

12)

13)

14)

15)

16)

17)

18)

19)

20) 
[TST instrument \#3]

What languages are important for describing who you are? Think of as many answers as possible. If you think of something already on the previous lists repeat it here.

1)

2)

3)

4)

5)

6)

7)

8)

9)

10)

11)

12)

13)

14)

15)

16)

17)

18)

19)

20) 


\section{Personal information}

First Name Family Name:

Do you have names in any other language or languages? (circle one) Yes No If so what are they:

Name

Language

1)

2)

3)

Circle one: Male Female Age:

Are you married? Yes No

Where were you born? Country:

City:

Do you work? If so, what do you do?

Year in school:

Major:

What job or career do you wish to have after you finish school?

How many years have you lived in the United States?

Where:

1)

2)

3) 
Years of education in any other country:

Years of education in the United States:

Did you take English as a second language classes in high school? (circle one) Yes No If yes, how long? (months / years)

Did you take ESL ENNR or ENNL classes at a community college or university? Yes No If yes, how long? (months / years)

Did you spend time in a transition camp? (circle one) Yes No

Months of school in transition camps:

Are you taking any language classes now? which ones?

1)

2)

3)

What languages do you know?

1)

2)

3)

What languages does your mother know?

1)

2)

What languages does your father know?

1)

2)
4)

5)

6)

3)

4)

3)

4) 
How many brothers do you have?

How many sisters do you have?

Did anyone else live in your home with you for more than a year? (grandparents aunts uncles, family friends, etc.,) If so, who?

1)

2)

3)

Circle one: I want to stay in the United States.

Someday I want to move back to the country where I was born.

Are you a member of any ethnic or cultural organizations? (circle one) Yes No If so, which ones?

1)

2)

3)

Thank you very much for your help. Would you please give me your phone number where I can contact you in case I have other questions.

Phone:

If you would like a copy of the results please include your address and I will send you a copy.

Address: 


\section{APPENDIX C}

\section{DATA COLLECTION AND DEMOGRAPHICS}

FORMS FOR CARD SORT STAGE 
Direction guide for card sort stage

\section{[After signing consent form]}

THANK YOU for volunteering to participate.

Do each part in order. When you finish a section, check with me before moving on.

\section{PART 1.}

You have been given 24 descriptions. The descriptions relate to different kinds of people, different characteristics of people, and different beliefs of people. Sort the descriptions into piles, placing those descriptions that you feel are similar into the same pile. In addition, each pile should be thought of a type of person.

There are no limits on the number of descriptions in each pile. A pile can have only one card, or it can contain all of the cards.

There is no limit on the number of piles. Use as many piles as you wish. If you want, you can also use a miscellaneous pile.

Many of the descriptions might fit in more than one pile. If you want to put a description in more than one pile use another copy from the plastic holder.

[after about 3-5 minutes]

You may have wanted to place one card in more than one pile. Right now that is not possible. You must choose the best pile for each card.

[record results when finished]

\section{PART 2.}

You have been given a plastic sheet containing copies of each of the descriptions that you just sorted. If you wish, you will now be able to place extra copies in as many piles as you wish.

You may also add, eliminate, or rearrange the groups in any way that you wish.

[after finished, give the subject a pile of pink blank cards]

Please think of a name for each pile of descriptions. You may use a description from the pile, or a name of your own. 
PART 3.

Turn to page the sheet labeled part 3.

Read the first sentence. Choose five descriptions from your piles which best describe how you are when you are with your parents. The descriptions which you choose do not have to come from the same pile.

Write the numbers of the five descriptions on the lines I have provided.

When you have finished with the first sentence, do the same with each of the other sentences.

\section{PART 4.}

You have been handed 16 additional descriptions. Add them into the piles which you created during PART 1.

If you feel that the description "fits" into a pile, then add it to the pile. You can put each description in as many piles as you like

However, DO NOT CHANGE THE ORIGINAL NUMBER OF PILES. DO NOT CHANGE THE POSITIONS OF THE ORIGINAL DESCRIPTIONS

[When finished instruct the subject to fill in the Demographics information]

[Place a rubber band around each pile of cards along with the name for the pile and place it into the envelope along with the demographics information] 
Data sheets for card sort stage

\section{Part 1.}
A.
B.
C.
D.
E.
F.
G.
$\mathrm{H}$.
I.

Part 3.

1. How I am with my parents

2. How I am with my brothers and sisters

3. How I am with my friends

4. How: I am at school

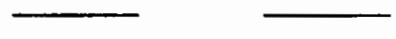

5. How I am at work 


\section{Final data [all 45 labels]}

Pile name:

A.

A.

Pile name:

B.

Pile name:

c.

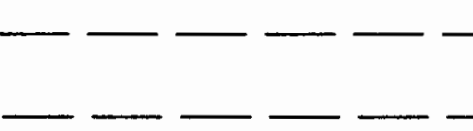

Pile name:

E.

Pile name:

F.

Pile name:

G.

Pile name:

H. 
Pile name:

I.

Pile name:

J.

Pile name:

K.

Pile name:

L.

Pile name:

M.

Pile name:

N.

le name

0.

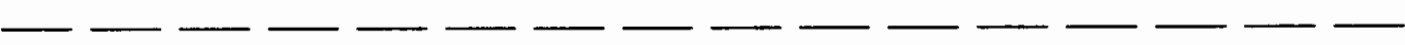




\section{Personal Information}

1. Sex: ( ) Male ( ) Female

2. Age:

3. Country where you were born:
( ) Vietnam
( ) China
( ) USA
( )
Other:

4. Years in the US:

5. In which of the following languages do you have names:
( ) Vietnamese
( ) Chinese
( ) English
( ) Other :

\section{School in Vietnam}
( ) None
( ) Grades $1-5$
() Grades 6 - 8
( ) Grades $9-12$
( ) College

7. School in the US:

( ) None

( ) Grades $1-5$

( ) Grades 6 - 8

( ) Grades $9-12$

( ) College

8. What is your educational level:
( ) High school graduate
( ) In college now
( ) College graduate

9. Year in college
( ) Freshman
( ) Sophomore
( ) Junior
( ) Senior

10. Major:

\section{Intended career:}

12. What is your job now?

13. Did you take ESL in high school
( ) Yes
( ) No

14. Did you take ESL / ENNR / ENNL in college?
( ) Yes
( ) No

15. Do you belong to any of the following organizations?
( ) None
( ) Vietnamese student organization
( ) Vietnamese community organization
( ) Vietnamese Church
( ) Other:

16. Which languages does your mother know:
( ) Vietnamese
( ) English
( ) Chinese
( ) French
( )
Other.

17. Which languages does your father know:
( ) Vietnamese
( ) English
( ) Chinese
( ) French
( )
Other.

18. Which languages do you know:
( ) Vietnamese
( ) English
( ) Chinese
( ) French
( )
Other. 


\section{APPENDIX D}

Similarity Matrices 


\begin{tabular}{|c|c|c|c|c|c|c|c|c|c|c|c|c|c|c|c|c|c|c|c|c|c|c|}
\hline & 1 & 2 & 3 & 4 & 5 & 6 & 7 & $\boldsymbol{8}$ & 9 & 10 & 11 & 12 & 13 & 14 & 15 & 16 & 17 & 18 & 19 & 20 & 21 & 22 \\
\hline$\overline{\bar{I}}$ & 31 & 19 & 24 & 3 & 17 & 7 & 11 & 12 & 13 & 16 & 24 & 5 & 3 & 10 & $\overline{6}$ & 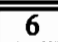 & 22 & 17 & $\bar{~} \overline{77}$ & 7 & 16 & 25 \\
\hline 2 & 19 & 31 & 18 & 1 & 12 & 3 & 9 & 8 & 7 & 10 & 15 & 3 & 3 & 7 & 6 & 3 & 18 & 15 & 5 & 3 & 10 & 18 \\
\hline 3 & 24 & 18 & 31 & 3 & 15 & 6 & 9 & 12 & 11 & 14 & 21 & 4 & 3 & 10 & 6 & 6 & 20 & 17 & 7 & 5 & 14 & 21 \\
\hline 4 & 3 & 1 & 3 & 31 & 2 & 2 & 0 & 1 & 3 & 3 & 1 & 1 & 0 & 0 & 3 & 2 & 2 & 1 & 2 & 1 & 2 & 3 \\
\hline 5 & 17 & 12 & 15 & 2 & 31 & 4 & 6 & 6 & 9 & 11 & 11 & 3 & 2 & $\overline{5}$ & 5 & 4 & 11 & 9 & 5 & 5 & 9 & 14 \\
\hline 6 & 7 & 3 & 6 & 2 & 4 & 31 & 3 & 1 & 6 & 4 & 5 & 0 & 1 & 1 & 2 & 2 & 4 & 2 & 2 & 3 & 5 & 6 \\
\hline 7 & 11 & 9 & $\overline{9}$ & $\overline{0}$ & 6 & 3 & 31 & 6 & 5 & 7 & 9 & $\overline{0}$ & 2 & 4 & 3 & 1 & 9 & 5 & 1 & 4 & 8 & 8 \\
\hline 8 & 12 & 8 & 12 & 1 & 6 & 1 & 6 & 31 & 0 & 3 & 11 & 2 & 1 & 8 & 2 & 4 & 10 & 6 & 5 & 1 & 7 & 8 \\
\hline 9 & 13 & 7 & 11 & 3 & 9 & 6 & 5 & 0 & 31 & 12 & 8 & 1 & 2 & 2 & 5 & 3 & 8 & 5 & 2 & 5 & 9 & 11 \\
\hline 10 & 16 & 10 & 14 & 3 & 11 & 4 & 7 & 3 & 12 & 31 & 10 & 3 & 2 & 2 & 6 & 3 & 11 & 8 & 2 & 5 & 11 & 13 \\
\hline 11 & 24 & 15 & 21 & 1 & 11 & 5 & 9 & 11 & 8 & 10 & 31 & 4 & 3 & 11 & 2 & 5 & 21 & 18 & 6 & 4 & 12 & 20 \\
\hline 12 & 5 & 3 & 4 & 1 & 3 & 0 & 0 & 2 & $\overline{1}$ & 3 & 4 & 31 & 1 & 2 & 2 & 1 & 4 & 5 & 3 & 0 & 3 & 4 \\
\hline 13 & 3 & 3 & 3 & 0 & 2 & 1 & 2 & 1 & 2 & 2 & 3 & 1 & 31 & $\overline{1}$ & 2 & $\overline{1}$ & 3 & 2 & 1 & 1 & 3 & 3 \\
\hline 14 & 10 & 7 & 10 & $\overline{0}$ & 5 & 1 & 4 & 8 & 2 & 2 & 11 & 2 & 1 & 31 & 0 & 3 & 9 & 7 & 5 & 1 & 5 & 8 \\
\hline 15 & 6 & 6 & 6 & 3 & 5 & 2 & 3 & 2 & 5 & 6 & 2 & 2 & 2 & 0 & 31 & 1 & 5 & 3 & 3 & 2 & 5 & 6 \\
\hline 16 & 6 & 3 & 6 & 2 & 4 & 2 & 1 & 4 & 3 & 3 & 5 & 1 & 1 & 3 & 1 & 31 & 4 & 2 & 4 & 1 & 2 & 4 \\
\hline 17 & 22 & 18 & 20 & 2 & 11 & 4 & 9 & 10 & 8 & 11 & 21 & 4 & 3 & 9 & 5 & 4 & 31 & 17 & 5 & 3 & 12 & 19 \\
\hline 18 & 17 & 15 & 17 & 1 & 9 & 2 & 5 & 6 & 5 & 8 & 18 & 5 & 2 & 7 & 3 & 2 & 17 & 31 & 4 & 3 & 7 & 17 \\
\hline 19 & 7 & 5 & 7 & 2 & 5 & 2 & 1 & 5 & 2 & 2 & 6 & 3 & 1 & 5 & 3 & 4 & 5 & 4 & 31 & 1 & 3 & 6 \\
\hline 20 & 7 & 3 & 5 & 1 & 5 & 3 & 4 & 1 & 5 & 5 & 4 & 0 & 1 & 1 & 2 & 1 & 3 & 3 & 1 & 31 & 4 & 6 \\
\hline 21 & 16 & 10 & 14 & 2 & 9 & 5 & 8 & 7 & 9 & 11 & 12 & 3 & 3 & 5 & 5 & 2 & 12 & 7 & 3 & 4 & 31 & 13 \\
\hline 22 & 25 & 18 & 21 & 3 & 14 & 6 & 8 & 8 & 11 & 13 & 20 & 4 & 3 & $\overline{8}$ & 6 & 4 & 19 & 17 & 6 & 6 & 13 & 31 \\
\hline 23 & 27 & 19 & 23 & 2 & 15 & 5 & 10 & 11 & 12 & 15 & 23 & 5 & 3 & 9 & 6 & 4 & 22 & 18 & 6 & 5 & 14 & 22 \\
\hline 24 & 29 & 19 & 25 & 3 & 15 & 6 & 10 & 13 & 12 & 15 & 24 & 5 & 3 & 11 & 6 & 6 & 22 & 18 & 7 & 6 & 15 & 25 \\
\hline 25 & 26 & 18 & 23 & 2 & 13 & 5 & 10 & 12 & 10 & 13 & 24 & 4 & 3 & 11 & 5 & 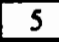 & 22 & 18 & 6 & 4 & 15 & 23 \\
\hline 26 & 30 & 19 & 25 & 3 & 17 & 6 & 11 & 13 & 12 & 16 & 24 & 5 & 3 & 10 & 6 & 6 & 22 & 18 & 7 & 7 & 16 & 25 \\
\hline 27 & 25 & 18 & 22 & 3 & 14 & 6 & 10 & 11 & 11 & 14 & 20 & 5 & 3 & 9 & 6 & 5 & 18 & 15 & 7 & 6 & 14 & 22 \\
\hline 28 & 6 & 4 & 5 & 0 & 2 & 0 & 2 & 4 & 0 & 0 & 6 & 2 & 0 & 4 & 0 & 1 & 5 & 6 & 2 & 0 & 1 & 5 \\
\hline 29 & 24 & 16 & 18 & 2 & 10 & 4 & 8 & 10 & 8 & 11 & 20 & 3 & 1 & 8 & 4 & 4 & 19 & 14 & 5 & 4 & 12 & 20 \\
\hline 30 & 14 & 9 & 14 & 2 & 8 & 4 & 5 & 8 & 7 & 6 & 12 & 3 & 2 & 7 & 4 & 2 & 12 & 10 & 4 & 3 & 9 & 13 \\
\hline 31 & 27 & 18 & 23 & 2 & 14 & 5 & 10 & 12 & 10 & 14 & 23 & 5 & 3 & 10 & 5 & 5 & 21 & 17 & 6 & 5 & 15 & 23 \\
\hline 32 & 28 & 17 & 23 & 3 & 15 & 6 & 10 & 11 & 12 & 15 & 23 & 5 & 3 & 9 & 6 & 5 & 21 & 17 & 6 & 6 & 15 & 23 \\
\hline 33 & 18 & 12 & 16 & 2 & 8 & 3 & 6 & 9 & 6 & 9 & 17 & 4 & 3 & 6 & 4 & 3 & 16 & 14 & 3 & 3 & 10 & 17 \\
\hline 34 & 23 & 16 & 19 & 2 & 13 & 4 & 8 & 11 & 8 & 10 & 19 & 4 & 1 & 10 & 4 & 5 & 18 & 16 & 7 & 5 & 10 & 20 \\
\hline 35 & 12 & 5 & 9 & 1 & 5 & 3 & 5 & 3 & 6 & 8 & 9 & 1 & 2 & 2 & 2 & 1 & 7 & 6 & 0 & 4 & 8 & 10 \\
\hline 36 & 26 & 18 & 24 & 3 & 14 & 5 & 9 & 12 & 11 & 14 & 22 & 5 & 3 & 10 & 6 & 6 & 21 & 17 & 7 & 5 & 14 & 23 \\
\hline 37 & 25 & 17 & 23 & 3 & 14 & 5 & 9 & 11 & 11 & 14 & 21 & 5 & 3 & 9 & 6 & 6 & 20 & 16 & 7 & 5 & 14 & 22 \\
\hline 38 & 15 & 10 & 12 & 1 & 7 & 4 & 9 & 5 & 8 & 11 & 12 & 0 & 2 & 4 & 3 & 2 & 12 & 7 & 1 & 5 & 10 & 13 \\
\hline 39 & 26 & 16 & 22 & 3 & 12 & 6 & 9 & 10 & 11 & 14 & 22 & 5 & 3 & 8 & 5 & 4 & 20 & 19 & 5 & 6 & 14 & 23 \\
\hline 40 & 26 & 16 & 22 & 3 & 14 & 6 & 10 & 11 & 12 & 15 & 22 & 5 & 3 & 9 & 6 & 5 & 20 & 15 & 6 & 6 & 15 & 22 \\
\hline 41 & 24 & 14 & 20 & 3 & 12 & 6 & 9 & 10 & 11 & 14 & 19 & 4 & 3 & 7 & 5 & 4 & 18 & 15 & 4 & 6 & 14 & 21 \\
\hline 42 & 26 & 15 & 22 & 3 & 13 & 6 & 9 & 11 & 11 & 14 & 23 & 5 & 3 & 9 & 5 & 5 & 20 & 17 & 6 & 6 & 14 & 22 \\
\hline 43 & 26 & 18 & 22 & 2 & 14 & 5 & 10 & 12 & 10 & 12 & 22 & 4 & 3 & 10 & 6 & 5 & 20 & 17 & 7 & 6 & 13 & 23 \\
\hline 44 & 17 & 11 & 14 & 1 & 8 & 2 & 7 & 9 & 5 & 8 & 16 & 3 & 3 & 7 & 4 & 3 & 14 & 13 & 4 & 4 & 8 & 13 \\
\hline 45 & 16 & 10 & 15 & 2 & 7 & 4 & 4 & 7 & 5 & 6 & 15 & 4 & 2 & 6 & 3 & 2 & 14 & 13 & 4 & 2 & 9 & 16 \\
\hline
\end{tabular}




\begin{tabular}{|c|c|c|c|c|c|c|c|c|c|c|c|c|c|c|c|c|c|c|c|c|}
\hline 23 & 24 & 25 & 26 & 27 & 28 & 29 & 30 & 31 & 32 & 35 & 36 & 37 & 38 & 39 & 40 & 41 & 42 & 43 & 44 & 45 \\
\hline 27 & $\overline{229}$ & $\overline{266}$ & $\overline{30}$ & 25 & $\overline{6}$ & $\overline{224}$ & 114 & 27 & 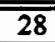 & 112 & $\overline{26}$ & 25 & $\overline{115}$ & 26 & $\overline{\overline{26}}$ & $\overline{224}$ & 26 & 26 & 17 & 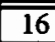 \\
\hline 19 & 19 & 18 & 19 & 18 & 4 & 16 & 9 & 18 & 17 & 5 & 18 & \begin{tabular}{|l|}
17 \\
\end{tabular} & 10 & 16 & 16 & 14 & 15 & 18 & 11 & \begin{tabular}{|l}
10 \\
\end{tabular} \\
\hline 23 & 25 & 23 & 25 & 22 & 5 & 18 & 14 & 23 & 23 & 9 & 24 & 23 & 12 & \begin{tabular}{|l|}
22 \\
\end{tabular} & 22 & 20 & 22 & 22 & 14 & 15 \\
\hline 2 & 3 & 2 & 3 & 3 & 0 & 2 & 2 & 2 & 3 & 1 & 3 & 3 & 1 & 3 & 3 & 3 & 3 & 2 & 1 & 2 \\
\hline 15 & 15 & 13 & 17 & 14 & 2 & 10 & 8 & 14 & 15 & 5 & 14 & 14 & 7 & 12 & 14 & 12 & 13 & 14 & 8 & 7 \\
\hline 5 & 6 & 5 & 6 & 6 & 0 & 4 & 4 & 5 & 6 & 3 & 5 & 5 & 4 & 6 & 6 & 6 & 6 & 5 & 2 & 4 \\
\hline 10 & 10 & 10 & 11 & 10 & 2 & 8 & 5 & 10 & 10 & 5 & 9 & 9 & 9 & 9 & 10 & 9 & 9 & 10 & 7 & 4 \\
\hline 11 & 13 & 12 & 13 & 11 & 4 & 10 & 8 & 12 & 11 & 3 & 12 & 11 & 5 & \begin{tabular}{|l|}
10 \\
\end{tabular} & 11 & 10 & 11 & 12 & 9 & 7 \\
\hline 12 & 12 & 10 & 12 & 11 & 0 & 8 & 7 & 10 & 12 & 6 & 11 & 11 & 8 & \begin{tabular}{|l|}
11 \\
\end{tabular} & 12 & 11 & 11 & 10 & 5 & 5 \\
\hline 15 & 15 & 13 & 16 & 14 & 0 & 11 & 6 & 14 & 15 & 8 & 14 & 14 & 11 & \begin{tabular}{|l|}
14 \\
\end{tabular} & 15 & 14 & 14 & 12 & 8 & 6 \\
\hline 23 & 24 & 24 & 24 & 20 & 6 & 20 & 12 & 23 & 23 & 9 & 22 & 21 & 12 & \begin{tabular}{|l|}
22 \\
\end{tabular} & 22 & 19 & 23 & 22 & 16 & 15 \\
\hline 5 & 5 & 4 & 5 & 5 & 2 & 3 & 3 & 5 & 5 & 1 & 5 & 5 & 0 & 5 & 5 & 4 & 5 & 4 & 3 & 4 \\
\hline 3 & $\overline{3}$ & 3 & 3 & 3 & 0 & 1 & 2 & 3 & 3 & 2 & 3 & 3 & $\overline{2}$ & 3 & 3 & 3 & 3 & 3 & 3 & 2 \\
\hline$\overline{9}$ & 11 & 11 & 10 & 9 & 4 & 8 & 7 & 10 & 9 & 2 & 10 & 9 & 4 & $\overline{8}$ & 9 & 7 & 9 & 10 & 7 & 6 \\
\hline 6 & 6 & 5 & 6 & 6 & 0 & 4 & 4 & 5 & 6 & 2 & 6 & 6 & 3 & 5 & 6 & 5 & 5 & 6 & 4 & 3 \\
\hline 4 & 6 & 5 & 6 & 5 & 1 & 4 & 2 & 5 & 5 & 1 & 6 & 6 & 2 & 4 & 5 & 4 & 5 & 5 & 3 & 2 \\
\hline 22 & 22 & 22 & 22 & 18 & 5 & 19 & 12 & 21 & 21 & 7 & 21 & 20 & 12 & 20 & 20 & 18 & 20 & 20 & 14 & 14 \\
\hline 18 & 18 & 18 & 18 & 15 & 6 & 14 & 10 & 17 & 17 & 6 & 17 & 16 & 7 & \begin{tabular}{|l|}
19 \\
\end{tabular} & 15 & 15 & 17 & 17 & 13 & 13 \\
\hline 6 & 7 & 6 & 7 & 7 & 2 & 5 & 4 & 6 & 6 & 0 & 7 & 7 & 1 & 5 & 6 & 4 & 6 & 7 & 4 & 4 \\
\hline 5 & 6 & 4 & 7 & 6 & 0 & 4 & 3 & 5 & 6 & 4 & 5 & $\overline{5}$ & 5 & $\overline{6}$ & 6 & 6 & 6 & 6 & 4 & 2 \\
\hline 14 & 15 & 15 & 16 & 14 & 1 & 12 & 9 & 15 & 15 & 8 & 14 & 14 & 10 & 14 & 15 & 14 & 14 & 13 & 8 & 9 \\
\hline 22 & 25 & 23 & 25 & 22 & 5 & 20 & 13 & 23 & 23 & 10 & 23 & 22 & 13 & 23 & 22 & 21 & 22 & 23 & 13 & 16 \\
\hline 31 & 26 & 24 & 26 & 22 & 5 & 21 & 15 & 24 & 25 & 9 & 24 & 23 & 13 & 24 & 24 & 22 & 24 & 24 & 16 & 15 \\
\hline 26 & 31 & 26 & 29 & 25 & 6 & 23 & 15 & 27 & 27 & 11 & 27 & 25 & 14 & 26 & 26 & 24 & 26 & 26 & 17 & 16 \\
\hline 24 & 26 & 31 & 26 & 21 & 6 & 22 & 14 & 25 & 24 & 10 & 24 & 23 & 14 & \begin{tabular}{|l|}
23 \\
\end{tabular} & 23 & 21 & 23 & 24 & 16 & 16 \\
\hline 26 & 29 & 26 & 31 & 25 & 6 & 23 & 14 & 27 & 28 & 12 & 27 & 26 & 15 & 26 & 26 & 24 & 26 & 26 & 17 & 16 \\
\hline 22 & 25 & 21 & 25 & 31 & 5 & 19 & 12 & 23 & 23 & 9 & 23 & 22 & 13 & 22 & 23 & 20 & 22 & 22 & 14 & 14 \\
\hline 5 & 6 & $\overline{6}$ & 6 & 5 & 31 & 5 & 3 & 6 & 5 & 2 & 6 & $\overline{6}$ & 1 & $\overline{6}$ & $\overline{4}$ & 4 & 5 & $\overline{6}$ & 4 & 5 \\
\hline 21 & 23 & 22 & 23 & 19 & 5 & 31 & 10 & 22 & 21 & 8 & 21 & 20 & 13 & 20 & 20 & 18 & 20 & 21 & 13 & 13 \\
\hline 15 & 15 & 14 & 14 & 12 & 3 & 10 & 31 & 13 & 14 & 4 & 14 & 13 & $\overline{6}$ & \begin{tabular}{|l|}
14 \\
\end{tabular} & 14 & 14 & 14 & 14 & 8 & 11 \\
\hline 24 & 27 & 25 & 27 & 23 & 6 & 22 & 13 & 31 & 25 & 11 & 25 & 24 & 14 & 24 & 24 & 22 & 24 & 24 & 16 & 16 \\
\hline 25 & 27 & 24 & 28 & 23 & 5 & 21 & 14 & 25 & 31 & 11 & 25 & 24 & 14 & \begin{tabular}{|l|}
25 \\
\end{tabular} & 25 & 23 & 25 & 24 & 16 & 16 \\
\hline 17 & 19 & 18 & 19 & 17 & 4 & 15 & 11 & 18 & 18 & 8 & 18 & 17 & 10 & 18 & 18 & 17 & 18 & 17 & 13 & 14 \\
\hline 21 & 23 & 21 & 23 & 19 & 6 & 19 & 12 & 21 & 21 & 6 & 21 & 20 & 11 & 20 & 20 & 18 & 20 & 22 & 14 & 13 \\
\hline 9 & 11 & 10 & 12 & 9 & 2 & 8 & 4 & 11 & 11 & 31 & 10 & 10 & 7 & 12 & 10 & 11 & 11 & 9 & 8 & 7 \\
\hline 24 & 27 & 24 & 27 & 23 & 6 & 21 & 14 & 25 & 25 & 10 & 31 & 25 & 13 & 24 & 24 & 22 & 24 & 24 & 15 & 16 \\
\hline 23 & 25 & 23 & 26 & 22 & 6 & 20 & 13 & 24 & 24 & 10 & 25 & 31 & 13 & 23 & 23 & 21 & 23 & 23 & 14 & 16 \\
\hline 13 & 14 & 14 & 15 & 13 & 1 & 13 & 6 & 14 & 14 & 7 & 13 & 13 & 31 & \begin{tabular}{|l|}
13 \\
\end{tabular} & 14 & 13 & 13 & 13 & 9 & 8 \\
\hline 24 & 26 & 23 & 26 & 22 & 6 & 20 & 14 & 24 & 25 & 12 & 24 & 23 & 13 & 31 & 23 & 24 & 25 & 23 & 16 & 17 \\
\hline 24 & 26 & 23 & 26 & 23 & 4 & 20 & 14 & 24 & 25 & 10 & 2 & 23 & 14 & 23 & 31 & 22 & 24 & 23 & 15 & 15 \\
\hline 22 & 24 & 21 & 24 & 20 & 4 & 18 & 14 & 22 & 23 & 11 & 22 & 21 & 13 & 24 & 22 & 31 & 23 & 21 & 14 & 15 \\
\hline 24 & 26 & 23 & 26 & 22 & 5 & 20 & 14 & 24 & 25 & 11 & 24 & 23 & 13 & 25 & 24 & 23 & 31 & 23 & 16 & 16 \\
\hline 24 & 26 & 24 & 26 & 22 & 6 & 21 & 14 & 24 & 24 & 9 & 24 & 23 & 13 & 23 & 23 & 21 & 23 & 31 & 16 & 15 \\
\hline 16 & 17 & 16 & 17 & 14 & 4 & 13 & 8 & 16 & 16 & $\overline{8}$ & 15 & 14 & $\overline{9}$ & 16 & 15 & 14 & 16 & 16 & 31 & 10 \\
\hline 15 & 16 & 16 & 16 & 14 & 5 & 13 & 11 & 16 & 16 & 7 & 16 & 16 & $\overline{8}$ & 17 & 15 & 15 & 16 & 15 & 10 & 31 \\
\hline
\end{tabular}




\begin{tabular}{|c|c|c|c|c|c|c|c|c|c|c|c|c|c|c|c|c|c|c|c|c|c|c|}
\hline & $I$ & 2 & 3 & 4 & 5 & 6 & 7 & 8 & 9 & 10 & 11 & 12 & 13 & 14 & 15 & 16 & 17 & 18 & 19 & 20 & 21 & 22 \\
\hline 1 & 116 & 18 & 22 & 2 & 14 & 5 & 9 & 11 & 10 & 13 & 22 & 3 & 2 & 9 & - & 5 & 21 & 16 & 6 & 5 & 13 & 23 \\
\hline 2 & 18 & 116 & 37 & 10 & 30 & 8 & 12 & 19 & 14 & 19 & 36 & 9 & 13 & 22 & 10 & 8 & 33 & 34 & 10 & 10 & 16 & 35 \\
\hline 3 & 22 & 37 & 116 & 9 & 31 & 11 & 14 & 25 & 18 & 22 & 42 & 13 & 14 & 28 & 14 & 13 & 35 & 33 & 14 & 11 & 23 & 33 \\
\hline 1 & 2 & 10 & 9 & 116 & 25 & 6 & 10 & 13 & 9 & 15 & 6 & 31 & 8 & 13 & 8 & 19 & 1 & 5 & 36 & 6 & 8 & 23 \\
\hline 5 & 14 & 30 & 31 & 25 & 116 & 15 & 20 & 20 & 22 & 29 & 29 & 23 & 15 & 23 & 20 & 16 & 18 & 21 & 25 & 17 & 26 & 40 \\
\hline 6 & 5 & 8 & 11 & 6 & 15 & 116 & 25 & 4 & 36 & 34 & 11 & 5 & 9 & 5 & 26 & 15 & 6 & 3 & 4 & 32 & 25 & 13 \\
\hline 7 & 9 & 12 & 14 & 10 & 20 & 25 & 116 & 9 & 33 & 34 & 15 & 9 & 8 & 8 & 25 & 17 & 11 & 6 & 7 & 28 & 32 & 19 \\
\hline 8 & 11 & 19 & 25 & 13 & 20 & 4 & 9 & 116 & 4 & 11 & 23 & 12 & 15 & 31 & 6 & 8 & 16 & 15 & 13 & 6 & 12 & 25 \\
\hline 9 & 10 & 14 & 18 & 9 & 22 & 36 & 33 & 4 & 116 & 46 & 17 & 8 & 11 & 7 & 36 & 21 & 14 & 8 & 8 & 34 & 38 & 23 \\
\hline 10 & 13 & 19 & 22 & 15 & 29 & 34 & 34 & 11 & 46 & 116 & 21 & 14 & 15 & 9 & 33 & 19 & 17 & 12 & 12 & 36 & 35 & 29 \\
\hline 11 & 22 & 36 & 42 & 6 & 29 & 11 & 15 & 23 & 17 & 21 & 116 & 12 & 14 & 30 & 10 & 10 & 36 & 36 & 11 & 12 & 22 & 35 \\
\hline 12 & 3 & 9 & 13 & 31 & 23 & 5 & 9 & 12 & 8 & 14 & 12 & 116 & 13 & 15 & 9 & 14 & 5 & 6 & 32 & 6 & 11 & 18 \\
\hline 13 & 2 & 13 & 14 & 8 & 15 & 9 & 8 & 15 & 11 & 15 & 14 & 13 & 116 & 16 & 11 & 10 & 9 & 9 & 7 & 13 & 12 & 17 \\
\hline 14 & 9 & 22 & 28 & 13 & 23 & 3 & 8 & 31 & 7 & 9 & 30 & 15 & 16 & 116 & 7 & 9 & 19 & 20 & 15 & 7 & 12 & 30 \\
\hline 15 & 5 & 10 & 14 & 8 & 20 & 26 & 25 & 6 & 36 & 33 & 10 & 9 & 11 & 7 & 116 & 16 & 8 & 4 & 8 & 27 & 28 & 16 \\
\hline 16 & 3 & 8 & 13 & 19 & 16 & 19 & 17 & 8 & 21 & 19 & 10 & 14 & 10 & 9 & 16 & 116 & 4 & 4 & 19 & 14 & 17 & 17 \\
\hline 17 & 21 & 33 & 35 & 1 & 18 & 6 & 11 & 16 & 14 & 17 & 36 & 5 & 9 & 19 & 8 & 4 & 116 & 33 & 3 & 9 & 17 & 29 \\
\hline 18 & 16 & 34 & 33 & 5 & 21 & 3 & 6 & 15 & 8 & 12 & 36 & 6 & 9 & 20 & 4 & 4 & 33 & 116 & 8 & 6 & 11 & 33 \\
\hline 19 & 6 & 10 & 14 & 36 & 25 & 4 & 7 & 13 & 8 & 12 & 11 & 32 & 7 & 15 & 0 & 19 & 3 & 0 & 116 & 5 & 9 & 21 \\
\hline 20 & 5 & 10 & 11 & 6 & 17 & 32 & 28 & 6 & 34 & 36 & 12 & 6 & 13 & 7 & 27 & 14 & 9 & 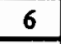 & 5 & 116 & 28 & 15 \\
\hline 21 & 13 & 16 & 23 & 8 & 26 & 25 & 32 & 12 & 38 & 35 & 22 & 11 & 12 & 12 & 28 & 17 & 17 & 11 & 9 & 28 & 116 & 27 \\
\hline 22 & 23 & 35 & 33 & 23 & 40 & 13 & 19 & 25 & 23 & 29 & 35 & 18 & 17 & 30 & 16 & 17 & 29 & 33 & 21 & 15 & 27 & 116 \\
\hline 23 & 24 & 39 & 41 & 24 & 42 & 10 & 18 & 26 & 20 & 25 & 44 & 24 & 16 & 33 & 16 & 14 & 37 & 36 & 26 & 13 & 27 & 49 \\
\hline 24 & 26 & 41 & 43 & 23 & 47 & 14 & 23 & 31 & 25 & 31 & 45 & 21 & 18 & 38 & 19 & 17 & 37 & 39 & 24 & 18 & 29 & 63 \\
\hline 25 & 24 & 42 & 4 & 34 & 49 & 19 & 28 & 31 & 30 & 33 & 45 & 33 & 19 & 38 & 19 & 21 & 38 & 40 & 35 & 18 & 38 & 57 \\
\hline 26 & 27 & 46 & 50 & 35 & 59 & 33 & 40 & 34 & 50 & 53 & 51 & 3 & 24 & 38 & 36 & 32 & 30 & 39 & 34 & 35 & 47 & 66 \\
\hline 27 & 22 & 35 & 39 & 30 & 46 & 37 & 41 & 30 & 51 & 53 & 37 & 26 & 22 & 30 & 37 & 27 & 29 & 26 & 27 & 39 & 45 & 52 \\
\hline 28 & 5 & 17 & 16 & 4 & 13 & 1 & 3 & 9 & 2 & 3 & 18 & 7 & 5 & 13 & 2 & 2 & 16 & 16 & 5 & 3 & 4 & 15 \\
\hline 29 & 22 & 34 & 36 & 17 & 33 & 24 & 26 & 24 & 34 & 35 & 37 & 12 & 15 & 25 & 22 & 17 & 30 & 31 & 15 & 22 & 33 & 42 \\
\hline 30 & 14 & 26 & 29 & 24 & 31 & 12 & 13 & 23 & 18 & 19 & 25 & 20 & 13 & 24 & 11 & 14 & 24 & 25 & 23 & 11 & 21 & 35 \\
\hline 31 & 25 & 46 & 48 & 38 & 57 & 29 & 35 & 34 & 45 & 46 & 48 & 31 & 18 & 38 & 30 & 28 & 40 & 42 & 36 & 29 & 44 & 60 \\
\hline 32 & 20 & 4 & 4 & 37 & 56 & 3 & 36 & 33 & 48 & 48 & 47 & 29 & 19 & 37 & 33 & 28 & 39 & 41 & 34 & 32 & 45 & 59 \\
\hline 33 & 18 & 34 & 34 & 28 & 36 & 18 & 21 & 26 & 26 & 29 & 35 & 2 & 1 & 29 & 10 & 1 & 30 & 35 & 26 & 17 & 25 & 45 \\
\hline 34 & 22 & 34 & 40 & 14 & 28 & 8 & 13 & 29 & 15 & 18 & 39 & 14 & 11 & 32 & 11 & 11 & 35 & 35 & 16 & 10 & 17 & 43 \\
\hline 35 & 10 & 10 & 15 & 19 & 26 & 27 & 28 & 8 & 40 & 37 & 13 & 19 & 12 & 9 & 29 & 19 & 9 & 7 & 18 & 28 & 32 & 24 \\
\hline 36 & 25 & 43 & 49 & 22 & 43 & 27 & 30 & 31 & 37 & 43 & 46 & 22 & 20 & 36 & 26 & 21 & 38 & 39 & 23 & 26 & 35 & 56 \\
\hline 37 & 24 & 38 & 44 & 41 & 56 & 30 & 37 & 31 & 45 & 49 & 42 & 36 & 22 & 38 & 32 & 31 & 33 & 33 & 40 & 31 & 43 & 59 \\
\hline 38 & 14 & 17 & 20 & 14 & 27 & 36 & 37 & 12 & 46 & 46 & 20 & 12 & 14 & 13 & 31 & 21 & 15 & 10 & 11 & 34 & 37 & 25 \\
\hline 39 & 24 & 42 & 44 & 33 & 46 & 24 & 30 & 33 & 37 & 39 & 45 & 32 & 24 & 38 & 25 & 25 & 35 & 38 & 32 & 25 & 39 & 62 \\
\hline 40 & 24 & 34 & 34 & 27 & 43 & 24 & 25 & 22 & 33 & 36 & 35 & 22 & 14 & 28 & 25 & 18 & 27 & 31 & 25 & 21 & 34 & 49 \\
\hline 41 & 22 & 34 & 36 & 26 & 38 & 26 & 27 & 28 & 35 & 38 & 37 & 23 & 18 & 28 & 24 & 21 & 30 & 32 & 24 & 23 & 34 & 52 \\
\hline 42 & 24 & 38 & 41 & 32 & 46 & 24 & 27 & 30 & 39 & 40 & 43 & 26 & 22 & 36 & 27 & 24 & 34 & 39 & 29 & 23 & 35 & 59 \\
\hline 43 & 25 & 46 & 45 & 37 & 51 & 25 & 30 & 35 & 37 & 44 & 46 & 29 & 19 & 39 & 25 & 24 & 39 & 41 & 35 & 25 & 36 & 61 \\
\hline 41 & 16 & 30 & 31 & 18 & 33 & 11 & 13 & 27 & 16 & 21 & 33 & 16 & 14 & 28 & 10 & 9 & 26 & 29 & 18 & 10 & 17 & 42 \\
\hline 45 & 16 & 27 & 31 & 18 & 28 & 13 & 17 & 23 & 21 & 20 & 27 & 15 & 18 & 26 & 14 & 16 & 27 & 27 & 17 & 13 & 20 & 42 \\
\hline
\end{tabular}




\begin{tabular}{|c|c|c|c|c|c|c|c|c|c|c|c|c|c|c|c|c|c|c|c|c|c|c|}
\hline 23 & 24 & 25 & 26 & 27 & 28 & 29 & 30 & 31 & 32 & 33 & 34 & 35 & 36 & 37 & 38 & 39 & 10 & 11 & 42 & 13 & 44 & 45 \\
\hline 24 & 26 & 24 & 27 & 22 & 5 & 22 & 14 & 25 & 26 & 18 & 22 & 10 & 25 & 24 & 14 & 24 & 24 & 22 & 24 & 25 & 16 & 16 \\
\hline 39 & 41 & 42 & 46 & 35 & 17 & 34 & 26 & 46 & 44 & 34 & 34 & 10 & 43 & 38 & 17 & 42 & 34 & 34 & 38 & 46 & 30 & 27 \\
\hline 41 & 43 & 44 & 50 & 39 & 16 & 36 & 29 & 48 & 48 & 34 & 40 & 15 & 49 & 44 & 20 & 44 & 34 & 36 & 41 & 45 & 31 & 31 \\
\hline 24 & 23 & 34 & 35 & 30 & 4 & 17 & 24 & 38 & 37 & 28 & 14 & 19 & 22 & 41 & 14 & 33 & 27 & 26 & 32 & 37 & 18 & 18 \\
\hline 42 & 47 & 49 & 59 & 46 & 13 & 33 & 31 & 57 & 56 & 36 & 28 & 26 & 43 & 56 & 27 & 46 & 43 & 38 & 46 & 51 & 33 & 28 \\
\hline 10 & 14 & 19 & 33 & 37 & 1 & 24 & 12 & 29 & 32 & 18 & 8 & 27 & 27 & 30 & 36 & 24 & 24 & 26 & 24 & 25 & 11 & 13 \\
\hline 18 & 23 & 28 & 40 & 41 & 3 & 26 & 13 & 35 & 36 & 21 & 13 & 28 & 30 & 37 & 37 & 30 & 25 & 27 & 27 & 30 & 13 & 17 \\
\hline 26 & 31 & 31 & 34 & 30 & 9 & 24 & 23 & 34 & 33 & 26 & 29 & 8 & 31 & 31 & 12 & 33 & 22 & 28 & 30 & 35 & 27 & 23 \\
\hline 20 & 25 & 30 & 50 & 51 & 2 & 34 & 18 & 45 & 48 & 26 & 15 & 40 & 37 & 45 & 46 & 37 & 33 & 35 & 39 & 37 & 16 & 21 \\
\hline 25 & 31 & 33 & 53 & 53 & 3 & 35 & 19 & 46 & 48 & 29 & 18 & 37 & 43 & 49 & 46 & 39 & 36 & 38 & 40 & 44 & 21 & 20 \\
\hline 44 & 45 & 45 & 51 & 37 & 18 & 37 & 25 & 48 & 47 & 35 & 39 & 13 & 46 & 42 & 20 & 45 & 35 & 37 & 43 & 46 & 33 & 27 \\
\hline 24 & 21 & 33 & 32 & 26 & 7 & 12 & 20 & 31 & 29 & 23 & 14 & 19 & 22 & 36 & 12 & 32 & 22 & 23 & 26 & 29 & 16 & 15 \\
\hline 16 & 18 & 19 & 24 & 22 & 5 & 15 & 13 & 18 & 19 & 14 & 11 & 12 & 20 & 22 & 14 & 24 & 14 & 18 & 22 & 19 & 14 & 18 \\
\hline 33 & 38 & 38 & 38 & 30 & 13 & 25 & 24 & 38 & 37 & 29 & 32 & 9 & 36 & 38 & 13 & 38 & 28 & 28 & 36 & 39 & 28 & 26 \\
\hline 16 & 19 & 19 & 36 & 37 & 2 & 22 & 11 & 30 & 33 & 19 & 11 & 29 & 26 & 32 & 31 & 25 & 25 & 24 & 27 & 25 & 10 & 14 \\
\hline 14 & 17 & 21 & 32 & 27 & 2 & 17 & 14 & 28 & 28 & 19 & 11 & 19 & 21 & 31 & 21 & 25 & 18 & 21 & 24 & 24 & 9 & 16 \\
\hline 37 & 37 & 38 & 39 & 29 & 16 & 30 & 24 & 40 & 39 & 30 & 35 & 9 & 38 & 33 & 15 & 35 & 27 & 30 & 34 & 39 & 26 & 27 \\
\hline 36 & 39 & 40 & 39 & 26 & 16 & 31 & 25 & 42 & 41 & 35 & 35 & 7 & 39 & 33 & 10 & 38 & 31 & 32 & 39 & 41 & 29 & 27 \\
\hline 26 & 24 & 35 & 34 & 27 & 5 & 15 & 23 & 36 & 34 & 26 & 16 & 18 & 23 & 40 & 11 & 32 & 25 & 24 & 29 & 35 & 18 & 17 \\
\hline 13 & 18 & 18 & 35 & 39 & 3 & 22 & 11 & 29 & 32 & 17 & 10 & 28 & 26 & 31 & 34 & 25 & 21 & 23 & 23 & 25 & 10 & 13 \\
\hline 27 & 29 & 38 & 47 & 45 & 4 & 33 & 21 & 44 & 45 & 25 & 17 & 32 & 35 & 43 & 37 & 39 & 34 & 34 & 35 & 36 & 17 & 20 \\
\hline 49 & 63 & 57 & 66 & 52 & 15 & 42 & 35 & 60 & 59 & 45 & 43 & 24 & 56 & 59 & 25 & 62 & 49 & 52 & 59 & 61 & 42 & 42 \\
\hline 116 & 67 & 67 & 70 & 53 & 23 & 43 & 40 & 70 & 69 & 47 & 48 & 24 & 60 & 67 & 24 & 68 & 54 & 52 & 66 & 69 & 43 & 45 \\
\hline 67 & 116 & 68 & 77 & 57 & 22 & 49 & 38 & 72 & 72 & 51 & 51 & 27 & 67 & 69 & 29 & 72 & 56 & 57 & 69 & 71 & 47 & 48 \\
\hline 67 & 68 & 116 & 88 & 66 & 23 & 58 & 51 & 92 & 88 & 65 & 52 & 39 & 75 & 86 & 41 & 84 & 70 & 66 & 78 & 88 & 52 & 51 \\
\hline 70 & 77 & 88 & 116 & 86 & 23 & 67 & 54 & 104 & 107 & 72 & 57 & s5 & 84 & 97 & 54 & 97 & 79 & 80 & 90 & 99 & 59 & 58 \\
\hline 53 & 57 & 66 & 86 & 116 & 12 & 64 & 44 & 83 & 84 & 61 & 42 & S5 & 71 & 84 & 60 & 74 & 66 & 68 & 73 & 78 & 47 & 45 \\
\hline 23 & 22 & 23 & 23 & 12 & 116 & 12 & 15 & 23 & 22 & 17 & 22 & 4 & 25 & 18 & 4 & 23 & 13 & 15 & 18 & 23 & 18 & 18 \\
\hline 43 & 49 & 58 & 67 & 64 & 12 & 116 & 39 & 71 & 69 & 54 & 43 & 37 & 67 & 67 & 46 & 61 & 56 & 60 & 63 & 66 & 42 & 42 \\
\hline 40 & 38 & 51 & 54 & 44 & 15 & 39 & 116 & 59 & 61 & 56 & 38 & 25 & 49 & 54 & 23 & 53 & 47 & 50 & 51 & 58 & 43 & 38 \\
\hline 70 & 72 & 92 & 104 & 83 & 23 & 71 & 59 & 116 & 115 & 83 & 57 & 55 & 84 & 101 & 54 & 92 & 81 & 77 & 94 & 106 & 62 & 59 \\
\hline 69 & 72 & 88 & 107 & 84 & 22 & 69 & 61 & 115 & 116 & 84 & 57 & 57 & 83 & 99 & 55 & 94 & 81 & 79 & 95 & 104 & 62 & 60 \\
\hline 47 & 51 & 65 & 72 & 61 & 17 & 54 & 56 & 83 & 84 & 116 & 44 & 40 & 65 & 69 & 37 & 71 & 63 & 62 & 70 & 75 & 51 & 47 \\
\hline 48 & 51 & 52 & 57 & 42 & 22 & 43 & 38 & 57 & 57 & 44 & 116 & 12 & 56 & 52 & 18 & 55 & 40 & 48 & 53 & 59 & 40 & 39 \\
\hline 24 & 27 & 39 & 55 & 55 & 4 & 37 & 25 & 55 & 57 & 40 & 12 & 116 & 40 & ss & 46 & 46 & 40 & 42 & 46 & 46 & 28 & 27 \\
\hline 60 & 67 & 75 & 84 & 71 & 25 & 67 & 49 & 84 & 83 & 65 & 56 & 40 & 116 & 83 & 48 & 79 & 66 & 69 & 75 & 81 & 52 & 50 \\
\hline 67 & 69 & 86 & 97 & 84 & 18 & 67 & 54 & 101 & 99 & 69 & 52 & 55 & 83 & 116 & 57 & 88 & 75 & 75 & 87 & 95 & 54 & 56 \\
\hline 24 & 29 & 41 & 54 & 60 & 4 & 46 & 23 & 54 & s5 & 37 & 18 & 46 & 48 & 57 & 116 & 46 & 43 & 41 & 45 & 48 & 24 & 27 \\
\hline 68 & 72 & 84 & 97 & 74 & 23 & 61 & 53 & 92 & 94 & 71 & 55 & 46 & 79 & 88 & 46 & 116 & 73 & 78 & 85 & 93 & 59 & 60 \\
\hline 54 & 56 & 70 & 79 & 66 & 13 & 56 & 47 & 81 & 81 & 63 & 40 & 40 & 66 & 75 & 43 & 73 & 116 & 69 & 77 & 79 & 49 & 43 \\
\hline 52 & 57 & 66 & 80 & 68 & 15 & 60 & 50 & 77 & 79 & 62 & 48 & 42 & 69 & 75 & 41 & 78 & 69 & 116 & 75 & 77 & 51 & 47 \\
\hline 66 & 69 & 78 & 90 & 73 & 18 & 63 & 51 & 94 & 95 & 70 & 53 & 46 & 75 & 87 & 45 & 85 & 77 & 75 & 116 & 91 & 57 & 59 \\
\hline 69 & 71 & 88 & 99 & 78 & 23 & 66 & 58 & 106 & 104 & 75 & 59 & 46 & 81 & 95 & 48 & 93 & 79 & 77 & 91 & 116 & 63 & 57 \\
\hline 43 & 47 & 52 & 59 & 47 & 18 & 42 & 43 & 62 & 62 & 51 & 40 & 28 & 52 & 54 & 24 & 59 & 49 & 51 & 57 & 63 & 116 & 39 \\
\hline 45 & 48 & 51 & 58 & 45 & 18 & 42 & 38 & 59 & 60 & 47 & 39 & 27 & 50 & 56 & 27 & 60 & 43 & 47 & 59 & 57 & 39 & 116 \\
\hline
\end{tabular}

